\title{
Dynamik magnetischer Flussröhren in Riesensternen und engen Doppelsternen
}

\author{
Dissertation \\ zur Erlangung des Doktorgrades \\ der Mathematisch-Naturwissenschaftlichen Fakultäten \\ der Georg-August-Universität zu Göttingen
}

\author{
vorgelegt von \\ Volkmar Holzwarth \\ aus Ludwigshafen am Rhein
}

Göttingen 2002 
D7

Referent: Prof. K. Beuermann

Korreferent: Prof. M. Schüssler

Tag der mündlichen Prüfung: 9. Juli 2002 
Still round the corner there may wait A new road or a secret gate; And though I oft have passed them by, A day will come at last when I Shall take the hidden paths that run West of the Moon, East of the Sun. J. R. R. Tolkien

The Lord of the Rings - The Return of the King 



\section{Inhaltsverzeichnis}

$\begin{array}{ll}\text { Zusammenfassung } & 1\end{array}$

1 Einleitung 3

2 Grundlagen 11

2.1 Grundgleichungen . . . . . . . . . . . . . . . . . . . . . 12

2.2 Gleichgewicht magnetischer Flussröhren . . . . . . . . . . . . . . . . . 17

2.3 Ausbruch magnetischer Flussröhren . . . . . . . . . . . . . 21

I Riesensterne $\quad 29$

3 Einleitung und Motivation 31

4 Flussröhren-Dynamik in Riesensternen 35

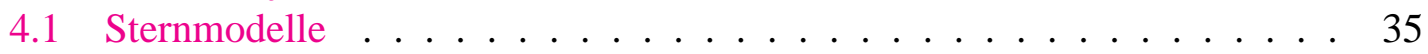

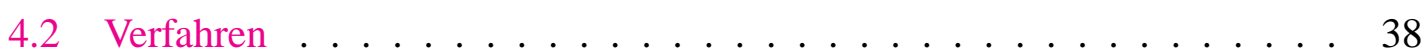

4.3 Einschluss magnetischer Flussröhren . . . . . . . . . . . . . . 42

5 Einschlussmechanismus $\quad 49$

5.1 Kräfteanalyse . . . . . . . . . . . . . . . . . . . 49

5.2 Modell einer polytropen Schichtung . . . . . . . . . . . . . . 51

6 Diskussion $\quad 55$

II Doppelsterne $\quad \mathbf{5 9}$

7 Einleitung und Motivation $\quad 61$

8 Doppelsternmodell $\quad 67$

8.1 Näherung für die Gezeitenwirkung . . . . . . . . . . . . . . . . . . 67

8.2 Referenzsystem . . . . . . . . . . . . . . . . . 74

9 Gleichgewicht und Stabilität $\quad \mathbf{7 5}$

9.1 Stationäres Gleichgewicht _. . . . . . . . . . . . . . . 75

9.2 Lineare Stabilitätsanalyse . . . . . . . . . . . . . . . . . 79 
9.3 Flussröhren in der Äquatorebene . . . . . . . . . . . . . . . . . . . . 82

9.4 Allgemeiner Fall . . . . . . . . . . . . . . . . . . . . . 90

9.5 Diskussion . . . . . . . . . . . . . . . . . . 105

$\begin{array}{ll}10 \text { Simulationen } & 109\end{array}$

10.1 Verfahren . . . . . . . . . . . . . . . . . . 109

10.2 Ergebnisse im Referenzfall . . . . . . . . . . . . . . . . . . 111

10.3 Abhängigkeit von der Umlaufsperiode . . . . . . . . . . . . . . 123

10.4 Diskussion . . . . . . . . . . . . . . . . . . . . . . 124

$\begin{array}{ll}\text { III Strömungsinstabilitåt } & 127\end{array}$

11 Einleitung und Motivation $\quad 129$

12 Horizontale Flussröhren $\quad 133$

12.1 Dispersionsgleichung und Instabilitätskriterium . . . . . . . . . . . . . 133

12.2 Geometrisch-physikalische Deutung . . . . . . . . . . . . . 135

$\begin{array}{ll}13 \text { Toroidale Flussröhren } & 137\end{array}$

13.1 Schnelle Sternrotation . . . . . . . . . . . . . . . . . . . . 137

13.2 Physikalische Interpretation . . . . . . . . . . . . . . . . . 138

13.3 Der Fall der Sonne . . . . . . . . . . . . . . . . . . . . . 139

14 Diskussion $\quad 141$

$\begin{array}{ll}\text { A Koeffizientenmatrizen } & 143\end{array}$

$\begin{array}{ll}\text { Literaturverzeichnis } & \mathbf{1 4 5}\end{array}$

$\begin{array}{ll}\text { Danksagung } & 155\end{array}$

$\begin{array}{ll}\text { Lebenslauf } & 157\end{array}$ 


\section{Abbildungsverzeichnis}

1.1 Fleckenverteilung auf der Sonne . . . . . . . . . . . . . 5

1.2 Ausgedehnte Sonnenfleckengruppe . . . . . . . . . . . . . . . 6

1.3 TRACE-Aufnahmen koronaler Bögen . . . . . . . . . . . . . 7

2.1 Schematische Darstellung des Übergangs einer Flussröhre ins mechanische Kräftegleichgewicht . . . . . . . . . . . . . . . . . . 20

2.2 Alfvénsche Machzahl $M_{\alpha} \ldots \ldots \ldots \ldots \ldots$. . . . . . . . . . . . . . . 21

2.3 Schematische Eigenmodi mit azimutalen Wellenzahlen $m=1 \ldots 5$. . . 23

2.4 Anwachszeiten $\tau$ in einem sonnenähnlichen Stern für $T=15 \mathrm{~d}$. . . . . . 24

2.5 Schwingungsperiode $T_{\text {is }} \ldots \ldots \ldots \ldots \ldots \ldots$

3.1 Grenzlinien im Hertzsprung-Russell Diagramm . . . . . . . . . . . . . . 32

4.1 Vergleich verschiedener Nach-Hauptreihen-Entwicklungssequenzen von Sternen mit Massen $M_{*}=1 \ldots 3 \mathrm{M}_{\odot} \ldots \ldots . \ldots . . \ldots 37$

4.2 Relative Größe des radiativen Kerns im Verlauf der Sternentwicklung . . 37

4.3 Anwachszeit $\tau=100 \mathrm{~d}$ für Flussringe in der Mitte der Overshoot-Region 41

4.4 Beispiele für eine ausbrechende und eine gefangene Flussröhre . . . . . . 43

4.5 Radiale Entwicklung des höchsten Röhrenelements als Funktion der Aus-

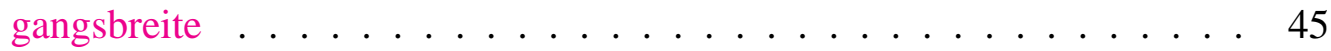

4.6 Änderung des Ausbruchsverhalten magnetischer Flussröhren am Fuße des RGB . . . . . . . . . . . . . . . . . . . . . . 46 46

5.1 Vergleich der Beiträge verschiedener Kräfte zur radialen Beschleunigung des Gipfelelements einer ausbrechenden und einer gefangenen Flussröhre $\quad 50$

5.2 Auslenkung einer Flussröhre im polytropen Schichtungsmodell . . . . . . 52

5.3 Polytrope Schichtung: Verhältnis $\left|a_{\text {out }} / a_{\text {in }}\right|$ am Gipfelelement der halbelliptisch ausgelenkten Flussröhre. . . . . . . . . . . . . . . . . 54

5.4 Nicht-lineare Simulationen im $1 \mathrm{M}_{\odot}$ Modell Nr. 540: Verhältnis $\left|f_{\text {out }} / f_{\text {in }}\right|$ am Gipfelelement aufsteigender Flussröhrenschleifen. . . . . . 54

6.1 Topologie des atmosphärischen Magnetfelds in Riesensternen zu beiden Seiten der CDL . . . . . . . . . . . . . . . . . . 56 56

7.1 Rekonstruierte Oberflächenstrukturen der aktiven Komponente des Systems V711 . . . . . . . . . . . . . . . . . . . . 
8.1 Modell eines Doppelsternsystems ． . . . . . . . . . . . . . . . . 68

8.2 Sternabstand $a$ als Funktion der Umlaufsperiode T (3. Keplersche Gesetz) 69

8.3 Struktur der Teilpotenziale $\Psi_{\text {co }}, \Psi_{\text {rot }}$ und ihrer Summe $\Psi_{\text {tide }} \quad \ldots . . . . \quad 70$

8.4 Relative Massenverteilung $\sigma=M_{*} / M(r)$ in $1 \mathrm{M}_{\odot}$-Sterne unterschiedlicher Entwicklungsstadien . . . . . . . . . . . . . . . . . . 71

9.1 Gleichgewichtskonfiguration im Doppelsternproblem . . . . . . . . . . 76

9.2 Vergleich zwischen der Variation der Dichte $\rho$ und der Superadiabatizität $\delta \quad 78$

9.3 Beispiel für die Struktur instabiler Eigenmodi im Doppelsternproblem . . 82

9.4 Basisfrequenzen binormaler Eigenmodi $\xi_{\mathrm{b}}$ und ihre relative Abweichung aufgrund der Rotationsabplattung . . . . . . . . . . . . . . . . . . . 84

9.5 Relative Frequenzabweichung der Eigenmodi $m=1 \ldots 3$ aufgrund des Doppelsterncharakters . . . . . . . . . . . . 85

9.6 Einhüllende Kurven $\left|\hat{\xi}_{\mathrm{b}}(\phi)\right|$ der Eigenfunktionen $m=1 \ldots 3 \ldots \ldots$. . . . 87

9.7 Variation der Phasengeschwindigkeit $v_{\mathrm{p}}$ der Eigenfunktionen $m=1 \ldots 3 \quad 87$

9.8 Prinzip der Kopplung benachbarter Partialwellen . . . . . . . . . . . . . 89

9.9 Anwachszeiten im Einzelsternproblem unter Berücksichtigung der Rotationsdeformation . . . . . . . . . . . . . . 92

9.10 Relative Änderung der Anwachszeiten aufgrund des Doppelsterncharakters in ursprünglich instabilen Parameterbereichen . . . . . . . . . . . . . 94

9.11 'Instabilitätshintergrund' in ursprünglich stabilen Parameterbereichen aufgrund des Doppelsterncharakters . . . . . . . . . . . . . . . . 94

9.12 Entstehung der Resonanz-Instabilitäten . . . . . . . . . . . . . . . . . . 95

9.13 Eigenvektoren im Einzelsternproblem mit Rotationsabplattung . . . . . . 97

9.14 Azimutwinkel $\phi_{\max }$ der Maxima der radialen Enveloppe $\left|\hat{\xi}_{r}(\phi)\right|$ im Refe-

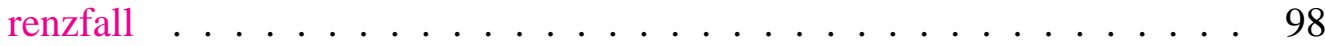

9.15 Minima $\left|\hat{\xi}_{r}\right|_{\text {min }}$ der radialen Enveloppe im Referenzfall . . . . . . . . . . 99

9.16 Radiale Enveloppen $\left|\hat{\xi}_{r}(\phi)\right|$ als Funktion der Länge $\phi$ im Referenzfall . . 101

9.17 Verlauf der relativen Phasengeschwindigkeit $v_{\mathrm{p}} / v_{\mathrm{p}, 0}$ im Referenzfall als

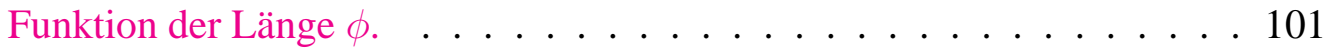

9.18 Anwachszeiten $\tau$ instabiler Eigenmodi als Funktion der Umlaufsdauer T . 102

9.19 Phasenlage $\phi_{\max }$ der Maxima der radialen Enveloppe $\left|\hat{\xi}_{r}\right|$ als Funktion der Umlaufsdauer T . . . . . . . . . . . . . . . . . . . . . . 104

9.20 Minima der radialen Enveloppe $\left|\hat{\xi}_{r}\right|_{\text {min }}$ als Funktion der Umlaufsdauer T. 104

9.21 Schematische Darstellung stabiler und instabiler Flussröhren im Einzelund Doppelsternproblem . . . . . . . . . . . . . . . . . . 106

10.1 Schematische Darstellung der Störung einer toroidalen Flussröhre im Doppelsternproblem . . . . . . . . . . . . . . . 110

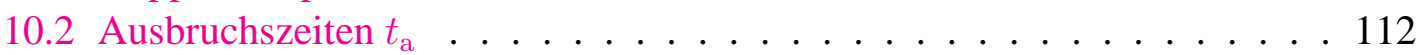

10.3 Polwärtige Ablenkung $\left(\lambda_{\mathrm{a}}-\lambda_{\mathrm{s}}\right)$ als Funktion der Startbreite $\lambda_{\mathrm{s}}$ für verschiedene Feldstärken $B_{\mathrm{s}} \ldots \ldots \ldots$. . . . . . . . . . 113

10.4 Beispiele für Längenverteilungen im Doppelsternproblem . . . . . . . . . 115

10.5 Längenverteilungen für mittlere Startbreiten $\lambda_{\mathrm{s}}=30 \ldots 60^{\circ} \ldots \ldots$. . . . . 116

10.6 Längenverteilungen für hohe Startbreiten $\lambda_{\mathrm{s}}=65 \ldots 75^{\circ} \ldots \ldots$. . . . 118 
10.7 Beispiele für Aufstiegstrajektorien regulärer Fälle und irreguläre Sonderfälle . . . . . . . . . . . . . . . . . . . 119

10.8 Oberflächenverteilungen für Ausgangsfeldstärken $B_{\mathrm{s}}=8 \ldots 13 \cdot 10^{4} \mathrm{G} \cdot 121$

10.9 Oberflächenverteilungen für Ausgangsfeldstärken $B_{\mathrm{s}}=14 \ldots 20 \cdot 10^{4} \mathrm{G} \cdot 122$

10.10Längenverteilungen für mittlere Startbreiten bei Umlaufsdauern $T=$

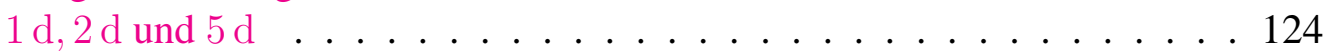

11.1 Widerstandsbeiwert $C_{\mathrm{D}}(R e) \ldots \ldots \ldots \ldots$

12.1 Stabilität horizontaler Flussröhren bei Stokesscher Reibung . . . . . . . . 135

12.2 Geschwindigkeits- und Kräfteverhältnisse in einer horizontalen Flussröhre bei Stokesscher Reibung . . . . . . . . . . . . . . . . 136

13.1 Stabilität toroidaler Flussröhren mit und ohne Reibung für $T=2 \mathrm{~d}$. . . 138

13.2 Vergleich zwischen Stabilitätsanalyse und Simulationen . . . . . . . . . . 139

13.3 Stabilität toroidaler Flussröhren mit und ohne Reibung für $T=27 \mathrm{~d}$. . . 140 


\section{Tabellenverzeichnis}

2.1 Gleichungssystem dünner magnetischer Flussröhren. . . . . . . . . . . 18

4.1 Zustandsgrößen von Riesensternen . . . . . . . . . . . . . . . . . . 36

4.2 Lage und Ausdehnung der Overshoot-Region in Riesensternen . . . . . . 39

8.1 Charakteristische Parameter des Referenz-Doppelsternsystems . . . . . . 74

9.1 Charakteristische Größen am Ort des Referenz-Gleichgewichts . . . . . . 78 


\section{Zusammenfassung}

In dieser Arbeit wird das solare "Paradigma" magnetischer Aktivität auf Riesensterne und enge Doppelsterne übertragen. Die äußeren Konvektionszonen dieser Sterne unterscheiden sich bezüglich ihrer Ausdehnung und Struktur deutlich von der konvektiven Hülle der Sonne, und stellen dadurch ein erweitertes Testfeld für die Modellvorstellungen hinsichtlich der Erzeugung, Verstärkung und des Transports von magnetischem Fluss dar, die für den Fall der Sonne entwickelt worden sind. Diese Vorstellungen basieren auf der Betrachtung des Gleichgewichts, der Stabilität und der dynamischen Entwicklung isolierter Magnetfeldbündel, sog. magnetischer Flussröhren, die in ein weitgehend unmagnetisiertes Plasma eingebettet sind. Der Aufstieg von Flussröhren vom Boden der Konvektionszone und ihr Ausbruch an der Sonnenoberfläche führen zur Bildung bipolarer Fleckengruppen und magnetisch aktiver Regionen.

Die Übertragung dieses Modells auf Riesensterne offenbart einen Einschlussmechanismus für magnetische Flussröhren, der im Verlauf der entwicklungsbedingten Expansion der stellaren Hülle einsetzt, wenn der Krümmungsradius eines sich anfänglich am Boden der stellaren Konvektionszone befindlichen Flussrings weniger als etwa $20 \%$ des Sternradius beträgt. Die zunehmende Krümmung des Gipfelsegments einer aufsteigenden Flussröhrenschleife führt dann zur Dominanz der magnetischen Krümmungskraft über den Auftrieb und beendet den Aufstieg noch innerhalb der Konvektionszone. Dieser Einschlussmechanismus ist weitgehend unabhängig von Einzelheiten der Sternschichtung und anderen Parametern wie etwa der Feldstärke oder der Rotationsrate. Der Einschluss magnetischer Flussröhren führt zum Verschwinden ausgedehnter Aktivitätsgebiete und großskaliger Koronabögen, wodurch die Topologie des atmosphärischen Magnetfelds von überwiegend geschlossenen zu offenen Strukturen übergeht. Dies bietet einen Erklärungsansatz für den beobachteten gravierenden Rückgang koronaler Röntgenemission und das Einsetzen massiver Winde in Riesensternen, die im Verlauf ihrer Entwicklung die sog. Koronagrenzlinie (coronal dividing line) im Hertzsprung-Russell Diagramm überqueren.

In einem rotierenden Einzelstern sind alle Richtungen senkrecht zur Rotationsachse äquivalent, sodass die Speicherung und Entwicklung magnetischer Flussröhren nicht vom Azimutwinkel abhängt. In Doppelsternen wird diese Axialsymmetrie durch die Gezeitenwirkung des Begleitsterns gebrochen. In engen Systemen mit Umlaufsperioden von einigen Tagen sind die entsprechenden Effekte mittels eines Näherungsverfahrens analytisch beschreibbar. Sie führen zu einer $\pi$-periodischen azimutalen Variation der Sternstruktur, die sich sowohl auf das Gleichgewicht als auch die Stabilitätseigenschaften magnetischer Flussringe auswirkt. Die Form der aus kleinen Störungen resultierenden Eigenschwingungen führt bereits am unteren Rand der Konvektionszone zu Vorzugslängen bei der Bildung aufsteigender Flussröhrenschleifen. Im Laufe ihrer Entwicklung übt der Begleit- 
stern einen besonders nachhaltigen Einfluss auf instabile Flussröhren mit der azimutalen Wellenzahl $m=2$ aus. Dies ist auf die resonante Wechselwirkung der $\pi$-periodischen Gezeiteneffekte mit der kongruenten geometrischen Struktur zweier gegenüberliegender aufsteigender Flussröhrenschleifen zurückzuführen. Da es sich hierbei um einen kumulativen Effekt handelt, der sich während des Aufstiegs bis zur Oberfläche aufsummiert, führt der geringe Einfluss des Begleitsterns zu ausgeprägten Inhomogenitäten in den Oberflächenverteilungen ausbrechender Flussröhren. Besonders bei mittleren Breiten zeigen sich markante Häufungspunkte in den Längenverteilungen, deren Orientierung systematisch von der Ausgangsbreite und -feldstärke der Flussröhre abhängt; offenbar gibt es keine Vorzugslängen, die bezüglich der Richtung des Begleitsterns fixiert sind. Generell führt der Gezeiteneinfluss zur Bildung von Häufungspunkten auf gegenüber liegenden Hemisphären des aktiven Sterns, was im Einklang mit einer Reihe von Beobachtungen steht.

Im Verlauf der numerischen Simulationen wurde eine neue Flussröhren-Instabilität gefunden, die im Rahmen einer linearen Stabilitätsanalyse nicht auftritt und auf den Strömungswiderstand einer Flussröhre zurückgeht: Ist die Flussröhre in eine externe Tangentialströmung eingebettet, so führt eine kleine Störung ihrer Gleichgewichtslage zum Einsetzen der Strömungsinstabilität, falls die Phasengeschwindigkeit der Störungswelle langsamer als die externe Strömungsgeschwindigkeit ist. In Sternen wird dieses Instabilitätskriterium von Flussröhren in einem Feldstärkebereich erfüllt, der größenordnungsmäßig mit dem der bisher bekannten, auftriebsbedingten Instabilität übereinstimmt. Die Anwachszeiten der Strömungsinstabilität sind dabei z.T. so kurz, dass sie bei der Betrachtung der Speicherung magnetischer Flussröhren und ihrer dynamischen Entwicklung berücksichtigt werden muss. 


\section{Kapitel 1}

\section{Einleitung}

Die Sonne, unser Zentralgestirn, ist aus astronomischer Sicht ein eher durchschnittlicher und gewöhnlicher Stern. Was sie gegenüber anderen Sternen auszeichnet, ist ihre Nähe, die es uns ermöglicht, Phänomene auf ihrer Oberfläche mit einer sehr guten räumlichen und zeitlichen Auflösung zu beobachten. Dadurch ist es möglich Theorien für die Vielzahl charakteristischer Eigenschaften der Sonne zu überprüfen; weichen Beobachtungen von Vorhersagen theoretischer Modelle ab, so müssen diese entsprechend verfeinert und gegebenenfalls revidiert werden. Allerdings ist die schlüssige Erklärung einer Beobachtung nicht immer ein Garant für die Richtigkeit einer Theorie. In seiner "Kosmos-Vorlesung" in Berlin 1827/28 machte Alexander von Humboldt über das Phänomen der Sonnenflecken folgende Ausführungen:

Die genügendste [Erklärung] scheint, indem wir zu der Hypothese unsere Zuflucht nehmen, dass der Sonnenkörper von zwei Wolkenschichten umgeben sei, von denen die nächsten der Sonne aschfarben, die entferntere aber hell angenommen werden muss. Denken wir uns nun, dass wahrscheinlich auf der Sonne sich Gasarten oder ähnliche Fluide entwickeln, welche bei Aufströmen die beiden Wolkenschichten trennen, und den dunklen Sonnenkörper sichtbar machen, so wird die Erscheinung der Flecken, in der Projektion in welche wir sie erblicken, vollkommen erklärlich.

Heute wissen wir hingegen, dass die Sonne ein selbstleuchtender Körper ist und Sonnenflecken durch starke Magnetfeldkonzentrationen in der Photosphäre verursacht werden.

Außer den dunklen Flecken können noch viele weitere Aktivitätsphänomene in der Sonnenatmosphäre auf den direkten oder indirekten Einfluss magnetischer Felder zurückgeführt werden, wodurch natürlich die Frage nach deren Ursprung aufgeworfen wird. Die Möglichkeit eines primordialen Magnetfelds als Quelle heutiger Aktivitätsphänomene kann schon aufgrund der zyklischen und großräumigen Polaritätsumkehrungen auf der Zeitskala von wenigen Jahre ausgeschlossen werden. Man geht vielmehr davon aus, dass ein hydro-magnetischer Dynamoprozess das Magnetfeld der Sonne gegen die Wirkung der ohmschen Dissipation aufrechterhält und die regulären Umpolungen im Sonnenzyklus bewirkt. Derzeit gängige Dynamomodelle gehen zurück auf Parker (1955a), Babcock (1961) und Leighton (1964, 1969), die einen zyklischen Umwandlungsprozess zwischen toroidalen und poloidalen Magnetfelder vorschlugen, der durch differentielle Rotation 
und turbulente, konvektive Gasströmungen im äußeren Teil des Sonnenkörpers angetrieben wird. Die genauen räumlichen und zeitlichen Abläufe in der Konvektionszone sind derzeit allerdings noch nicht vollständig bekannt und Gegenstand aktueller Forschung. Um spezifische Mechanismen zu identifizieren sind deshalb möglichst umfangreiche und vielfältige Beobachtungen notwendig, aus denen deren charakteristischen Eigenschaften abgeleitet und mit der Theorie verglichen werden können.

Bodengebundene Beobachtungen und Satelliten-Missionen wie SoHO, TRACE oder Yohkoh, haben weite Spektral- und damit Atmosphärenbereiche erschlossen. Die aus derartigen Beobachtungen gewonnen Erkenntnisse zeigen, dass an der Oberfläche ausbrechende Magnetfelder durch Wechselwirkungen mit dem Plasma in der Photosphäre, Chromosphäre und Korona, vielfältige Erscheinungen der Sonnenaktivität hervorrufen. Da die magnetische Energiedichte in der vergleichsweise dünnen Atmosphäre die thermische überwiegt, werden Struktur und Dynamik weitgehend durch das Magnetfeld dominiert: Die Dissipation magnetischer und mechanischer Energie, die beispielsweise mittels magneto-hydrodynamischer Wellen effektiv aus der Konvektionszone in hohe Atmosphärenschichten transportiert wird, stellt einen entscheidenden Beitrag zur Heizung des koronalen Gases dar. Dessen Einschluss innerhalb großräumiger, geschlossener Magnetfeldbögen ermöglicht die Aufrechterhaltung sehr hoher Temperaturen und erlaubt nur einem kleinen Teil der Atmosphäre als Sonnenwind in die Heliosphäre abzuströmen. Sehr schnell ablaufende Änderungen magnetischer Feldkonfigurationen sind dabei Ursache für koronale Massenauswürfe und Teilchenbeschleunigungen bis auf sehr hohe Energien. Die genauen Mechanismen sind derzeit aber noch nicht vollständig geklärt (z.B. Narain \& Ulmschneider 1990, 1996, und darin enthaltene Zitate). Das grundsätzliche Ziel bleibt somit ein möglichst gutes und umfassendes Verständnis solarer Aktivitätssignaturen, um daraus Rückschlüsse für die zugrundeliegenden Mechanismen im Innern ableiten zu können.

\section{Magnetische Aktivitätsphänomene auf der Sonne}

Die wissenschaftliche Beobachtung der Sonne startete zu Beginn des 17. Jahrhunderts mit der Erfindung des Fernrohres. Erwähnt seien hier nur G. Galilei und Chr. Scheiner, deren Beschreibung dunkler Sonnenflecken die 'göttliche Makellosigkeit' unseres Zentralgestirns beendete. Dank konsequenter Beobachtungen konnten schon sehr früh viele Regeln und Gesetzmäßigkeiten der Fleckenerscheinungen erkannt werden, lange bevor G. E. Hale 1908 mit Hilfe des Zeeman-Effekts das Magnetfeld als deren Ursache nachweisen konnte.

Die in der Photosphäre angesiedelten dunklen Sonnenflecken und ihre zyklische Variation sind vermutlich die bekanntesten Merkmale solarer Aktivität. Sie tauchen in zwei Bändern parallel zum Äquator auf: Zu Beginn eines Zyklus liegen die Ausbruchsbreiten bei etwa $\pm 35^{\circ}$ und wandern dann mit der Zeit Richtung Äquator bis etwa $\pm 5^{\circ}$ (Spörers Gesetz), wodurch das charakteristische Schmetterlings-Diagramm der zeitlichen Fleckenverteilung entsteht (Abb. 1.1, oben). Sie treten überwiegend in Form bipolarer Fleckengruppen auf, bei denen der vorausgehende Fleckenherd (der preceeding oder $p$-Fleck) die entgegengesetzte Polarität des nachfolgenden (following oder $f$-Fleck) besitzt. In aufeinander folgenden Zyklen alterniert die Polarität des $p$-Flecks, wodurch es zum 22-jähri- 


\section{DAILY SUNSPOT AREA AVERAGED OVER INDIVIDUAL SOLAR ROTATIONS}
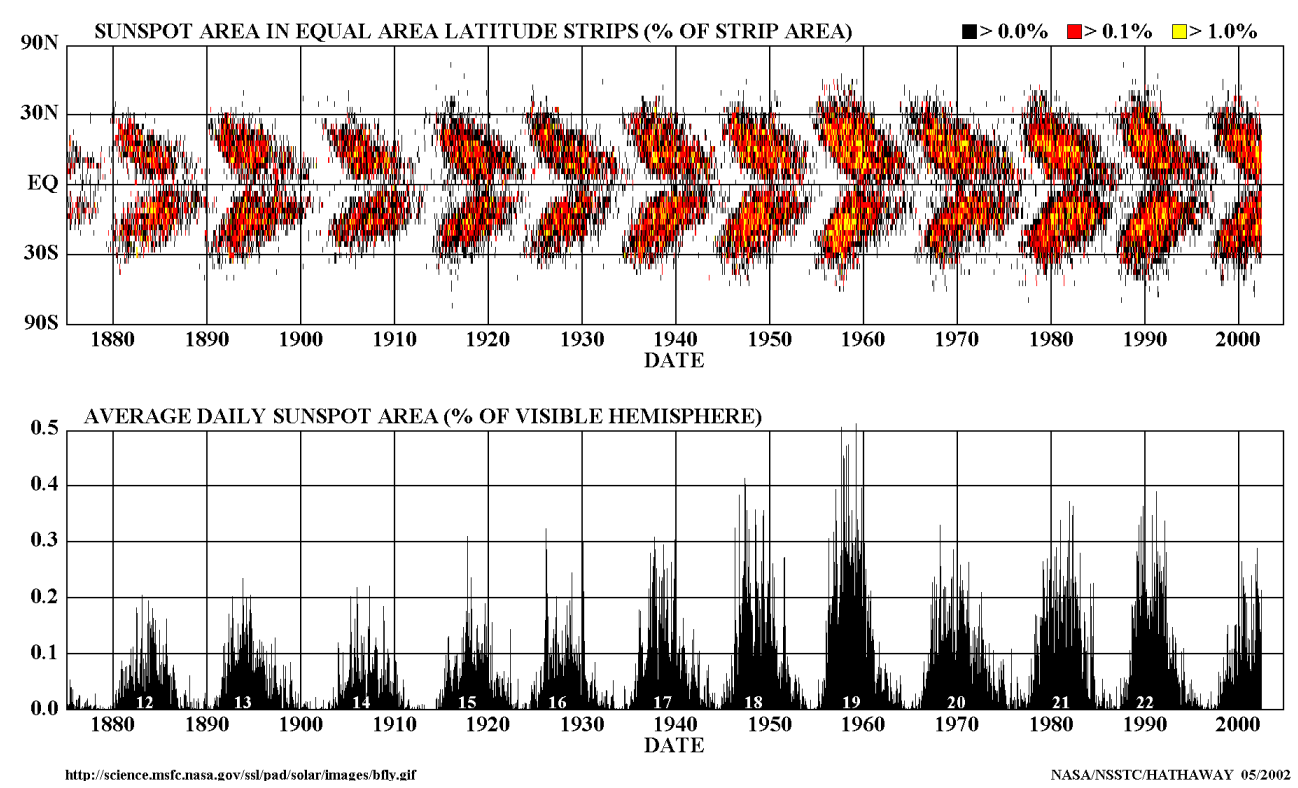

ABBILDUNG 1.1: Oben: Zeitlicher Verlauf der mittleren Fleckenverteilung über mehrere Aktivitätszyklen. Unten: Durchschnittlich bedeckte Fläche der sichtbaren Hemisphäre durch Sonnenflecken. Quelle: D. Hathaway, NASA, Marshall Space Flight Center

gen magnetischen Hale-Zyklus kommt. Dabei sind die Polaritäten der $p$-Flecken auf der nördlichen und südlichen Hemisphäre jeweils entgegengesetzt (Hale-Nicholsonsche Polaritätsregel). Da der vorausgehende Fleck einer bipolaren Gruppe üblicherweise bei etwas kleineren Breiten liegt als der nachfolgende, tritt eine leichte Neigung der Verbindungslinie zwischen $p$ - und $f$-Fleck auf (Joysche Regel); der Neigungswinkel relativ zum Äquator wächst mit der Breite an und beträgt im Mittel etwa $4^{\circ}$. Generell folgen Flecken der differentiellen Rotation der Sonne, wobei in den ersten Tagen nach ihrer Entstehung zusätzlich ein langsames Auseinanderdriften der Komponenten einer bipolaren Gruppe beobachtet wird. Die typische Lebensdauer kleiner Flecken oder Poren sind einige Tage, etwa $90 \%$ aller Flecken sind nach $11 \mathrm{~d}$ verschwunden. Größere Flecken (Abb. 1.2) können sich hingegen teilweise über mehrerer Wochen halten und nach einer Sonnenrotation (von etwa 27 d) nochmals beobachtet werden. Typische Abmessungen einzelner Flecken sind etwa $12000 \mathrm{~km}$ für die Umbra (dunkler Kern) und $28000 \mathrm{~km}$ für die Penumbra (den etwas helleren Hof, vgl. Abb. 1.2). Im Mittel sind weniger als $0.1 \%$ der sichtbaren Sonnenhemisphäre von dunklen Flecken bedeckt, in Zeiten maximaler Aktivität bis zu $0.5 \%$ (Abb. 1.1, unten). Dabei treten oberhalb etwa $50^{\circ}$ Breite keine Flecken auf.

Magnetischer Fluss zeigt die Tendenz in der Nähe bereits vorhandener Flecken auszubrechen, was zur Bildung ausgedehnter und komplexer Aktivitätsregionen führt. Beispielsweise liegen in der Photosphäre gewöhnlich in bzw. nahe bei Aktivitätsgebieten heiße, helle Fackeln, die jedoch meist nur im weißen Licht nahe am Sonnenrand gut zu erkennen sind. Es gibt aber auch polare Fackeln, die nicht mit Flecken korreliert sind. 


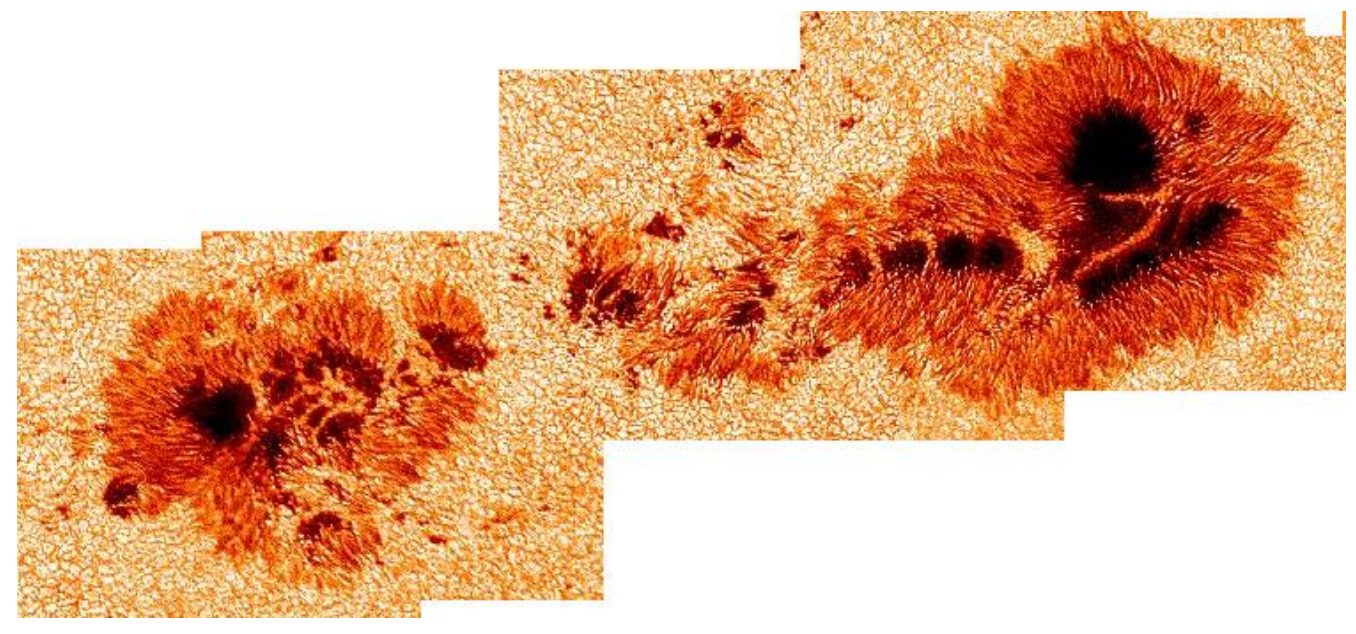

ABBILDUNG 1.2: Ausgedehnte Fleckengruppe in der südlichen Hemisphäre, aufgenommen am 4. September 1998 mit dem Vakuum-Turm-Teleskop in Izaña, Teneriffa. Die kleinsten noch sichtbaren Details sind etwa $350 \mathrm{~km}$ groß, die Gesamtgröße des Gebietes beträgt über $200000 \mathrm{~km}$. Aufnahme: H. Schleicher und H. Wöhl, Kiepenheuer-Institut für Sonnenphysik, Freiburg

Das Magnetfeld in einem Sonnenfleck beträgt $1 \ldots 3 \mathrm{kG}$ und der magnetischer Fluss $10^{20} \ldots 10^{22} \mathrm{Mx}$. Das im räumlichen Mittel sehr schwache Magnetfeld $(<50 \mathrm{G})$ außerhalb Aktivitätsregionen wird dagegen durch eine Vielzahl sehr kleiner $(<100 \mathrm{~km})$ magnetischer Flussröhren gebildet, die sich besonders an den Rändern der Supergranulationszellen ansammeln. Als Reste ursprünglicher Aktivitätsgebiete verteilen sie sich aufgrund differentieller Rotation und meridionaler Zirkulation über die ganze Sonnenoberfläche, bis hinauf zu den Polen.

In der unteren Chromosphäre beobachtet man über Aktivitätsgebieten helle Plages, eine mit photosphärischen Fackeln vergleichbare Erscheinung. Auffällig ist das im Licht der Ca II-Emissionslinien erscheinende chromosphärische Netzwerk, das in etwa die Ränder der Supergranulationszellen nachzeichnet und von magnetischen Flusskonzentrationen hervorgerufen wird.

In der oberen Chromosphäre und Korona füllt das Magnetfeld praktisch den gesamten Raum und bestimmt Struktur und Dynamik dieser Schichten: Großskalige magnetische Flussröhren bilden weite koronale Bögen, die einzelne Sonnenflecken und Aktivitätsgebiete miteinander verbinden (Abb. 1.3). Durch Dissipation elektrischer Ströme und (magneto)hydrodynamischer Wellen wird das Gas innerhalb geschlossener Feldstrukturen auf Temperaturen von $1 \ldots 5 \cdot 10^{6} \mathrm{~K}$ geheizt, wodurch es zu starker, zeitlich und räumlich sehr variabler Emission im EUV und weichen Röntgenbereich kommt ${ }^{1}$. Aufnahmen in diesen Spektralbereichen zeigen teilweise dunkle Gebiete, so genannte koronale Löcher, die insbesondere über den Polen auftreten. Die geringe Strahlungsemission ist hier weniger auf niedrigere Plasmatemperaturen, sondern vielmehr auf deutlich kleinere Teilchenzahldichten zurückzuführen: In diesen Gegenden liegt eine 'offene' Magnetfeldtopologie vor, in der Feldlinien nicht zur Oberfläche zurückführen, sondern weit in die Heliosphäre hin-

\footnotetext{
${ }^{1}$ Die Korona kann auch im sichtbaren Licht hochionisierter Elemente wie Fe X, Fe XIV oder Ca XV gut beobachtet werden.
} 

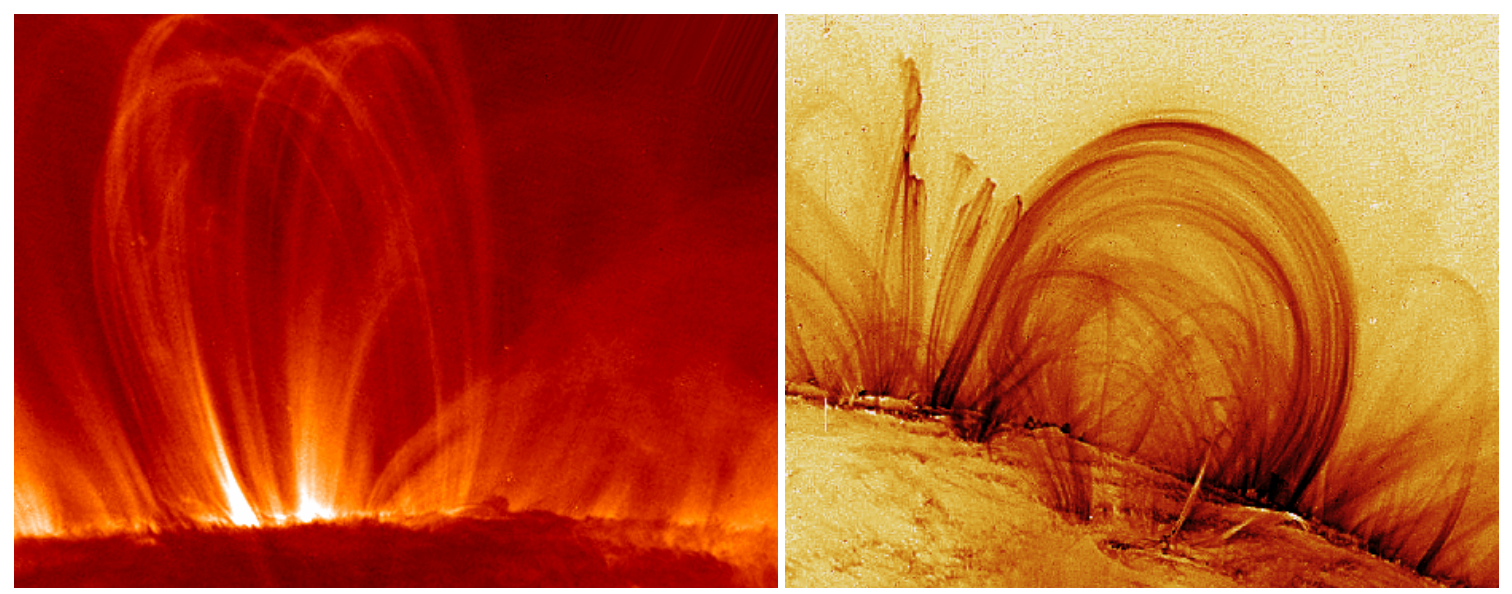

AbBILDUng 1.3: TRACE-Aufnahmen bei $171 \AA$ im EUV, einem Spektralbereich der für Temperatur von etwa $10^{6} \mathrm{~K}$ charakteristisch ist. Die koronalen Bögen erreichen Höhen von näherungsweise $120000 \mathrm{~km}$ (links), andere überspannen einen Bereich der dem 30-fachen Erddurchmesser und mehr entspricht (rechts). TRACE ist eine Mission des Stanford-Lockheed Institute for Space Research und Teil des NASA Small Explorer Programms.

ausreichen und entlang denen Plasma ungehindert von der Oberfläche abströmen und den (schnellen) Sonnenwind bilden kann. Messungen der Helios- und Ulysses-Sonden belegen, dass der Sonnenwind aus polaren koronalen Löchern schneller und weniger dicht ist als der aus äquatorialen Breiten, wo starke magnetische Aktivität und geschlossene Feldlinien vorherrschen.

Korona und Chromosphäre werden im Rahmen vereinfachter Theorien und Modelle oft als zeitlich und räumlich konstant angesehen. Beobachtungen extrem variabler Atmosphärenphänomene stellen diese Annahme jedoch sehr in Frage, zudem teilweise noch gar nicht abzusehen ist, welche weiteren Phänomen sich unterhalb der Auflösungsgrenze abspielen. Auf die Vielzahl kurzzeitiger und explosionsartiger Phänomene wie Radiobursts, koronale Massenauswürfe oder Flares, die sicher magnetischen Ursprungs sind, wird hier jedoch nicht weiter eingegangen. Für umfassendere Erläuterungen zur Sonnenaktivität wird auf gängige Lehr- und Übersichtsliteratur der Astronomie verwiesen (Priest 1982, Stix 1989, Unsöld \& Baschek 1991, Schrijver \& Zwaan 2000, Ossendrijver \& Hoyng 2001, Weiss 2001)

\section{Die solar-stellar connection}

Aufgrund der vergleichsweise geringen Entfernung zu unserem Zentralgestirn werden bei bodengebundenen Beobachtungen mit einem Auflösungsvermögen von weniger als einer Bogensekunde Strukturen von wenigen $100 \mathrm{~km}$ Durchmesser auf der Sonnenoberfläche unterschieden (vgl. Abb. 1.2). Mittels spektroskopischer und polarimetrischer Analyseverfahren können dabei Informationen über lokale Geschwindigkeits- und Magnetfelder gesammelt werden. Darüber hinaus haben in den letzten Jahrzehnten eine Vielzahl von Satelliten praktisch jeden Spektralbereich der Sonne erschlossen und eine zeitlich lückenlose Beobachtung gewährleistet. Sonden, die bis zu einem Abstand von nur 0.3 AU an die 
Sonne heranflogen, konnten gar in situ-Messungen des Sonnenwinds und interplanetaren Magnetfelds vornehmen. Und Mittels der Helioseismologie kann der innere Aufbau der Sonne vermessen und mit den theoretischen Vorhersagen verglichen werden.

So hervorragend die Beobachtungsmöglichkeiten der Sonne auch sein mögen, so haben sie doch den Nachteil, immer nur auf dasselbe Objekt beschränkt zu sein, dessen charakteristische Parameter wie Masse, Radius, Temperatur, chemische Zusammensetzung, Rotationsperiode, Alter etc. vorgegeben sind. Aus der Beobachtung bestimmter Merkmale bei einem Individuum ist es jedoch nicht möglich, auf die Ausprägung vergleichbarer — und möglicherweise charakteristischer - Merkmale einer ganzen Klasse zu schließen oder darauf eine generell gültige Theorie aufzubauen; eine allgemeine Theorie über magnetische Aktivität muss auch auf andere Sterne übertragbar sein und dort vergleichbare Phänomene zutreffend beschreiben.

In den letzten Jahrzehnten wurden Techniken entwickelt, welche die Beobachtung von Aktivitätsphänomenen auch auf weit entfernten Sternen ermöglichen. Im Fall der Sonne findet man beispielsweise mit zunehmender magnetischer Aktivität ein Anwachsen der mittleren chromosphärischen Ca II-Linienkernemission und der koronalen Röntgenstrahlung. Daraus erwuchs ab 1966 die kontinuierliche Erfassung des Mt. Wilson Ca II-S-Index einer Vielzahl von Sternen, der Hinweise über das Ausmaß stellarer chromosphärischer Aktivität sowie über die Existenz und den Verlauf stellarer Aktivitätszyklen gibt (Wilson 1968, Duncan et al. 1991). Auch die Ergebnisse des ROSAT All-Sky Survey (RASS) trugen mit einer immensen Zahl an Röntgenbeobachtungen stellarer Koronen zum Verständnis magnetischer Aktivität bei (z.B. Schmitt 1997). Erwähnenswert sind darüber hinaus verfeinerte Verfahren zur Rekonstruktion stellarer Oberflächenstrukturen wie etwa das Doppler-Imaging (Vogt \& Penrod 1983), Zeeman-Doppler Imaging (Semel 1989, Donati et al. 1997) oder eclipse mapping (Collier Cameron 1997): Zeitserien photometrischer und spektroskopischer Beobachtungen werden kombiniert, um aus der rotations-periodischen Modulation der Lichtkurven und Linienprofile mittels Inversionsverfahren Temperaturinhomogenitäten und Magnetfelder in Sternatmosphären zu bestimmen.

Der Vorteil bei Untersuchungen stellarer Aktivität ist die sehr große Anzahl an Objekten und Klassen ${ }^{2}$, die durch ihre Heterogenität ein optimales Testfeld für Theorien und Modelle darstellen: Ein wichtiger Aspekt der komplementären Beziehung zwischen Sonne und Sternen, der so genannten solar-stellar connection, ist die Prüfung der Übertragbarkeit von Erkenntnissen, die auf Grundlage des Studiums der Sonne gefunden wurden, auf Sterne, deren charakteristische Eigenschaften einen großen Parameterbereich umfassen.

\section{Zu dieser Arbeit}

In der vorliegenden Arbeit wird das Paradigma solarer Aktivitätserscheinungen auf entwickelte Einzelsterne (Riesen und Unterriesen) und Komponenten enger, schnell rotierender Doppelsternsysteme übertragen: Aufgrund der starken Expansion während seiner Nach-Hauptreihen-Entwicklung unterscheidet sich die Konvektionszone eines Riesensterns quantitativ deutlich von der solaren Schichtung, während hingegen in engen

\footnotetext{
${ }^{2}$ In Hinblick auf die magnetische Aktivität sind besonders kühle Sterne vom Spektraltyp G-M von Bedeutung.
} 
Doppelsternen Gezeiteneffekte die Aufhebung der Rotationssymmetrie und damit eine gravierende qualitative Änderung der Sternstruktur bewirken. Ausgehend von der Arbeitshypothese, dass die in diesen Sternklassen beobachteten Aktivitätserscheinungen wie im Fall der Sonne - durch an der Oberfläche ausbrechende Magnetfelder hervorgerufen werden, wird in dieser Abhandlung der Einfluss dieser Unterschiede auf die Dynamik und das Ausbruchsverhalten magnetischer Flussröhren untersucht. Bei der Sonne erklärt das verwendete Modell erfolgreich sowohl die Entstehung als auch charakteristische Eigenschaften bipolarer Fleckengruppen. Es umfasst die Speicherung, Stabilität und Entwicklung magnetischer Flussröhren innerhalb der Konvektionszone bis zu ihrem Ausbruch an der Oberfläche.

In Kap. 2 wird das zugrunde liegende Konzept des solaren Dynamos und das Modell dünner magnetischer Flussröhren vorgestellt sowie die notwendigen Ausgangsgleichungen zusammengefasst. Die daran anschließende Behandlung ist in drei Teile gegliedert.

In Teil I wird die Dynamik magnetischer Flussröhren in kühlen Riesen und Unterriesen während ihrer Nach-Hauptreihen-Entwicklung bis zum Fuße des Riesenastes (RGB, red giant branch) untersucht. Kap. 4 beschreibt die markante Änderung des Ausbruchsverhaltens aufsteigender Flussröhren nahe der so genannten Koronagrenzlinie (CDL, coronal dividing line) im Hertzsprung-Russell Diagramm, an der sich die stellare Röntgenemission signifikant ändert, während in Kap. 5 die Ursache für diese Änderung untersucht wird.

In Teil II wird die Untersuchung der Gezeitenwirkung eines Begleitsterns auf magnetische Flussröhren in Doppelsternen dargestellt. Kap. 8 gibt einen Überblick über das verwendete Doppelsternmodell und die Gezeitenwirkung des Begleitsterns, die im Rahmen einer linearen Näherung beschrieben wird. In Kap. 9 werden charakteristische Eigenschaften magnetischer Flussröhren in Doppelsternen untersucht. Dies umfasst ihr stationäres Gleichgewicht am unteren Rand der Konvektionszone sowie ihr Stabilitätsverhalten bei kleinen Störungen. Kap. 10 umfasst die numerischen (nicht-linearen) Simulationen, welche die Entwicklung ausbrechender Flussröhren bis nahe unter die Oberfläche verfolgen.

In Teil III wird eine neuartige Instabilität eingehender untersucht, die im Rahmen der numerischen Simulationen gefunden wurde und auf den hydrodynamischen Strömungswiderstand einer Flussröhre bei Bewegungen relativ zu ihrer Umgebung zurückzuführen ist. Kap. 12 untersucht den analytisch behandelbaren Fall einer horizontalen Flussröhre in einer plan-parallelen Umgebung, während in Kap. 13 die gewonnenen Erkenntnisse auf toroidale Flussröhren in Sternen angewendet werden. 


\section{Kapitel 2}

\section{Grundlagen}

Magnetfelder spielen bei der Strukturbildung und Aufrechterhaltung sehr hoher Temperaturen in den Chromosphären und Koronen von Sternen eine entscheidende Rolle. Bei diversen Prozessen entstehen im Plasma elektrische Ströme, durch deren Ohmsche Dissipation magnetische Energie in Wärme umgesetzt wird. Dieser Verlust magnetischer Energie wird durch an der Oberfläche ausbrechende Magnetfelder ständig ersetzt (z.B. Zwaan 1978), die innerhalb der Konvektionszone durch einen Dynamomechanismus erzeugt und verstärkt wurden. In der Photosphäre tritt magnetischer Fluss hauptsächlich in Form konzentrierter Magnetfeldstrukturen (mit $B \sim 1 \ldots 3 \mathrm{kG}$ ) in Erscheinung, die in ein erheblich schwächer magnetisiertes Plasma (mit $B \lesssim 50 \mathrm{G}$, Stenflo 1989) eingebettet sind. Diese Feldlinien-Bündel, deren Durchmesser um einige Größenordnungen variieren und die ansatzweise durch isolierte magnetische Flussröhren beschrieben werden, haben ihren Ursprung tief im Innern der Konvektionszone, wobei man davon ausgeht, dass sie bis zu deren unteren Rand hinabreichen (Galloway \& Weiss 1981, Moreno-Insertis 1986).

A priori müssen für stellare Magnetfelder verschiedene Verstärkungsmechanismen in Betracht gezogen werden, die in der Konvektionszone auf unterschiedlichen Längen- und Zeitskalen ablaufen: Großskalige Prozesse, die das globale, zyklische Aktivitätsverhalten bestimmen sowie kleinskalige, turbulente Prozesse, die das lokale Geschehen dominieren (Magnetokonvektion). Erklärungsansätze für das großskalige, alternierende Magnetfeld basieren auf einem Dynamomechanismus, der von Plasmaströmungen angetrieben und durch gemittelte Felder mit überlagerten, stochastisch verteilten Fluktuationen beschrieben wird: Die differentielle Rotation erzeugt dabei aus der poloidalen Magnetfeldkomponente die toroidale ( $\Omega$-Effekt), und die Helizität aufsteigender Konvektionszellen, die dieses Feld mit sich nehmen, im Gegenzug wieder die poloidale Komponente ( $\alpha$-Effekt). Ein grundsätzliches Problem ist die längerfristige Speicherung des Magnetfelds, das in der Konvektionszone einem magnetischen Auftrieb unterliegt (Parker 1955b). Neuere Untersuchungen favorisieren einen Verstärkungsmechanismus, der überwiegend im unteren Teil der Konvektionszone arbeitet (z.B. Parker 1993), wobei das Magnetfeld in einem dünnen, stabil geschichteten Bereich am Übergang zum radiativen Kern - der Overshoot-Region - gespeichert wird. Symmetriebetrachtungen und das Auftreten überwiegend in Ost-West-Richtung angeordneter, bipolarer Fleckengruppen lassen auf eine Feldanordnung parallel zum Äquator schließen. Zusammen mit den aus solaren und stellaren Aktivitätszyklen gewonnenen Erkenntnissen über die Zeitskalen möglicher Verstärkungs- 
mechanismen ergibt sich daraus ein Szenario, das typische Aktivitätserscheinungen auf aus der Overshoot-Region stammende Flussröhren zurückführt.

\subsection{Grundgleichungen}

\subsubsection{Gleichungssystem der idealen MHD}

Durch die Kombination der hydrodynamischen Kontinuitäts- und Navier-StokesGleichung, der elektrodynamischen Maxwellgleichungen sowie der thermodynamischen Energie- und Zustandsgleichung entsteht unter Zuhilfenahme typischer Zeit- und Längenskalen $\tau$ bzw. $l$ des Problems im Grenzfall nicht-relativistischer Strömungs- und Phasengeschwindigkeiten $v=l / \tau \ll c$ das Gleichungssystem der Magnetohydrodynamik (MHD):

$$
\begin{aligned}
& \frac{d \rho}{d t}=-\rho \nabla \cdot \mathbf{v} \\
& \rho \frac{d \mathbf{v}}{d t}=-\nabla p-\rho \nabla \Psi_{*}+\rho \nu \Delta \mathbf{v} \\
& -\rho(\boldsymbol{\Omega} \times(\boldsymbol{\Omega} \times \mathbf{r}))+2 \rho(\mathbf{v} \times \boldsymbol{\Omega}) \\
& +\frac{1}{4 \pi}(\nabla \times \mathbf{B}) \times \mathbf{B} \\
& \frac{\rho^{\gamma}}{(\gamma-1)} \frac{d}{d t}\left(\frac{p}{\rho^{\gamma}}\right)=\left.\rho T \frac{d S}{d t}\right|_{\text {ext }} \\
& p=\rho \frac{\Re}{\mu} T \\
& \frac{\partial \mathbf{B}}{\partial t}=\nabla \times(\mathbf{v} \times \mathbf{B})+\eta_{\mathrm{m}} \Delta \mathbf{B}
\end{aligned}
$$

Dabei bezeichnet

$$
\frac{d}{d t} \equiv \frac{\partial}{\partial t}+(\mathbf{v} \cdot \nabla)
$$

den substanziellen (Lagrangeschen) Ableitungsoperator. Es ist $\rho$ die Dichte, $p$ der Gasdruck, $T$ die Temperatur und $\mu$ das mittlere Molekulargewicht des Plasmas, $\mathbf{v}$ dessen Geschwindigkeit und $\mathbf{B}$ das Magnetfeld. $\gamma$ ist der Adiabatenexponent, $\nu$ die kinematische Viskosität und $\eta_{\mathrm{m}}$ die magnetische Diffusivität. Für detaillierte Herleitungen wird auf gängige Lehr- und Fachliteratur verwiesen (z.B. Kippenhahn \& Möllenhoff 1975, Priest 1982).

Aus dem Gravitationspotenzial $\Psi_{*}$ des Sterns ergibt sich die radialsymmetrische Schwerebeschleunigung $\mathbf{g}_{*}=-\nabla \Psi_{*}$. Im Hinblick auf die spätere Anwendung erfolgt die Beschreibung der erweiterten Navier-Stokes-Gleichung (2.2) in einem mit der konstanten Winkelgeschwindigkeit $\Omega$ rotierenden Bezugssystem, in dem zusätzlich Coriolisund Zentrifugalkräfte auftreten. Die Zentrifugalbeschleunigung wird mit Hilfe der Identität $\Omega \times(\Omega \times \mathbf{r})=-\nabla|\Omega \times \mathbf{r}|^{2} / 2$ als Gradient des Zentrifugalpotenzials

$$
\Psi_{\mathrm{rot}}=-\frac{1}{2}|\Omega \times \mathbf{r}|^{2}
$$


dargestellt und mit $\Psi_{*}$ zum effektiven Gravitationspotenzial $\Psi_{\text {eff }}=\Psi_{*}+\Psi_{\text {rot }}$ zusammengefasst. Die resultierende effektive Gravitation $\mathbf{g}_{\text {eff }}=-\nabla \Psi_{\text {eff }}$ ist axialsymmetrisch bezüglich der Rotationsachse $\Omega$.

\section{Ideales Plasma}

Das Größenverhältnis zwischen Transport- und Diffusionsterm wird in der Navier-StokesGleichung (2.2) durch die hydrodynamische und in der Induktionsgleichung (2.5) durch die magnetische Reynoldszahl ausgedrückt,

$$
R e=\frac{l v}{\nu} \quad \text { bzw. } \quad R_{\mathrm{m}}=\frac{l v}{\eta_{\mathrm{m}}},
$$

wobei die magnetische Diffusivität $\eta_{\mathrm{m}}=c^{2} /\left(4 \pi \sigma_{\mathrm{L}}\right)$ von der elektrischen Leitfähigkeit $\sigma_{\mathrm{L}}$ des Plasmas abhängt. Im Zusammenhang mit der Entwicklung aufsteigender Flussröhren stellt der Röhrenradius $l \sim 10^{8} \mathrm{~cm}$ eine typische Länge und die radiale Aufstiegsgeschwindigkeit $v \sim 3 \cdot 10^{3} \mathrm{~cm} / \mathrm{s}$ einer instabilen Flussröhrenschleife eine typische Geschwindigkeit dar. In der Mitte der Konvektionszone herrscht unter der Annahme eines vollständig ionisierten Wasserstoffplasmas eine Leitfähigkeit $\sigma_{\mathrm{L}} \sim 10^{16} \mathrm{~s}^{-1}$ und kinematische Viskosität $\nu \sim 10 \mathrm{~cm}^{2} / \mathrm{s}$ (nach Spitzer 1962) und damit

$$
\left.R e \sim 10^{10} \quad \text { und (mit } \eta_{\mathrm{m}} \sim 7 \cdot 10^{3} \mathrm{~cm}^{2} / \mathrm{s}\right) \quad R_{\mathrm{m}} \sim 10^{6} .
$$

Aufgrund kleiner Viskositäten und hoher Leitfähigkeiten sind in stellaren Konvektionszonen die Reynoldszahlen ausreichend groß, um die Annahme eines idealen (reibungsund widerstandsfreien) Plasmas zu rechtfertigen und die Diffusionsterme gegenüber den Transporttermen zu vernachlässigen.

Eine wichtige Konsequenz, die sich aus $\sigma_{\mathrm{L}} \rightarrow \infty$ ergibt, ist das Alfvénsche Konzept eingefrorener Magnetfeldlinien. Der magnetische Fluss $\Phi$ durch eine von der geschlossenen Kurve $C$ begrenzten Fläche $\mathbf{A}$ wird durch das Integral

$$
\Phi=\int_{C} \mathbf{B} \cdot \mathrm{d} \mathbf{A}
$$

bestimmt. In der idealen MHD ist der magnetische Fluss $\Phi$ eine Erhaltungsgröße, welche die Bewegung der Materie mit dem Magnetfeld verbindet: Relativbewegungen des Plasmas in der Richtung senkrecht zu den Feldlinien sind nicht möglich; bei Transversalströmungen wird das im Plasma 'eingefrorene' Magnetfeld mitgeschleppt. Plasmaströmungen in tangentialer Richtung ( $\| \mathrm{B}$ ) sind hingegen grundsätzlich unabhängig vom Magnetfeld.

\section{Walénsche Gleichung}

Sowohl für die analytische als auch numerische Behandlung erweist es sich als vorteilhaft, die Kontinuitätsgleichung (2.1) mit der Induktionsgleichung (2.5, mit $\eta_{\mathrm{m}}=0$ ) zu kombinieren, was zur Walénsche Gleichung

$$
\frac{d}{d t}\left(\frac{\mathbf{B}}{\rho}\right)=\left(\frac{\mathbf{B}}{\rho} \cdot \nabla\right) \mathbf{v}
$$

führt. 


\section{Einfluss der Strahlung}

In der Energiegleichung (2.3) trägt wegen $R e, R_{\mathrm{m}} \gg 1$ die durch Ohmsche Dissipation elektrischer Ströme und (innere) viskose Reibung entstehende Wärme nicht zur Änderung der spezifischen Entropie $S$ eines Massenelements bei. Dessen innere Energie kann sich jedoch aufgrund von Strahlung ändern, deren Flussdichte $\mathbf{F}_{\text {rad }}$ im Sterninnern generell durch den Diffusionsansatz

$$
\mathbf{F}_{\mathrm{rad}}=-\rho c_{p} k_{\mathrm{rad}} \nabla T \quad \text { mit } \quad k_{\mathrm{rad}}=\frac{16 \sigma}{3} \frac{T^{3}}{\kappa c_{p} \rho^{2}}
$$

beschrieben werden kann (Priest 1982), wobei $c_{p}$ die spezifische Wärmekapazität bei konstantem Druck, $k_{\text {rad }}$ die thermische Diffusivität und $\sigma$ die Stefan-Boltzmann-Konstante ist. Die räumliche Variation des Strahlungsflusses innerhalb des Volumens eines Massenelements führt zu einer Nettowärmeänderung

$$
\left.\rho T \frac{d S}{d t}\right|_{\mathrm{rad}}=-\nabla \cdot \mathbf{F}_{\mathrm{rad}}
$$

die - je nach Vorzeichen - einer Heizung oder Kühlung des Plasmas entspricht. Eine Abschätzung der thermischen Relaxationszeit

$$
\tau_{\mathrm{rad}}=\frac{l^{2}}{k_{\mathrm{rad}}}
$$

ergibt für Flussröhren in der Sonne (mit $k_{\text {rad }} \lesssim 10^{7} \mathrm{~cm}^{2} / \mathrm{s}$ innerhalb der Konvektionszone und $\left.l \sim 10^{8} \mathrm{~cm}\right) \tau_{\text {rad }} \sim 10^{9} \mathrm{~s}(\approx 32 \mathrm{yr})$. Ein Vergleich mit dynamischen Entwicklungszeitskalen $\tau_{\text {dyn }}$ ausbrechender sowie Anwachszeiten $\tau_{\text {is }}$ instabiler Flussröhren im toroidalen Gleichgewicht (vgl. Abschn. 2.3.2) liefert

$$
\tau_{\text {dyn }}=\frac{l}{v} \sim 10^{4} \mathrm{~s} \ll \tau_{\text {is }} \sim 10^{7} \mathrm{~s} \quad \ll \tau_{\text {rad }}
$$

und zeigt, dass die Entwicklung magnetischer Flussröhren i.Allg. adiabatisch mit $p \rho^{-\gamma}=$ const. abläuft.

\subsubsection{Näherung für dünne Flussröhren}

Die Näherung für dünne Flussröhren (Spruit 1981) stellt eine wesentliche Vereinfachung sowohl der analytischen als auch numerischen Behandlung der Dynamik magnetischer Strukturen dar und beruht auf folgenden Annahmen:

$\triangleright$ Die Flussröhre wird durch eine isolierte Magnetfeld-Konzentration gebildet, die in ein ansonsten feldfreies Plasma eingebettet ist; der Magnetfeldsprung zwischen dem Innern und der Umgebung wird durch einen Flächenstrom auf der Röhrenoberfläche bewirkt. Das ideal leitende Plasma kann den Magnetfeldbereich nicht verlassen oder in ihn eindringen. 
$\triangleright$ Der Durchmesser $d$ einer Flussröhre ist klein im Vergleich zu allen anderen für die Dynamik relevanten Längenskalen, wie etwa der Druckskalenhöhe $H_{p}$, dem Krümmungsradius $R$ oder der Wellenlänge $\lambda$ einer Störung der Flussröhre,

$$
d \ll H_{p}, R, \lambda, \ldots
$$

$\triangleright$ Alle Größen $f(f \in[p, \rho, \mathbf{B}, \mathbf{v}, \ldots])$ ändern sich auf einer zirkularen Querschnittsfläche wenig und können durch ihren Wert auf der Röhrenachse hinreichend genau beschrieben werden.

Aufbauend auf diesen Bedingungen wird die innere Struktur einer dünnen Flussröhre in einer Taylorreihe um die Röhrenachse entwickelt, wobei aber nur die jeweils niedrigsten Entwicklungsterme - abhängig von der Symmetrieeigenschaft einer Größe ist dies die 0. oder 1. Ordnung - berücksichtigt werden (Roberts \& Webb 1978, Ferriz-Mas et al. 1989); durch Elimination der Terme 1. Ordnung gelangt man letztlich zu einer Darstellung, bei der die Flussröhre nur durch die Werte auf der Röhrenachse beschrieben wird.

Die durch den Verlauf ihrer Achse idealisierte Flussröhre stellt eine eindimensionale Kurve im dreidimensionalen Raum dar und legt die Verwendung des begleitenden Dreibeins (Tangentialvektor t, Hauptnormalenvektor $\mathbf{n}$, Binormalenvektor $\mathbf{b}$ ) als lokales Bezugssystems nahe. Wenn ihre Lage $\mathbf{r}(s, t)$ durch die Bogenlänge $s$ parametrisiert wird, folgt aus dem Hauptsatz der Kurventheorie

$$
\mathbf{t}=\frac{\partial \mathbf{r}}{\partial s} \quad, \quad \mathbf{n}=R \frac{\partial \mathbf{t}}{\partial s} \quad \text { und } \quad \mathbf{b}=\mathbf{t} \times \mathbf{n}
$$

sowie die Frenetschen Ableitungsformeln

$$
\frac{\partial \mathbf{t}}{\partial s}=\frac{\mathbf{n}}{R} \quad, \quad \frac{\partial \mathbf{n}}{\partial s}=-\frac{\mathbf{t}}{R}+\frac{\mathbf{b}}{R_{\mathrm{t}}} \quad \text { und } \quad \frac{\partial \mathbf{b}}{\partial s}=-\frac{\mathbf{n}}{R_{\mathrm{t}}} .
$$

$R$ ist der lokale Krümmungsradius und $R_{\mathrm{t}}$ der lokale Torsionsradius der Flussröhre. In der Frenet-Basis gilt per definitionem $\mathbf{B}=B \mathbf{t}$, wodurch die Lorenzkraft in magnetische Spannungs- und Druckterme der Form

$$
\frac{1}{4 \pi}(\nabla \times \mathbf{B}) \times \mathbf{B}=\mathbf{n} \frac{1}{R} \frac{B^{2}}{4 \pi}+\mathbf{t} \frac{\partial}{\partial s} \frac{B^{2}}{8 \pi}-\nabla \frac{B^{2}}{8 \pi}
$$

aufgespalten werden kann; dabei wurde die Identität

$$
\mathbf{t} \cdot \nabla \equiv \frac{d}{d s}
$$

verwendet.

\section{Wechselwirkung mit der Umgebung}

Ein Materieaustausch zwischen Umgebung und Flussröhre findet aufgrund der 'eingefrorenen' Feldlinien nicht statt, ein Energietransport bei adiabatischen Zustandsänderungen ebensowenig. Die Wechselwirkung mit der Umgebung erfolgt durch Impulsaustausch an 
der Oberfläche der Flussröhre aufgrund des Druckverlaufs $p_{\mathrm{e}}(s)$ in der umgebenden Konvektionszone und ihres Strömungswiderstands bei transversalen Bewegungen.

Wie sich zeigen lässt (z.B. Stix 1989), ändern sich in einer hydrostatisch geschichteten Umgebung sowohl der Gesamtdruck als auch dessen Ableitung an der Röhrenoberfläche beim Übergang von innen nach außen stetig. Unter Berücksichtigung des magnetischen Drucks im Innern der Flussröhre folgt daraus die Druckgleichgewichtsbedingung

$$
p_{\mathrm{e}}=p+\frac{B^{2}}{8 \pi} .
$$

Unter der Annahme einer (im mitrotierenden Bezugssystem) ruhenden, nichtmagnetischen Konvektionszone ohne differentielle Rotation und meridionale Strömungen wird die Entwicklung der Flussröhre durch die Beziehung

$$
\nabla p_{\mathrm{e}}=\nabla\left(p+\frac{B^{2}}{8 \pi}\right)=\rho_{\mathrm{e}} \mathbf{g}_{\mathrm{eff}}=-\rho_{\mathrm{e}} \nabla \Psi_{\mathrm{eff}}
$$

mit dem Verlauf $\rho_{\mathrm{e}}$ der externen Schichtung gekoppelt.

Das ideal leitende Plasma der Umgebung kann nicht in den felderfüllten Bereich eindringen, weshalb es bei Relativbewegungen senkrecht zur Röhrenachse die Flussröhre umströmen muss; in der Näherung für dünne Flussröhren bleibt bei diesem Vorgang die kreisförmige Querschnittsfläche erhalten. Lokal ist die Situation vergleichbar mit der Umströmung eines Zylinders, bei welcher sich durch Verwirbelungen auf der abströmenden Seite Druckdifferenzen entlang des Zylinderumfangs bilden, die einen Impulsübertrag den aerodynamischen Strömungswiderstand — hervorrufen, der üblicherweise durch die Beziehung

$$
\mathbf{f}_{\mathrm{D}}=-C_{\mathrm{D}} \rho_{\mathrm{e}} \frac{d}{2} v_{\perp}^{2} \hat{\mathbf{v}}_{\perp}
$$

beschrieben wird. Dabei ist $v_{\perp}$ der Betrag und $\hat{\mathbf{v}}_{\perp}$ der (normierte) Richtungsvektor der Relativgeschwindigkeit $\mathbf{v}_{\perp}=\left(\mathbf{v}_{\mathrm{i}}-\mathbf{v}_{\mathrm{e}}\right)_{\perp}$ senkrecht zur lokalen Röhrenachse. Die quadratische Abhängigkeit des Strömungswiderstands (2.23) von $v_{\perp}$ ist adäquat für Probleme bei hohen Reynoldszahlen $R e \gg 1$. Für $R e \lesssim 1$ ist hingegen, dem Stokesschen Reibungsansatz entsprechend, eine lineare Abhängigkeit angemessener. $C_{\mathrm{D}}$ ist der Widerstandsbeiwert, der von der Reynoldszahl $R e$ abhängt und empirisch bestimmt wird; für $R e \gg 1$ gilt bei einem umströmten Zylinder $C_{\mathrm{D}} \approx 1$. Wie genauere Betrachtungen zeigen, ist die effektive Viskosität in stellaren Konvektionszonen aufgrund der turbulenten Gasbewegungen größer als die in Gl. (2.8) verwendete innere Viskosität $\nu$, wodurch $R e$ deutlich reduziert wird. Nach Moreno-Insertis (1984) hat dieser Effekt letztlich aber nur einen geringen Einfluss auf die Entwicklung magnetischer Flussröhren, weshalb in der vorliegenden Arbeit $C_{\mathrm{D}}=1$ beibehalten wird. Auf die Wirkung der aerodynamischen Reibung wird in Kap. 14 im Zusammenhang mit ihrem Einfluss auf das Stabilitätsverhalten magnetischer Flussröhren ausführlicher eingegangen.

Die Wechselwirkung mit der Umgebung umfasst bisher nur den Strömungswiderstand der Flussröhre, aber nicht die Dynamik des externen Mediums. Dieser zusätzliche Aspekt wurde oft durch den Gebrauch der 'vergrößerten Trägheit' (enhanced inertia) einer Flussröhre berücksichtigt (z.B. Choudhuri 1990, Cheng 1992, Fan et al. 1994, Moreno-Insertis et al. 1994) und beruht auf der Überlegung, dass durch die Bewegung 
der Flussröhre auch das externe Medium in Bewegung versetzt und die effektive Trägheit der Flussröhre dadurch erhöht wird. Die konsequente theoretische Beschreibung dieses Effekts ist bis jetzt allerdings strittig und nicht abschließend geklärt; eine umfassende Diskussion findet man bei Moreno-Insertis et al. (1996). Da dieser Effekt im Vergleich mit den anderen Kräften nur klein ist, wird er in dieser Arbeit nicht berücksichtigt (vgl. Caligari 1995).

\section{Gleichungssystem für dünne magnetische Flussröhren}

In Tab. 2.1 sind alle für die (adiabatische) Entwicklung dünner Flussröhren relevanten Gleichungen zusammengefasst. Dabei gilt in der Frenet-Basis (2.17) des begleitenden Dreibeins $\mathbf{B}=B \mathbf{t}$ und $\mathbf{v}=v_{\mathrm{t}} \mathbf{t}+v_{\mathrm{n}} \mathbf{n}+v_{\mathrm{b}} \mathbf{b}$. Der Index 'e' kennzeichnet Größen der umgebenden Sternschichtung am Ort der Flussröhre, interne Größen haben — sofern keine Verwechslungsgefahr besteht — keinen Index.

\subsection{Gleichgewicht magnetischer Flussröhren}

Da die differentielle Rotation im Fall der Sonne keinen signifikanten Einfluss auf das Aufstiegsverhalten hat (Caligari 1995) und in schnell rotierenden Sternen deutlich geringer ausgeprägt ist (Donahue 1993, Rüdiger et al. 1998) wird im Weiteren von einer starren Sternrotation ausgegangen.

Eine langfristige Speicherung von Magnetfeldern innerhalb der Konvektionszone, etwa für die Zeitdauer eines mehrjährigen Aktivitätszyklus, ist praktisch nicht möglich. Im thermischen Gleichgewicht mit ihrer Umgebung $\left(T_{\mathrm{i}}=T_{\mathrm{e}}\right)$ besitzen sie aufgrund des lateralen Druckgleichgewichts (2.26) eine geringere Dichte $\rho_{\mathrm{i}}<\rho_{\mathrm{e}}$ und unterliegen deshalb dem Auftrieb; Flussröhren mit Super-Äquipartitionsfeldstärken ${ }^{1}$ von $B_{0} \gtrsim 10^{5} \mathrm{G}$, auf die beispielsweise Sonnenflecken zurückzuführen sind, durchqueren die Konvektionszone $\left(\sim 2 \cdot 10^{10} \mathrm{~cm}\right)$ mit Aufstiegsgeschwindigkeiten von $\sim 3 \cdot 10^{3} \mathrm{~cm} / \mathrm{s}$ in weniger als 100 d. Dies würde innerhalb kurzer Zeit zu einer gravierenden Abnahme des magnetischen Flusses führen und dem Feldverstärkungsmechanismus seine Grundlage entziehen. Es wird angenommen, dass dieser in der Umgebung der Tachocline arbeitet, der Grenzschicht am unteren Rand der Konvektionszone, die durch starke Gradienten der differentiellen Rotation charakterisiert wird. Das am Übergang zum radiativen Kern — in der stabil geschichteten, subadiabatischen Overshoot-Region — gespeicherte Magnetfeld unterliegt dabei dem turbulenten Einfluss überschießender Plasmaströmungen aus der darüberliegenden Konvektionszone (Spruit \& van Ballegooijen 1982, van Ballegooijen 1982).

Die verschiedenen Bereiche werden durch ihre Superadiabatizität $\delta=\nabla-\nabla_{\text {ad }}$ unterschieden, wobei $\nabla=(\mathrm{d} \ln T / \mathrm{d} \ln p)$ der logarithmische Temperaturgradient ist; der Index 'ad' steht für adiabatische Zustandsänderungen. In der superadiabatisch geschichteten Konvektionszone ist $\delta>0$ und in der subadiabatischen Overshoot-Region $\delta<0$.

\footnotetext{
${ }^{1}$ Die Äquipartitionsfeldstärke $B_{\text {eq }}$ sei die Feldstärke, bei der die magnetische Energiedichte $B^{2} /(8 \pi)$ gleich der kinetischen Energiedichte $\rho v_{\mathrm{c}}{ }^{2} / 2$ ist. Dabei ist $v_{\mathrm{c}}$ eine typische Geschwindigkeit am Ort des Magnetfelds, etwa der konvektiven Strömung oder der differentiellen Rotation.
} 


$$
\begin{array}{cl}
\text { Energiegleichung: } & \frac{d}{d t}\left(\frac{p}{\rho^{\gamma}}\right)=0 \\
\text { Zustandsgleichung: } & p=\rho \frac{\Re}{\mu} T \\
\text { Druckgleichgewicht: } & p_{\mathrm{e}}=p+\frac{B^{2}}{8 \pi}
\end{array}
$$

Fluss-Erhaltung: $\quad \Phi=\mathbf{A} \cdot \mathbf{B}=A B=$ const.

Effektive Gravitation: $\quad \mathbf{g}_{\mathrm{eff}}=-\nabla\left(\Psi_{*}-\frac{1}{2}|\boldsymbol{\Omega} \times \mathbf{r}|^{2}\right)$

Strömungswiderstand: $\quad \mathbf{f}_{\mathrm{D}}=-C_{\mathrm{D}} \rho_{\mathrm{e}} \frac{d}{2} v_{\perp}^{2} \hat{\mathbf{v}}_{\perp}$

Walénsche Gleichung: $\quad \frac{d}{d t}\left(\frac{B}{\rho}\right)=\frac{B}{\rho}\left(\frac{\partial v_{\mathrm{t}}}{\partial s}-\frac{v_{\mathrm{n}}}{R}\right)$

$$
\begin{aligned}
& \frac{d \mathbf{t}}{d t} \cdot \mathbf{n}=\frac{\partial v_{\mathrm{n}}}{\partial s}+\frac{v_{\mathrm{t}}}{R}+\frac{v_{\mathrm{b}}}{R_{\mathrm{t}}} \\
& \frac{d \mathbf{t}}{d t} \cdot \mathbf{b}=\frac{\partial v_{\mathrm{b}}}{\partial s}-\frac{v_{\mathrm{n}}}{R_{\mathrm{t}}}
\end{aligned}
$$

\section{Bewegungsgleichung:}

$$
\begin{aligned}
\rho \frac{d \mathbf{v}}{d t} \cdot \mathbf{t} & =-\frac{\partial p}{\partial s}+\rho \mathbf{g}_{\text {eff }}+2 \rho(\mathbf{t} \times \mathbf{v}) \cdot \boldsymbol{\Omega} \\
& =\left(\rho-\rho_{\mathrm{e}}\right)\left(\mathbf{t} \cdot \mathbf{g}_{\mathrm{eff}}\right)+2 \rho(\mathbf{t} \times \mathbf{v}) \cdot \boldsymbol{\Omega}+\frac{\partial}{\partial s} \frac{B^{2}}{8 \pi} \\
\rho \frac{d \mathbf{v}}{d t} \cdot \mathbf{n} & =\left(\rho-\rho_{\mathrm{e}}\right)\left(\mathbf{n} \cdot \mathbf{g}_{\mathrm{eff}}\right)+2 \rho(\mathbf{n} \times \mathbf{v}) \cdot \boldsymbol{\Omega}+\frac{1}{R} \frac{B^{2}}{4 \pi}+\mathbf{f}_{\mathrm{D}} \cdot \mathbf{n} \\
\rho \frac{d \mathbf{v}}{d t} \cdot \mathbf{b} & =\left(\rho-\rho_{\mathrm{e}}\right)\left(\mathbf{b} \cdot \mathbf{g}_{\mathrm{eff}}\right)+2 \rho(\mathbf{b} \times \mathbf{v}) \cdot \boldsymbol{\Omega}+\mathbf{f}_{\mathrm{D}} \cdot \mathbf{b}
\end{aligned}
$$




\subsubsection{Mechanisches Gleichgewicht}

Ausgangspunkt für alle weiteren Überlegungen ist die Annahme der Existenz isolierter, axialsymmetrischer Flussröhren innerhalb der Overshoot-Region, die im stationären mechanischen Kräftegleichgewicht in Ebenen parallel zum Äquator liegen. Andere Ausgangskonfigurationen (z.B. isotherme Nicht-Gleichgewichtszustände) wurden u.a. von Choudhuri (1989), Moreno-Insertis et al. (1992), Fan et al. (1993, 1994) und Caligari et al. (1998) untersucht.

Außerhalb der Äquatorebene ist es im stationären Gleichgewicht nicht möglich, den Auftrieb in sphärisch-radialer Richtung vollständig zu kompensieren: Die Binormalenkomponente (2.36) der Bewegungsgleichung fordert wegen $\boldsymbol{\Omega} \cdot \mathbf{n}=0$ einen verschwindenden Dichtekontrast,

$$
\Delta \rho=\left(\rho_{\mathrm{i}, 0}-\rho_{\mathrm{e}, 0}\right) \equiv 0 .
$$

Aufgrund der Auftriebsneutralität und Axialsymmetrie ist die Tangentialkomponente (2.33) identisch erfüllt. Im Kräftegleichgewicht wird die nach innen gerichtete magnetische Krümmungskraft durch die Corioliskraft kompensiert, die aus der Strömung $\mathbf{v}_{0}=\mathbf{v}_{\mathrm{i}, 0}-\mathbf{v}_{\mathrm{e}, 0}=v_{0} \mathbf{t}$ mit der Relativgeschwindigkeit

$$
v_{0}=\frac{\Omega}{\kappa_{0}}\left[\sqrt{1+\left(\frac{B_{0}}{\sqrt{4 \pi \rho_{0}}} \frac{\kappa_{0}}{\Omega}\right)^{2}}-1\right]>0
$$

resultiert, wobei $\kappa_{0}=1 / R_{0}$ die konstante Krümmung des Flussrings ist. Das Plasma im Innern der Flussröhre ist etwas kühler und rotiert schneller als die umgebende Konvektionszone - im mit dem Stern rotierenden Bezugssystem gilt bei starrer Rotation $\mathbf{v}_{\mathrm{e}}=0$.

Das mechanische toroidale Gleichgewicht stellt den natürlichen Zustand einer Flussröhre in der Overshoot-Region dar (Moreno-Insertis et al. 1992), der sich etwa aufgrund folgender Entwicklung einstellt (vgl. Abb. 2.1). Angenommen, eine Flussröhre befinde sich in einem Nicht-Gleichgewichtszustand, in dem (o.B.d.A.) die Innendichte geringer als die der Umgebung ist $\left(\rho_{\mathrm{i}}<\rho_{\mathrm{e}}\right)$ und keine Geschwindigkeitsdifferenz $(v=0)$ existiert, so wird sich durch ihren adiabatischen Aufstieg innerhalb der subadiabatischen Overshoot-Region der Dichtekontrast stetig verringern. Die gleichzeitig aufgrund der Drehimpulserhaltung einsetzende interne Strömung $(v<0)$ bewirkt eine Corioliskraft, die zusammen mit der Krümmungskraft den Flussring in Richtung zur Rotationsachse zusammenzieht. Diese beiden Kräfte können aber die Auftriebskomponente parallel zur Rotationsachse nicht kompensieren, weshalb die Flussröhre während ihres Aufstiegs zu höheren Breiten driftet. Innerhalb des Isorotationsradius $\left(\Omega_{\mathrm{i}}=\Omega_{\mathrm{e}}\right.$, d.h. $\left.v=0\right)$ führt die Drehimpulserhaltung aufgrund des kleiner werdenden Krümmungsradius $R$ so lange zu einem Anwachsen der Relativgeschwindigkeit $(v>0)$, bis die Corioliskraft die Krümmungskraft kompensieren und die Flussröhre im auftriebsneutralen Kräftegleichgewicht halten kann, d.h. bis Gl. (2.38) erfüllt ist. Bei nicht zu großer Anfangsfeldstärke liegt der isochore Flussring $\left(\rho_{\mathrm{i}}=\rho_{\mathrm{e}}\right)$ nun etwas näher an der Rotationsachse, aber weiter außen in der Overshoot-Region.

Entdimensionierung der Strömungsgeschwindigkeit $v_{0}:$ In Kap. 4 werden Sterne unterschiedlicher Entwicklungsstufen und Massen untersucht, bei denen sich die Absolut- 


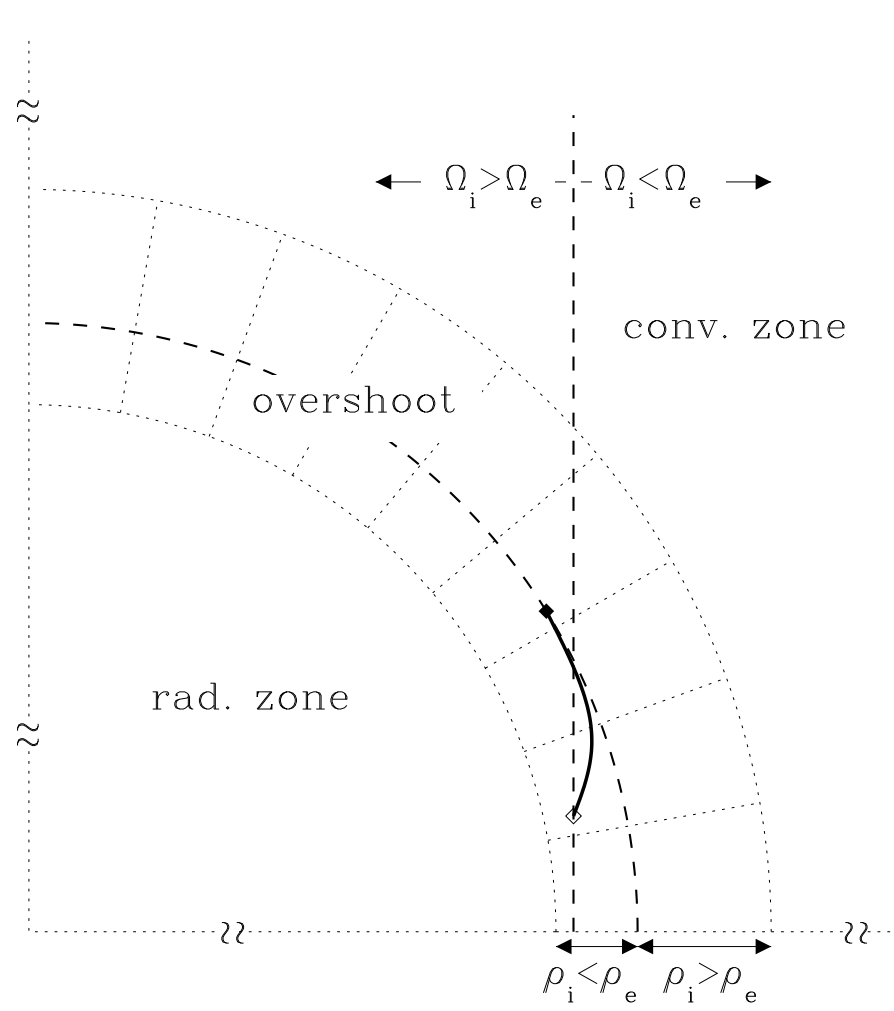

\begin{abstract}
ABBILDUNG 2.1: Übergang einer axialsymmetrischen Flussröhre ins mechanische Kräftegleichgewicht (nach Caligari 1995). Ausgehend von einem Nicht-Gleichgewichtszustand (offene Raute) baut der Flussring beim Aufstieg durch die OvershootRegion den Dichtekontrast zwar $a b$, wird dabei aber zu höheren Breiten und näher an die Rotationsachse gelenkt. Im Gleichgewicht kompensiert die Corioliskraft der internen Strömung $\left(\Omega_{\mathrm{i}}>\Omega_{\mathrm{e}}\right)$ die magnetische Krümmungskraft.
\end{abstract}

werte der Gleichgewichtsgrößen deutlich voneinander unterscheiden. So führen die dort relevanten Parameterbereiche

$$
\begin{aligned}
10^{4} \mathrm{G} & \leq B_{0} \leq 10^{6} \mathrm{G} \\
10^{10} \mathrm{~cm} & \leq R_{0} \leq 3 \cdot 10^{11} \mathrm{~cm} \\
10^{-2} \mathrm{~g} / \mathrm{cm}^{3} & \leq \rho_{0} \leq 4 \cdot 10^{-1} \mathrm{~g} / \mathrm{cm}^{3} \\
2.6 \cdot 10^{-7} \mathrm{~s}^{-1} & \leq \Omega \leq 10^{-4} \mathrm{~s}^{-1}
\end{aligned}
$$

zu einem Wertebereich von $B \kappa /(\sqrt{4 \pi \rho} \Omega) \sim 10^{-4} \ldots 10^{3}$. Es ist daher vorteilhaft, zur Beschreibung des Gleichgewichts die dimensionslose Alfvénsche Machzahl

$$
M_{\alpha 0}=\frac{v_{0}}{c_{\mathrm{a}, 0}}=\frac{\Omega}{c_{\mathrm{a}, 0} \kappa_{0}}\left(\sqrt{1+\left(\frac{c_{\mathrm{a}, 0} \kappa_{0}}{\Omega}\right)^{2}}-1\right)
$$

zu verwenden, welche die interne Strömungsgeschwindigkeit $v_{0}$ in Einheiten der AlfvénGeschwindigkeit

$$
c_{\mathrm{a}}=\frac{B}{\sqrt{4 \pi \rho}}
$$

angibt. Der Verlauf von $M_{\alpha 0}$ ist als Funktion des dimensionslosen Ausdrucks $c_{\mathrm{a}, 0} \kappa_{0} / \Omega$, der praktisch alle gleichgewichtsrelevanten Größen umfasst, in Abb. 2.2 dargestellt: Es ist immer $M_{\alpha}<1$. In der Äquatorebene der Sonne ist in der Mitte der Overshoot-Region beispielsweise (mit $\rho=0.15 \mathrm{~g} / \mathrm{cm}^{3}, \kappa_{0}=1 /\left(5.07 \cdot 10^{10}\right) \mathrm{cm}^{-1}, \Omega_{\odot}=2.76 \cdot 10^{-6} \mathrm{~s}^{-1}$ ) $c_{\mathrm{a}} \kappa_{0} / \Omega=5.2 \cdot 10^{-6} \times B_{0}[\mathrm{G}]$. 


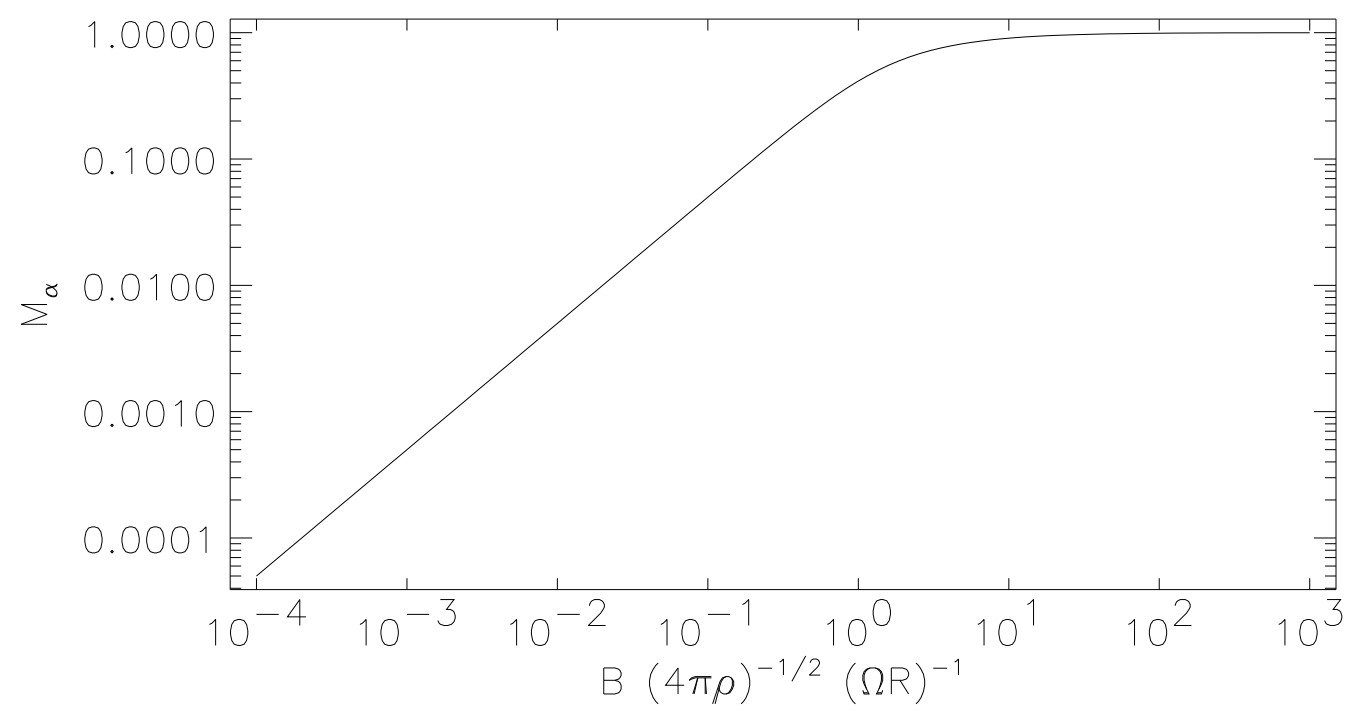

ABBILDUNG 2.2: Alfvénsche Machzahl $M_{\alpha}$ für magnetische Flussröhren im stationären mechanischen Gleichgewicht.

In Hinblick auf die Untersuchung schnell rotierender Doppelsterne ist weiterhin die Rossbyzahl

$$
R o=v_{0} \frac{\kappa_{0}}{\Omega}=\frac{2 M_{\alpha 0}^{2}}{1-M_{\alpha 0}^{2}}
$$

von Interesse, die den relativen Einfluss der Corioliskraft quantifiziert; der magnetostrophe Grenzfall wird beispielsweise durch sehr kleine Rossbyzahlen charakterisiert.

\subsection{Ausbruch magnetischer Flussröhren}

Turbulente Fluktuationen in der Overshoot-Region, hervorgerufen durch überschießende Gaspakete aus der Konvektionszone, bewirken eine Verschiebung der Flussröhrensegmente aus ihrer toroidalen Ausgangskonfiguration zu benachbarten Orten, an denen kein exaktes Kräftegleichgewicht mehr herrscht und die weitere Entwicklung durch die resultierende Nettokraft bestimmt wird. Grundsätzlich reagiert eine Flussröhre auf kleine Störungen mit einem charakteristischen Spektrum von Eigenschwingungen, deren Struktur und Frequenzen von der Krümmung und Feldstärke der Flussröhre sowie den Gegebenheiten der Sternschichtung abhängen. Oberhalb einer kritischen Feldstärke sind kleine Auslenkungen instabil, wachsen an und entwickeln Schleifen, die in der Konvektionszone aufgrund ihres magnetischen Auftriebs bis zur Oberfläche aufsteigen und dort Flecken, bipolare Gruppen und aktive Regionen bilden.

\subsubsection{Instabilitäts-Mechanismen}

Für toroidale Flussröhren ist insbesondere der Instabilitätstyp von Bedeutung, bei dem sie in einer gravitativ geschichteten Umgebung gegenüber adiabatisch ablaufenden Ver- 
biegungen ihrer Feldlinien anfällig sind (Parker 1966). Diese Parker-Instabilität basiert prinzipiell auf folgendem Mechanismus. Wird ein Flussröhrensegment über die Gleichgewichtsposition angehoben, so ändern sich die internen Strömungsverhältnisse dahingehend, dieses Element wieder ins hydrostatische Gleichgewicht zu bringen. Dazu setzt ein (Netto-)Massenfluss aus dem angehobenen Teil zu den tiefer liegenden Segmenten ein, wodurch die Dichte im ausgelenkten Teil zusätzlich zur adiabatischen Expansion abnimmt und den Auftrieb fördert; der letztlich den Auftrieb bestimmende Dichtekontrast $\Delta \rho$ hängt aber auch empfindlich von der Superadiabatizität $\delta$ der Umgebung ab. Nach Gl. (2.19) ändert sich durch die Biegung der Flussröhre gleichzeitig die magnetische Spannung, die mit dem grundsätzlichen Ziel der Verkürzung der Feldlinien dieser Auslenkung entgegenwirkt. Letztlich entscheidet das Verhältnis zwischen Auftriebs- und Krümmungskraft über das Einsetzen der Instabilität.

Unter bestimmten Umständen tritt, insbesondere bei hohen Breiten, die axialsymmetrische 'poleward slip'-Instabilität auf, bei welcher sich der Flussring — ähnlich einem gespannten Gummiband - zusammenzieht und Richtung Pol rutscht. Verantwortlich für dieses Verhalten ist das Überwiegen der nach innen gerichteten magnetischen Krümmungskraft über die nach außen weisende Corioliskraft; trotz der durch die Drehimpulserhaltung anwachsenden Relativgeschwindigkeit $v$ kann diese die starke Spannung des Rings bei kleinen Krümmungsradien nicht mehr kompensieren. Bei den hier untersuchten Fällen ist dieser Mechanismus allerdings nur von untergeordneter Bedeutung.

Im Rahmen dieser Arbeit wurde eine zusätzliche, neuartige Instabilität gefunden, die auf den Strömungswiderstand $\mathbf{f}_{D}$ einer Flussröhre zurückzuführen ist. Bei den hier untersuchten Flussröhren entwickelt sich diese Strömungs-Instabilität auf Zeitskalen, die mit den Anwachszeiten üblicher Parker-Instabilitäten vergleichbar sind, weshalb sie für die Speicherung magnetischer Flussröhren unter bestimmten Bedingungen von Bedeutung ist (vgl. Kap. 10). Die detaillierte Untersuchung ihrer Eigenschaften und des zugrundeliegenden Mechanismus folgt in Kap. 14.

\subsubsection{Lineare Stabilitätsanalyse}

Die Zerlegung infinitesimaler adiabatischer Auslenkungen $\delta \mathbf{r}(s, t)$ einer Flussröhre aus ihrer toroidalen Gleichgewichtslage $\mathbf{r}_{0}(s)$ in ein vollständiges System harmonischer Basisfunktionen der azimutalen Wellenzahl $m$ (vgl. Abb. 2.3),

$$
\mathbf{r}(s, t)=\mathbf{r}_{0}+\delta \mathbf{r}=\mathbf{r}_{0}+\sum_{m=0}^{\infty} \delta \mathbf{r}_{m} \exp \left[i\left(m \kappa_{0} s+\omega_{m} t\right)\right]
$$

und die Bestimmung der Eigenfrequenzen $\omega_{m}$ erfolgt mittels einer linearen Stabilitätsanalyse (Spruit \& van Ballegooijen 1982, Ferriz-Mas \& Schüssler 1993, 1995, FerrizMas et al. 1994). Durch den Versatz $\delta \mathbf{r}$ ergeben sich aus dem Gleichungssystem dünner Flussröhren (Tab. 2.1) für alle Größen $f_{i}$ kleine Abweichungen $\delta f_{i}$ vom Gleichgewichtswert $f_{i, 0}$. Die Kombination der resultierenden Gleichungen und Vernachlässigung von Störungstermen von höherer als 1. Ordnung ( $\propto \prod\left(\delta f_{i}\right)^{n_{i}}$ mit $\sum n_{i} \geq 2$ ) führt zu Bewegungsgleichungen für die lineare Auslenkung $\delta \mathbf{r}$, die nur von den Gleichgewichtsgrößen 

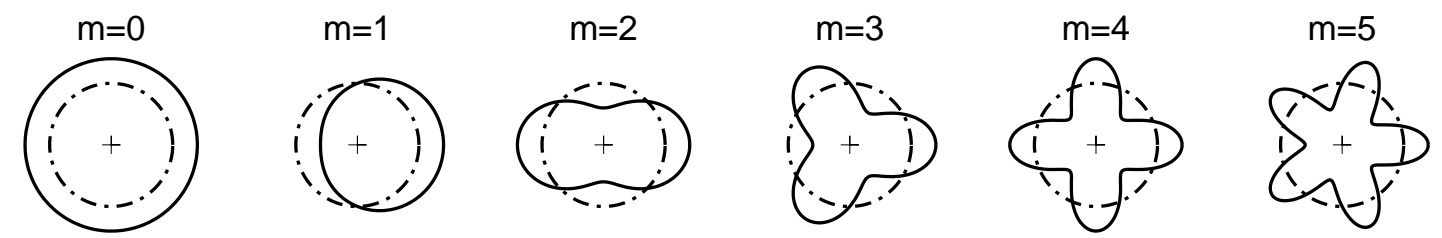

AbBILDUNG 2.3: Schematische Darstellung von Eigenmodi mit azimutalen Wellenzahlen $m=$ $1 \ldots 5$. Kreise (gestrichelt) symbolisieren die toroidale Ausgangskonfiguration der Flussröhre im Gleichgewicht.

$f_{i, 0}$ abhängen und mit der Zerlegung (2.42) in ein algebraisches Gleichungssystem für die Eigenvektoren $\delta \mathbf{r}_{m}$ überführt werden. Nicht-triviale Lösungen dieses Systems existieren nur, wenn die Eigenfrequenz $\omega_{m}$ Lösung der Dispersionsgleichung

$$
\sum_{i=0}^{6} c_{i} \omega^{i}=0
$$

ist. Die Wurzeln dieses Polynoms 6. Grades entsprechen den sechs Freiheitsgraden des Problems: Die prograde und retrograde Ausbreitung periodischer Auslenkungen in drei Raumdimensionen stellen gekoppelte magneto-akustische Transversal- und Longitudinalwellen dar. Die komplexwertige Eigenfrequenz $\omega$ bestimmt die Entwicklung der Flussröhre: Ihr Realteil legt die Schwingungsperiode $T_{\text {is }}=2 \pi / \Re(\omega)$ der Auslenkung und zusammen mit der Wellenzahl $m$ die Ausbreitungsrichtung und Phasengeschwindigkeit

$$
v_{\mathrm{p}}=-\frac{\Re(\omega)}{m \kappa_{0}}
$$

des Wellenmodus fest. Der Imaginärteil $\Im(\omega)$ entspricht dem Inversen der linearen Anwachszeit

$$
\tau=\frac{1}{\Im(\omega)}
$$

für $\Im(\omega)<0$ wächst die Amplitude der Störung mit der Zeit $t$ exponentiell an, für $\Im(\omega)>$ 0 nimmt sie entsprechend $a b$.

Die Angabe einer algebraischen Dispersionsgleichung ist nur im Einzelsternproblem möglich, da hier die Eigenfunktionen des Problems mit den harmonischen Basisfunktionen der Zerlegung (2.42) identisch sind. Im entsprechenden Doppelsternproblem (Kap. 9) bestehen Eigenfunktionen aus einem Spektrum harmonischer Funktionen und können analytisch nicht mehr in geschlossener Form bestimmt werden.

\section{Qualitativer Überblick}

Durch die Vorgabe ihrer Gleichgewichts-Feldstärke $B_{0}$ und -Lage $\left(r_{0}, \lambda_{0}\right)$ im Stern $\left(r_{0}\right.$ : Abstand vom Sternmittelpunkt, $\lambda_{0}$ : Breite) sind die Stabilitätseigenschaften toroidaler Flussringe festgelegt. Sofern keine anderen Angaben gemacht werden, wird als Gleichgewichtsradius $r_{0}$ etwa die Mitte der Overshoot-Region gewählt. Als Beispiel wird in 


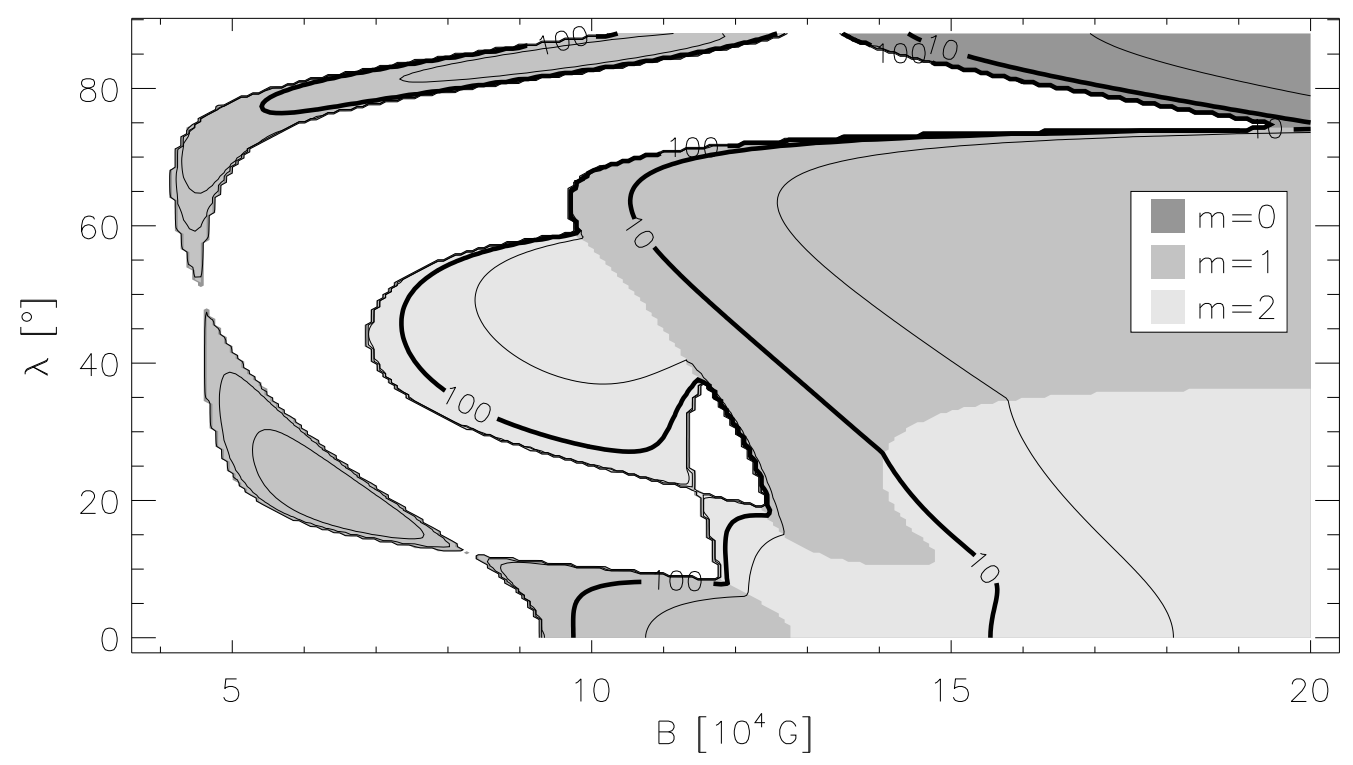

ABBILDUNG 2.4: Anwachszeiten $\tau$ toroidaler Flussröhren im mechanischen Gleichgewicht in einem sonnenähnlichen Einzelstern mit der Rotationsperiode $T=15$ d. $(B, \lambda)$-Konfigurationen für die Parker-Instabilitäten mit azimutalen Wellenzahlen $m=0 \ldots 2$ einsetzen sind grau unterlegt, weiß steht für stabiles Gleichgewicht. Die Niveaulinien markieren Anwachszeiten $\tau=$ $5, \mathbf{1 0}, 50, \mathbf{1 0 0}, 500,1000$ und $5000 \mathrm{~d}$ des jeweils instabilsten Modus einer Ausgangskonfiguration.

Abb. 2.4 das Stabilitätsdiagramm magnetischer Flussringe in einem Einzelstern mit Rotationsperiode $T=15 \mathrm{~d}$ dargestellt ${ }^{2}$. Es zeigt die Anwachszeit $\tau$ des instabilsten Eigenmodus einer $(B, \lambda)$-Konfiguration, der üblicherweise die anfängliche Entwicklung dominiert. Bei Feldstärken $B_{0}<10^{5} \mathrm{G}$ sind Flussröhren überwiegend stabil, es treten lediglich einige lokalisierte Instabilitätsinseln mit meist langen Anwachszeiten auf. Für $B_{0} \gtrsim 10^{5} \mathrm{G}$ findet man hingegen ausgedehnte Bereiche der Parker-Instabilität mit unterschiedlichen dominanten Wellenzahlen, für welche die Anwachszeiten mit zunehmendem $B$ schnell abnehmen und typischerweise bei wenigen hundert Tagen liegen. Bei hohen Breiten $\left(\lambda_{0} \geq 60^{\circ}\right)$ wirkt sich die mit abnehmendem Krümmungsradius $R_{0}=r_{0} \cos \lambda_{0}$ wachsende Spannungskraft der Magnetfeldlinien stabilisierend auf die Flussröhre aus. Bei hohen Feldstärken nimmt deren Einfluss jedoch überhand und führt zur 'poleward slip'-Instabilität mit $m=0$.

Eine Parker-Instabilität mit $T_{\text {is }} \neq 0$ hat den oszillatorischen Charakter einer Überstabilität, d.h. die Störung erzeugt eine Welle, deren Amplitude mit der Zeitskala $\tau$ anwächst. Die axialsymmetrische Instabilität bei hohen Breiten zeigt hingegen als einzige ein monotones Anwachsen. Abbildung 2.5 verdeutlicht den Verlauf der Schwingungsperiode $T_{\text {is }}$ und deren Verhältnis $R_{\mathrm{t}}=\tau / T_{\text {is }}$ zur linearen Anwachszeit. Überwiegend gilt

\footnotetext{
${ }^{2}$ Diagramme für andere Rotationsperioden, aber ansonsten identische Ausgangskonfiguration sind in Abb. $13.3(T=27 \mathrm{~d})$ und Abb. $9.9(T=2 \mathrm{~d})$ angegeben. Die Diagramme in Abb. $9.18(T=0.75 \ldots 10 \mathrm{~d})$ gelten zwar für Doppelsterne, werden aber durch die Parker-Instabilität dominiert. Zusammengefasst verdeutlichen sie die Abhängigkeit der Stabilitätseigenschaften von der Rotationsperiode.
} 


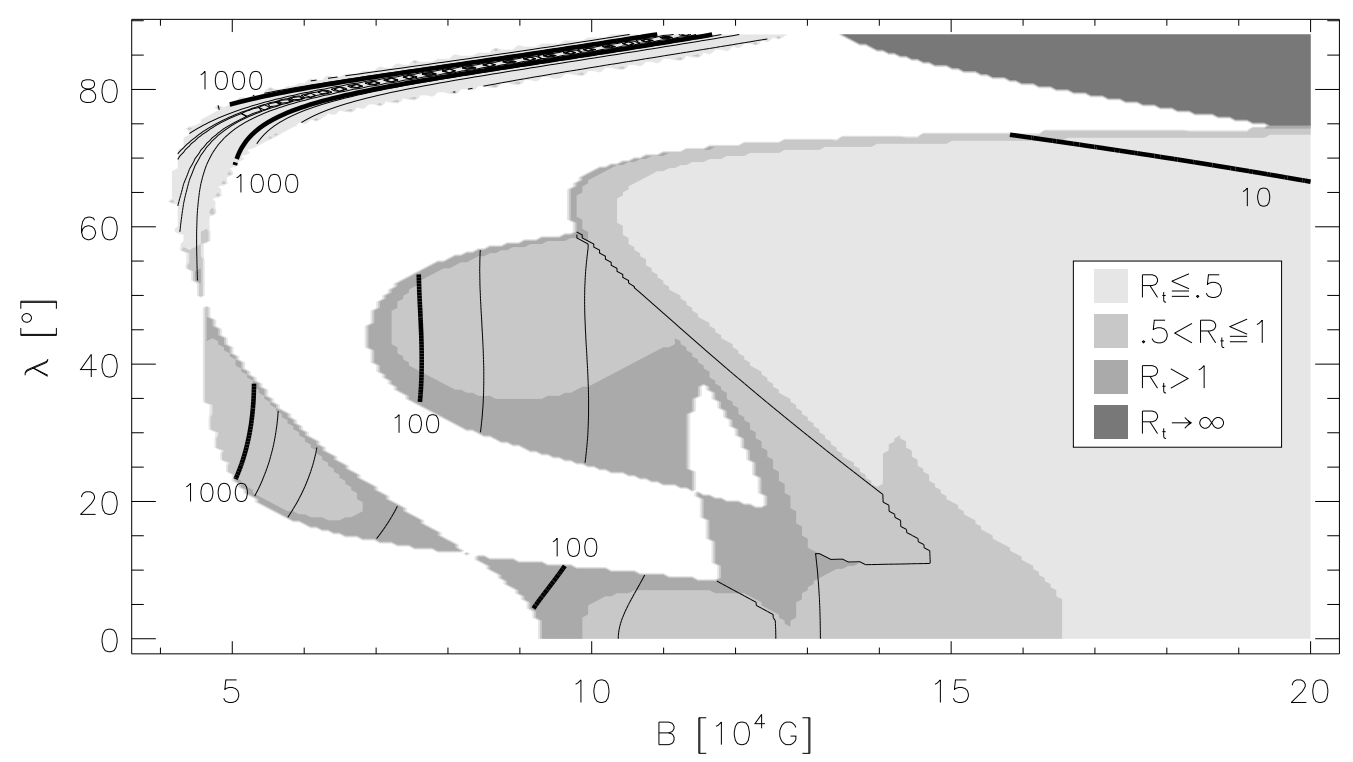

ABBILDUNG 2.5: Schwingungsperioden $T_{\text {is }}$ der in Abb. 2.4 dargestellten Instabilitäten. Die Niveaulinien markieren Schwingungsperioden $T_{\text {is }}=(\mathbf{1 0}, 25,50,75) \cdot 10^{(1,2,3)} \mathrm{d}$ und die grau unterlegten Bereiche das Verhältnis $R_{\mathrm{t}}=\tau / t_{\mathrm{is}}$ zwischen Anwachszeit und Schwingungsperiode. Bei der streng monoton anwachsenden poleward slip-Instabilität (dunkle Ecke rechts oben) ist $T_{\text {is }}=0$ und $R_{\mathrm{t}} \rightarrow \infty$.

$R_{\mathrm{t}} \lesssim 1$, d.h. Teile dieser Flussröhren erreichen die Konvektionszone bereits nach wenigen Schwingungsperioden. Bei hohen Feldstärken ist $R_{\mathrm{t}} \lesssim .5$, sodass Flussröhren trotz Überstabilität mit einer quasi-monoton wachsenden Amplitude aufsteigen.

Die Stabilitätseigenschaften einer Flussröhre werden nachhaltig durch den Wert der Superadiabatizität $\delta$ ihrer Umgebung bzw. den Ausdruck

$$
\beta \delta=\left(\frac{p}{p_{\mathrm{mag}}}\right) \delta \quad \text { mit } \quad p_{\mathrm{mag}}=\frac{B^{2}}{8 \pi}
$$

bestimmt, wobei $p_{\text {mag }}$ der magnetische Druck im Innern der Flussröhre ist. Im Sterninnern gilt typischerweise $\beta \gg 1$, weshalb bereits kleine Änderungen der Superadiabatizität großen Einfluss auf die Stabilitätseigenschaften haben. Im unteren Teil der Konvektionszone variiert $\delta$ vergleichsweise stark; im oben dargestellten Fall ist $d_{\mathrm{os}} \simeq 10^{9} \mathrm{~cm}, \delta=$ $1 \cdot 10^{-6}$ und $H_{\delta} / H_{p} \simeq 0.08$. Dabei ist $d_{\mathrm{os}}$ die Dicke der Overshoot-Region, $H_{p}$ die Druckskalenhöhe und $H_{\delta}$ die Skalenhöhe der Superadiabatizität.

Prinzipiell wird durch die Feldverstärkung im Laufe des Dynamoprozesses die Position einer Flussröhre im Stabilitätsdiagramm auf einer Zeitskala $\tau_{\text {amp }}$ nach rechts verschoben. Falls sie dabei in den Bereich einer Instabilität mit der Anwachsrate $\tau_{\text {is }}$ kommt, hängt ihre weitere Entwicklung vom Verhältnis der Zeitskalen ab: Ist $\tau_{\text {is }} \lesssim \tau_{\text {amp }}$, so wird die Flussröhre ausbrechen und dabei nicht mehr signifikant verstärkt. Für $\tau_{\text {is }} \gg \tau_{\text {amp }}$ wird hingegen während des Anwachsens der Störung auch die Feldstärke zunehmen und sich die Flussröhre im Stabilitätsdiagramm weiter nach rechts bewegen. Dadurch ist es ihr möglich, Instabilitätsinseln zu durchqueren und wieder stabile Zustände bei höheren Feldstärken zu erreichen. Zur klaren Ausprägung eines Aktivitätszyklus müssen die 
Ausbruchszeiten magnetischer Flussröhren kürzer als die Zyklusdauer sein, die bei sonnenähnlichen Sternen im Bereich von etwa 5 .. 15 yr liegen (z.B. Baliunas \& Vaughan 1985). Die Zeitskala $\tau_{\text {is }}$, welche lediglich die anfängliche Entwicklung der Flussröhre in der Overshoot-Region charakterisiert, muss dementsprechend nochmals deutlich kürzer als dieser Zeitrahmen sein. Im Fall der Sonne geht man davon aus, dass der Verstärkungsmechanismus durch starke Scherströmungen am unteren Rand der Konvektionszone angetrieben wird und nimmt für diesen Vorgang als charakteristische Zeitskala mehrere Sonnenrotationen an, d.h. wenige hundert Tage.. Zahlreiche Arbeiten (Choudhuri \& Gilman 1987, Choudhuri 1989, Moreno-Insertis et al. 1992, Fan et al. 1994, Schüssler et al. 1994, Ferriz-Mas 1996) kommen übereinstimmend zu dem Schluss, dass die Ausgangsfeldstärke in der solaren Overshoot-Region etwa eine Größenordnung über der Äquipartitionsfeldstärke $B_{\text {eq }} \approx 10^{4} \mathrm{G}$ liegen muss, damit die Ausbruchscharakteristika magnetischer Flussröhren (Ausbruchsbreite, Kippwinkel, Asymmetrie,... ) konsistent mit Beobachtungen von Sonnenfleckengruppen sind. In der Tat führt die Forderung nach SuperÄquipartitionsfeldstärken $\gtrsim 10^{5} \mathrm{G}$ auf Anwachszeiten von $\sim 100 \ldots 300 \mathrm{~d}$. Unserem Grundgedanken folgend, wird auf diesen bisherigen Erkenntnissen im Fall der Sonne aufgebaut und bei der weiteren Anwendung von vergleichbaren Anwachszeiten ausgegangen.

\subsubsection{Nicht-lineare Entwicklung und Ausbruch}

\section{Numerisches Verfahren}

Die Simulation der nicht-linearen Entwicklung einer geschlossenen dünnen Flussröhre in der äußeren Konvektionszone eines Sterns basiert auf einem numerischen Code, der ursprünglich von Moreno-Insertis $(1984,1986)$ entwickelt und danach von Caligari (1991, 1995) erheblich erweitert wurde.

Das Flussröhren-Programm verwendet ein Lagrangeverfahren, bei dem die Entwicklung einer festen Anzahl von Röhrenelementen konstanter Masse verfolgt wird. Dies ermöglicht die Untersuchung ihrer Dynamik über etliche Druckskalenhöhen hinweg, vom Boden der Konvektionszone bis dicht unter die Sternoberfläche. Zur Parametrisierung der Flussröhre bietet sich die Lagrangesche Masse $\mathrm{d} \bar{m}=\rho A \mathrm{~d} s$ an, mit deren Hilfe die Walénsche Gleichung (2.11) in der Form

$$
\frac{1}{\Phi} \frac{d}{d t}\left(\frac{\mathbf{B}}{\rho}\right)=\frac{d}{d t}\left(\frac{1}{\rho A} \mathbf{t}\right)=\frac{d \mathbf{l}}{d t}=\frac{\partial \mathbf{v}}{\partial \bar{m}}=\frac{1}{A B} \frac{B}{\rho} \frac{\partial \mathbf{v}}{\partial s}=\frac{1}{\Phi}\left(\frac{\mathbf{B}}{\rho} \cdot \nabla\right) \mathbf{v}
$$

geschrieben wird; die spezifische Länge $l=|\mathbf{l}|=1 /(\rho A)=\mathrm{d} s / \mathrm{d} \bar{m}$ ist ein Maß für die Streckung eines Röhrenelements. In dem zweistufigen Differenzenverfahren erfolgt die räumliche Diskretisierung der eindimensionalen Flussröhre auf einem versetzten Gitter (staggered mesh): Mit Gl. (2.47) folgt aus der Ableitung des Geschwindigkeitsvektors v nach der Lagrangeschen Masse $\bar{m}$ an den Gittergrenzen $\mathbf{r}$ die zeitliche Änderung des spezifischen Längenvektors 1 am Gittermittelpunkt eines Massenelements; aus diesen drei Größen kann dann die weitere Dynamik abgeleitet werden. Das ebenfalls zweistufige, implizite zeitliche Integrationsverfahren zeichnet sich dabei durch seine numerische Stabilität aus. 


\section{Simulationen}

Die Anregung der Instabilität in der Overshoot-Region erfolgt durch die Störung des toroidalen Ausgangszustands mit einer Superposition harmonischer Auslenkungen mit Wellenzahlen $m=1 \ldots 5$. Mit dem Einsetzen der Instabilität bilden sich — je nach dominierendem Instabilitätsmodus - $m$ Flussröhren-Schleifen, deren Amplitude mit einer Anwachszeit der Größenordnung $\tau$ zunimmt. Aufgrund des wellenartigen Charakters der Parker-Instabilität bewegt sich die Störung entlang der Flussröhre. Mit dem Massenfluss aus den Gipfelsegmenten in die tiefer liegenden Teile der Flussröhre werden diese schwerer und sinken solange ab, bis sie von der starken Subadiabatizität des radiativen Kerns gestoppt werden. Dieser in der Overshoot-Region verbleibende Teil stellt für die aufsteigende Flussröhre eine effektive Verankerung dar.

Nach ihrem Eintritt in die superadiabatische Schichtung der Konvektionszone beschleunigt sich ihr Aufstieg aufgrund des konvektiven Auftriebs erheblich. Durch den Einfluss der geometrieabhängigen Krümmungskraft und der geschwindigkeitsabhängigen Coriolis- und Reibungskräfte wird die Trajektorie des Gipfelelements typischerweise zu höheren Breiten $\lambda$ und, der Drehimpulserhaltung und dem Wellencharakter der Instabilität entsprechend, in azimutaler Richtung $\phi$ abgelenkt; in einem Doppelstern unterliegt sie zusätzlich der ortsabhängigen Gezeitenkraft. Die Transversalbewegung der Röhre wird durch den Strömungswiderstand der Röhre grundsätzlich gehemmt. Allerdings zeigt sich, dass dessen Einfluss, der mit wachsendem Röhrendurchmesser abnimmt, bei dem üblicherweise verwendeten anfänglichen Wert von $d_{0}=2 \cdot 10^{8} \mathrm{~cm}$ nur einen geringen Einfluss auf die Aufstiegsgeschwindigkeit hat. Der von ihm ausgehende Drehimpuls- und Energietransfer beeinflusst jedoch die internen Strömungsverhältnisse und verursacht u.a. das Einsetzen der Strömungsinstabilität.

Durch die Abnahme des externen Gasdrucks dehnt sich der aufsteigende Teil der Flussröhre stark aus, weshalb die Bedingung (2.16) nahe der Oberfläche $\left(r \gtrsim 0.98 \mathrm{R}_{*}\right)$ gewöhnlich nicht mehr erfüllt ist und die Simulation gestoppt wird. Der eigentliche Ausbruch an der Oberfläche kann daher im Rahmen der Näherung dünner Flussröhren numerisch nicht nachvollzogen werden. Da der Aufstieg hier sehr schnell und nahezu radial verläuft, kann man aber davon ausgehen, dass die Endposition der Simulation mit der tatsächlichen Ausbruchsposition an der Oberfläche gut übereinstimmt.

Dieses Modell ausbrechender Flussröhren führt im Fall der Sonne (Caligari et al. 1995, Schüssler et al. 1996) und bei schnell rotierenden, jungen Sternen (Granzer et al. 2000) zu guten Übereinstimmung mit den Beobachtungen. 


\section{Teil I}

\section{Riesensterne}





\section{Kapitel 3}

\section{Einleitung und Motivation}

\section{Grenzlinien im Hertzsprung-Russell Diagramm}

Beobachtungen charakteristischer Signaturen einer Vielzahl von Sternen geben Hinweise auf die Existenz von Grenzlinien im Hertzsprung-Russell Diagramm (HRD), die Bereiche bestimmter Spektraltypen und Leuchtkraftklassen abteilen, in denen ein charakteristisches Merkmal besonders deutlich ausgeprägt ist oder sich auffällig ändert (Abb. 3.1).

Bei der Analyse der Asymmetrie von Linienprofilen fand man im HRD eine Grenzlinie, an der sich das photosphärische Geschwindigkeitsfeld der Sterne ändert. Ausgehend etwa vom Spektraltyp F0 auf der Hauptreihe verläuft die Granulationsgrenzlinie nahezu vertikal durch alle Leuchtkraftklassen (Gray \& Nagel 1989), wobei kühlere Sterne typischerweise sonnenähnliche Asymmetrien in ihren Spektrallinien zeigen. In kühlen Zwergsternen mit Oberflächentemperaturen $T_{\text {eff }} \lesssim 7000 \mathrm{~K}$ und Massen $M_{*} \lesssim 1.3 \mathrm{M}_{\odot}$ erfolgt der Energietransport in den äußeren Schichten durch Konvektion, die sich am Übergang zur stellaren Photosphäre durch ein typisches Granulationsmuster bemerkbar macht. Je geringer die Sternmasse, desto tiefer ist die Konvektionszone im Vergleich zum Gesamtradius des Sterns: Bei der Sonne (Spektraltyp G2V) sind es etwa 30\%, während sehr massearme M-Zwerge vollständig konvektiv sind. In massereicheren $\left(M>1.3 \mathrm{M}_{\odot}\right)$ Zwergsternen erfolgt der Energietransport in den äußeren Schichten hingegen durch Strahlung. Erst wenn sie die Hauptreihe nach Verbrennen ihres zentralen Wasserstoffvorrats verlassen haben und sich zu kühlen Unterriesen bzw. Riesen entwickeln, bilden sie (nach Überqueren der Granulationsgrenze) eine äußere Konvektionszone, die in Sternen am Fuße des Riesenastes (RGB, red giant branch) bis zu 90\% des Sternradius ausmacht und über $70 \%$ der Sternmasse enthält.

Kühle Sterne, die während ihres sehr langen Hauptreihenstadiums magnetisch aktiv sind, erfahren während dieser Zeit eine signifikante Abbremsung ihrer Rotation (magnetic braking). Vom Stern abströmendes Plasma folgt innerhalb eines bestimmten Bereichs dem Verlauf des dynamisch dominierenden stellaren Magnetfelds und wird von diesem näherungsweise zur Korotation mit der Sternumdrehung gezwungen. Aufgrund des größeren Abstands von der Rotationsachse erhöht sich dadurch der Drehimpuls des Gases, während der des Sterns entsprechend abnimmt. Wenn die spezifische Bewegungsenergie größer als die Energiedichte des stellaren Magnetfelds wird (am so genannten Alfénradius), bewegt sich das Plasma weitgehend unabhängig vom Magnetfeld und transportiert beim 


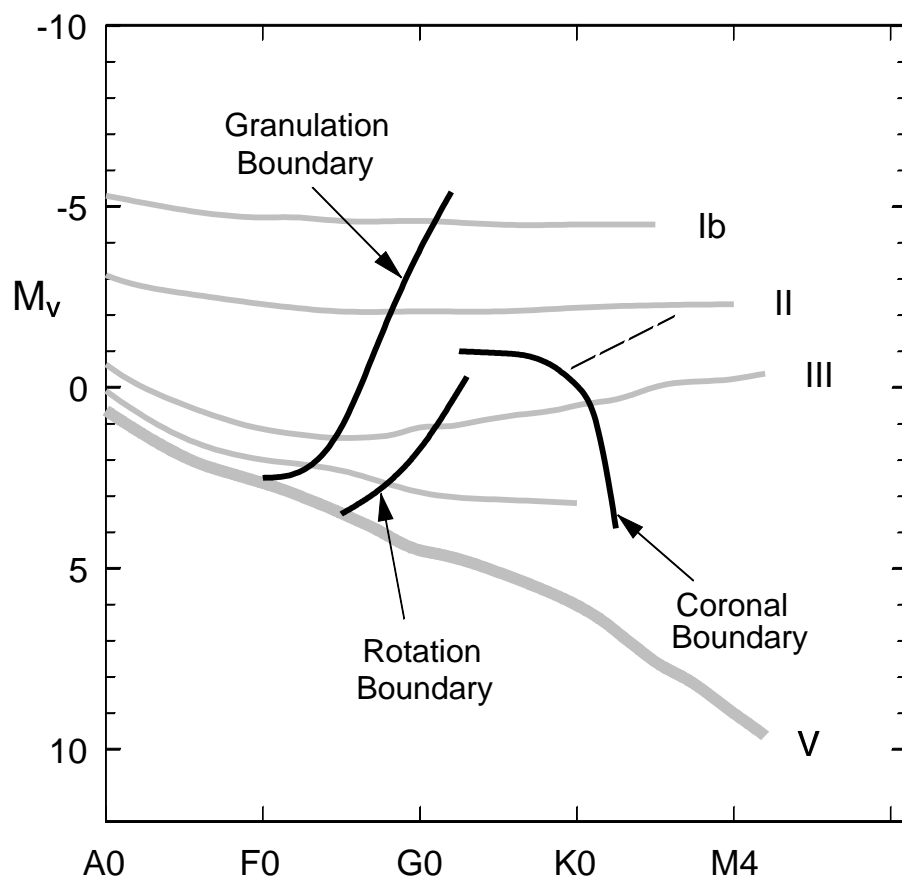

ABBILDUNG 3.1: Schematischer Verlauf verschiedener Grenzlinien im HR Diagramm [nach Gray (1991)]. Die grauen Linien markieren Bereiche verschiedener Leuchtkraftklassen, die etwas dickere entspricht der Hauptreihe (LC V). Grenzenlinien (schwarz) unterteilen das Diagramm in Gebiete, in denen charakteristische Merkmale wie z.B. Konvektion, schnelle Rotation oder starke Röntgenemission mehr oder weniger ausgeprägt sind.

Abströmen den zuvor übertragenen Drehimpuls vom Stern weg. Durch diesen kontinuierlichen Drehimpulsverlust, dessen Ausmaß letztlich von der Stärke des Windes und des Magnetfeldes abhängt, wird die Sternrotation abgebremst. Hauptreihensterne heißer als Typ F zeigen praktisch keine magnetische Aktivität und ändern erst während ihrer Nach-Hauptreihen-Expansion aufgrund des anwachsenden Trägheitsmoments ihre Rotationsrate. Generell scheint eine signifikante Rotationsbremsung bei Zwergen, Unterriesen und Riesen erst auf der kühleren Seite der Rotationsgrenze (Abb. 3.1), nach der Ausbildung einer ausreichend tiefen Konvektionszone und entsprechend starker magnetischer Aktivität, wirksam zu sein. Die Rotationsgrenze verläuft etwa vom Spektraltyp F5 V auf der Hauptreihe bis G0 III bei den Riesen (Gray 1989). Durch die starke Ausdehnung der konvektiven Hülle und die Änderung der Massen-und Drehimpulsverteilung zwischen Hülle und Kern ändert sich sowohl der innere Aufbau als auch die Rotation der NachHauptreihensterne nachhaltig.

\section{Die Koronagrenzlinie}

Mit Satelliten-Missionen wie Einstein, IUE oder ROSAT konnten umfassende Beobachtungen im EUV und weichen Röntgenbereich durchgeführt werden, die bei den meisten Riesensternen Linienemission mehrfach ionisierter Elemente wie C II-IV, Si III-IV oder N V und Röntgenemission bei $1 \ldots 2 \mathrm{keV}$ offenbarten, die charakteristisch für Temperaturen von $10^{4} \ldots 10^{7} \mathrm{~K}$ und typisch für heiße Chromosphären, Übergangsschichten und Koronen sind.

Frühe Beobachtungen (Linsky \& Haisch 1979, Ayres et al. 1981) entdeckten etwa beim Spektraltyp K0-3 eine markante Koronagrenzlinie (CDL, coronal dividing line), die Riesensterne mit Anzeichen für Chromosphären und Koronen von solchen mit chromosphärischen Temperaturen bis etwa $10^{4} \mathrm{~K}$ trennt, die offenbar keine so heißen Atmo- 
sphärenschichten haben. Nachfolgende Beobachtungen bestätigten den drastischen Rückgang koronaler Röntgenemission bei Riesen und untermauerten dadurch das Konzept der CDL (Maggio et al. 1990, Haisch et al. 1990). Zwar waren diese frühen Beobachtungen noch durch eine vergleichsweise geringe Zahl an Objekten und niedrige Nachweisgrenzen limitiert, aber auch die leistungsfähigere ROSAT Durchmusterung (RASS, ROSAT All-Sky Survey) zeigte eine klare Zweiteilung bei der Röntgenemission kühler Riesen(Haisch et al. 1991, 1992a, Ayres et al. 1991). Letztlich war aber auch deren Nachweisempfindlichkeit nicht ausreichend, um die mögliche Existenz von heißem, koronalem Gas in kühlen Riesen mit Bestimmtheit auszuschließen. Zumindest die Beobachtung von Röntgenemission so genannter Hybrid-Sterne ${ }^{1}$ zeigte, dass die CDL nicht auf den Leuchtkraftklassenbereich der hellen Riesen und Überriesen übertragen werden kann (Haisch et al. 1992b, Reimers \& Schmitt 1992, Reimers et al. 1996). Im Folgenden bestätigten wesentlich empfindlichere ROSAT-Beobachtungen den starken Rückgang koronaler Röntgenemission bei Riesensternen um den Spektraltyp K3 (Hünsch et al. 1996), entdeckten dabei aber auch schwache Röntgenstrahlung bei kühleren Riesen, teilweise sogar bei Vertretern des Spektraltyps M (Hünsch et al. 1998). Durch diese Beobachtungen wird das Konzept der CDL zwar aufgeweicht, der signifikante Abfall charakteristischer Röntgenemission in Riesensternen in der Umgebung der Koronagrenze im HRD ist aber nach wie vor gegeben.

\section{Koronale Magnetfelder}

Magnetfelder haben für die Koronen kühler Sterne eine fundamentale Bedeutung. Temperaturen von über $10^{6} \mathrm{~K}$ entsprechen sehr großen thermischen Geschwindigkeiten, die aufgrund der geringen Schwerkraft in der Atmosphäre eines Riesensterns zum Abströmen von Plasma und zum Verlust der Korona führen würden. Weil aber Strömungen senkrecht zur Feldlinie unterbunden werden, kann Plasma, das innerhalb geschlossener Magnetfeldstrukturen gefangen ist, die Korona nicht oder zumindest nur sehr schwer verlassen. Ohne den Einfluss ausgedehnter geschlossener Strukturen würden Teilchen innerhalb kürzester Zeit das Schwerefeld des Sterns überwinden und als Wind abströmen, so wie es bei der Sonne in Zonen 'offener' Feldlinien der Fall ist. Darüber hinaus spielt das Magnetfeld, wie im Fall der Sonne, bei der Heizung eine wichtige Rolle. Durch den Energietransport entlang magnetischer Flussröhren in Form magnetohydrodynamischer Wellen und die Ohmsche Dissipation elektrischer Ströme trägt das Magnetfeld entscheidend zur Aufrechterhaltung koronaler Temperaturen bei.

Die koronale Röntgenemission entsteht hauptsächlich in großskaligen, geschlossenen Magnetfeldstrukturen. Daher führen Antiochos et al. (1986) den starken Emissionsrückgang beim Überqueren der CDL auf das Verschwinden dieser Strukturen zurück, die aufgrund der abnehmenden atmosphärischen Druckskalenhöhe im Laufe der NachHauptreihen-Expansion instabil werden, in kleine, kühlere Bögen übergehen und ihre Energie bei längeren Wellenlängen emittieren. In engem Zusammenhang dazu steht auch der Vorschlag von Ayres et al. (1998), dass die Emission tiefliegender koronaler Bögen

\footnotetext{
${ }^{1}$ Unter Hybrid-Sterne versteht man Sterne des Spektraltyps KII und GII-Ib, die einerseits Linienemission zeigen, wie sie für heiße Übergangsschichten typisch sind, andererseits aber auch Absorptionsmerkmale vorweisen, die auf die Existenz von starken, kühlen Sternwinden hinweisen.
} 
durch zirkumstellares Material verdeckt wird. Ein alternativer Erklärungsansatz führt die CDL auf Sternpopulationen unterschiedlicher Massenbereiche zurück, deren Entwicklungswege im kühlen Riesenstadium zwar dicht beieinander liegen, aber aufgrund ihrer verschieden starken magnetischen Aktivität im Hauptreihenstadium unterschiedliche Rotationsentwicklungen haben (Ayres et al. 1981, 1991, Reimers et al. 1996, Hünsch \& Schröder 1996): Aufgrund ihrer starken magnetischen Abbremsung auf der Hauptreihe ist für Sterne mit Massen $M_{*} \lesssim 1.3 \mathrm{M}_{\odot}$ die Rotationsrate im Riesenstadium so gering, dass der Dynamomechanismus stark zurückgeht und letztlich verschwindet. Massenreichere Sterne erfahren hingegen erst während ihrer Entwicklung durch die HertzsprungLücke nach der Ausbildung einer Konvektionszone und magnetischer Aktivität eine Abbremsung, sodass sie beim Erreichen des RGB noch ausreichend Drehimpuls besitzen um einen Dynamoprozess und Röntgenaktivität aufrecht zu halten. Die CDL wäre in diesem Sinne lediglich der Entwicklungsweg eines etwa $1.3 \mathrm{M}_{\odot}$-Sterns, der auf der Hauptreihe den Übergang zwischen Sternen mit bzw. ohne äußere Konvektionszone repräsentiert. Rosner et al. (1991, 1995) und Kashyap et al. (1994) vermuten hingegen eine Änderung des dominanten Dynamomechanismus während der Nach-Hauptreihen-Entwicklung als Ursache, die zu einem Übergang der atmosphärischen Magnetfeldstruktur von großen, koronalen Bögen zu kleineren Bögen führt, die in Gebiete offener Magnetfeldlinien eingebettet sind. Da die Koronagrenze in der Tat in den Bereich des HRD fällt, in dem auch das Einsetzen massiver Sternwinde beobachtet wird (Reimers 1977), liegt es nahe, als Ursache für dieses Verhalten - in Analogie zu den magnetisch offenen und geschlossenen Gebieten auf der Sonne - eine Änderung der koronalen Magnetfeldtopologie anzunehmen.

Um festzustellen, ob sich eine grundlegende Änderung der atmosphärischen Magnetfeldstruktur in Riesensternen im Rahmen des solaren Modells erklären lässt, wird die Stabilität, Dynamik und Entwicklung magnetischer Flussröhren in Nach-Hauptreihensternen verschiedener Masse und Entwicklungsstadien bis etwa zum Fuße des 'Riesenastes' untersucht (vgl. Holzwarth \& Schüssler 2001). 


\section{Kapitel 4}

\section{Dynamik magnetischer Flussröhren in Riesensternen}

\subsection{Sternmodelle}

\subsubsection{Entwicklungs-Sequenzen}

Zur Erzeugung der Entwicklungssequenzen bzw. einzelner Sternmodelle wird eine erweiterte Version des Sternentwicklungsprogramms von Kippenhahn et al. (1967) verwendet; für eine ausführliche Beschreibung des Codes und seiner Erweiterungen wird auf Granzer (2000) verwiesen. Alle Rechnungen basieren auf OPAL Opazitäten (Iglesias \& Rogers 1996), die mit den atomaren und molekularen Opazitäten von Alexander \& Ferguson (1994) zu tieferen Temperaturen hin ergänzt wurden. Neben der OPAL Zustandsgleichung von Rogers et al. (1996) wurde auch eine analytische, Saha-ähnliche Zustandsgleichung von Gautschy (2001) verwendet. Die thermonuklearen Energieerzeugungs- und Reaktionsraten wurden bei Caughlan \& Fowler (1988) und Clayton (1968) entnommen. Die Beschreibung der Konvektion folgt der (lokalen) Mischungswegtheorie mit einem Mischungswegparameter $\alpha=1.67$. Die ursprüngliche relative Elementhäufigkeit (bezüglich der Masse) von Wasserstoff $(X)$, Helium $(Y)$ und schwereren Elementen $(Z)$, wurde zu $X=0.703, Y=0.277$ und $Z=0.02$ bestimmt (vgl. Granzer 2000). Zusätzlich wurden Sternmodelle verwendet, die mit dem Geneva-Toulouse-Entwicklungsprogramm erzeugt (z.B. Charbonnel \& Talon 1999) und dankenswerterweise von C. Charbonnel zur Verfügung gestellt wurden (Schüssler et al. 2001). Abbildung 4.1 zeigt in einem theoretisches HR Diagramm einen Vergleich zwischen Entwicklungssequenzen von Sternen mit Massen zwischen $M_{*}=1 \ldots 3 \mathrm{M}_{\odot}$ während ihrer Nach-Hauptreihen-Entwicklung. In der weiteren Untersuchung werden überwiegend die auf der Gautschy-Zustandsgleichung basierenden Entwicklungssequenzen verwendet; die Zustandsgrößen ausgewählter Sternmodelle sind in Tab. 4.1 zusammengefasst.

Ein sonnenähnlicher Dynamomechanismus basiert auf der Existenz einer äußeren Konvektionszone. Während Sterne mit Massen $M_{*} \lesssim 1.3 \mathrm{M}_{\odot}$ diese bereits in ihrem Hauptreihenstadium besitzen, bilden massereichere Sterne erst während ihrer NachHauptreihen-Entwicklung eine konvektive Hülle: In den hier berechneten Modellen setzt Konvektion in einem $1.5 \mathrm{M}_{\odot}$-Stern etwa bei einer Temperatur $T_{\text {eff }} \simeq 6000 \mathrm{~K}$ ein; diese 


\begin{tabular}{|c|c|c|c|c|c|}
\hline $\begin{array}{c}M_{*} \\
{\left[\mathrm{M}_{\odot}\right]}\end{array}$ & $\mathrm{Nr}$. & $\begin{array}{l}\text { Alter } \\
{[\mathrm{Gyr}]}\end{array}$ & $\begin{array}{r}L_{*} \\
{\left[\mathrm{~L}_{\odot}\right]}\end{array}$ & $\begin{array}{l}T_{\text {eff }} \\
{[\mathrm{K}]}\end{array}$ & $\begin{array}{r}R_{*} \\
{\left[\mathrm{R}_{\odot}\right]}\end{array}$ \\
\hline \multirow[t]{8}{*}{1.0} & 465 & 11.93 & 2.35 & 4854 & 2.17 \\
\hline & 480 & 11.98 & 2.58 & 4820 & 2.31 \\
\hline & 495 & 12.03 & 2.83 & 4798 & 2.44 \\
\hline & 510 & 12.08 & 3.07 & 4782 & 2.56 \\
\hline & 525 & 12.11 & 3.33 & 4769 & 2.68 \\
\hline & 540 & 12.14 & 3.59 & 4759 & 2.79 \\
\hline & 585 & 12.21 & 4.30 & 4735 & 3.09 \\
\hline & 675 & 12.32 & 6.02 & 4690 & 3.72 \\
\hline \multirow[t]{5}{*}{1.5} & 480 & 2.447 & 6.42 & 4958 & 3.44 \\
\hline & 510 & 2.478 & 7.25 & 4919 & 3.71 \\
\hline & 570 & 2.518 & 8.67 & 4877 & 4.13 \\
\hline & 600 & 2.535 & 9.44 & 4858 & 4.35 \\
\hline & 660 & 2.563 & 11.05 & 4825 & 4.76 \\
\hline \multirow[t]{6}{*}{2.0} & 374 & 0.9161 & 14.68 & 4986 & 5.14 \\
\hline & 376 & 0.9171 & 15.64 & 4963 & 5.36 \\
\hline & 378 & 0.9179 & 16.47 & 4946 & 5.54 \\
\hline & 382 & 0.9195 & 18.08 & 4918 & 5.87 \\
\hline & 386 & 0.9210 & 19.62 & 4895 & 6.17 \\
\hline & 388 & 0.9215 & 20.23 & 4886 & 6.29 \\
\hline \multirow[t]{6}{*}{2.5} & 646 & 0.4784 & 33.49 & 4940 & 7.91 \\
\hline & 647 & 0.4787 & 36.30 & 4908 & 8.35 \\
\hline & 649 & 0.4791 & 41.31 & 4862 & 9.07 \\
\hline & 650 & 0.4792 & 43.60 & 4844 & 9.39 \\
\hline & 653 & 0.4795 & 47.17 & 4818 & 9.88 \\
\hline & 655 & 0.4797 & 49.44 & 4802 & 10.18 \\
\hline \multirow[t]{5}{*}{3.0} & 466 & 0.2913 & 75.28 & 4817 & 12.48 \\
\hline & 468 & 0.2915 & 89.95 & 4748 & 14.04 \\
\hline & 469 & 0.2916 & 96.59 & 4721 & 14.71 \\
\hline & 470 & 0.2917 & 103.37 & 4697 & 15.38 \\
\hline & 471 & 0.2918 & 110.38 & 4673 & 16.06 \\
\hline
\end{tabular}

TABELLE 4.1: Zustandsgrößen von Riesensternen unterschiedlicher Masse und Entwicklungsstadien. 


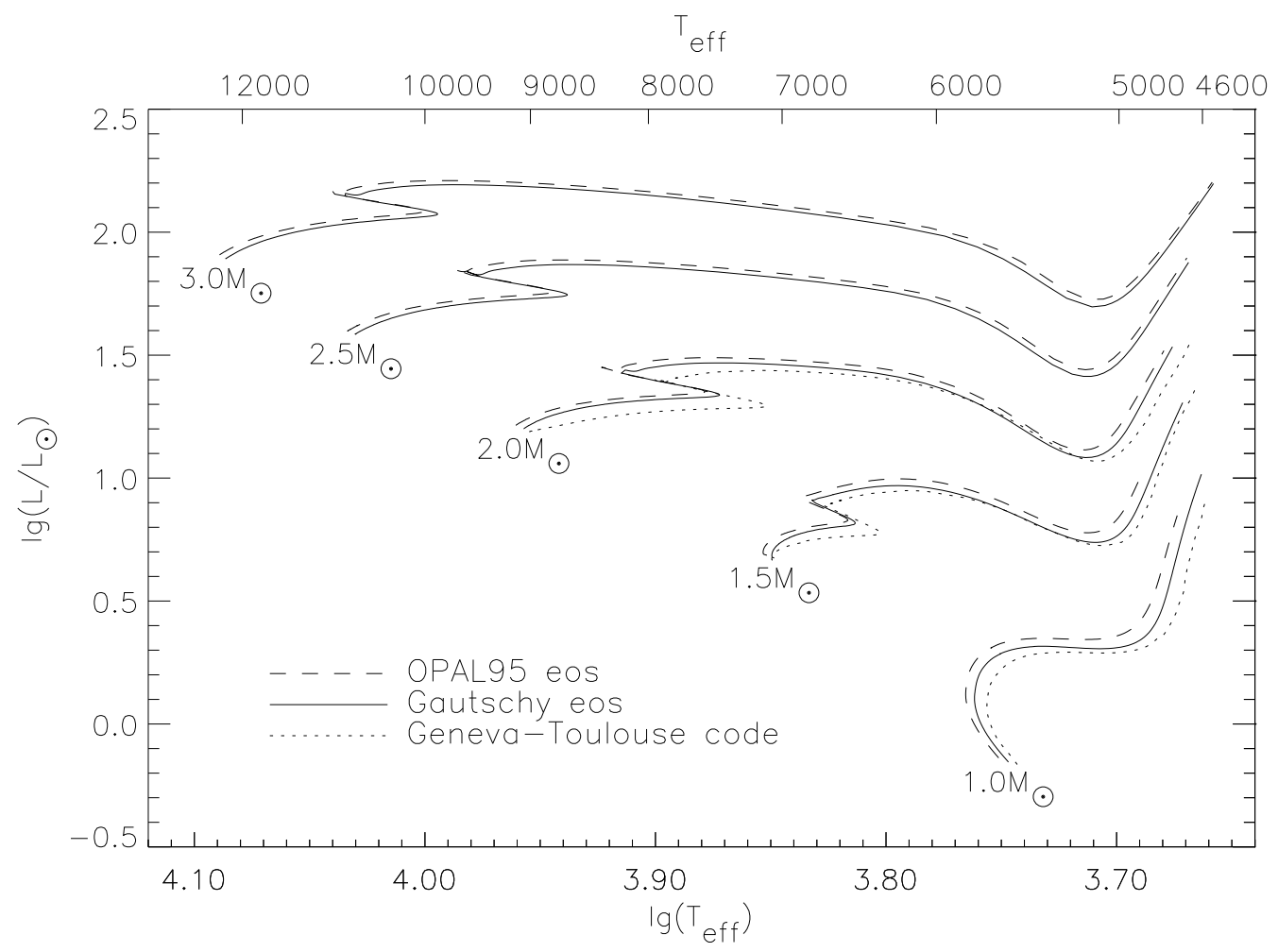

AbBILDUNG 4.1: Vergleich verschiedener Nach-Hauptreihen-Entwicklungssequenzen.

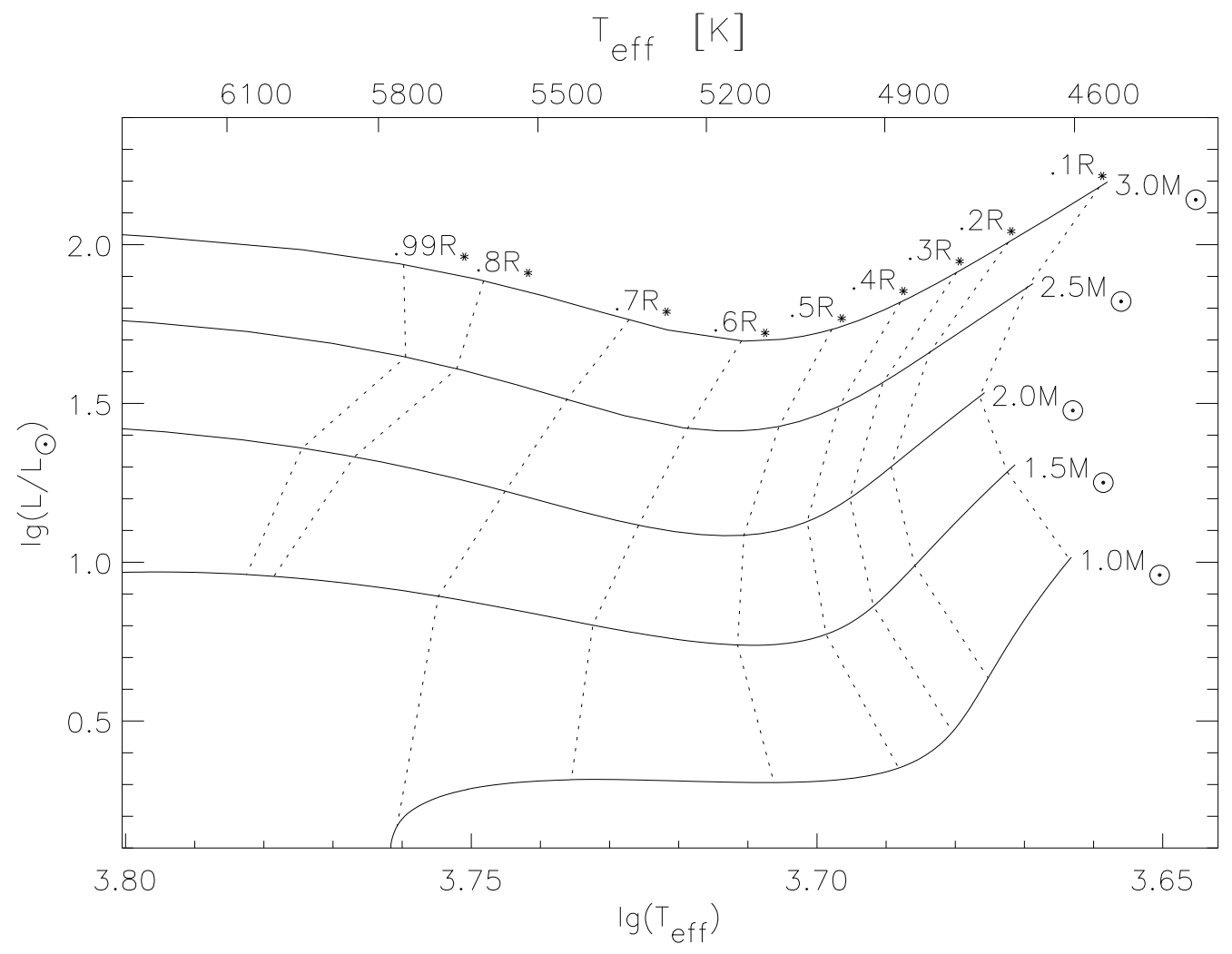

ABBILDUNG 4.2: Relative Größe $r_{\mathrm{rc}} / R_{*}$ des radiativen Kerns in Sternen während ihrer NachHauptreihen-Entwicklung. Vergleichbare Entwicklungsstadien sind mit gepunkteten Linien verbunden. 
Grenze verschiebt sich für massereichere Sterne zu niedrigeren Temperaturen. Von jeder Sequenz werden Sternmodelle ausgewählt, deren Radien des radiativen Kerns im Bereich $r_{\mathrm{rc}} \simeq 0.15 \ldots 0.35 R_{*}$ liegen, wobei $R_{*}$ der jeweilige Sternradius ist (vgl. Abb. 4.2).

\subsubsection{Die konvektive Overshoot-Region}

Die unter Verwendung der lokalen Mischungswegtheorie (MLT, mixing length theory) erzeugten Sternmodelle bestehen typischerweise aus einer schwach superadiabatischen äußeren Konvektionszone (mit $\delta \gtrsim 0$ ) oberhalb eines stark subadiabatischen radiativen Kerns (mit $\delta<0$ ). Auf dieser Grundstruktur aufbauend, wird die konvektive OvershootRegion am Übergang zwischen radiativen Kern und äußerer Konvektionszone für jedes Sternmodell individuell a posteriori bestimmt. Während bei den Geneva-ToulouseSequenzen dabei dem Ansatz von Zahn (1991) gefolgt wird und unterhalb der Konvektionszone eine polytrope Schichtung konstanter Subadiabatizität angefügt wird (vgl. Caligari 1995), wird bei den Kippenhahn-Sequenzen der Vorgehensweise von Granzer et al. (2000) gefolgt, die auf dem nicht-lokalen Mischungsweg-Ansatz von Shaviv \& Salpeter (1973) basiert: Im Gegensatz zur lokalen Beschreibung wird die radiale Bewegung eines z.B. sinkenden Gaselements in der Konvektionszone über eine Mischungslänge (etwa eine Druckskalenhöhe) hinweg verfolgt, d.h. vom Einsetzen des Absinkens bis zur Auflösung des Gaselements. Durch die nicht-lokale Beschreibung unterscheidet sich am Endpunkt der kumulative Geschwindigkeits- und Temperaturüberschuss von den lokal berechneten MLT-Werten, was zu einer geringfügigen Modifikation der Temperaturschichtung führt. Aufgrund ihrer nicht-verschwindenden Geschwindigkeit am unteren Rand $r_{\mathrm{rc}}$ der (lokal berechneten) Konvektionszone, können Gaselemente in den obersten Bereich des radiativen Kerns eindringen. Dadurch erzeugen sie eine dünne Schicht, die durch einen ins Sterninnere gerichteten konvektiven Energietransport charakterisiert wird die Overshoot-Region. Ihre untere Grenze $r_{\mathrm{bot}}\left(<r_{\mathrm{rc}}\right)$ wird durch das Verschwinden konvektiver Geschwindigkeiten definiert. Eine ausführliche Beschreibung dieses Mechanismus findet sich bei Pidatella \& Stix (1986) und Skaley \& Stix (1991). Tabelle 4.2 gibt einen Überblick über die Lage, Dicke und Subadiabatizität der Overshoot-Region in den verwendeten Sternmodellen.

\subsection{Verfahren}

Um das Ausbruchsverhalten magnetischer Flussröhre in verschiedenen Riesensternen vergleichen zu können, werden Standard-Simulationen durchgeführt, bei denen die Ausgangsbedingungen - abgesehen von denen, die sich aus der Verwendung verschiedener Sternmodelle ergeben - identisch sind. Um die Abhängigkeit der Ergebnisse von der Wahl dieser Ausgangssituation zu untersuchen, wird anschließend eine Parameterstudie durchgeführt.

Grundsätzlich wird sowohl in der linearen Stabilitätsanalyse als auch den numerischen Simulationen von einer starren Rotation des Sternkörpers ausgegangen. Unter der Voraussetzung, dass die in Riesensternen existierende differentielle Rotation nicht wesentlich größer als im Fall der Sonne ist, bewirkt sie lediglich kleine Modifikationen der 


\begin{tabular}{|c|c|c|c|c|c|c|c|}
\hline \multirow{2}{*}{$\begin{array}{c}M \\
{\left[\mathrm{M}_{\odot}\right]}\end{array}$} & \multirow[t]{2}{*}{ Nr. } & \multicolumn{2}{|c|}{$r_{\text {bot }}$} & & \multirow{2}{*}{$\begin{array}{l}\delta\left(r_{\mathrm{eq}}\right) \\
{\left[10^{-6}\right]}\end{array}$} \\
\hline & & {$\left[\mathrm{R}_{\odot}\right]$} & {$\left[\mathrm{R}_{*}\right]$} & {$\left[10^{-2} \mathrm{R}_{\odot}\right]$} & {$\left[10^{-2} \mathrm{R}_{*}\right]$} & {$\left[\mathrm{H}_{p}\right]$} & \\
\hline \multirow[t]{8}{*}{1.0} & 465 & 0.75 & 0.34 & 7.6 & 3.5 & 0.27 & -1.0 \\
\hline & 480 & 0.71 & 0.31 & 8.0 & 3.5 & 0.26 & -1.1 \\
\hline & 495 & 0.67 & 0.28 & 8.2 & 3.4 & 0.26 & -1.2 \\
\hline & 510 & 0.64 & 0.25 & 8.3 & 3.2 & 0.25 & -1.3 \\
\hline & 525 & 0.62 & 0.23 & 8.2 & 3.1 & 0.25 & -1.5 \\
\hline & 540 & 0.59 & 0.21 & 8.2 & 2.9 & 0.25 & -1.6 \\
\hline & 585 & 0.54 & 0.18 & 7.9 & 2.5 & 0.24 & -2.3 \\
\hline & 675 & 0.48 & 0.13 & 7.1 & 1.9 & 0.24 & -3.9 \\
\hline \multirow[t]{5}{*}{1.5} & 480 & 1.09 & 0.32 & 13 & 3.8 & 0.27 & -1.8 \\
\hline & 510 & 0.98 & 0.26 & 13 & 3.6 & 0.25 & -2.2 \\
\hline & 570 & 0.84 & 0.20 & 13 & 3.2 & 0.24 & -3.1 \\
\hline & 600 & 0.78 & 0.18 & 13 & 3.0 & 0.24 & -3.7 \\
\hline & 660 & 0.69 & 0.15 & 12 & 2.5 & 0.24 & -5.1 \\
\hline \multirow[t]{6}{*}{2.0} & 374 & 1.56 & 0.30 & 20 & 3.9 & 0.26 & -3.2 \\
\hline & 376 & 1.45 & 0.27 & 20 & 3.8 & 0.25 & -3.5 \\
\hline & 378 & 1.35 & 0.24 & 20 & 3.6 & 0.25 & -3.9 \\
\hline & 382 & 1.20 & 0.20 & 20 & 3.3 & 0.24 & -5.3 \\
\hline & 386 & 1.08 & 0.18 & 19 & 3.1 & 0.24 & -6.8 \\
\hline & 388 & 1.04 & 0.17 & 19 & 3.0 & 0.24 & -7.3 \\
\hline \multirow[t]{6}{*}{2.5} & 646 & 2.49 & 0.32 & 29 & 3.7 & 0.25 & -5.7 \\
\hline & 647 & 2.26 & 0.27 & 29 & 3.5 & 0.24 & -6.8 \\
\hline & 649 & 1.89 & 0.21 & 30 & 3.3 & 0.24 & -9.2 \\
\hline & 650 & 1.75 & 0.19 & 30 & 3.2 & 0.25 & -10.5 \\
\hline & 653 & 1.58 & 0.16 & 29 & 2.9 & 0.25 & -13.0 \\
\hline & 655 & 1.50 & 0.15 & 28 & 2.8 & 0.25 & -14.7 \\
\hline \multirow[t]{5}{*}{3.0} & 466 & 3.75 & 0.30 & 47 & 3.8 & 0.26 & -13.4 \\
\hline & 468 & 3.08 & 0.22 & 46 & 3.3 & 0.24 & -15.6 \\
\hline & 469 & 2.78 & 0.19 & 45 & 3.1 & 0.24 & -16.9 \\
\hline & 470 & 2.51 & 0.16 & 43 & 2.8 & 0.24 & -20.8 \\
\hline & 471 & 2.30 & 0.14 & 40 & 2.5 & 0.24 & -25.5 \\
\hline
\end{tabular}

TABELle 4.2: Lage $r_{\text {bot }}$ des unteren Rands und Ausdehnung $d_{\mathrm{os}}$ der Overshoot-Region in Riesensternen unterschiedlicher Massen und Entwicklungsstadien. Zum besseren Vergleich sind die Werte in Einheiten des Sonnenradius $\mathrm{R}_{\odot}$, Sternradius $R_{*}$ sowie der lokalen Druckskalenhöhe $H_{p}$ angegeben. Der Wert der Subadiabatizität $\delta$ gilt für die Gleichgewichtstiefe $r_{\text {eq }}$ der StandardSimulationen (Abschn. 4.2) in der Mitte der Overshoot-Region. 
Flussröhrendynamik, die im Rahmen dieser Untersuchung vernachlässigt werden können (z.B. Ferriz-Mas \& Schüssler 1995).

\subsubsection{Lineare Stabilitätsanalyse}

Zur Bestimmung der kritischen Magnetfeldstärken, die zum Einsetzen der ParkerInstabilität und der Bildung aufsteigender Flussröhrenschleifen führen, wird eine lineare Stabilitätsanalyse durchgeführt (Abschn. 2.3.2); Ausgangspunkt sind Flussröhren im mechanischen toroidalen Gleichgewicht innerhalb der Overshoot-Region (Abschn. 2.2).

Im Rahmen der Standard-Rechnungen werden die in Tab. 4.1 aufgeführten Sternmodelle untersucht, wobei von einer Rotationsrate $\Omega_{*}=\Omega_{\odot}=2.6 \cdot 10^{-6} \mathrm{~s}^{-1}$ (Sonnenrotationsrate) ausgegangen wird. Der Gleichgewichtsradius $r_{\text {eq }}$ eines Flussrings liege in der Mitte der Overshoot-Region; die Superadiabatizität $\delta\left(r_{\text {eq }}\right)$ in dieser Tiefe ist in Tab. 4.2 angegeben. Durch die Wahl des Sternmodells, der Rotationsrate und des Gleichgewichtsradius sind alle externen Größen am Ort des Flussrings festgelegt. Die Stabilitätseigenschaften und Anwachszeiten $\tau$ hängen dann nur noch von dessen Ausgangsbreite $\lambda_{\text {eq }}$ und Feldstärke $B_{\text {eq }}$ ab. Den Erkenntnissen im Fall der Sonne folgend, wird für die StandardRechnungen von der Anwachszeit $\tau=100 \mathrm{~d}$ ausgegangen. Abbildung 4.3 zeigt für jedes Sternmodell den Verlauf der $100 \mathrm{~d}$-Niveaulinie in der $(B, \lambda)$-Ebene. Vom Äquator aus ist die Magnetfeldstärke $B_{100}$ entlang einer Niveaulinie bis zu mittleren Breiten hinauf nahezu konstant. Diese Region entspricht üblicherweise der auftriebsbedingten ParkerInstabilität. $\mathrm{Zu}$ höheren Breiten hin nimmt $B_{100}$ signifikant zu: Der kleiner werdende Gleichgewichtsradius bewirkt eine stärkere Krümmungskraft, die Störungen senkrecht zur Rotationsachse stabilisiert. Bei sehr hohen Breiten führt indes die 'poleward slip'Instabilität wieder zu einer Reduktion des $B_{100}$-Wertes. Innerhalb einer Entwicklungssequenz verschieben sich die Niveaulinien typischerweise zu höheren Feldstärken. Dieses Anwachsen wird jedoch bei massereicheren Sternen immer kleiner. In dem $2.5 \mathrm{M}_{\odot}$-Stern tritt nur noch ein geringe Erhöhung des $B_{100}$-Wertes auf, während sich in der $3 \mathrm{M}_{\odot}$ Sequenz die Feldstärkeverschiebung sogar umkehrt, wodurch vergleichbare Flussringe in späteren Entwicklungsstadien bei kleineren Feldstärken instabil werden. Dieser Trend wird zusätzlich dadurch verstärkt, dass für massereichere Sterne die 100 d-Instabilität generell bei kleineren Feldstärken einsetzt.

Bis hinauf zu hohen Startbreiten dominiert die $m=1$-Instabilität. Nur nahe am Pol macht sich aufgrund der zunehmenden Krümmungskraft die 'poleward slip'-Instabilität mit der azimutalen Wellenzahl $m=0$ bemerkbar.

\subsubsection{Numerische Simulationen}

In allen Sternmodellen wird die nicht-lineare Entwicklung instabiler Flussröhren mit Startbreiten von $\lambda_{\text {eq }}=10^{\circ}, 30^{\circ}, 50^{\circ}$ und $70^{\circ}$ untersucht. Die Ausgangsfeldstärke $B_{\text {eq }}=B_{100}$ wird dem Verlauf der entsprechenden Niveaulinie in der $(B, \lambda)$-Ebene (vgl. Abb. 4.3) entnommen. Der anfängliche Röhrendurchmesser des Flussrings ist $d=$ $2000 \mathrm{~km}$. Einige Tage nach der Störung des toroidalen Gleichgewichts setzt sich der am schnellsten anwachsende Eigenmodus, hier üblicherweise der mit der Wellenzahl $m=1$, durch und führt zur Bildung einer aufsteigenden Flussröhrenschleife. 


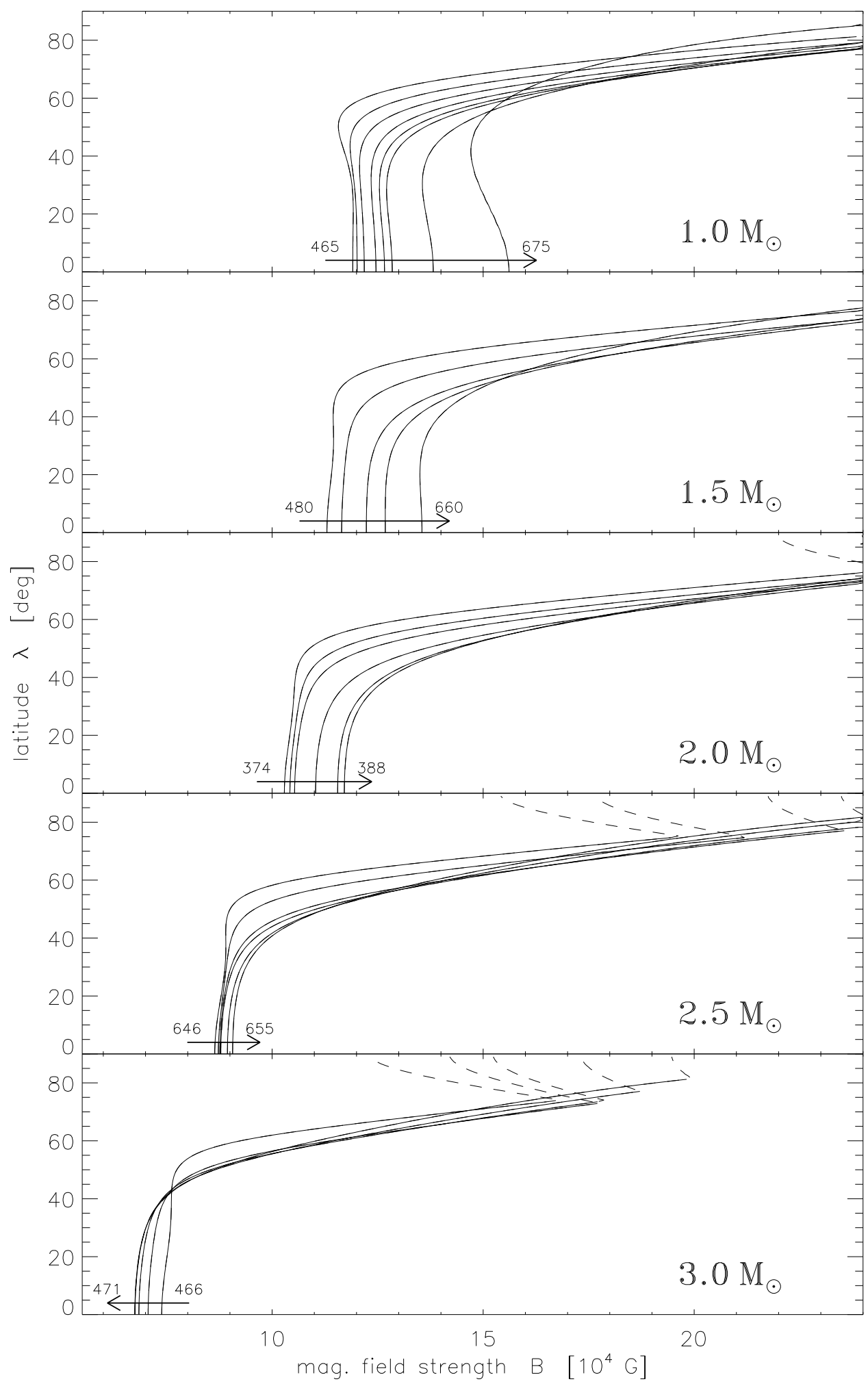

AbBILDUNG 4.3: Niveaulinien der Anwachszeit $\tau=100 \mathrm{~d}$ für Flussringe im StandardGleichgewicht (Abschn. 4.2) in der Mitte der Overshoot-Region. Es treten lediglich Instabilitäten der azimutalen Wellenzahl $m=1$ (durchgezogene Linien) und $m=0$ (gestrichelte Linien) auf. Jede Linie entspricht einem Sternmodell in Tab. 4.1, wobei die Pfeile in jeder Sequenz die chronologische Abfolge der Entwicklungsstadien anzeigen. 


\subsection{Einschluss magnetischer Flussröhren}

\subsubsection{Standardfälle}

Die Simulationen offenbaren in Riesensternen zwei grundsätzlich verschiedene Verhaltensweisen von Flussröhren, deren charakteristische Entwicklung in einem $1 \mathrm{M}_{\odot}$-Stern genauer dargestellt wird.

\section{Ausbrechende Flussröhren}

Die Abbildungen 4.4a-b illustrieren die Entwicklung einer aufsteigenden Flussröhre in einem 12.14 Gyr alten Stern (Nr. 540 in Tab. 4.2). Ihre ursprüngliche Gleichgewichtsbreite ist $10^{\circ}$. Es bildet sich eine instabile Flussröhrenschleife, die in die Konvektionszone eintritt und dort beschleunigt aufsteigt, bis sie die Oberfläche erreicht. Während des Aufstiegs sinken die in der Overshoot-Region verbleibenden Röhrensegmente ab, bis ihr zunehmender Auftrieb die radiale Komponente der Summe aus magnetischer Krümmungskraft und Corioliskraft kompensieren kann. Dieser am tiefsten im Stern liegende Teil der Flussröhre, der praktisch auf dem radiativen Kern liegt, bildet für die aufsteigende Schleife eine Art Verankerung. Durch die radiale Auftriebskraft kann allerdings die Komponente der Krümmungskraft entlang der Breite nicht ausgeglichen werden. Da diese Komponente $\mathrm{zu}$ höheren Breiten weist, rutscht der Anker auf dem radiativen Kern Richtung Pol und überquert diesen (im hier gezeigten Fall) nach etwa 1130 Tagen. Nach der Polüberquerung driftet dieser Teil, wiederum aufgrund der $\lambda$-Komponente der Krümmungskraft, Richtung Äquator. Die Entwicklung kann hier allerdings nur bis zum Zeitpunkt des Ausbruchs des Gipfelelements an der Oberfläche verfolgt werden, da die Simulation dann gestoppt werden muss.

Abbildung 4.4c zeigt die Position sowohl des tiefsten als auch höchsten Röhrenelements als Funktion der Zeit. Die azimutale Drift der aufsteigenden Flussröhrenschleife entgegen der Rotationsrichtung des Sterns spiegelt den Wellencharakter der ursächlichen Instabilität wieder. Die gesamte Entwicklung dauert (bis zum Ausbruch an der Oberfläche) etwa 3.6 Jahre.

\section{Gefangene Flussröhren}

Die Abbildungen 4.4d-f zeigen die Entwicklung einer Flussröhre in einem 12.21 Gyr alten Stern (Nr. 585). Zu Beginn ähnelt ihre Entwicklung der einer ausbrechenden Flussröhre, d.h. die aufsteigende Schleife bewegt sich beschleunigt durch die Konvektionszone nach oben, und der in der Overshoot-Region verankerte Teil der Röhre überquert den Pol des radiativen Kerns. Nach der Polüberquerung verlangsamt sich jedoch der Aufstieg des Gipfelelements derart, dass die Aufwärtsbewegung noch innerhalb der Konvektionszone, deutlich unterhalb der Oberfläche zum Stillstand kommt. Die gesamte Flussröhre bewegt sich anschließend langsam äquatorwärts, bis sie letztlich in der Äquatorebene des Sterns ein neues Gleichgewicht findet. Im Gegensatz zum ursprünglichen Ausgangsgleichgewicht umschließt die Flussröhre nun aber nicht mehr den radiativen Kern des Sterns. Die Entwicklung vom ursprünglichen, toroidalen Gleichgewicht bis zum neuen Endgleichgewicht in der Äquatorebene dauert etwa 7 Jahre. 

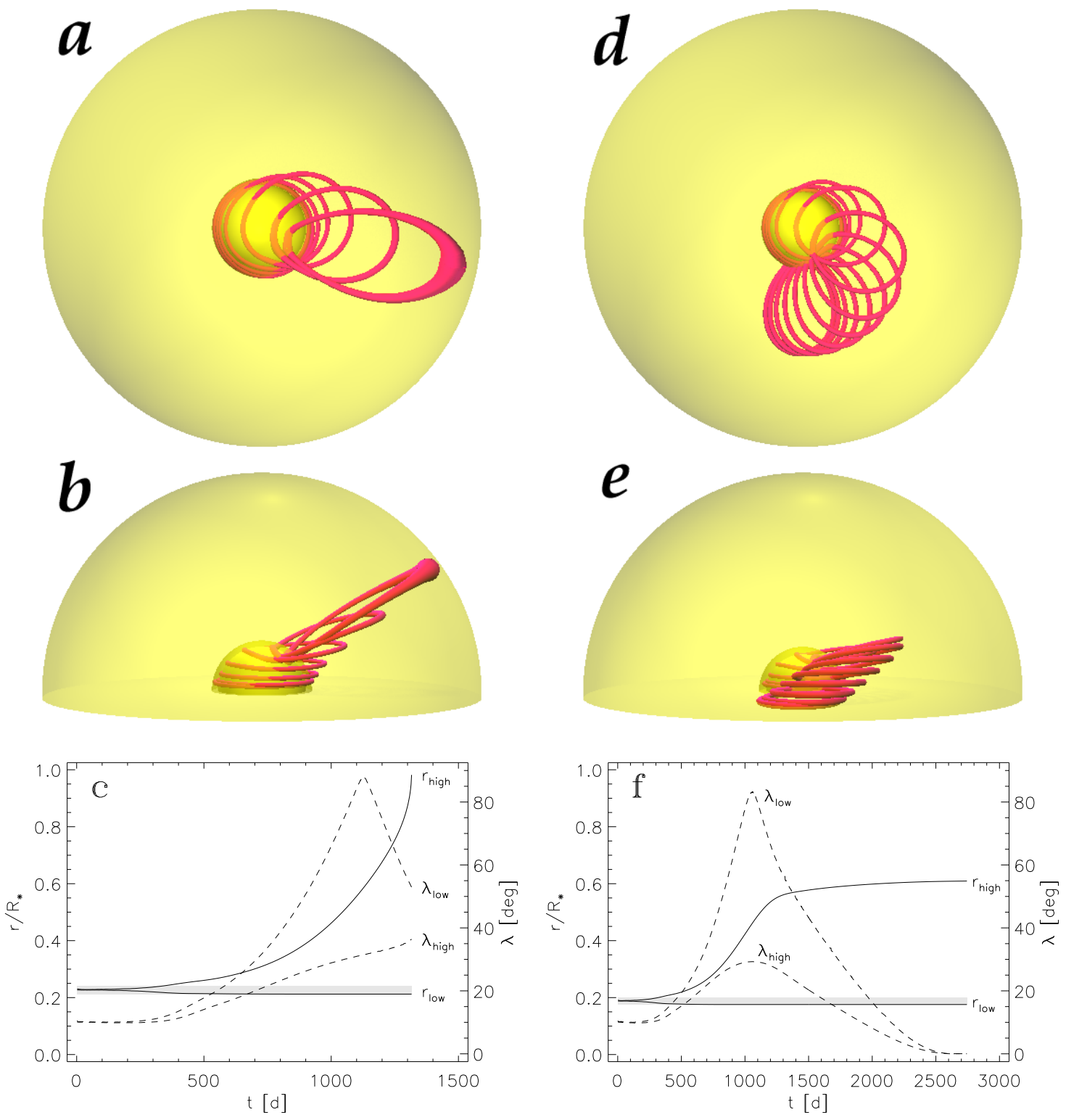

AbBILDUNG 4.4: Die Abbildungen a und b zeigen eine Kombination von Momentaufnahmen der Entwicklung einer ausbrechenden Flussröhre. Die innerste Hemisphäre ist der radiative Kern des Sterns und die (halb-transparente) dünne Schicht darüber die Overshoot-Region (Sternmodell Nr. 540, $r_{\text {bot }}=0.21 \mathrm{R}_{*}, d_{\text {os }}=2.9 \cdot 10^{-2} \mathrm{R}_{*}$ ). Die Rotation des Sterns erfolgt entgegen dem Uhrzeigersinn. Die Röhrendurchmesser wurden für die Darstellung um den Faktor 10 vergrößert. Das Diagramm c zeigt den Radius (durchgezogene Linie, linke Skala) und die Breite (gestrichelte Linie, rechte Skala) des höchsten bzw. tiefsten Flussröhrenelements im Stern als Funktion der Zeit. Das Maximum in der $\lambda_{\text {low }}$-Kurve markiert den Moment der Polüberquerung. Der grau unterlegte, horizontale Streifen entspricht der Ausdehnung der Overshoot-Region. Die Abbildungen d-f zeigen vergleichbare Darstellungen für eine gefangene Flussröhre (Sternmodell Nr. 585, $r_{\text {bot }}=0.18 \mathrm{R}_{*}, d_{\mathrm{os}}=2.5 \cdot 10^{-2} \mathrm{R}_{*}$ ). Nach der Polüberquerung kommt der Aufstieg des Gipfelelements zum Stillstand und die komplette Flussröhre driftet langsam zur Äquatorebene. 


\section{Grenzkriterium}

Die Simulationen zeigen einen Übergang von ausbrechenden zu gefangenen Flussröhren, wenn der relative Radius $\mathcal{R}$ des ursprünglichen Flussrings die Bedingung

$$
\mathcal{R}=\frac{R_{\mathrm{eq}}}{R_{*}}=\frac{r_{\mathrm{eq}}}{R_{*}} \cos \lambda_{\mathrm{eq}} \lesssim 0.17 \ldots 0.2
$$

erfüllt, wobei $R_{\text {eq }}$ der Krümmungsradius um toroidalen Gleichgewicht ist. Für größere Werte von $\mathcal{R}$ brechen Flussröhre an der Oberfläche aus, während für Werte unterhalb dieses Grenzwerts der Aufstieg gestoppt und die Flussröhre im Innern der Konvektionszone gefangen ist.

Im Verlauf der Nach-Hauptreihen-Entwicklung zum RGB wird der radiative Kern eines Sterns kleiner $\left(r_{\text {bot }} \downarrow\right)$, während sich gleichzeitig seine konvektive Hülle deutlich ausdehnt $\left(R_{*} \uparrow\right)$. Demzufolge nimmt für Flussringe bei einer festen Ausgangsbreite $\lambda_{\text {eq }}$ der relative Radius $\mathcal{R} \propto r_{\text {bot }} / R_{*}$ mit zunehmendem Sternalter ab (vgl. Gl. [4.1] mit $\left.r_{\text {eq }} \sim r_{\text {bot }}\right)$. Wegen $\mathcal{R} \propto \cos \lambda_{\text {eq }}$ werden Flussröhren bei hohen Breiten zuerst eingeschlossen, während die bei niederen Breiten startenden immer noch ausbrechen. Als Beispiel zeigt Abb. 4.5 die radiale Entwicklung des Gipfelelements im Sternmodell Nr. 540 in Abhängigkeit von der Ausgangsbreite $\lambda_{\text {eq }}$ des Flussrings: Während Flussröhren mit $\lambda_{\text {eq }} \lesssim 33^{\circ}$ ausbrechen, sind die bei höheren Anfangsbreiten gefangen. Erst wenn $\mathcal{R}$ für alle Breiten unter den kritischen Wert (4.1) fällt, ist der Ausbruch magnetischer Flussröhren vollständig unterbunden.

Der Übergang von ausbrechenden zu gefangenen Flussröhren tritt in allen untersuchten Entwicklungssequenzen auf. Abbildung 4.6 zeigt ein theoretisches HR Diagramm, in dem jedes untersuchte Sternmodelle entsprechend des Ausbruchsverhaltens seiner Flussröhren mit einem anderen Symbole markiert ist.

\subsubsection{Parameterstudie}

Die Standard-Simulationen in Abschn. 4.3.1 wurden alle unter den selben Ausgangsbedingungen durchgeführt: Sonnenrotationsrate, 100 Tage Anwachszeit der linearen Instabilität, $2000 \mathrm{~km}$ Anfangsdurchmesser der Flussröhre und Gleichgewichtsposition in der Mitte der Overshoot-Region. Um die Abhängigkeit der Ergebnisse von diesen speziellen Annahmen zu untersuchen, wird nun sukzessive ein Parameter innerhalb angemessener Grenzen variiert, während die anderen unverändert bleiben.

\section{Rotationsrate}

Aufgrund ihrer magnetischen Abbremsung und wachsenden Ausdehnung verlangsamt sich die Rotation von Einzelsternen während ihrer Nach-Hauptreihen-Entwicklung gravierend. Andererseits kann die Rotationsrate von Riesensternen in gezeitengebundenen engen Doppelsternsystemen (z.B. RS CVn-Systemen) die der Sonne auch deutlich übertreffen. Aus diesem Grund wird die Rotationsrate im Bereich $\Omega_{*}=0.1 \ldots 10 \Omega_{\odot}$ variiert.

Grundsätzlich ist festzustellen, dass die Rotationsrate keinen signifikanten Einfluss auf das Ausbruchsverhalten von Flussröhre im Vergleich zu den Standard-Simulationen 


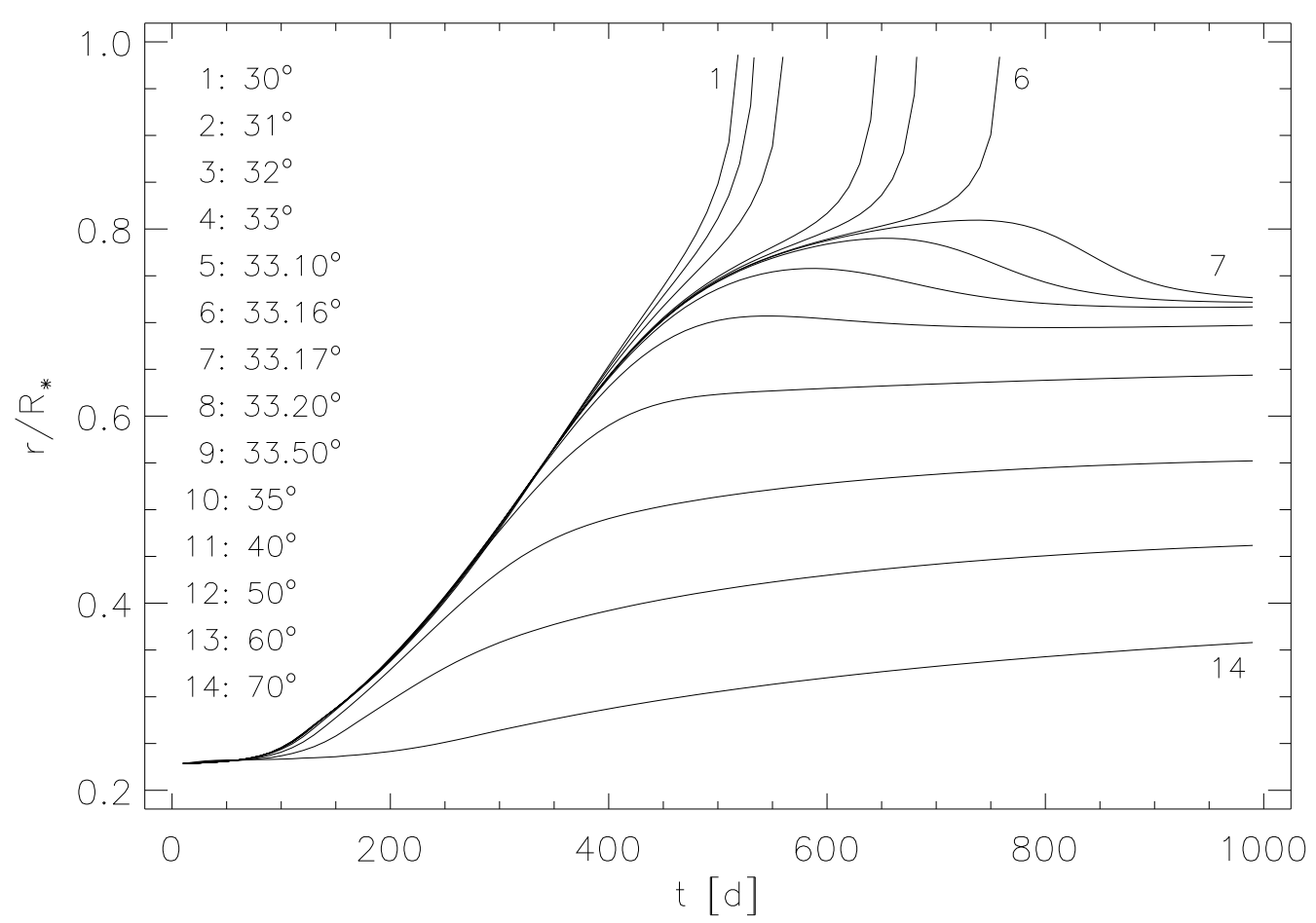

AbBILDUNG 4.5: Radiale Entwicklung des höchsten Röhrenelements im $1 \mathrm{M}_{\odot}$-Stern Nr. 540 als Funktion der Ausgangsbreite $\lambda_{\text {eq }}$. Der Übergang zwischen ausbrechenden und gefangenen Flussröhren findet bei $\lambda_{\text {eq }} \simeq 33.16^{\circ}$ statt. Die Ausgangsfeldstärke ist einheitlich $B=2 \cdot 10^{5} \mathrm{G}$, damit auch Flussröhren bei hohen Breiten in den Vergleich mit einbezogen werden können.

hat. Allerdings zeigt sich, dass Flussröhren bei hohen Rotationsraten dazu neigen, bei hohen Breiten zu verharren und nicht zum Äquator abzusinken. Sie befinden sich dabei in einem dynamischen Zustand, in dem sowohl der in der Overshoot-Region (bei hohen Breiten) verankerte Teil als auch der Gipfel der Flussröhrenschleife um die Rotationsachse des Sterns präzedieren. Diese Flussröhren brechen nicht aus und werden daher als 'gefangen' eingestuft, obwohl sie das in den Standard-Rechnungen eingenommene, äquatoriale Gleichgewicht nicht erreichen. Für kleine Rotationsraten verschiebt sich die Übergangsbreite, die ausbrechende von gefangenen Flussröhren trennt, leicht zu höheren Breiten.

\section{Anwachszeit bzw. Anfangsfeldstärke}

Die lineare Stabilitätsanalyse ergibt, dass die kritische Feldstärke, ab der Instabilität einsetzt, nur etwa $15 \%$ unterhalb der $B_{100}$-Feldstärke liegt. Längere Anwachszeiten $\tau$, die näher an der Instabilitätsgrenze liegen, führen daher nur zu einer unbedeutenden Verringerung der Ausgangsfeldstärke und geringen Modifikation des StandardGleichgewichts. Andererseits entspricht eine Verringerung von $\tau$ einem signifikanten Anstieg der Feldstärke $B_{\tau}$. Es wurden daher zusätzliche Simulationen für Anwachszeiten bis hinunter zu $\tau=16 \mathrm{~d}$ durchgeführt, was typischerweise Feldstärken entspricht, die etwa doppelt so hoch sind wie die in den Standardfällen. 


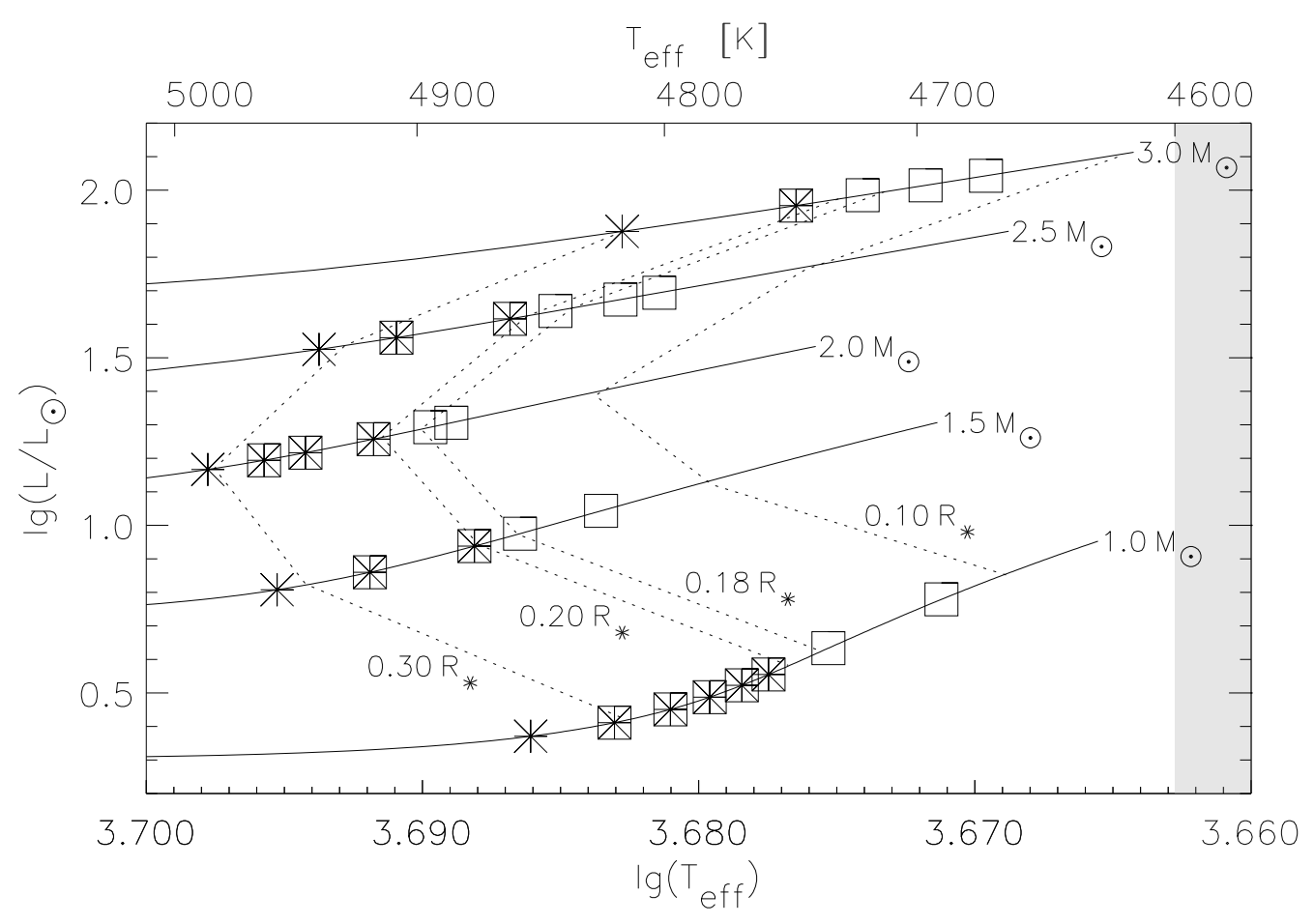

ABBILDUNG 4.6: Änderung des Ausbruchsverhalten magnetischer Flussröhren am Fuße des RGB. Gepunktete Linien verbinden vergleichbare Entwicklungsstadien (in Bezug auf die relative Größe des radiativen Kerns). Kreuze bzw. Quadrate markieren Sternmodelle mit ausbrechenden bzw. gefangenen Flussröhren, beide Symbole wurden übereinander gezeichnet, wenn Flussröhren mit hohen Startbreiten gefangen sind, bei niederen hingegen noch ausbrechen. Die grau unterlegte Zone am rechten Rand markiert den Bereich, in dem verschiedene Autoren die Koronagrenze beobachten.

Höhere Feldstärken führen zu einer drastischen Beschleunigung der zeitlichen Entwicklung sowohl ausbrechender als auch gefangener Flussröhren, ändern aber nichts an deren individuellen Endzustand; insbesondere wird die Übergangsbreite nur unwesentlich beeinflusst.

\section{Magnetischer Fluss bzw. Röhrendurchmesser}

Der magnetische Fluss $\Phi$ einer FR bestimmt sich aus ihrer Querschnittsfläche $(\propto d)$ und der Feldstärke $B$. Mit der Annahme eines anfänglichen Röhrendurchmessers von $d=$ $2000 \mathrm{~km}$ und Feldstärken $B_{100}$ entsprechend der linearen Stabilitätsanalyse (Abb. 4.3), überdecken die Standard-Simulationen einen Bereich $\Phi \sim 10^{21} \ldots 10^{22} \mathrm{Mx}$, der mit magnetischen Flüssen großer aktiven Regionen auf der Sonne vergleichbar ist. Wenngleich Sternflecke auf aktiven Riesensternen wesentlich größer sind (z.B. Strassmeier 1999), können Beobachtungen nicht zwischen den Ausbrüchen weniger großer Flussröhren oder sehr vielen kleinen unterscheiden. Da die Overshoot-Region von Riesensternen sehr ausgedehnt ist (Tab. 4.2), würden weder wenige große noch eine große Anzahl von kleinen Flussröhren der Modellannahme von isolierten Flussröhren widersprechen. Die Abmes- 
sungen einer Flussröhre wirken sich auf ihren aerodynamischen Strömungswiderstand aus, dessen relativer Einfluss mit wachsendem Röhrendurchmesser kleiner wird.

Um dies zu untersuchen wurden Simulationen mit der Standard-Feldstärke $B_{100}$, aber unterschiedlichen (anfänglichen) Röhrendurchmessern von $d=1000 \mathrm{~km}$ bzw. $4000 \mathrm{~km}$ durchgeführt. Die Resultate zeigen, dass dieser Bereich von $d$ bereits in dem Regime liegt, in dem die Wechselwirkung mit der Umgebung keinen Einfluss auf den Endzustand der Entwicklung hat.

\section{Gleichgewichtstiefe}

Die Stabilitätseigenschaften von Flussringen hängen empfindlich vom Wert der Superadiabatizität $\delta\left(r_{\text {eq }}\right)$ ab, der im Bereich der Overshoot-Region um etwa einen Faktor 100 variiert. Aus diesem Grund wurden zusätzliche Rechnung durchgeführt, bei denen die Gleichgewichtsposition $r_{\text {eq }}$ am oberen Rand der Overshoot-Region bzw. $2000 \mathrm{~km}$ über ihrem unteren Rand liegt.

Bei der tieferen Startposition sind aufgrund der stabileren Schichtung wesentlich höhere Feldstärken erforderlich, um das Einsetzen der Instabilität zu erreichen. Daraus folgt wiederum die oben beschriebene Beschleunigung der Flussröhrenentwicklung. Die Endstadien unterscheiden sich dabei kaum von denen der Standard-Simulationen. Das gleiche gilt i.Allg. auch für Flussröhren, die vom oberen Rand der Overshoot-Region starten. Allerdings treten hier einige Ausnahmefälle auf, die ein modifiziertes Ausbruchsverhalten zeigen: Flussringe, die von sehr hohen Breiten starten, lösen sich vollständig von der Overshoot-Region ab und driften entlang der Rotationsachse zur Oberfläche, bis sie in der Umgebung des Pols ausbrechen. Ein ähnliches Verhalten wurde auch bei Flussröhren in schnell rotierenden T-Tauri Sternen gefunden (Granzer et al. 2000).

\section{Struktur der Overshoot-Region}

Die Struktur der Overshoot-Region am Übergang zwischen äußerer Konvektionszone und radiativen Kern ist noch nicht vollständig verstanden und Gegenstand aktueller Forschung. Im Fall der Sonne ergibt die Analyse helioseismischer Daten (z.B. Basu 1997) eine Ausdehnung der Overshoot-Region, die deutlich kleiner ist als sie von theoretischen Modellen prognostiziert wird. Allerdings geht man bei dieser Analyse von der (vermutlich sehr unrealistischen Annahme) einer idealisierten sphärisch-symmetrischen Schicht aus. Die wichtigsten Eigenschaften der Overshoot-Region in Bezug auf das Flussröhrenmodell sind ihre Ausdehnung und Superadiabatizität: In der Sonne muss diese Schicht ausreichend dick sein, um die Speicherung des durch Beobachtungen über einen Aktivitätszyklus hinweg ermittelten magnetischen Gesamtfluss zu ermöglichen. Der Wert der Subadiabatizität bestimmt indes den kritischen Feldstärkebereich der Parker-Instabilität. Im vorliegenden Fall wurden zusätzliche Flussröhrensimulationen in Sternmodellen durchgeführt, die mit dem Geneva-Toulouse-Sternentwicklungsprogamm (z.B. Charbonnel \& Talon 1999) erzeugt wurden (vgl. Abb. 4.1). Die Overshoot-Region wurde dabei entsprechend dem Ansatz von Zahn (1991) durch eine polytrope Schicht konstanter Subadiabatizität modelliert; für eine ausführliche Beschreibung dieses Verfahrens wird auf Caligari (1995) verwiesen. Die Entwicklung und der Einschluss magnetischer Flussröhren 
in diesen zusätzlichen Sternmodellen führt zu den gleichen Ergebnissen wie in den oben dargestellten Standardfällen (Schüssler et al. 2001).

Zusammenfassend lässt sich sagen, dass die Variation der Parameter nur einen geringfügigen Einfluss (wenn überhaupt) auf das Schicksal aufsteigender Flussröhren hat. Die Entscheidung, ob eine Flussröhre an der Oberfläche ausbricht oder im Innern der Konvektionszone eingeschlossen wird, hängt praktisch ausschließlich von ihrem anfänglichen relativen Krümmungsradius $\mathcal{R}$ ab. 


\section{Kapitel 5}

\section{Einschlussmechanismus}

\subsection{Kräfteanalyse}

Um den Grund für das unterschiedliche Ausbruchsverhalten in Riesensternen zu bestimmen, werden eine ausbrechende Flussröhre, die im $1 \mathrm{M}_{\odot}$-Stern (Nr. 540, vgl. Abb. 4.4ac) bei einer Ausgangsbreite von $\lambda_{\text {eq }}=10^{\circ}$ startet, und eine gefangene Flussröhre, die im selben Sternmodell bei $40^{\circ}$ startet, miteinander verglichen. Beide Flussröhren beginnen ihre Entwicklung in der Mitte der Overshoot-Region mit der gleichen Feldstärke. Sie unterscheiden sich lediglich in ihrer Startbreite, d.h. ihrem Krümmungsradius, und ihrer internen Strömungsgeschwindigkeit, die notwendig ist um die Krümmungskraft im Gleichgewicht zu kompensieren. Der Übergang zwischen ausbrechenden und gefangenen Röhren liegt hier bei etwa $29.5^{\circ}$ Breite. Abbildung 5.1 zeigt die jeweiligen Beiträge der beteiligten Kräfte zur radialen Beschleunigung des Gipfelelements der Flussröhre in Abhängigkeit von dessen Abstand zum Sternmittelpunkt.

Der Strömungswiderstand der Flussröhre hemmt grundsätzlich den Aufstieg des Gipfelelements und spiegelt dabei dessen Geschwindigkeit wider. Da er eine Konsequenz der Bewegung und nicht ihre Ursache ist, kann er die Entwicklung lediglich modifizieren, den Endzustand aber nicht nachhaltig verändern. Aus diesem Grund ist dieser Beitrag hier nur von untergeordnetem Interesse. Die Beschleunigung aufgrund von Trägheitskräften ist hauptsächlich auf die Corioliskraft zurückzuführen. Ihr relativer Beitrag zum Aufstieg des Gipfelelements ist sowohl in der ausbrechenden als auch der gefangenen Flussröhre (im Vergleich zur Krümmungskraft) von vergleichbarer Größe, weshalb anzunehmen ist, dass sie ebenfalls keinen entscheidenden Einfluss auf den Endzustand hat. Der Auftrieb einer Flussröhre ist die treibende Kraft einer aufsteigenden Schleife. Bei einer gefangenen Flussröhre ist er im unteren Teil der Konvektionszone etwas stärker als bei einer ausbrechenden, nimmt im Vergleich zu dieser aber mit der Höhe etwas langsamer zu. Etwa in der Mitte der Konvektionszone fällt der Beitrag des Auftriebs bei der gefangenen Flussröhre unter den entsprechenden Wert der ausbrechenden. Das Anwachsen der nach innen gerichteten magnetischen Krümmungskraft erklärt sich grundsätzlich durch die größer werdende Krümmung der aufsteigenden Schleife, die den Rückgang des Magnetfelds in diesem Segment (aufgrund der adiabatischen Expansion während des Aufstiegs) überkompensiert.

Diese Zusammenhänge weisen darauf hin, dass das spezifische Verhalten sowohl der 


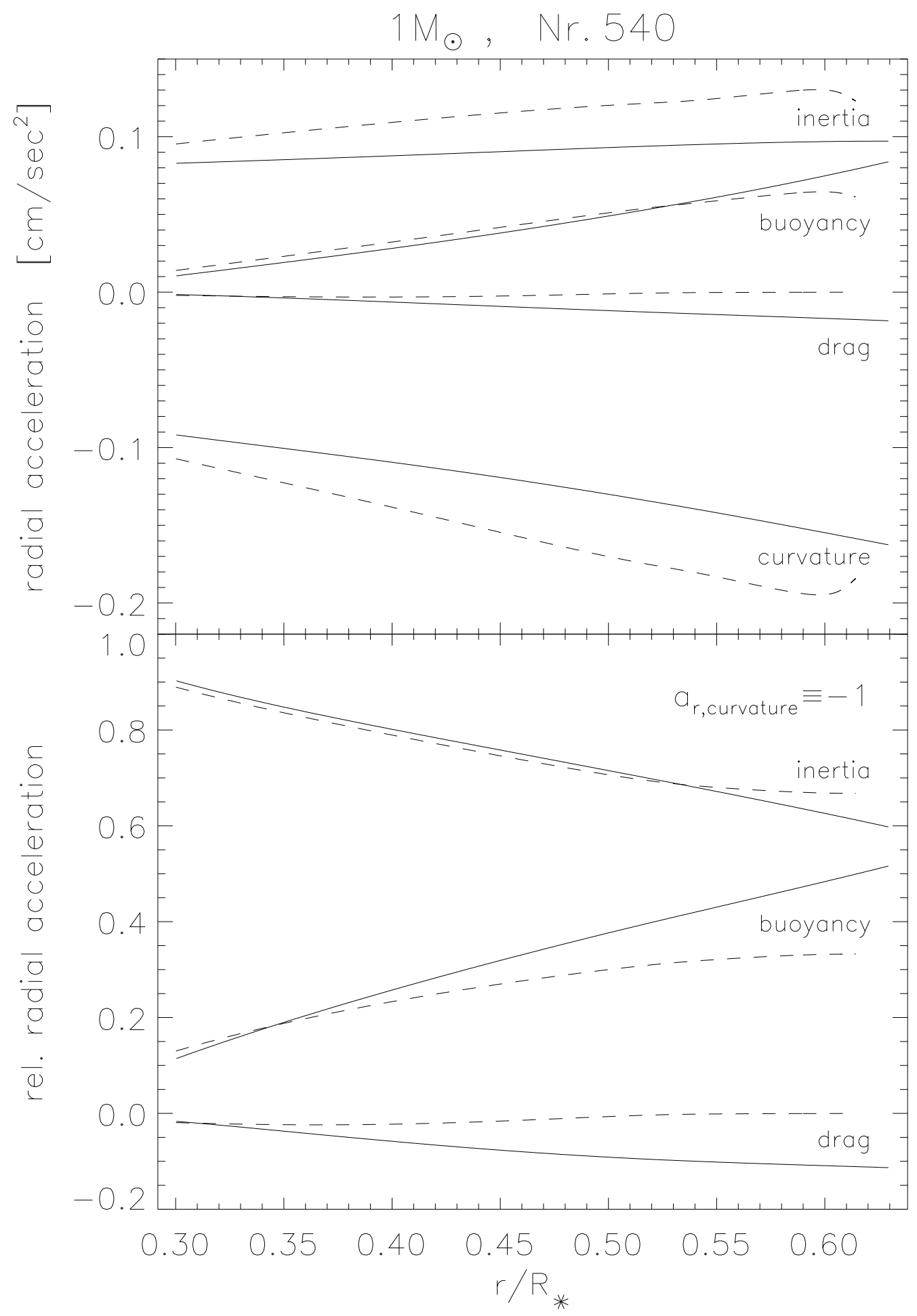

ABBILDUNG 5.1: Vergleich der Beiträge verschiedener Kräfte zur radialen Beschleunigung des Gipfelelements einer ausbrechenden (durchgezogene Linien) und einer gefangenen Flussröhre (gestrichelte Linien). Das untere Diagramm zeigt die relativen Beiträge in Bezug auf die magnetische Krümmungskraft, die auf -1 gesetzt wurde. Die Ausgangsfeldstärke beider Flussröhren ist $B=1.28 \cdot 10^{5} \mathrm{G}$. 
Auftriebs- als auch der Krümmungskraft für den Einschluss magnetischer Flussröhren in Riesensternen verantwortlich sind. Der langsamere Anstieg des Auftriebs mit der Höhe in einer gefangenen Schleife erklärt sich durch eine geringere Abströmung von Materie aus dem Gipfelsegment im Vergleich zu ausbrechenden Flussröhren. Bei der Krümmungskraft wird dagegen der Rückgang des Magnetfelds durch die starke Zunahme der Krümmung mehr als ausgeglichen. In gefangenen Flussröhren wird der Rückgang der nach außen gerichteten Auftriebskraft von einer stetig anwachsenden, nach innen gerichteten Krümmungskraft begleitet, wodurch letztlich eine Abbremsung der radialen Bewegung des obersten Flussröhrensegments hervorgerufen wird. Flussringe bei höheren Breiten beginnen ihre Entwicklung bereits mit einer stärkeren Krümmung und sind gegenüber diesem abbremsenden Effekt anfälliger. Oberhalb einer kritischen Gleichgewichtsbreite (der Übergangsbreite) dominiert dieser Effekt die Entwicklung des Gipfelelements und beendet ihren Aufstieg noch innerhalb der Konvektionszone.

\subsection{Modell einer polytropen Schichtung}

Die wesentlichen Zusammenhänge, die zum Einschluss magnetischer Flussröhren in Doppelsternen führen, werden anhand des vereinfachten Modells eines polytropen Sterns dargestellt, in dem der charakteristische Mechanismus des ursprünglichen Problems leichter nachvollzogen werden kann.

Ausgangspunkt ist eine hydrostatische Schichtung (ohne Rotation). Unter der Annahme eines Potenzgesetzes für die Gravitationsbeschleunigung wird die Schichtung durch die Gleichgewichtsbedingung

$$
\frac{d p}{d r}=-\rho g=-\rho(r) g_{0}\left(\frac{r_{0}}{r}\right)^{\sigma}
$$

beschrieben. Der Index ‘0’ bezeichnet Größen am Referenzradius $r_{0}$, der Exponent $\sigma$ (> 0 ) bestimmt die Abnahme der Gravitation mit zunehmendem Radius $r$. Das Verhältnis zwischen dem Gasdruck $p$ und der Dichte $\rho$ wird durch die Polytropenbeziehung

$$
p=K \rho^{\gamma^{*}}
$$

bestimmt, wobei $K$ die Konstant und $\gamma^{*}$ der Exponent der Polytrope ist. Aus der Kombination der Gln. (5.1) und (5.2) ergibt sich die Form

$$
-g_{0}\left(\frac{r_{0}}{r}\right)^{\sigma} \mathrm{d} r=\gamma^{*} K \rho^{\left(\gamma^{*}-2\right)} \mathrm{d} \rho,
$$

die vom Referenzradius $r_{0}$ bis $r$ aufintegriert wird. Mit der üblichen Definition des logarithmischen Temperaturgradienten

$$
\nabla^{*}=\left(\frac{d \ln T}{d \ln p}\right)=\frac{\gamma^{*}-1}{\gamma^{*}}
$$

und der Abkürzung

$$
\eta(r)=1-\frac{1}{\sigma-1} \nabla^{*} \frac{r_{0}}{H_{\mathrm{p} 0}}\left(1-\left(\frac{r_{0}}{r}\right)^{\sigma-1}\right),
$$




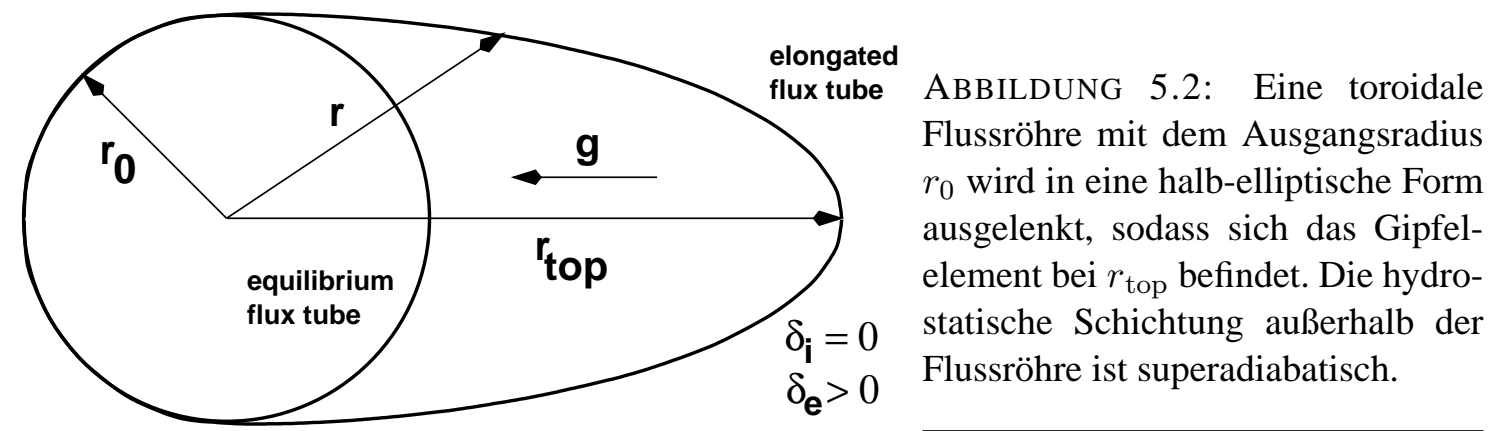

wobei $H_{p 0}=p_{0} /\left(\rho_{0} g_{0}\right)$ die Druckskalenhöhe am Referenzradius $r_{0}$ ist, lassen sich die Lösungen für die Dichte- und Druckschichtung explizit angeben:

$$
\begin{aligned}
& \rho(r)=\rho_{0} \eta(r)^{1 / \nabla^{*}-1} \\
& p(r)=p_{0} \eta(r)^{1 / \nabla^{*}}
\end{aligned}
$$

Die obere Grenze $R_{\mathrm{pl}}$ der polytropen Schicht wird durch die Bedingung $\eta_{\mathrm{e}}\left(R_{\mathrm{pl}}\right)=0$ festgelegt.

Die weiteren Überlegungen gehen von einer toroidalen Flussröhre der Feldstärke $B_{0}$ aus, die in der Äquatorebene des polytropen Sterns bei $r=r_{0}$ liegt. Der Flussring befinde sich im mechanischen Gleichgewicht, d.h die magnetische Krümmungskraft wird durch die Auftriebskraft kompensiert:

$$
\frac{B_{0}^{2}}{4 \pi r_{0}}=\left(\rho_{\mathrm{e} 0}-\rho_{0}\right) g_{0}
$$

Dabei sind $\rho_{\mathrm{e} 0}$ und $\rho_{0}$ die externe bzw. interne Dichte am Gleichgewichtsradius $r_{0}$. Die entsprechenden Gasdrücke werden durch die laterale Druckgleichgewichtsbedingung miteinander verknüpft:

$$
p_{\mathrm{e} 0}=p_{0}+\frac{B_{0}^{2}}{8 \pi}=p_{0}\left(1+\frac{1}{\beta_{0}}\right)
$$

Dabei ist $\beta_{0}=8 \pi p_{0} / B_{0}^{2}$. Um die Entwicklung einer langsam aufsteigenden Flussröhrenschleife nachzubilden, wird der Flussring in eine halb-elliptische Konfiguration verformt (vgl. Abb. 5.2). Bei einer quasi-statischen, adiabatischen Auslenkung wird die Schichtung im Innern der gestreckten Flussröhre durch die Gln. (5.6) und (5.7) mit $\nabla^{*}=\nabla_{\text {ad }}$ beschrieben. Die externe Konvektionszone ist hingegen superadiabatisch geschichtet: $\nabla^{*}{ }_{\mathrm{e}}=\nabla_{\mathrm{ad}}+\delta_{\mathrm{e}}\left(\operatorname{mit} \delta_{\mathrm{e}}>0\right)$. Ein quasi-statischer Aufstieg ist eine gute Näherung für die numerischen Simulationen, außer für die oberste, stark superadiabtische Schicht der Konvektionszone. Die Dichte $\rho_{0}$ am Referenzniveau $r_{0}$ muss bei diesem Vorgehen für jede Auslenkung iterativ angepasst werden, um die Massenerhaltung im Innern der Flussröhre zu gewährleisten. Um festzustellen, ob die Flussröhrenschleife gefangen ist, wird das Verhältnis zwischen der nach außen gerichteten Auftriebskraft $a_{\text {out }}$ und der nach innen gerichteten Krümmungskraft $a_{\text {in }}$ an ihrem Gipfelpunkt als Funktion der Höhe $r_{\text {top }}$ untersucht. Abbildung 5.3 gibt den Verlauf dieses Verhältnisses in der $\left(r_{0}, r_{\text {top }}\right)$-Ebene wieder. Die Zahlenwerte wurden dabei so gewählt, dass sie den Bedingungen im $1 \mathrm{M}_{\odot}$-Stern Nr. 540 entsprechen: $\rho_{\mathrm{e} 0}=0.4 \mathrm{~g} / \mathrm{cm}^{3}, p_{\mathrm{e} 0}=2.1 \cdot 10^{14} \mathrm{dyn} / \mathrm{cm}^{2}, g_{0}=2.3 \cdot 10^{3} \mathrm{~cm} / \mathrm{s}^{2}$ 
und $\beta_{0}=10^{6}$. Um den signifikanten Teil der Sternmasse $\left(M\left(r_{0}\right) / M_{*} \simeq 0.34\right)$ innerhalb der Konvektionszone $\left(r>r_{0}\right)$ zu berücksichtigen, wurde der Gravitationsexponent $\sigma=1.3$ verwendet. Der adiabatische Temperaturgradient ist $\nabla_{\mathrm{ad}}=0.4$ und die Superadiabatizität $\delta_{\mathrm{e}}=10^{-6}$. Der Gleichgewichtsradius variiert um $r_{0} \approx 4.28 \cdot 10^{10} \mathrm{~cm}$. Für $r_{0} / R_{\mathrm{pl}} \lesssim 0.183$ zeigt Abb. 5.3 das Auftreten eines Bereichs (grau unterlegt), in dem die Krümmungskraft stärker als die Auftriebskraft ist. Dieser Bereich wird von zwei Gleichgewichtszuständen begrenzt, einem stabilen bei größeren Radien und einem instabilen bei kleineren. Flussröhren, die den unteren Gleichgewichtszustand erreichen, sind gefangen.

Dieses Ergebnis wird mit einer entsprechenden Analyse der nicht-linearen Simulationen verglichen. Abbildung 5.4 zeigt das Verhältnis zwischen den nach außen bzw. innen gerichteten Kräfte

$$
\left|\frac{f_{\text {out }}}{f_{\text {in }}}\right|=\left|\frac{f_{\text {buoy }}+f_{\text {inertia }}}{f_{\text {curv }}+f_{\text {drag }}}\right|
$$

am Gipfelpunkt aufsteigender Flussröhrenschleifen im $1 \mathrm{M}_{\odot}$-Stern Nr. 540, die zwischen den Gleichgewichtsbreiten $\lambda_{\mathrm{eq}}=25 \ldots 30^{\circ}$ starten. Auch bei den nicht-linearen Simulationen zeigt sich ein Bereich, in dem die Summe der nach innen gerichteten Kräfte dominiert $^{1}$. Flussröhren, die ihre Entwicklung etwas unterhalb der Übergangsbreite beginnen, erfahren in der Mitte der Konvektionszone eine vorübergehende Abbremsung ihres Aufstiegs. Aufgrund ihres Trägheitsmoments können sie diese Region jedoch durchqueren ohne eingeschlossen zu werden. Je kleiner aber der Krümmungsradius $\mathcal{R}$ des Flussrings im Gleichgewicht ist, desto breiter ist diese abbremsende Zone und dementsprechend größer die Wahrscheinlichkeit für eine Flussröhre gefangen zu werden. Flussröhren, die oberhalb der Übergangsbreite starten, sind nicht mehr in der Lage die abbremsende Zone zu durchqueren: Typischerweise steigt das Gipfelelement etwas über den designierten Gleichgewichtsradius hinaus auf, bis es von der rücktreibenden Krümmungskraft zurückgezogen wird; dieser Vorgang ist auch in Abb. 4.5 bei Flussröhren zu erkennen, die etwas oberhalb der Übergangsbreite starten. Bei noch höheren Anfangsbreiten ist die Beschleunigung bei kleinen Auslenkungen bereits so gering, dass sich Trägheitseffekte nicht mehr bemerkbar machen und vernachlässigt werden können; das oberste Flussröhrensegment nähert sich in diesen Fällen asymptotisch der neuen Gleichgewichtshöhe.

Aufgrund der sehr guten Übereinstimmung der Ergebnisse des einfachen (quasistatischen) Modells mit denen der nicht-linearen Simulationen ist davon auszugehen, dass der Einschlussmechanismus magnetischer Flussröhren in Riesenstern tatsächlich durch das Wechselspiel zwischen magnetischer Krümmungskraft und Auftriebskraft verursacht wird.

\footnotetext{
${ }^{1}$ Die sehr gute (qualitative) Übereinstimmung vergleichbarer Strukturen in den beiden Abbn. 5.3 und 5.4 bestätigt die Analogie zwischen dem vereinfachten und dem vollständigen Flussröhrenmodell.
} 


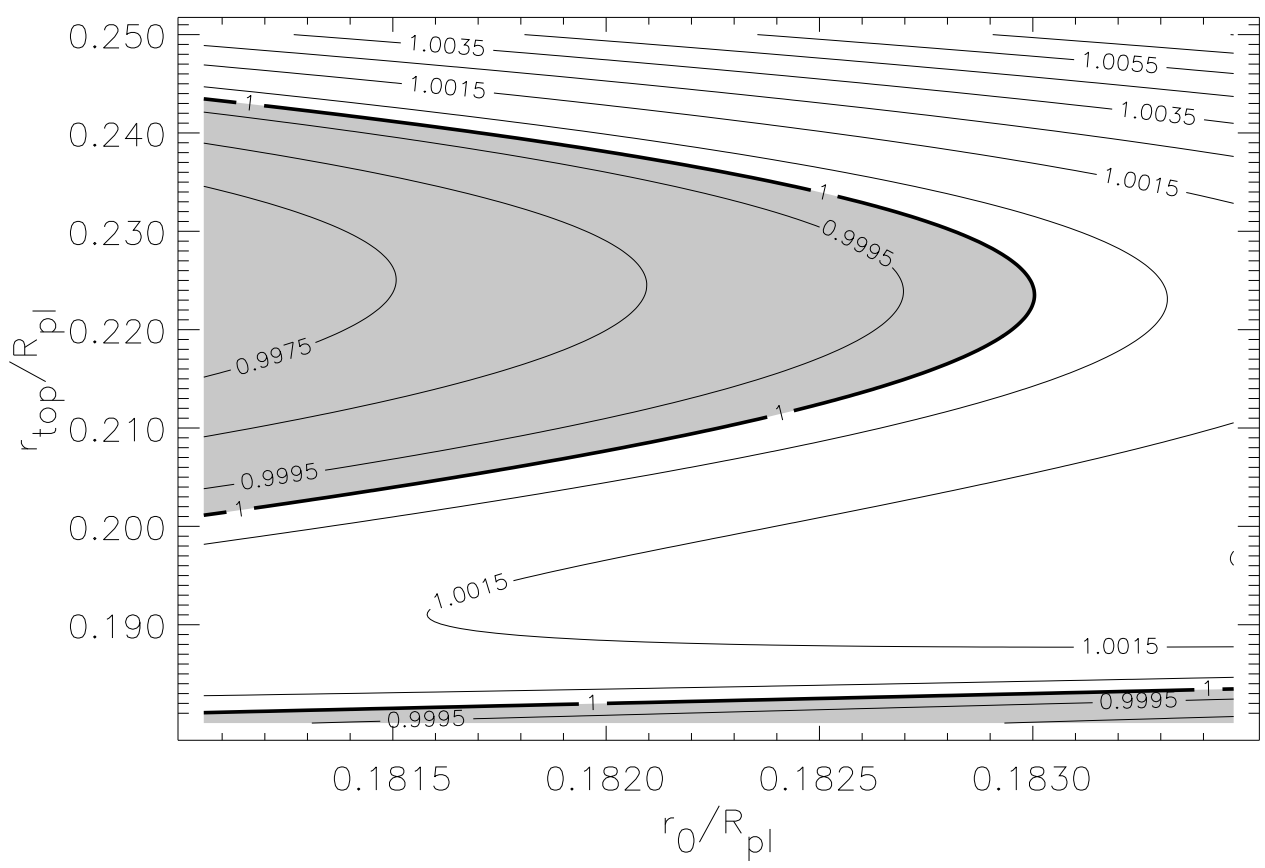

AbBILDung 5.3: Polytrope Schichtung: Verhältnis $\left|a_{\text {out }} / a_{\text {in }}\right|$ am Gipfelelement $r_{\text {top }}$ der halbelliptisch ausgelenkten Flussröhre. Der Bereich, in dem die magnetische Krümmungskraft die Auftriebskraft übertrifft ist grau unterlegt; dicke Linien markieren (statische) Gleichgewichtszustände.

$$
\lambda_{\text {eq }} \text { [degrees] }
$$

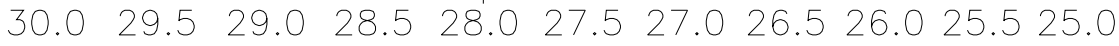

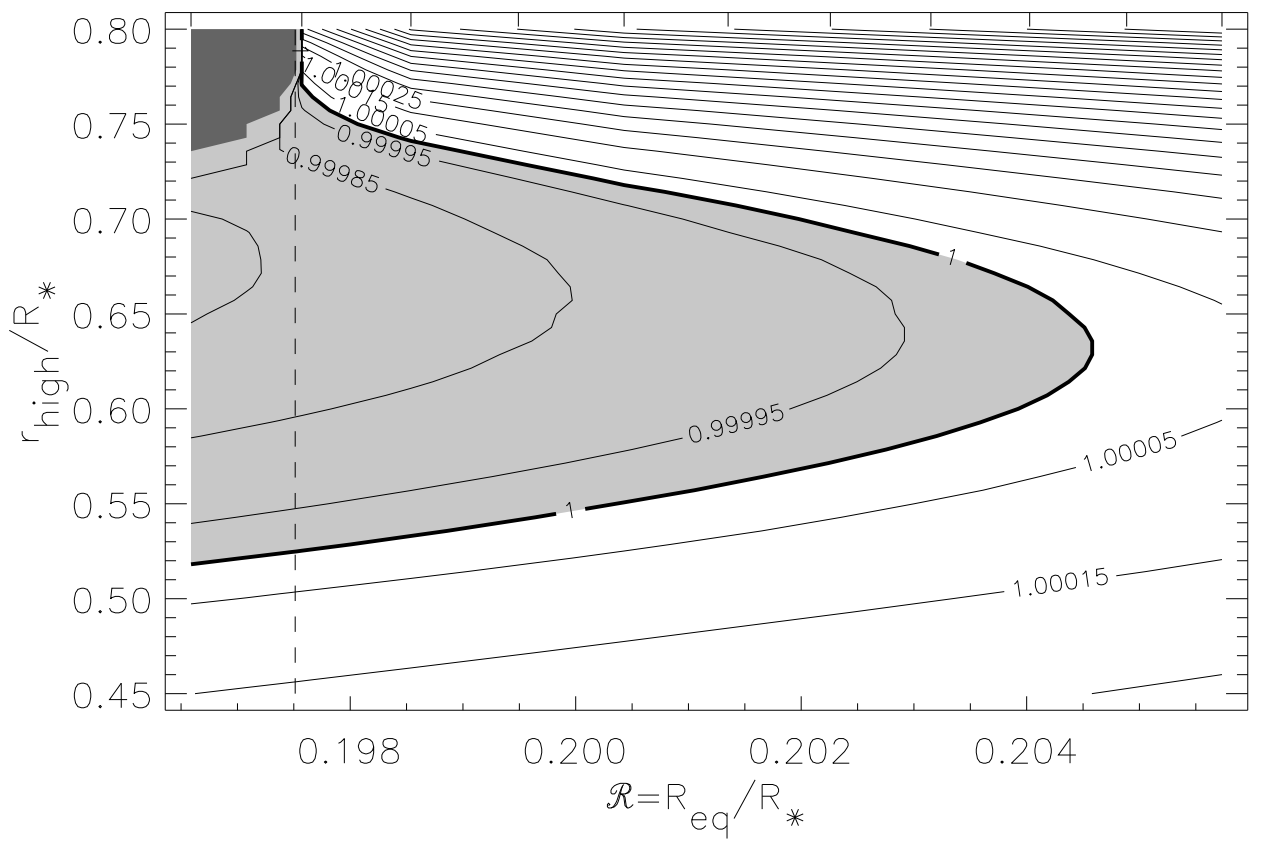

AbBILDUNG 5.4: Nicht-lineare Simulationen: Verhältnis $\left|f_{\text {out }} / f_{\text {in }}\right|$ am Gipfelelement $r_{\text {high }}$ aufsteigender Flussröhrenschleifen. Die Übergangsbreite (gestrichelte Linie) liegt bei $\lambda_{\text {eq }} \approx 29.5^{\circ}$. Der dunkelgraue Bereich in der oberen linken Ecke enthält keine Daten, da die gefangenen Flussröhren diese Höhe nicht mehr erreichen. 


\section{Kapitel 6}

\section{Diskussion}

\section{Relevanz für die Koronagrenze}

Der vollständige Einschluss magnetischer Flussröhren erfolgt in Sternmodellen des Massebereichs $M_{*}=1 \ldots 3 \mathrm{M}_{\odot}$ bei Oberflächentemperaturen von $T_{\text {eff }} \simeq 4750 \ldots 4900 \mathrm{~K}$. Sternmodelle vergleichbarer Entwicklungsstadien, d.h. mit ähnlichen Abmessungen des radiativen Kerns, die unter der Verwendung der OPAL Zustandsgleichung (Rogers et al. 1996) oder des Geneva-Toulouse-Codes (z.B. Charbonnel \& Talon 1999) berechnet wurden, sind im Vergleich zu den Standard-Entwicklungssequenzen (Tab. 4.1) um etwa $\left|\Delta T_{\text {eff }}\right| \lesssim 60 \mathrm{~K}$ verschoben (Abb. 4.1). Der auf Grundlage dieser Sternmodelle bestimmte Übergang von ausbrechenden Flussröhren zu gefangenen ist praktisch identisch mit dem der Standard-Simulationen, sodass der ermittelte Temperaturbereich recht stabil ist. Unter Verwendung von Umrechnungstabellen (Schmidt-Kaler 1982) ergibt sich daraus, dass der Einschluss magnetischer Flussröhren in Riesensternen (LC III) etwa im Spektralbereich G7 bis K0 einsetzt. Dieses Gebiet des HR Diagramms liegt sehr dicht links der Region, in der üblicherweise die Koronagrenze angesiedelt wird, dem Spektralbereich K1... K3. Dabei ist zu beachten, dass in der vorliegenden Behandlung nur Sterne untersucht wurden, die diese HR Region zum ersten Mal durchqueren und dann den RGB hinaufwandern. An der Spitze des RGB beginnen Sterne mit $M_{*} \lesssim 3 \mathrm{M}_{\odot}$ ihre Helium(Kern)Brennphase mit dem so genannten 'Helium Flash', wobei sie nochmal zurück zum Ansatz des RGB springen. Dadurch sammeln sich in diesem Bereich der so genannten 'Klumpenriesen' ( $K$ clump stars) verschiedene Sternpopulationen mit einem sehr heterogenen inneren Aufbau. Die entwickelten Post-Helium-Flash-Sterne wurden hier jedoch nicht berücksichtigt.

Ausbrechende Flussröhren führen zu bipolaren Fleckengruppen in der Photosphäre und zur Bildung großskaliger Magnetfeldbögen in der Sternatmosphäre, die den Einschluss von heißem $\left(T>10^{5} \ldots 10^{6} \mathrm{~K}\right)$ Plasma in der Übergangsschicht und Korona ermöglichen; insbesondere in ausgedehnten Riesensternen ist ein magnetischer Einschluss des Gases zur Aufrechterhaltung sehr hoher Temperaturen erforderlich. Da die Grenzlinien für das Auftreten einer Übergangsschicht (Linsky \& Haisch 1979) bzw. Korona (Ayres et al. 1981) im HR Diagramm mit dem Gebiet zusammenfallen, in dem ein signifikanter Massenverlust in Form langsamer, kühler Sternwinde einsetzt (Reimers 1977), wurde — neben verschiedenen anderen Szenarien — vermutet, dass der Rückgang koronaler Röntgenemission durch eine Änderung der atmosphärischen Magnetfeldstruktur verursacht wird. Rosner et al. (1991, 1995) und Kashyap et al. (1994) nehmen einen 

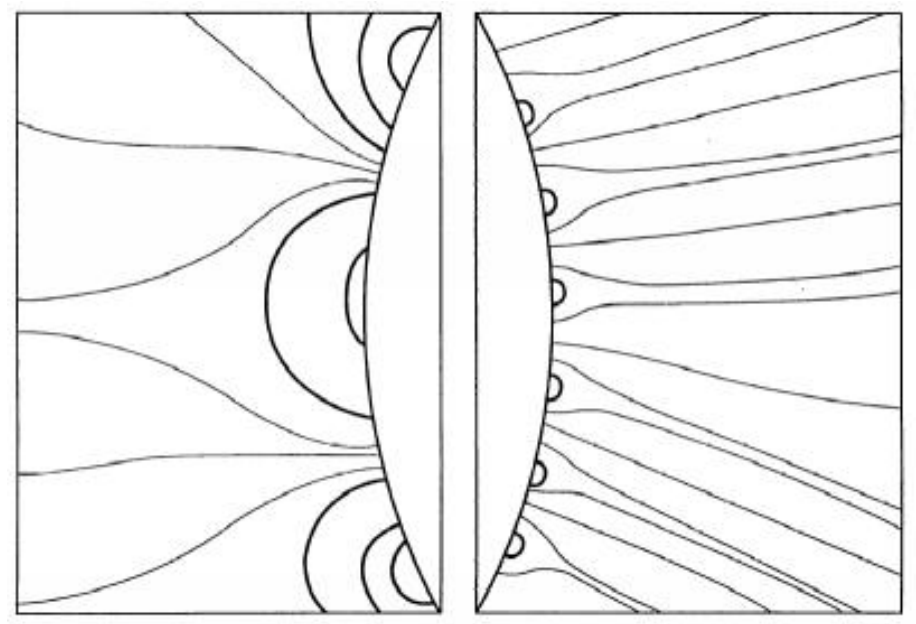

ABBILDUNG 6.1: Topologie des atmosphärischen Magnetfelds in kühlen Riesen zu beiden Seiten der CDL (nach Rosner et al. 1995). Auf der heißen Seite der CDL (links) dominieren großskalige, geschlossene Bögen, während auf der kühlen Seite (rechts) kleinere Bögen in überwiegend 'offenen' Magnetfeldstrukturen eingebettet sind, entlang derer ein massiver Sternenwind abströmen kann.

grundlegenden Übergang der Magnetfeld-Topologie an (vgl. Abb. 6.1): Von einer Magnetfeldstruktur, die durch eine Vielzahl großskaliger Koronabögen dominiert wird (auf der linken Seite der CDL), zu einer, in der Gebiete mit 'offenen' Feldlinien überwiegen und nur wenige kleinskalige Bögen existieren (auf der kühlen Seite). Die ausgedehnten, von offenen Feldlinien dominierten Oberflächenareale ermöglichen dabei das Abströmen eines kühlen Sternwindes, der (möglicherweise) von Alfvénwellen angetrieben wird. Als Ursache für diesen Übergang vermuten sie eine Änderung des Dynamoprozesses, der von einem sonnenähnlichen Mechanismus, der großskalige, toroidale Magnetfelder erzeugt, in einen übergeht, der auf den kleinen Längenskalen des lokalen konvektiv-turbulenten Geschwindigkeitfelds arbeitet und in der Lage ist ein geringes Maß an Aktivität aufrecht zu halten. Der Einschlussmechanismus magnetischer Flussröhren in Riesensternen unterstützt dieses Szenario, da er eine physikalische Grundlage für den Übergang der Feldtopologie liefert, bei der keine Änderung des zu Grunde liegenden Dynamomechanismus erforderlich ist. Die Überreste gefangener Flussröhren, die durch turbulente Gasbewegungen innerhalb der Konvektionszone aufgelöst werden, können darüber hinaus im Rahmen der Magneto-Konvektion durch einen lokalen Dynamoprozess verstärkt werden und dadurch zu einem gewissen Mindestmaß an Aktivität beitragen.

Unter Berücksichtigung der vergleichsweise schnellen Sternentwicklung im Riesenstadium entspricht der Flussröhreneinschluss aufgrund des Grenzkriteriums (4.1) einer vergleichsweisen abrupten Änderung der Oberflächenaktivität — verbunden mit einem entsprechend starken Rückgang der Röntgenemission. Demnach würde sich die Aktivität eines individuellen Sterns beim Überschreiten der CDL sprunghaft ändern. Dabei ist der Einschlussmechanismus unabhängig von der Rotationsrate und sollte, solange ein Dynamomechanismus vorhanden ist, bei allen Riesensternen entsprechender Entwicklungsstadien auftreten. Nach dem, beispielsweise von Hünsch \& Schröder (1996) vertretenen, Konzept einer Koronagrenzlinie aufgrund benachbarter Sternpopulationen mit unterschiedlichen Rotationsvergangenheiten, ändert sich die magnetische Aktivität hingegen in einem Ensemble ähnlicher Sterne (mit vergleichbaren Massen) kontinuierlich während ihrer Entwicklung entlang der CDL, d.h. ohne sprunghafte Änderungen der Aktivität. Eine Entscheidung zwischen den verschiedenen Ansätzen zur Erklärung der CDL könnte womöglich durch die eingehendere Beobachtungen einer größeren Zahl von Rie- 
sensternen in Verbindung mit präzisen Nach-Hauptreihen-Sequenzen getroffen werden, falls dadurch festgestellt werden kann, ob die Entwicklungswege die Koronagrenze kreuzen oder parallel zu ihr verlaufen.

\section{Die Rolle des Flussröhrenmodells}

Die Vernachlässigung von Wechselwirkungen verschiedener Flussröhren untereinander ist in Hinblick auf die pol- bzw. äquatorwärtige Drift des in der Overshoot-Region verankerten Röhrensegments vermutlich unrealistisch, da in dieser Situation mit Kontakten zwischen verschiedenen Flussröhren zu rechnen ist. Diese Interaktionen erschweren das Absinken gefangener Flussröhren zum Äquator. Es ist daher unwahrscheinlich, dass sie in der gleichmäßigen und kontinuierlichen Art und Weise zum Äquator driften wie in Abschn. 4.3 dargestellt. Und da die Zeit bis zum Erreichen des neuen Gleichgewichts eher lange ist, erreichen sie diesen Endzustand womöglich nie. Es ist im Grunde aber auch irrelevant, ob eine gefangene Flussröhre das äquatoriale Gleichgewicht erreicht, von konvektiven Strömungen zerstört wird, oder sich mit anderen Flussröhren verbindet. Der wesentliche Punkt ist die Verhinderung des Ausbruchs großskaliger Magnetfeldstrukturen an der Sternoberfläche. Da das grundsätzliche Problem von Magnetfeldern in Konvektionszonen ihre dauerhafte Speicherung und nicht ihr Ausbruch ist, führt der Einschlussmechanismus vermutlich nicht zu einem völligen Verschwinden magnetischer Aktivität, sondern eher zu einem drastischen Rückgang der Röntgenemission im Regime kühler Riesensterne.

Die Simulationen beinhalten weder differentielle Rotation noch meridionale Strömungen. Obwohl davon auszugehen ist, dass derartige Strömungen auch in Riesensternen existieren, führen die für das Einsetzen der Parker-Instabilität benötigten hohen Feldstärken zu magnetischen Energiedichten, welche die kinetischen Energiedichten dieser Strömungen deutlich übertreffen. Sobald eine aufsteigende Flussröhrenschleife in die Konvektionszone übergegangen ist, wird ihre weitere Dynamik im Wesentlichen durch Auftriebs- und magnetische Kräfte dominiert, sodass die tatsächliche Bewegung der Umgebung ihre Entwicklung kaum noch beeinflusst. Gleichermaßen würde eine sonnenähnliche differentielle Rotation innerhalb der Overshoot-Region die Stabilitätseigenschaften kaum verändern (Ferriz-Mas \& Schüssler 1995). Allerdings kann aufgrund des Fehlens schlüssiger Beobachtungen und verlässlicher theoretischer Modelle die Möglichkeit einer außergewöhnlich starken differentiellen Rotation in Riesensternen auch nicht ausgeschlossen werden. Der Einschlussmechanismus scheint allerdings weitgehend unabhängig von der Situation in der Overshoot-Region zu sein, da er, wie oben gezeigt wurde, durch die am Gipfelpunkt angreifenden Kräfte dominiert wird, der weit oberhalb des unteren Rands der Konvektionszone liegt.

Alle untersuchten Flussröhren beginnen ihre Entwicklung aus der gleichen Ausgangskonfiguration: Eine parallel zum Äquator liegende, toroidale Flussröhre im mechanischen Gleichgewicht innerhalb der Overshoot-Region. Obwohl es sich bei dieser Gleichgewichtsbedingung um eine - in Hinblick auf einen potenziellen $\alpha \Omega$-Dynamo und die Notwendigkeit der Speichung von Magnetfeldern — naheliegende Annahme handelt, kann die Existenz alternativer Gleichgewichte nicht grundsätzlich ausgeschlossen werden. Obwohl die Parameterstudie gezeigt hat, dass der Einschlussmechanismus sehr robust ist, müssen weitere Aspekte noch untersucht werden: Ist ein Einschluss magnetischer 
Flussröhren auch in massereicheren Sternen zu finden (Hybrid Sterne), in Klumpenriesen oder entwickelten Komponenten schnell-rotierender RS CVn-Doppelsternsystemen? Junge Vor-Hauptreihensterne besitzen auch kleine radiative Kerne und zeigen magnetische Aktivität. Kann der Einschlussmechanismus auch bei ihnen auftreten? 


\section{Teil II}

\section{Doppelsterne}





\section{Kapitel 7}

\section{Einleitung und Motivation}

Physikalische Doppelsterne sind eine sehr häufige Erscheinung. Zwar schwanken die Angaben über ihre statistische Verteilung je nach Art und Vollständigkeit des betrachteten Ensembles, aber man geht davon aus, dass nur etwa ein Drittel aller Sterne (im bürgerlichen, 'klassischen' Sinne) tatsächlich Einzelsterne sind (z.B. Abt \& Levy 1976, Duquennoy \& Mayor 1991). Seit ihrer ersten Identifizierung um 1780 durch Herschel $^{1}$ spielen diese Objekte in der Astronomie eine wichtige Rolle, da sie die einzige Möglichkeit einer direkten Massenbestimmung von Sternen darstellen und sich dadurch hervorragend zum Test theoretischer Sternaufbau- und Entwicklungsmodelle eignen. Daraus ergibt sich zwangsläufig ihre besondere Bedeutung auch für darauf aufbauende Theorien wie beispielsweise über die Massen- und Drehimpulsänderung von Sternen, deren (differentieller) Rotation und Konvektion und die daraus entstehenden Dynamomechanismen und magnetischen Aktivitätsphänomene.

Doppelsterne bilden etliche Klassen in der Gruppe der veränderlichen Sterne, für deren Verständnis allerdings die Kenntnis ihrer Systemparameter erforderlich ist. Im Gegensatz zu visuellen und astrometrischen Doppelsternen, deren Bahnelemente durch wiederholte Ortsbestimmungen der getrennt sichtbaren Komponente(n) bestimmt werden können, verwendet man bei engen, kurzperiodischen Systemen, die nicht mehr aufgelöst werden können, photometrische und spektroskopische Verfahren. Bei der Gruppe der Bedeckungsveränderlichen (EB, eclipsing binaries) wird beispielsweise der Verlauf des photometrischen Lichtwechsels während des Vorbeigangs einer Komponenten vor der anderen registriert. Bei spektroskopischen Doppelsternen (SB, spectroscopic binaries) wird dagegen die Intensität und das Profil charakteristischer Spektrallinien beobachtet, die wegen der periodischen Geschwindigkeitsänderungen der rotierenden Komponenten (entlang der Sichtlinie) eine Dopplerverschiebung bzw. -verbreiterung zeigen. Insbesondere bei halbgetrennten oder Kontaktsystemen kommt es zum Massentransfer zwischen den Komponenten, zur Ausbildung von Akkretionsscheiben oder gemeinsamen Atmosphären, welche die Interpretation der Beobachtungen erschweren. Diese Effekte spielen bei den im Weiteren betrachteten getrennten Systemen keine Rolle, aber auch hier wird die Analyse der Licht- und Spektralkurven durch sekundäre Einflüsse erschwert. Dies sind, um nur einige zu nennen, die Rand- und Gravitationsverdunkelung, die Gezeitenverformung der Komponenten, Reflexionseffekte aufgrund der gegenseitigen Bestrahlung und

\footnotetext{
${ }^{1}$ Friedrich Wilhelm Herschel, deutscher Astronom, 1738 - 1822
} 
phasenabhängige Helligkeitsschwankungen aufgrund von Temperaturinhomogenitäten in verschiedenen Atmosphärenschichten.

Die eigentlichen Oberflächenstrukturen und -inhomogenitäten weit entfernter Sterne können natürlich nicht direkt beobachtet werden. Aber mit Modellannahmen für die Helligkeitsinhomogenitäten ist es möglich, aus der photometrischen Lichtkurve in einem Inversionsprozess deren grundsätzliche Charakteristika abzuleiten; bei dunklen Sternflecken beispielsweise ihre Anzahl, Größe, Lage und Temperatur (z.B. Eaton \& Hall 1979, Rodonò et al. 1986, Budding \& Zeilik 1987). Dazu werden auch ältere und z.T. mehrere Jahrzehnte zurückreichende Beobachtungen verwendet, um Aufschlüsse über Aktivitätszyklen zu erhalten (Rodonò et al. 2000). Weiter entwickelte Rekonstruktionsmethoden wie das Doppler Imaging (DI)-Verfahren verwenden zusätzlich zur Lichtkurve rotationsmodulierte, asymmetrische Linienprofile, beispielsweise von Fe I oder Ca I, die durch die Überlagerung der Beiträge verschiedener Oberflächenelemente mit unterschiedlichen Dopplerverschiebungen entstehen Die Inversionstechniken sind allerdings nicht eindeutig und benötigen daher Regularisierungsbedingungen, um eine Lösung für die Verteilung dunkler oder heller Flecken zu liefern (Vogt \& Penrod 1983, Vogt et al. 1987). Abweichungen der Ergebnisse bei Verwendung verschiedener Regularisierungsfunktionen werden jedoch mit besserem Signal-zu-Rausch-Verhältnis der Ausgangsdaten immer geringer. Durch die Verwendung magnetisch sensitiver Spektrallinien kann beim Zeeman-DI das Magnetfeld und dessen Orientierung bestimmt und damit die der Lichtmodulation zugrundeliegende, magnetische Ursache nachgewiesen werden (Semel 1989, Donati et al. 1992).

\section{Magnetische Aktivität in Doppelsternen}

Schnelle Rotation und eine ausgedehnte Konvektionszone sind die Voraussetzungen für einen effektiven Dynamomechanismus. Beides ist in engen Doppelsternsystemen vorhanden, wenn mindestens eine kühle Komponente vom Spektraltyp G - M vorhanden ist. Während vergleichbare Einzelsterne in ihrem Hauptreihenstadium aufgrund der magnetischen Abbremsung eine deutliche Verringerung ihrer Rotation erfahren, ist in engen Systemen mit Perioden von $T \lesssim 12 \mathrm{~d}$ aufgrund der Gezeitenwechselwirkung die Sternrotation i.Allg. mit der Dauer des Bahnumlaufs synchronisiert. Dadurch ist in einer Komponente, die entweder auf der Hauptreihe schon eine äußere Konvektionszone besitzt oder während ihrer Nach-Hauptreihen-Entwicklung eine solche ausbildet, eine effiziente Magnetfeldverstärkung möglich, die zu wesentlich ausgeprägteren Aktivitätserscheinungen führt als sie bei der Sonne beobachtet werden. Die vielfältigen Kombinationsmöglichkeiten der Konfigurationen und Entwicklungsstadien beider Komponenten führen zu verschiedenen Klassen magnetisch aktiver Doppelsterne, von denen hier nur einige aufgeführt werden:

- W UMa-Systeme sind kurz-periodische $(T \lesssim 1 \mathrm{~d}$ ) bedeckungsveränderliche Sterne nahe der Hauptreihe vom Spektraltyp F - M in einem Kontaktsystem, meist mit gemeinsamen Atmosphären. Die Struktur der stark elliptisch verformten Komponenten führt zu komplizierten Lichtkurven, für deren Erklärung auch die Existenz dunkler Sternflecken mit herangezogen wird (Eaton et al. 1980). 
- AM Her-Systeme oder Polars gehören zur Gruppe der Kataklysmischen Veränderlichen $(C V)$. Sie haben Rotationsperioden von nur wenigen Stunden und zeigen plötzliche Ausbrüche von bis zu 5 mag, die durch die Akkretion von einem kühlen Hauptreihenstern abströmender Materie auf einen magnetischen Weißen Zwerg erzeugt wird, dessen starkes Magnetfeld mit Feldstärken der Größenordnung $10^{7} \ldots 10^{8} \mathrm{G}$ die Ausbildung einer Akkretionsscheibe verhindert, indem es den Materiestrom zu einem Fleck in der Nähe des Magnetpols dirigiert (Gaensicke et al. 1995, Fischer \& Beuermann 2001). Neben starker Emission im weichen Röntgenbereich zeigen diese Systeme eine veränderliche lineare und zirkulare Polarisation im Optischen und UV.

- UV Cet- oder Flaresterne sind kühle Zwergsterne vom Spektraltyp dMe, meist mit Wasserstoffemissionslinien, deren optische Helligkeit aufgrund unregelmäßiger Flares innerhalb von Sekunden bis Minuten um bis zu 7 mag ansteigen kann. Sie zeigen weitere Anzeichen sonnenähnlicher Aktivität, wie dunkle Flecken und variable chromosphärische und koronale Emission in Form von UV-, Röntgen- und Radiobursts (Gershberg et al. 1999, und darin enthaltene Zitate). Sie treten häufig auch als Einzelsterne auf.

- $R S C V n$ - und BY Dra-Sterne sind enge, aber getrennte Doppelsterne, deren photometrische Lichtkurven außerhalb der Bedeckungen periodische Schwankungen aufweisen (photometric distortion wave), die mit ausgedehnten Inhomogenitäten der Oberflächenhelligkeit erklärt werden. Sie gehören zur Gruppe chromosphärisch aktiver Sterne (Strassmeier et al. 1993, und darin enthaltene Zitate) und zeigen darüber hinaus noch viele weitere Merkmale magnetischer Aktivität. BY Dra-Sterne bilden beispielsweise eine Untergruppe der Flaresterne.

Im Gegensatz zu Kontaktsystemen oder Kataklysmischen Veränderlichen haben getrennte Systeme den Vorteil, dass Beobachtungen nicht durch stark deformierte Komponenten, gemeinsame Atmosphären, einen überfließenden Materiestrom oder eine Akkretionsscheibe beeinflusst werden. Aus diesem Grund spielen RS CVn- und BY Dra-Systeme eine besondere Rolle, weil sie eine vergleichsweise einfache Untersuchung intrinsischer Sternaktivität erlauben, deren diversen Signaturen wesentlich stärker ausgeprägt sind als bei der Sonne.

\section{RS CVn- und BY Dra-Sterne...}

In RS CVn-Systemen befindet sich mindestens eine (aktive) Komponente in einem fortgeschrittenen Entwicklungsstadium, etwa ein kühler Riese (LC III) oder Unterriese (LC IV) vom Spektraltyp G - K. Periodische Variationen ihrer Lichtkurve außerhalb der Bedeckungen werden in Analogie zur Sonne auf dunkle Sternflecken und helle Fackelgebiete zurückgeführt. Chromosphärische Aktivität macht sich in Form von Plages, Flares, verstärkter $\mathrm{H} \alpha$-Emission und in der rotationsperiodischen Modulation von Ca II H \& $\mathrm{K}$ und Mg II h \& k-Linienkernen bemerkbar. Analysen von $\mathrm{H} \alpha$-Linienprofilen zeigen Signaturen vergleichsweise kühler Materie, die in der deutlich heißeren Sternatmosphäre eingebettet ist und solaren Protuberanzen ähnelt (z.B. Collier Cameron 1996, Jardine et al. 
2001). Dies lässt, in Analogie zur Sonne, auf die Existenz ausgedehnter, großskaliger Magnetfelder in geschlossenen Bögenstrukturen schließen, die einzelne Aktivitätsregionen miteinander verbinden und die Aufheizung und den Einschluss koronalen Plasmas ermöglichen. Die Strahlungsflüsse von kurzperiodischen RS CVn-Systemen im EUV und Röntgenbereich liegen mehrere Größenordnungen über denen vergleichbarer, aber langsamer rotierender Einzelsterne; sie gehören zu den stärksten Röntgenquellen im Regime später Spektraltypen (Dempsey et al. 1997, Osten \& Brown 1999). Die irreguläre Variabilität wird im Wesentlichen durch Flare-Ereignisse verursacht, die sich in allen Spektralbereichen und von der Chromosphäre bis zur Korona bemerkbar machen. Die dabei freigesetzte magnetische Energie ist für die starke Beschleunigung von Teilchen und die daraus folgenden nicht-thermischen und teilweise polarisierten Radiobursts verantwortlich (z.B. Osten et al. 2000).

Hall (1976) hat bei seiner Klassifizierung der RS CVn-Systeme verschiedene Untergruppen unterschieden, so z.B. reguläre Systeme mit Rotationsperioden $T=1 \ldots 14 \mathrm{~d}$, langperiodische $(T>14 \mathrm{~d})$ und kurzperiodische $(T \lesssim 1 \mathrm{~d})$. Kurzperiodische RS CVnSysteme scheinen im Gegensatz zu den anderen aus Hauptreihensternen zu bestehen, die ihr Rochevolumen beinahe ausfüllen und manchmal als BY Dra-Systeme eingestuft werden (Fekel et al. 1986, Budding \& Zeilik 1987). BY Dra-Veränderliche wurden ursprünglich als schnell rotierende dKe - dMe Hauptreihensterne (LC V) mit Ca II H \& K Emissionslinien klassifiziert, die aufgrund von Sternflecken periodisch variierende Lichtkurven haben. Inzwischen gilt es als sicher, dass der Großteil (vielleicht sogar alle) dieser Objekte Doppelsternsysteme mit Hauptreihensternen der Spektraltypen F - M sind (Bopp \& Fekel 1977, Fekel et al. 1986). Eine umfassende Zusammenstellung chromosphärisch aktiver Doppelsterne findet man bei Strassmeier et al. (1993).

\section{...und ihre Fleckenverteilungen}

Die aus photometrischen und spektroskopischen Daten rekonstruierten Fleckenverteilungen (siehe z.B. Abb. 7.1) auf schnell rotierenden Doppelsternen unterscheiden sich sowohl quantitativ als auch qualitativ deutlich von dem uns vertrauten Bild der Sonnenphotosphäre. Während bei dieser die größten Fleckengruppen weniger als $0.1 \%$ der gesamten Oberfläche einnehmen, können bei aktiven Doppelsternen $20 \%$ und mehr von Sternflecken bedeckt sein (z.B. O'Neal et al. 1998, Lanza et al. 2001). Ein weiterer Unterschied zeigt sich in der Fleckenanordnung. In der Sonne treten Fleckengruppen nur in einem äquatornahen Bereich zwischen etwa $\pm 35^{\circ}$ Breite auf. In schnell rotierenden Sternen liegen Ausbruchsorte dagegen auch bei deutlich höheren und gar höchsten Breiten. Ein typisches Charakteristikum schneller Rotatoren ist das Vorhandensein ausgedehnter polnaher bzw. polarer Flecken (polar caps), die der Wirkung der Corioliskraft auf aufsteigende magnetische Flussröhren zugeschrieben werden (Schüssler \& Solanki 1992). Da sich polare Flecken bei der Rotationsmodulation der Lichtkurve und Linienprofile kaum bemerkbar machen und Beobachtungen mit einem sehr guten Signal-zu-Rausch-Verhältnis voraussetzen, ist ihr Nachweis aber schwierig und teilweise kontrovers. Da in das Inversionsverfahren die Neigung der Bahnebene eingeht und die Bestimmung der Breitenverteilung von Sternflecken empfindlich von dieser Größe abhängt, ist eine genaue Bestimmung der Bahn- und Systemparameter unerlässlich. Die Ermittlung der Längenverteilung ist dage- 


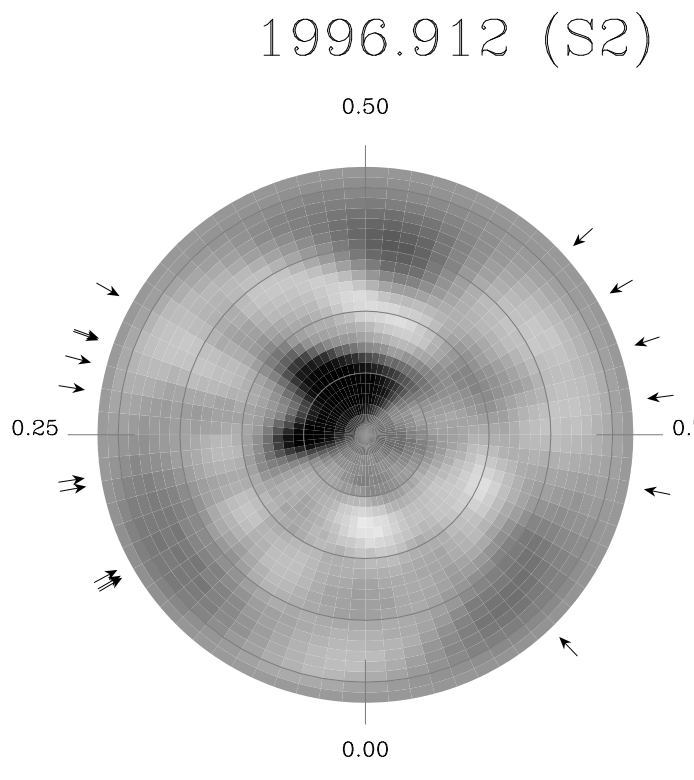

AbBildung 7.1: Aus CaI-Beobachtungen bei $6439 \AA$ \& rekonstruierte Oberflächenstrukturen der aktiven K1 IV Komponente des Systems V711 (HR 1099) mit einer Umlaufdauer von $T=2.8 \mathrm{~d}$. Polare Projektion des Breitenintervalls $\lambda=+90 \ldots-40^{\circ}$. Breitenkreise markieren Schritte von $\Delta \lambda=$ $30^{\circ}$ und Pfeile am Rand die Phasen der spektroskopischen Beobachtungen. Auffallend ist besonders der dunkle asymmetrische Fleck in Polnähe mit deutlich tieferen Temperaturen als der Rest der Oberfläche (Nach Strassmeier \& Bartus 2000).

gen etwas einfacher, da zumindest bei Bedeckungsveränderlichen durch die Minima der Lichtkurve eine exakte Bestimmung und Zuordnung der Rotationsphase möglich ist.

Deutliche Asymmetrien in longitudinalen Verteilungsfunktionen weisen darauf hin, dass Sternflecke nicht gleichmäßig über alle Längen verteilt sind, sondern sich an manchen häufen bzw. vermehrt auftreten (z.B. Henry et al. 1995, Jetsu 1996, und darin enthaltene Zitate). Solch beständige Vorzugslängen werden manchmal auch als 'Aktivitätsgürtel' (ALBs, active longitude belts) bezeichnet. Neben Fällen, in denen keine, mehrere oder unregelmäßig verteilte Vorzugslängen zu finden sind, treten häufig Längenverteilungen auf, bei denen zwei ALBs um $\sim 180^{\circ}$ voneinander getrennt in gegenüberliegenden Quadranten des Sterns liegen. Die Orientierung dieser Aktivitätszentren relativ zum Begleitstern ist unterschiedlich: In kurzperiodischen Systemen mit Umlaufsdauern $T \lesssim 1 \mathrm{~d}$ scheinen ALBs bevorzugt in der Umgebung der Quadraturpunkte zu liegen, d.h. in den Richtungen senkrecht zur Verbindungslinie Stern - Begleiter (Zeilik et al. 1989, 1990b,a, 1994, Zeilik 1992, Olah et al. 1994, Heckert et al. 1998). In längerperiodischen Systemen ist die Situation hingegen uneinheitlich. Neben beständigen Vorzugslängen um den substellaren Punkt, d.h. der Richtung zum Begleitstern, und/oder dessen Antipoden zeigen manche Sterne ALBs auch bei anderen Längen (Lanza et al. 1998, 2001, 2002). Häufig zeigt die photometrische Lichtkurve eine zeitliche Phasenverschiebung ihrer fleckenbedingten Modulation, welche eine Migration der Aktivitätsherde anzeigt (Strassmeier 1994, Rodonò et al. 1995, Berdyugina \& Tuominen 1998, Berdyugina et al. 1999, Rodonò et al. 2000). Sie wird auf eine differentielle Rotation des Sterns zurückgeführt, die bei schnell rotierenden Komponenten enger Doppelsternsysteme aber deutlich geringer als im Fall der Sonne ist (Hall 1991).

Ausgehend von einer Untersuchung von ALBs in Systemen mit unterschiedlichen Rotationsperioden, ziehen z.B. Heckert \& Ordway (1995) die Möglichkeit in Betracht, dass die Lage von Vorzugslängen von der Gravitationswirkung des Begleitsterns beeinflusst wird; denkbar ist etwa der Übergang von festen zu migrierenden Vorzugslängen beim Übergang von kurzen zu längeren Rotationsperioden. Untersuchungen magneti- 
scher Flussröhren beschränkten sich bisher auf Einzelsterne, in denen Axialsymmetrie bezüglich der Rotationsachse vorliegt. In Doppelsternsystemen wird jedoch durch die Gezeitenwirkung eine weitere Richtung ausgezeichnet und die Axialsymmetrie gebrochen. Die Dynamik magnetischer Flussröhren wird dabei nicht nur durch die Gezeitenkraft selbst, sondern auch durch die breiten- und längenabhängige Variation der Sternschichtung beeinflusst. Um die Wirkung dieser Effekte auf aufsteigende Flussröhren zu untersuchen, wird nun das solare Paradigma magnetischer Aktivität auf enge Doppelsterne übertragen, und das Flussröhren-Modell um die Gezeitenwirkung eines Begleitsterns erweitert. Die Untersuchung umfasst dabei das Gleichgewicht, die Stabilität und die nichtlineare Entwicklung magnetischer Flussröhren durch die Konvektionszone bis zu ihrem Ausbruch an der Oberfläche. 


\section{Kapitel 8}

\section{Doppelsternmodell}

\subsection{Näherung für die Gezeitenwirkung}

In engen Doppelsternsystemen bewirken die Gezeitenkräfte eine Zirkularisation der Bahnbewegung und Synchronisation der Sternrotation mit dem Umlauf der Komponenten $^{1}$. Theoretische Vorstellungen über den Ablauf dieser Vorgänge beruhen auf der Dissipation turbulenter Gasbewegungen in der Konvektionszone durch viskose Reibung (Zahn 1977, Hut 1981) bzw. auf durch Gezeiteneffekte induzierte meridionale Strömungen, welche zu einer Umverteilung des Drehimpulses innerhalb des Sterns führen (Tassoul \& Tassoul 1992, Tassoul 1995). Beide Mechanismen laufen auf Zeitskalen ab, die im Vergleich zur Entwicklungszeitskala der Sterne sehr kurz sind. Statistische Untersuchungen belegen, dass die Bahnen enger Systeme mit Rotationsperioden von $T \lesssim 10$ d typischerweise keine Exzentrizität aufweisen (Duquennoy \& Mayor 1991) und die Rotation in sehr guter Näherung synchronisiert ist (Strassmeier et al. 1993). Aus diesem Grund wird im Weiteren von einem Doppelstern ausgegangen, in dem sich die Komponenten in gebundener Rotation auf Kreisbahnen gleichförmig um den gemeinsamen Massenschwerpunkt bewegen; im mitrotierenden Bezugssystem ist diese Systemkonfiguration zeitunabhängig.

\subsubsection{Effektives Potenzial und Gezeitenbeschleunigung}

Das hier verwendete Modell eines Doppelsternsystems besteht aus einem Hauptstern der Masse $M_{*}$ am Ort $\mathbf{r}_{*}$ und einem Begleitstern der Masse $M_{\text {co }}$ am Ort $\mathbf{r}_{\mathrm{co}}=\mathbf{r}_{*}+\mathbf{a}$ (vgl. Abb. 8.1). Die dimensionslose Größe $q=M_{\mathrm{co}} / M_{*}$ bezeichnet das Massenverhältnis der beiden Komponenten und $a=|\mathbf{a}|$ den Abstand zwischen den jeweiligen Sternmittelpunkten. Der Theorie des Zwei-Körper-Problems folgend gelten für die Sternorte im Schwerpunktsystem die Beziehungen

$$
\mathbf{r}_{*}=-\frac{M_{\mathrm{co}}}{M_{*}+M_{\mathrm{co}}} \mathbf{a}=-\frac{q}{1+q} \mathbf{a} \quad \text { und } \quad \mathbf{r}_{\mathrm{co}}=\frac{M_{*}}{M_{*}+M_{\mathrm{co}}} \mathbf{a}=\frac{1}{1+q} \mathbf{a} .
$$

\footnotetext{
${ }^{1}$ Ein System ist im stabilen Gleichgewicht, wenn sich die beiden Komponenten in Korotation auf ebenen Kreisbahnen bewegen und die Rotationsachsen parallel zueinander sind (Hut 1980).
} 


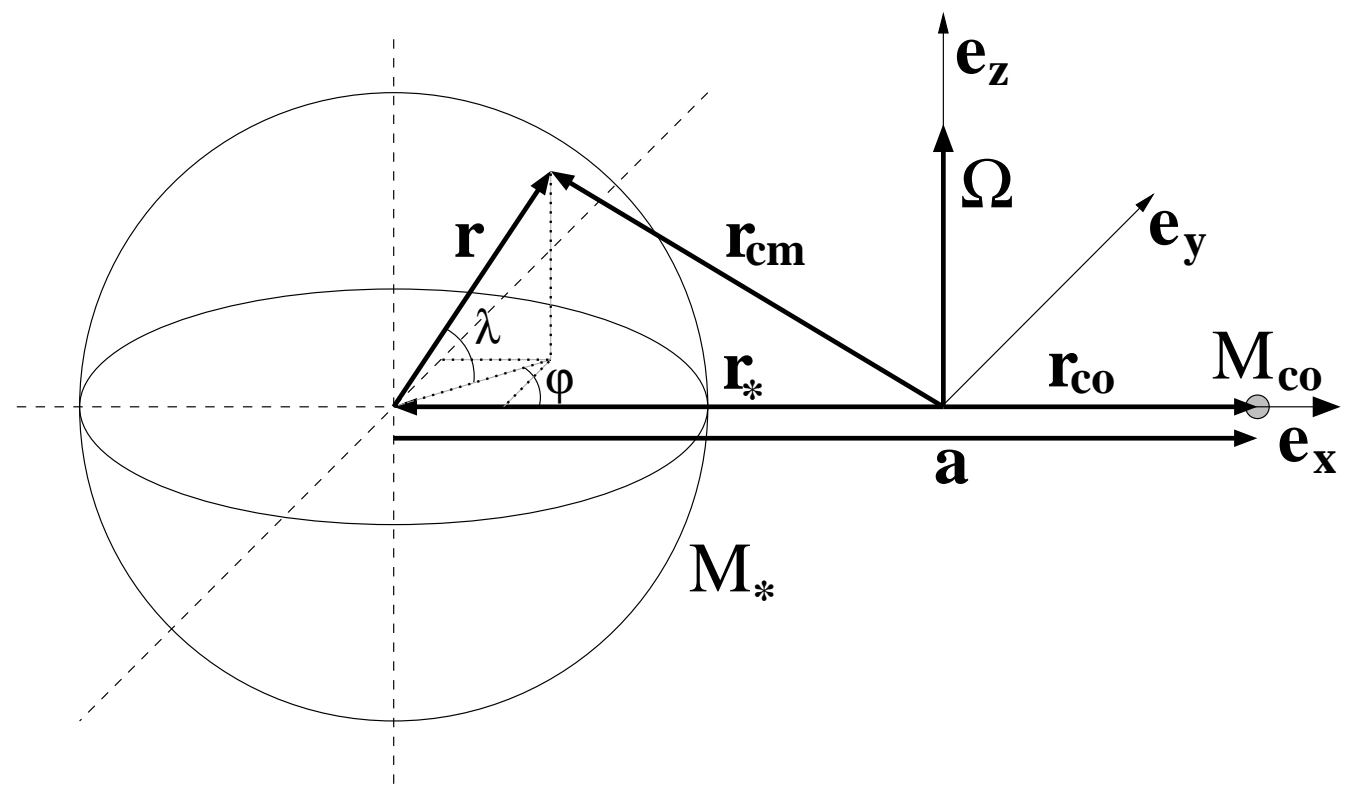

ABBILDUNG 8.1: Modell eines Doppelsternsystems. Der Ursprung des mitrotierenden Bezugssystems ist der Massenschwerpunkt. Aufgrund der Beziehungen (8.1) ist es möglich, in ein (Kugelkoordinaten-)System überzugehen, dessen Ursprung im Zentrum des Hauptsterns $M_{*}$ liegt. Dabei ist $r$ der Abstand vom Sternmittelpunkt, $\phi$ der Azimutwinkel und $\lambda$ der Winkelabstand vom Äquator.

Für eine gegebene Umlaufsperiode $T$ folgt aus dem 3. Keplerschen Gesetz der Abstand

$$
\left(\frac{a}{\mathrm{R}_{\odot}}\right) \simeq 4.207\left(\frac{M_{*}}{\mathrm{M}_{\odot}}\right)^{1 / 3}(1+q)^{1 / 3}\left(\frac{T}{\mathrm{~d}}\right)^{2 / 3} .
$$

Abbildung 8.2 zeigt den Verlauf des Abstands als Funktion der Umlaufsperiode $T$; die gestrichelte Linie gilt für das in in Abschn. 8.2 beschriebene Referenzsystem mit $M_{*}=$ $1 \mathrm{M}_{\odot}$ und $q=1$. Die Rotationsrate des Hauptsterns ist identisch mit der Umlauffrequenz und seine Rotationsachse parallel zu der des Systems:

$$
\Omega_{*}=\Omega_{\mathrm{sys}} \equiv \Omega \mathbf{e}_{\Omega}=\frac{2 \pi}{T} \mathbf{e}_{z}
$$

Das effektive Potenzial $\Psi_{\text {eff }}$ eines mit der Winkelgeschwindigkeit $\Omega$ rotierenden Doppelsterns wird im mitrotierenden Bezugssystem durch die Poisson-Gleichung

$$
\Delta \Psi_{\mathrm{eff}}=-4 \pi G \rho+2 \Omega^{2}
$$

bestimmt. Eine umfassende Darstellung der theoretischen Lösungsansätze für dieses Problem findet man bei Kopal (1959) und in deutlich kürzer Form bei Claret \& Gimenez (2001). Für die hier angestrebte Betrachtung ist die exakte Lösung von Gl. (8.4) allerdings nicht notwendig. Als Näherung wird stattdessen die Superposition eines einfachen Massenpunkt- und eines Einzelsternmodells verwendet. Das effektive Potenzial setzt sich 


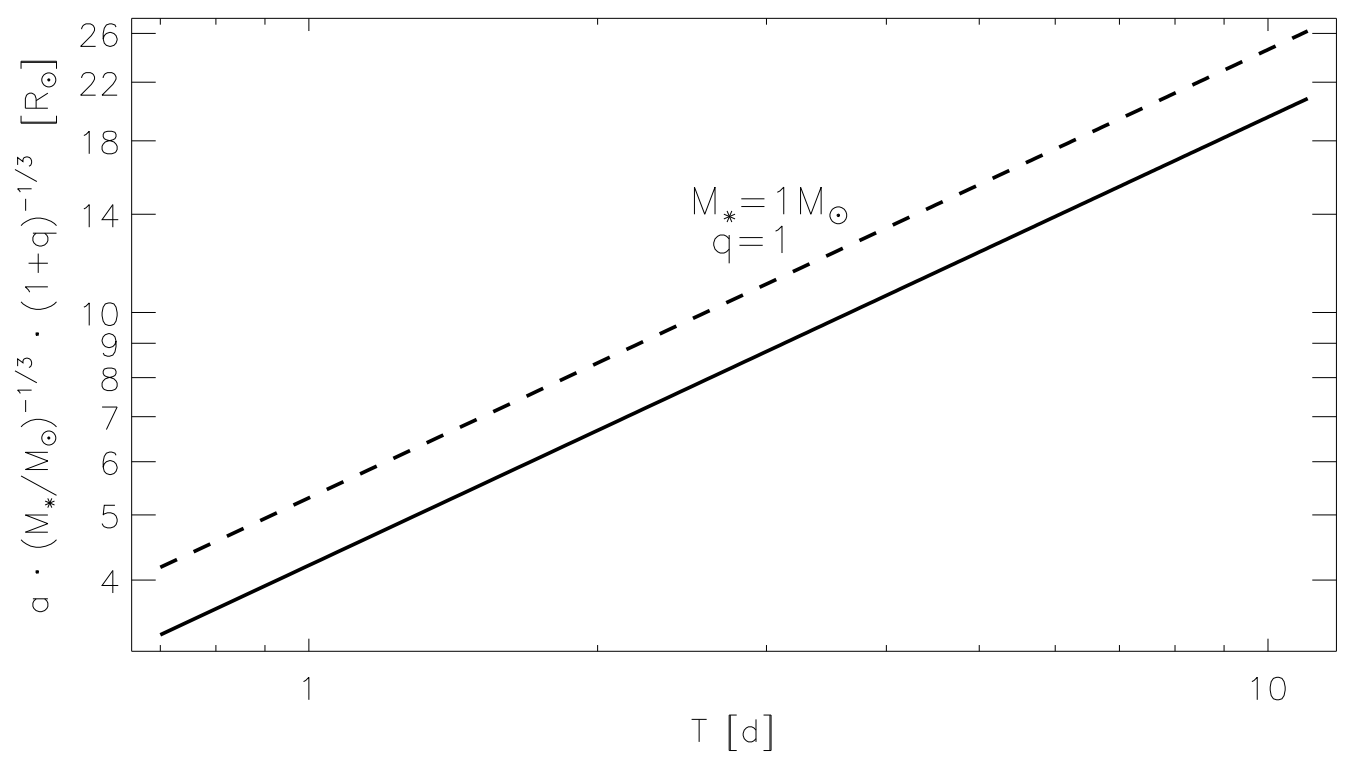

AbBiLdung 8.2: Sternabstand $a$ in einem zirkularisierten Doppelsternsystem mit der Umlaufsperiode $T$. In der doppelt-logarithmischen Darstellung sind Kurven für verschiedene Werte von $q$ und $M_{*}$ zu der durchgezogenen Linie parallel verschoben. Als Beispiel gibt die gestrichelte Linie den Verlauf für ein System mit $M_{*}=1$ und $q=1$ wieder.

aus den Gravitationspotenzialen des Haupt- und Begleitsterns $\Psi_{*}$ bzw. $\Psi_{\text {co }}$, und dem Rotationspotenzial $\Psi_{\text {rot }}$ zusammen:

$$
\begin{aligned}
\Psi_{*} & =\int_{0}^{r} G \frac{M(r)}{r^{2}} \mathrm{~d} r \\
\Psi_{\mathrm{co}} & =-G \frac{M_{*} q}{|\mathbf{r}-\mathbf{a}|}=-G \frac{M_{*} q}{a} \sum_{j=0}^{\infty}\left(\frac{r}{a}\right)^{j} P_{j}(\cos \gamma) \\
\Psi_{\text {rot }} & =-\frac{1}{2}\left|\boldsymbol{\Omega} \times \mathbf{r}_{\mathrm{cm}}\right|^{2}
\end{aligned}
$$

Dabei ist $M(r)$ die innerhalb einer Kugel mit dem Radius $r$ enthaltene Masse des Hauptsterns mit $M\left(R_{*}\right)=M_{*}$. Der explizite Verlauf dieser Schichtung wird einem berechneten Einzelsternmodell entnommen, der Begleitstern hingegen als Punktmasse angesehen. Die Legendre-Polynome $P_{j}$ in Gl. (8.6) sind Funktionen des Winkels $\gamma$, der durch die Beziehung

$$
\cos \gamma=\left(\mathbf{e}_{r} \cdot \mathbf{e}_{a}\right)=\cos \phi \cos \lambda
$$

mit Hilfe des Azimutwinkels $\phi$ (mit Nullpunkt in der Richtung zum Begleitstern) und der Breite $\lambda$ (Winkelabstand von der Äquatorebene) definiert wird. Dabei sind $\mathbf{e}_{r}$ und $\mathbf{e}_{a}$ normierte Richtungsvektoren vom Sternmittelpunkt zum Aufpunkt $(r, \phi, \lambda)$ bzw. zum Begleitstern. Im Rotationspotenzial (8.7) tritt der Ortsvektor $\mathbf{r}_{\mathrm{cm}}$ bezüglich des Massenschwerpunkts des Systems auf. Aufgrund der Beziehung $\mathbf{r}_{\mathrm{cm}}=\mathbf{r}_{*}+\mathbf{r}$ kann es mit Hilfe der Gl. (8.1) und dem 3. Keplerschen Gesetz in folgende Form gebracht werden:

$$
\Psi_{\text {rot }}=-\frac{1}{2}|\Omega \times \mathbf{r}|^{2}+G \frac{M_{*} q}{a}\left(\frac{r}{a}\right) P_{1}(\cos \gamma)+\text { const. }
$$



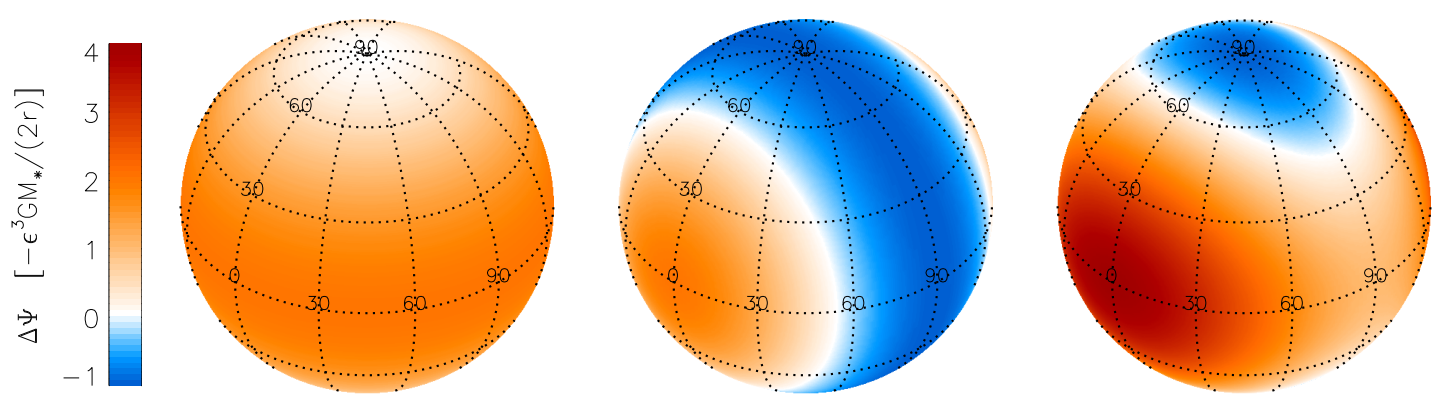

AbBILDUNG 8.3: Struktur der Potenzialänderung aufgrund der Rotation $\left(\Delta \Psi=\Psi_{\text {rot }}\right.$, links), des Begleitsterns $\left(\Delta \Psi=\Psi_{\text {co }}\right.$ mit $q=1$, Mitte $)$ und der Summe beider Teilpotenziale ( $\Delta \Psi=\Psi_{\text {tide }}$, rechts).

Der zweite Term dieser Gleichung ist bis auf das Vorzeichen identisch mit dem $j=$ 1-Term des Gravitationspotenzials (8.6) des Begleitsterns. Bei der Summenbildung der Teilpotenziale (8.5) - (8.7) heben sich diese beiden Terme weg und man erhält für das effektive Potenzial die Form

$$
\begin{aligned}
\Psi_{\mathrm{eff}} & =\Psi_{*}(r)+\Psi_{\text {tide }}(r, \phi, \lambda) \\
& =\Psi_{*}-\epsilon^{3} \frac{G M_{*}}{2 r}\left[\left(1+\frac{5}{2} q\right) \cos ^{2} \lambda-q+\frac{3}{2} q \cos ^{2} \lambda \cos 2 \phi\right]+\mathcal{O}\left(\epsilon^{4}\right) .
\end{aligned}
$$

Diese Gleichung entspricht einer Taylorentwicklung des effektiven Potenzials nach dem Abstandsverhältnis

$$
\epsilon=\frac{r}{a}
$$

bei der nur der erste nicht-verschwindende Term in $\epsilon$ berücksichtigt wird. Terme der Ordnung $\epsilon^{4}$ und höher werden hingegen vernachlässigt.

Gegenstand der Untersuchung sind Doppelsternsysteme mit $M_{*} \sim 1 \mathrm{M}_{\odot}, q \sim 1, T \sim$ $1 \ldots 10 \mathrm{~d}$ und, dem Keplergesetz (8.2) zufolge, Abständen $a \sim 5 \ldots 25 \mathrm{R}_{\odot}$. Da sich alle weiteren Untersuchungen auf Punkte innerhalb des Hauptsterns (mit $r \lesssim \mathrm{R}_{\odot}$ ) beziehen, gilt

$$
\epsilon^{3}=\left(\frac{r}{a}\right)^{3} \sim 10^{-2} \ldots 10^{-5}
$$

Dementsprechend ist die vom Begleitstern und der Rotation bewirkte Potenzialänderung $\Psi_{\text {tide }}$ nur eine kleine Störung des Einzelsternpotenzials $\Psi_{*}$. In Abb. 8.3 ist die Struktur der Teilpotenziale und ihrer Summe wiedergegeben.

Die aus den Teilpotenzialen (8.5) - (8.7) resultierende effektive Gravitationsbeschleunigung $\mathbf{g}_{\text {eff }}$ setzt sich zusammen aus der Eigengravitation $\mathbf{g}_{*}(r)$ des Hauptsterns und der Gezeitenkraft $\mathbf{g}_{\text {tide }}(r, \phi, \lambda)$. Ebenso wie bei der Störung des Potenzials (8.10) wird auch hier nur der führende $\epsilon^{3}$-Term berücksichtigt:

$$
\mathbf{g}_{\text {eff }}(r, \phi, \lambda)=-g_{*}\left[\mathbf{e}_{r}-\sigma \epsilon^{3}\left(\mathbf{e}_{r}+3 q\left(\mathbf{e}_{r} \cdot \mathbf{e}_{a}\right) \mathbf{e}_{a}-(1+q)\left(\mathbf{e}_{r} \cdot \mathbf{e}_{\Omega}\right) \mathbf{e}_{\Omega}\right)\right]
$$

Aufgrund der Gezeitenkraft hat die effektive Gravitation nicht nur eine Komponente in sphärisch-radialer Richtung $\mathbf{e}_{r}$, sondern auch Beiträge entlang der Breiten- und Längenrichtungen $\mathbf{e}_{\lambda}$ bzw. $\mathbf{e}_{\phi}$. 


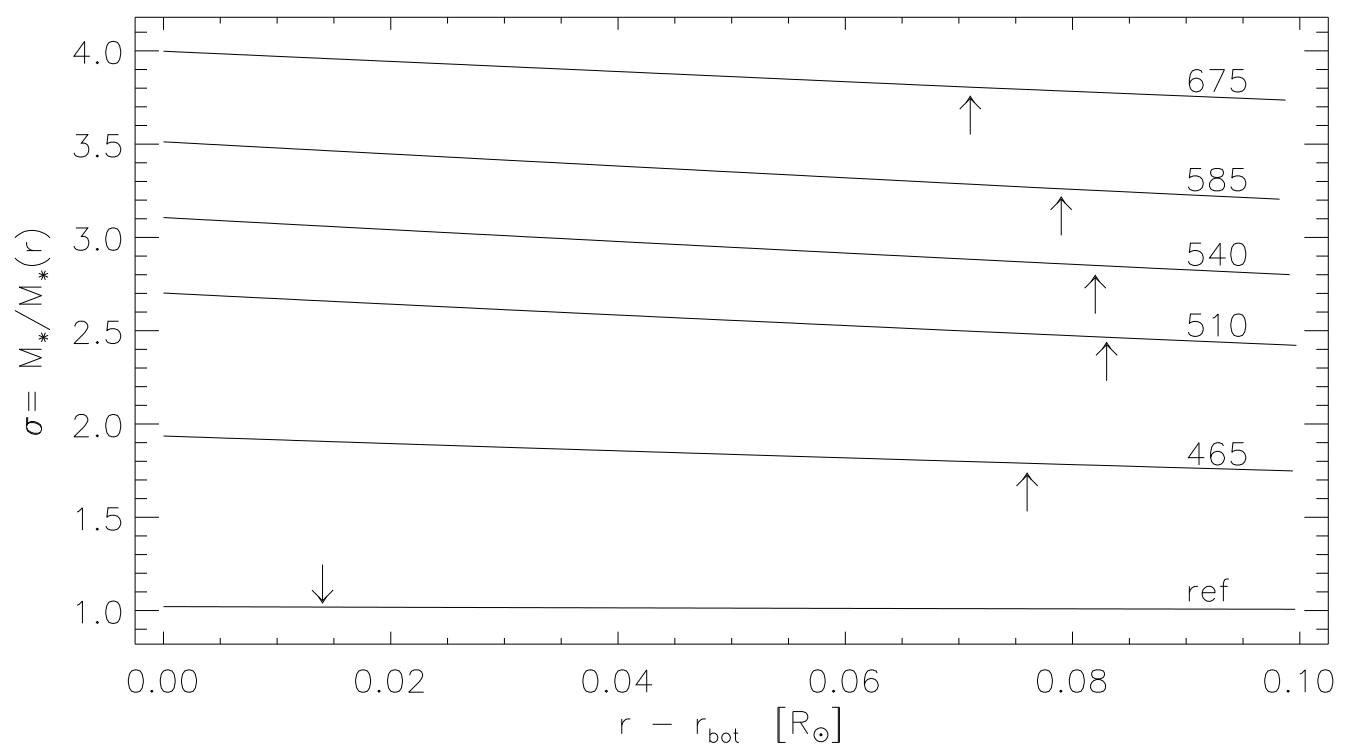

ABBILDUNG 8.4: Relative Massenverteilung $\sigma=M_{*} / M(r)$ in $1 \mathrm{M}_{\odot}$-Sterne unterschiedlicher Entwicklungsstadien. Die Nummern beziehen sich auf Sternmodelle in Tab. 4.2, 'ref' bezeichnet das Modell der heutigen Sonne. Die Abszisse gibt den Abstand über dem unteren Rand $r_{\text {bot }}$ der Overshoot-Region und die Pfeile ihre Dicke $d_{\mathrm{os}}$ an.

Die Funktion

$$
\sigma(r)=\frac{M_{*}}{M(r)} \geq 1
$$

beschreibt das Verhältnis zwischen der Gesamtmasse $M_{*}$ des Hauptsterns und der innerhalb einer Kugel mit Radius $r$ enthaltenen Masse $M(r)$. Abbildung 8.4 gibt einen Überblick über den Verlauf von $\sigma$ im Bereich der Overshoot-Region für $1 \mathrm{M}_{\odot}$-Sterne unterschiedlicher Entwicklungsstadien. Die Kurve 'ref' bezieht sich auf das Modell der heutigen Sonne, für sie gilt $\sigma \simeq 1$. In späteren Entwicklungsstadien, in denen der Stern sich zu einem (Unter-)Riesen mit einer sehr ausgedehnten äußeren Konvektionszone aufbläht, liegt ein nicht zu vernachlässigender Teil der Masse oberhalb der Overshoot-Region, weshalb der Faktor $\sigma>1$ beispielsweise bei RS CVn-Systemen berücksichtigt werden muss.

In Hinblick auf die in Abschn. 9.2 folgende Stabilitätsanalyse werden die Komponenten von $\mathbf{g}_{\text {eff }}$ in Zylinderkoordinaten (Abstand $R$ von der Rotationsachse des Hauptsterns, Azimut $\phi$, Abstand $z$ von der Äquatorebene) explizit angegeben:

$$
\begin{aligned}
\frac{\left(\mathbf{g}_{\text {eff }} \cdot \mathbf{e}_{R}\right)}{g_{*}} & =-\left(1-\epsilon^{3} \sigma\left(1+\frac{3}{2} q\right)\right) \cos \lambda+\epsilon^{3} \frac{3}{2} \sigma q \cos \lambda \cos 2 \phi \\
\frac{\left(\mathbf{g}_{\text {eff }} \cdot \mathbf{e}_{\phi}\right)}{g_{*}} & =-\epsilon^{3} \frac{3}{2} \sigma q \cos \lambda \sin 2 \phi \\
\frac{\left(\mathbf{g}_{\text {eff }} \cdot \mathbf{e}_{z}\right)}{g_{*}} & =-\left(1+\epsilon^{3} \sigma q\right) \sin \lambda
\end{aligned}
$$

Anhand der Gln. (8.13) - (8.17) und (8.10) lässt sich zusammenfassend sagen, dass die 
durch den Begleitstern hervorgerufenen Gezeiten in der hier verwendeten Näherung niedrigster Ordnung eine $\pi$-periodische Variation der Gravitation $\mathbf{g}_{\text {eff }}$ und des Potenzials $\Psi_{\text {eff }}$ in azimutaler Richtung verursachen. Im Vergleich zu einem sphärisch-symmetrischen Einzelstern ist die relative Abweichung von der Größenordnung $\mathcal{O}\left(\epsilon^{3}\right) \lesssim 10^{-2}$. Wichtiger als diese kleine quantitative Änderung ist jedoch der qualitative Unterschied, dass im Doppelsternproblem weder Kugel- noch Zylindersymmetrie besteht. Diese Brechung der Symmetrie spiegelt sich im Stabilitäts- und Ausbruchsverhalten magnetischer Flussröhren wieder: Aufgrund der dominierenden $\pi$-Periodizität des Gezeiteneinflusses in azimutaler Richtung ähnelt sich ihr Verhalten auf der dem Begleiter zugewandten bzw. abgewandten Hemisphäre des Hauptsterns.

\subsubsection{Deformation des Sternkörpers}

Wie frühere Untersuchungen im Fall der Sonne zeigen, kann die differentielle Rotation vernachlässigt werden, da sie i.Allg. keinen großen Einfluss auf die Ausbruchscharakteristiken magnetischer Flussröhren hat. Zudem ist sie aufgrund der GezeitenSynchronisation in engen Doppelsternen deutlich geringer als in der Sonne. Gleichfalls wird von meridionalen Strömungen abgesehen, da die in dem Synchronisationsmodell von Tassoul \& Tassoul (1992) postulierten, starken meridionalen Strömungen nach der Synchronisation verschwinden und auch die deutlich kleineren Geschwindigkeiten der Eddington-Sweet-Zirkulationen keine wesentliche Rolle spielen. In diesem Sinne wird im vorliegenden Modell von einer starren Rotation des Hauptsterns ausgegangen, dessen Struktur lediglich durch die Gezeitenwirkung des Begleitsterns marginal verändert wird. Da diese Deformation sehr klein ist, wird angenommen, dass sich die grundlegende Physik des hydrostatischen Sternaufbaus gegenüber einem nicht-rotierenden Einzelstern nicht nachhaltig ändert, die Schichtung also immer noch allein eine Funktion des (effektiven) Potenzials $\Psi_{\text {eff }}$ und eine Größe $f$ (z.B. die Dichte $\rho$ oder der Druck $p$ ) auf einer Äquipotenzialfläche konstant ist:

$$
f=f\left(\Psi_{\text {eff }}\right)
$$

Der Wert von $f$ am Ort $(r, \phi, \lambda)$ wird mittels einer linearen Näherung aus der Schichtung eines Einzelsterns und der Potenzialänderung $\Psi_{\text {tide }}$ bestimmt. Mit Hilfe der Gl. (8.10) erhält man die Näherung

$$
\begin{aligned}
f(r, \phi, \lambda) & =f\left(\Psi_{\text {eff }}\right)=f\left(\Psi_{*}+\Psi_{\text {tide }}\right) \\
& \approx f\left(\Psi_{*}\right)+\left.\frac{\mathrm{d} f}{\mathrm{~d} \Psi}\right|_{r} \Psi_{\text {tide }}=f\left(\Psi_{*}\right)\left(1+\frac{r}{H_{f}} \frac{\Psi_{\text {tide }} r}{G M_{*}} \sigma\right) \\
& =f(r)\left(1+\bar{r} \frac{r}{H_{f}}+\tilde{r} \frac{r}{H_{f}} \cos 2 \phi\right),
\end{aligned}
$$

mit den dimensionslosen breitenabhängigen Faktoren

$$
\bar{r}=\epsilon^{3} \frac{\sigma}{2}\left[\left(1+\frac{5}{2} q\right) \cos ^{2} \lambda-q\right] \quad \text { und } \quad \tilde{r}=\epsilon^{3} \frac{3}{4} \sigma q \cos ^{2} \lambda,
$$

und der Skalenhöhe der Größe $f$

$$
\frac{1}{H_{f}}=-\frac{\mathrm{d} \ln f}{\mathrm{~d} r}
$$


Dabei wurde in Gl. (8.19) der Zusammenhang $\mathrm{d} \Psi_{*}=-g_{*} \mathrm{~d} r=-\mathrm{d} r G M(r) / r^{2}$ verwendet. Die Größen $f(r)$ und $H_{f}(r)$ werden dem Einzelsternmodell entnommen.

Aus Gl. (8.10) erhält man mit $\Psi_{\text {eff }}=$ const. und $r=r_{0}+\delta r$ die geometrische Deformation des Sternkörpers:

$$
\frac{\delta r}{r_{0}} \approx \frac{\Psi_{\text {tide }}}{\Psi_{*}}=\bar{r}+\tilde{r} \cos 2 \phi
$$

Eine Äquipotenzialfläche wird demnach durch den Ausdruck

$$
r_{\Psi}(\phi, \lambda) \approx r_{0}(1+\bar{r}+\tilde{r} \cos 2 \phi)
$$

beschrieben. In erster Näherung ergibt sich ein entlang der Verbindungslinie $\overline{M_{*} M_{\text {co }}}$ gestrecktes Ellipsoid, das am Äquator etwas größer und an den Polen etwas kleiner als die ungestörte Kugel ist. Für die Halbachsen des Ellipsoides $d_{1} \mathbf{e}_{x}, d_{2} \mathbf{e}_{y}$ und $d_{3} \mathbf{e}_{z}$ gelten die Beziehungen

$$
\begin{aligned}
d_{1} & \simeq r_{0}\left(1+\epsilon^{3} \frac{\sigma}{2}(1+3 q)\right), \\
d_{2} & \simeq r_{0}\left(1+\epsilon^{3} \frac{\sigma}{2}\right) \\
\text { und } \quad d_{3} & \simeq r_{0}\left(1-\epsilon^{3} \frac{\sigma}{2} q\right),
\end{aligned}
$$

wobei im kartesischen Koordinatensystem die Rotation um die z-Achse $\mathbf{e}_{z}$ erfolgt und der Begleitstern in der Richtung $\mathbf{e}_{x}$ liegt. Im Fall eines mit der Periode $T$ rotierenden Einzelsterns ohne Begleiter $\left[q=0\right.$ und $\left.\epsilon^{3}=\epsilon^{3}(T)\right]$ geben diese Gleichungen die axialsymmetrische Rotationsabplattung des Sterns mit $d_{1}=d_{2}>d_{3}$ wieder.

\subsubsection{Qualität der Näherung}

Aus Gl. (8.19) ist ersichtlich, dass die Änderung einer Größe $f$ (mit $f \in[p, \rho, \ldots]$ ) aufgrund des Doppelsterncharakters von ihrer Skalenhöhe $H_{f}$ abhängt. Gleichung (8.19) kann mit Hilfe der Gl. (8.22) umgeformt werden,

$$
f(r, \phi, \lambda)=f(r)\left(1+\frac{\delta r}{H_{f}}\right) .
$$

Diese Approximation ist nur in einem Radiusintervall $r \pm H_{f}$ zulässig, in dem sich die Größe $f$ näherungsweise linear ändert. Als Grenzkriterium dient das Größenverhältnis

$$
\frac{\delta r}{H_{\min }} \lesssim 1 \quad \text { mit } \quad H_{\min }=\min _{f}\left\{H_{f}\right\}
$$

Bei einer Verletzung dieser Bedingung, z.B. aufgrund einer stärkeren Deformation in einem engeren Doppelsternsystem oder einer kleineren Skalenhöhe, ist die Näherung (8.19) unzulässig.

In Hinblick auf die Stabilitätsanalyse ist die Situation in der Overshoot-Region von besonderer Bedeutung. In ihr nimmt die Superadiabatizität $\delta=\nabla-\nabla_{\text {ad }}$ innerhalb einer 


\begin{tabular}{rll}
\multicolumn{3}{c}{ Systemparameter } \\
\hline \hline Hauptstern & $M_{*}=1 \mathrm{M}_{\odot}$, & $R_{*}=1 \mathrm{R}_{\odot}$ \\
Overshoot-Region & $r_{\text {bot }}=5.02 \cdot 10^{10} \mathrm{~cm}$, & $d_{\mathrm{os}}=10^{9} \mathrm{~cm}$ \\
Massenverhältnis & $q=1$ & \\
der Komponenten & & \\
Rotation & $T=2 \mathrm{~d}$, & $\Omega=3.64 \cdot 10^{-5} \mathrm{~Hz}$ \\
Kepler-Abstand & $a=8.41 \mathrm{R}_{\odot}$ & vgl. Gl. (8.2) \\
\hline
\end{tabular}

TABelle 8.1: Charakteristische Parameter des Referenz-Doppelsternsystems

dünnen Schicht stark ab. Bei dem im folgenden Abschnitt 8.2 beschriebenen Referenzfall ist $H_{\delta} \approx 4400 \mathrm{~km}$ und (am Äquator) $\delta r \approx 330 \mathrm{~km}$; daraus folgt $\delta r / H_{\delta} \approx 8 \cdot 10^{-2}$. Während des Aufstiegs einer Flussröhre in der Konvektionszone wächst $\delta r$ zwar etwas an, aber im Gegenzug ist die Skalenhöhe $H_{\delta}$ dort wesentlich größer. Die Bedingung (8.28) ist somit sehr gut erfüllt.

\subsection{Referenzsystem}

Zur Diskussion und Darstellung der Ergebnisse wird ein Referenzsystem verwendet, das aus zwei $1 \mathrm{M}_{\odot}$-Sternen besteht, die sich mit einer Umlaufsperiode von 2 Tagen in einem Abstand von etwa $8.4 \mathrm{R}_{\odot}$ auf Kreisbahnen um den gemeinsamen Massenschwerpunkt bewegen. Dabei steht die Untersuchung des grundlegenden Einflusses des Begleitsterns auf die Entwicklung und den Ausbruch magnetischer Flussröhren im Vordergrund, und nicht die Reproduktion beobachteter Oberflächenstrukturen eines konkreten Doppelsterns. Aus diesem Grund wird für den Aufbau des Hauptsterns von einem Modell der 'heutigen' Sonne ausgegangen, das bereits umfassend untersucht wurde und gute Vergleichsmöglichkeiten bietet. Der untere Rand der Overshoot-Region liegt hier bei $r_{\text {bot }}=0.721 \mathrm{R}_{\odot}$, ihre Dicke beträgt $d_{\mathrm{os}} \simeq 10000 \mathrm{~km}$.

Vom Standpunkt der magnetischen Aktivität von Doppelsternen aus gesehen entspricht diese Wahl des Referenzsystem - mit einem Hauptreihenstern als aktiver Komponente - mehr einem BY Dra- statt einem RS CVn-System. Allerdings geht man davon aus, dass auch die Untergruppe der kurz-periodischen RS CVn's mit Rotationsperioden $T \lesssim 1 \mathrm{~d}$ aus Hauptreihensternen bestehen. 


\section{Kapitel 9}

\section{Gleichgewicht und Stabilität magnetischer Flussröhren in engen Doppelsternsystemen}

\subsection{Stationäres Gleichgewicht}

In Anlehnung an das Einzelsternproblem (Abschn. 2.2) werden die Eigenschaften von auftriebsneutralen Flussröhren im stationären mechanischen Gleichgewicht innerhalb der Overshoot-Region untersucht. Aufgrund der Auftriebsneutralität ist die Bewegungsgleichung unabhängig von der effektiven Gravitation $\mathbf{g}_{\text {eff }}$. Wie in Abschn. 2.2 beschrieben, müssen Flussröhren in diesem Fall senkrecht zur Rotationsachse in einer Ebene parallel zum Äquator des Sterns bzw. zur Orbitalebene des Systems liegen. Auch in einem Doppelstern haben sie die Form eines Torus mit konstanter Krümmung:

$$
\kappa=\frac{1}{R}=\text { const. }
$$

Dabei bezeichnet $R$ den Krümmungsradius. Die Gleichgewichtsbeziehung zwischen der relativen Strömungsgeschwindigkeit $v$ entlang der Flussröhre, der Dichte $\rho$ und dem Magnetfeld $B$ ist fast identisch mit der Bedingung (2.38). Im Unterschied zum Einzelsternproblem ist die Dichte nun jedoch entlang der Flussröhre nicht konstant. In dem leicht deformierten Stern weicht die Form der Äquipotenzialflächen, d.h. der Flächen, auf denen eine Größe der Sternschichtung konstant ist, von der sphärischen Form ab (vgl. Abschn. 8.1). Aus diesem Grund schneidet bzw. berührt eine ringförmige Flussröhre verschiedene Äquipotenzialflächen, wodurch es zu einer periodischen Variation der Dichte $\rho\left(\equiv \rho_{\mathrm{e}}\right)$ mit dem Azimutwinkel $\phi$ entlang des Ringes kommt (Abb. 9.1). Mit Hilfe der Näherung (8.19) für die Dichte $\rho$ am Ort $(r, \phi, \lambda)$,

$$
\frac{\rho}{\rho_{0}}=1+\bar{r} \frac{r}{H_{\rho, \mathrm{e}}}+\tilde{r} \frac{r}{H_{\rho, \mathrm{e}}} \cos 2 \phi,
$$

erhält man für die relative Variation des Magnetfelds und der Strömungsgeschwindigkeit die Ausdrücke

$$
\frac{B}{B_{0}}=\frac{1-M_{\alpha, 0}^{2}}{\left(\frac{\rho}{\rho_{0}}\right)-M_{\alpha, 0}^{2}}\left(\frac{\rho}{\rho_{0}}\right)
$$




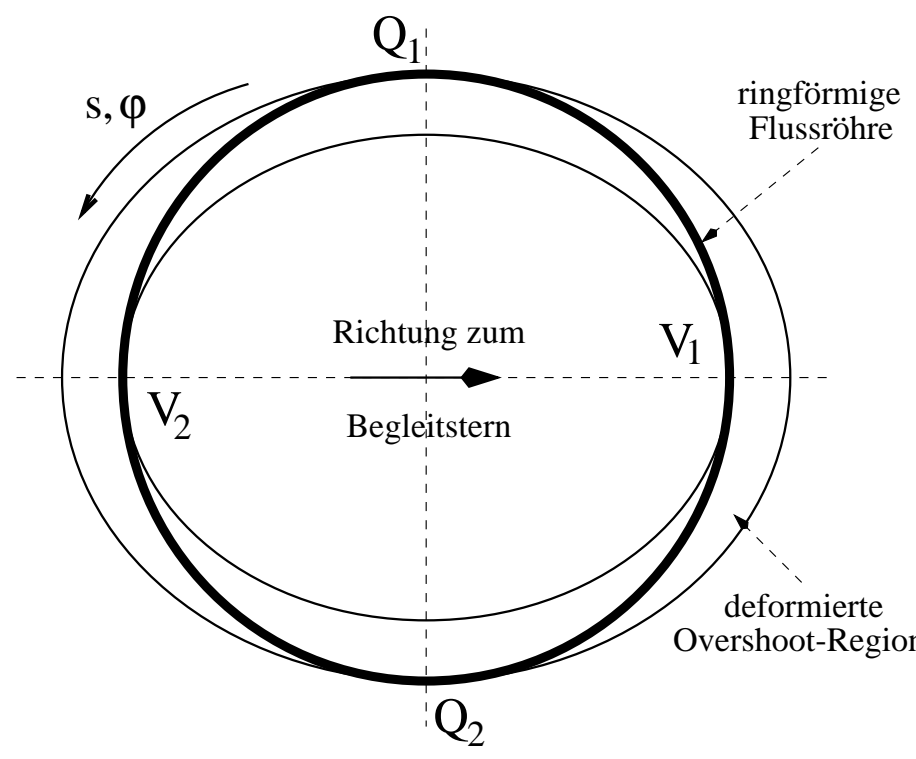

AbBILDUNG 9.1: Toroidale Flussröhre im stationären mechanischen Gleichgewicht in einem Doppelstern. Aufgrund der (hier stark übertriebenen) Deformation des Sterns liegen einzelne Segmente des Flussrings auf verschiedenen Äquipotenzialflächen; die Umgebungsbedingungen an den Punkten $V_{1,2}$ entsprechen denen einer tiefer im Sterninnern liegenden Schicht als die der Quadraturpunkte $Q_{1,2}$.

und

$$
\frac{v}{v_{0}}=\frac{1-M_{\alpha, 0}^{2}}{\left(\frac{\rho}{\rho_{0}}\right)-M_{\alpha, 0}^{2}} .
$$

Dabei sind $v_{0}, B_{0}, \rho_{0}$ und $M_{\alpha, 0}$ die Werte einer vergleichbaren Flussröhre in einem Einzelstern, die durch die Gleichgewichtsbedingung (2.38) und die Beziehung (2.39) miteinander verknüpft sind.

Für die Struktur einer Flussröhre im mechanischen Gleichgewicht ergibt sich somit folgendes Bild: Bei den Punkten $V_{1,2}$, d.h. in der Nähe der Verbindungslinie, ist die Dichte etwas größer als an den Quadraturpunkten $Q_{1,2}$, da diese Teile in eine Umgebung eingebettet sind, die einer etwas tiefer liegenden Schicht im Stern entspricht (vgl. Abb. 9.1). Entsprechend der Beziehungen (9.3) ist das Magnetfeld hier kleiner und der Flussröhrenquerschnitt entsprechend größer als an den Quadraturpunkten. Aufgrund des konstanten Massenfluss entlang der Röhre ist die interne Strömungsgeschwindigkeit bei $V_{1,2}$ minimal.

Die stationären Bewegungsgleichungen werden bei diesem mechanischen Gleichgewicht korrekt erfüllt. Die Erhaltung der Energie führt dabei aber zu Bedingungen, die unter adiabatischen Verhältnissen, wie sie für die Flussröhrenspeicherung aufgrund der langen thermischen Austauschzeiten angenommen werden müssen, nicht ganz realistisch erscheinen. Durch die Auftriebsneutralität ist die Variation des Magnetfelds (9.3) als Funktion der Dichte gegeben.

Mit dem lateralen Druckgleichgewicht (2.26) existiert aber eine weitere Beziehung zwischen Magnetfeld und internem Gasdruck ${ }^{1}$. Die Kombination dieser beiden Beziehungen zeigt, dass das Gas im Inneren der Flussröhre nicht isentrop ist und die Entropie $S$ sich mit dem Azimutwinkel $\phi$ periodisch ändert:

$$
\frac{\partial S}{\partial \phi}=c_{p} \frac{\mathrm{d} \ln p_{\mathrm{e}}}{\mathrm{d} \phi} \frac{1}{\beta}\left[\beta \delta+\frac{1}{\gamma}\left(1+\frac{2 M_{\alpha}{ }^{2}}{1-M_{\alpha}{ }^{2}}(1-\nabla)\right)\right]
$$

\footnotetext{
${ }^{1}$ Der externe Gasdruck wird durch die Gleichgewichtslage der toroidalen Flussröhre bestimmt.
} 
Der Term in eckigen Klammern ist für die hier relevanten Parameterbereiche von der Größenordnung $\lesssim 1$. Mit der Näherung (8.19) für die Variation des externen Drucks folgt

$$
\frac{1}{c_{p}} \frac{\partial S}{\partial \phi} \sim \frac{1}{\beta} \frac{\mathrm{d} \ln p_{\mathrm{e}}}{\mathrm{d} \phi} \sim \frac{\epsilon^{3}}{\beta} \frac{r}{H_{\mathrm{p}, \mathrm{e}}} .
$$

Für das Skalenverhältnis gilt zwar $r / H_{p, \mathrm{e}} \simeq 9$, aber da die Stabilitätsanalyse bei Werten $\beta \approx 10^{5} \ldots 10^{6} \gg 1$ durchgeführt wird, ist die relative Entropievariation entlang der Flussröhre um einige Größenordnungen kleiner als die $\epsilon$-Terme höherer Ordnung, die bei den Näherungen (8.10), (8.13) und (8.19) vernachlässigt wurden. Aufgrund der internen Strömung $v$ muss sich die Entropie eines Gaselements in Abhängigkeit von der momentanen Orientierung zum Begleitstern mit der Zeit periodisch ändern. Ein physikalischer Mechanismus, der diese Entropieänderung bewirken könnte ist aber nicht bekannt. Andererseits ist die charakteristische Zeitskala $\Delta t_{S}$, auf der sich diese Änderung abspielen müsste, von der Ordnung

$$
\Delta t_{S} \simeq \frac{1}{2} \frac{\pi R}{v}=\frac{T}{4} \frac{1}{R o}=\frac{T}{8}\left(\frac{1}{M_{\alpha}^{2}}-1\right) .
$$

In schnell rotierenden Doppelsternen mit $R o \ll 1$ bzw. für $M_{\alpha} \ll 1$ ist diese Zeitskala deutlich länger als die üblichen Anwachszeiten der Parker-Instabilität von einigen hundert Tagen. Die Entropievariation ist damit für die relevanten Konfigurationen praktisch unerheblich. Die Situation wird zusätzlich dadurch deutlich vereinfacht, dass die Stabilität wesentlich stärker von der externen Schichtung abhängt als vom internen Dichteverlauf. Untersuchungen magnetischer Flussröhren in Einzelsternen (Spruit \& van Ballegooijen 1982, Ferriz-Mas \& Schüssler 1993, 1995) zeigen, dass die Stabilität im Wesentlichen durch die Superadiabatizität $\delta$ in der Overshoot-Region bestimmt wird. Aufgrund der im Vergleich zur Dichteskalenhöhe $H_{\rho}$, die in Gl. (9.2) auftritt, viel kleineren Skalenhöhe $H_{\delta}$, wird die $\phi$-Variation im Doppelstern durch die Winkelabhängigkeit von $\delta$ dominiert. Dieser Punkt wird durch den Vergleich der beiden Größen $\rho$ und $\delta$ in Abb. 9.2 verdeutlicht. Der Einfluss des Begleitsterns auf die Stabilität der Flussröhre wird fast ausschließlich durch die Variation der Superadiabatizität,

$$
\delta(\phi)=\delta(r)\left(1+\frac{\delta r}{H_{\delta}}\right),
$$

vermittelt, sodass die interne Dichte- und die dadurch entstehende Entropievariationen entlang der Flussröhre vernachlässigbar sind.

\subsubsection{Referenzgleichgewicht}

Die Ausgangslage einer magnetischen Flussröhre im stationären Gleichgewicht sei im Referenzfall die Mitte der Overshoot-Region des Hauptsterns des in Abschn. 8.2 beschriebenen Doppelsternsystems, für die in Tab. 9.1 einige charakteristische Größen der Sternschichtung zusammengefasst sind. Zusätzlich werden einige Parameter angegeben, die sich aus der analytischen Beschreibung des Doppelsterncharakters in Abschn. 8.1.2 ergeben. Der magnetische Fluss (2.27) ergibt sich aus dem Durchmesser des Röhrenquerschnitts $d$, für den hier $2000 \mathrm{~km}$ angenommen wird, und der Magnetfeldstärke $B$, deren Wert entsprechend den Ergebnissen der Stabilitätsanalyse gewählt wird. 

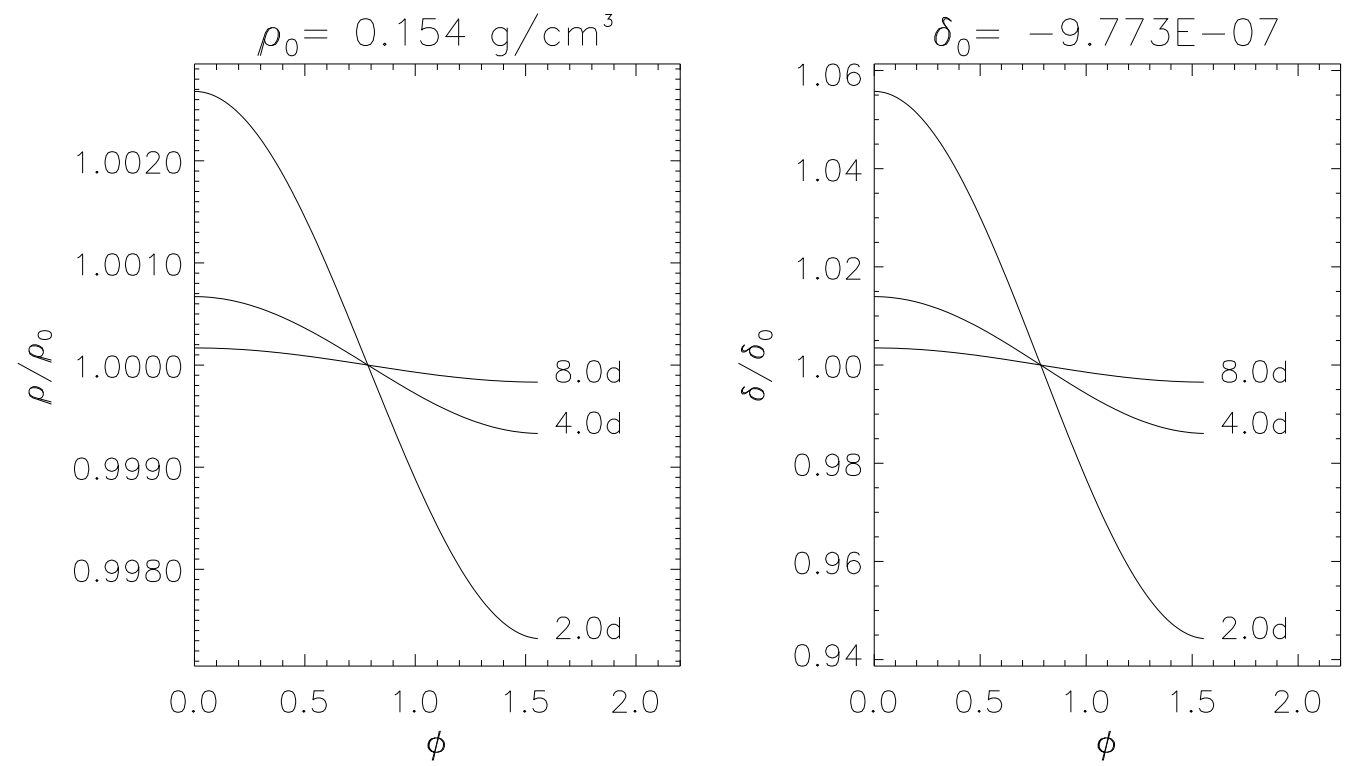

ABBILDUNG 9.2: Vergleich zwischen der Variation der Dichte $\rho$ und der Superadiabatizität $\delta$ für Doppelsternsysteme mit $M_{*}=1 \mathrm{M}_{\odot}$ und $q=1$ bei verschiedenen Rotationsperioden. Aufgrund der wesentlich kleineren Skalenhöhe $H_{\delta}$ ist die resultierende Variation der Superadiabatizität deutlich größer als die der Dichte; im hier dargestellten Referenzfall (Abschn. 9.1.1) ist $H_{\rho} \simeq 21 H_{\delta}$.

\section{Sternschichtungs- und Deformationsparameter}

\begin{tabular}{rll}
\hline \hline Tiefe & $r_{0}=5.07 \cdot 10^{10} \mathrm{~cm}=0.73 \mathrm{R}_{\odot}$ & \\
ex. Gasdruck & $p_{\mathrm{e} 0}=4.31 \cdot 10^{13} \mathrm{dyn} / \mathrm{cm}^{2}$, & $H_{\mathrm{p}, \mathrm{e}}=5.52 \cdot 10^{9} \mathrm{~cm}$ \\
ex. Dichte & $\rho_{\mathrm{e} 0}=0.15 \mathrm{~g} / \mathrm{cm}^{3}$, & $H_{\rho, \mathrm{e}}=9.21 \cdot 10^{9} \mathrm{~cm}$ \\
Superadiabatizität & $\delta=-9.77 \cdot 10^{-7}$, & $H_{\delta}=4.43 \cdot 10^{8} \mathrm{~cm}$ \\
\hline Abstandsverhältnis & $\epsilon^{3}=6.53 \cdot 10^{-4}$ & vgl. Gl. (8.11) \\
Massenkorrektur & $\sigma \simeq 1$ & vgl. Gl. (8.14) \\
\hline Deformation am & $\bar{r}=8.16 \cdot 10^{-4}$ & vgl. Gl. (8.20) \\
Äquator $(\lambda=0)$ & $\tilde{r}=4.90 \cdot 10^{-4}$ & \\
\hline
\end{tabular}

TABELle 9.1: Charakteristische Größen am Ort des Referenz-Gleichgewichts 


\subsection{Lineare Stabilitätsanalyse}

Gegenstand der folgenden Stabilitätsanalyse ist eine auftriebsneutrale toroidale Flussröhre im stationären Kräftegleichgewicht innerhalb der Overshoot-Region eines Doppelsterns. Es handelt sich dabei um eine Erweiterung der Arbeiten von Ferriz-Mas \& Schüssler (1993, 1995), in denen das analoge Einzelsternproblem behandelt wird. Die Erweiterung auf Doppelsterne berücksichtigt die durch den Begleitstern hervorgerufene Deformation des Hauptsterns sowie die periodische Änderung der Gezeitenkraft entlang des Flussrings (Holzwarth \& Schüssler 2000).

\subsubsection{Mathematische Formulierung}

Der Vektor $\xi(\phi, t)=\left(\xi_{\mathrm{t}}, \xi_{\mathrm{n}}, \xi_{\mathrm{b}}\right)^{T}$ beschreibe die Auslenkung eines Röhrenelements aus der toroidalen Gleichgewichtslage der Flussröhre im Koordinatensystem des lokalen Dreibeins (2.17); in Zylinderkoordinaten gilt für einen parallel zum Äquator liegenden Flussring

$$
\mathbf{t}_{0}=\mathbf{e}_{\phi} \quad, \quad \mathbf{n}_{0}=-\mathbf{e}_{R} \quad \text { und } \quad \mathbf{b}_{0}=\mathbf{e}_{z} .
$$

Die Zeitabhängigkeit der Auslenkung wird mit dem Exponential-Ansatz

$$
\xi(\phi, t) \sim \exp (i \omega t)
$$

absepariert, wobei $\omega$ die (komplexe) Eigenfrequenz ist. Deren Realteil $\Re(\omega)$ beschreibt die oszillatorische Zeitabhängigkeit der Lösung und der Imaginärteil die Anwachsrate: Für $\Im(\omega)=0$ ist die Störung stabil, für $\Im(\omega)>0$ zeitlich abklingend und für $\Im(\omega)<0$ anwachsend. Die charakteristische Zeitskala einer anwachsenden bzw. abklingenden Störung ist $\tau=1 /|\Im(\omega)|$. Für die numerische Behandlung erweist es sich als vorteilhaft, die Eigenfrequenz $\omega$ mit der konstanten Rotationsfrequenz $\Omega$ des Sterns (bzw. Doppelsternsystems) zu normieren:

$$
\tilde{\omega}=\frac{\omega}{\Omega}
$$

Damit erhält man die linearisierten Bewegungsgleichungen eines Flussrings bei adiabatischen Störungen in ihrer Lagrangeschen Form:

$$
\xi^{\prime \prime}+\left(\mathcal{M}_{\phi}+i \tilde{\omega} \mathcal{M}_{\phi t}\right) \xi^{\prime}+\left(\mathcal{M}_{\xi}+i \tilde{\omega} \mathcal{M}_{t}-\tilde{\omega}^{2} \mathcal{M}_{t t}\right) \xi=0
$$

Striche ' bezeichnen die Ortsableitung nach dem Azimutwinkel $\phi$. Die von $\phi$ abhängigen Koeffizientenmatrizen $\mathcal{M}$, die im Anhang A explizit angegeben sind, enthalten einen $\pi$ periodischen Anteil, der mittels der allgemeinen Schreibweise

$$
\mathcal{M}(\phi)=\mathcal{M}_{0}+\epsilon^{3} \mathcal{M}_{\mathrm{c}} \cos 2 \phi+\epsilon^{3} \mathcal{M}_{\mathrm{s}} \sin 2 \phi=\mathcal{M}(\phi+\pi)
$$

in einen symmetrischen und unsymmetrischen Beitrag bezüglich der Verbindungslinie $\overline{M_{*} M_{\mathrm{co}}}$ aufgespalten wird. Die Koeffizientmatrizen $\mathcal{M}_{\mathrm{c} / \mathrm{s}}$ erhält man durch Substitution aller Gleichgewichtsgrößen mit den Näherungen (8.19) bzw. (8.15) - (8.17) und anschließender Zusammenfassung gleichartiger Terme; der Sinusterm entsteht durch die azimutale Komponente $g_{\phi}$ der Gezeitenkraft [vgl. Gl. (8.16)]. 
Wegen der Geschlossenheit der Flussröhre muss eine Eigenfunktion des Problems die Bedingungen

$$
\xi(\phi+2 \pi)=\xi(\phi) \quad \text { und } \quad \xi^{\prime}(\phi+2 \pi)=\xi^{\prime}(\phi)
$$

erfüllen. Für den ortsabhängigen Teil der Eigenfunktion wird deshalb der Ansatz

$$
\xi(\phi)=\hat{\xi}(\phi) e^{i m \phi}=\left(\sum_{k=-\infty}^{\infty} \hat{\xi}_{k} e^{i k \phi}\right) e^{i m \phi}
$$

verwendet; in Analogie zum Einzelsternproblem wird ein Phasenfaktor mit der azimutalen Wellenzahl $m$ abgespalten. Die Amplitudenfunktion $\hat{\xi}(\phi)$ ist aufgrund der Bedingungen (9.14) eine $2 \pi$-periodische Funktion. Die Amplituden $\hat{\xi}_{k}$ der Partialwellen ergeben sich für festes $m$ aus den 3-Term-Rekursionsgleichungen

$$
\mathcal{L}_{m+k} \hat{\xi}_{k-2}+\mathcal{C}_{m+k} \hat{\xi}_{k}+\mathcal{R}_{m+k} \hat{\xi}_{k+2}=0 \quad, \quad \forall k
$$

bzw. aus dem zweiseitig-unendlichen Hillschen Gleichungssystem

$$
\underbrace{\left(\begin{array}{ccccccc}
\ddots & \ddots & \ddots & & & & \\
0 & \mathcal{L}_{m-2} & \mathcal{C}_{m-2} & \mathcal{R}_{m-2} & 0 & & \\
& 0 & \mathcal{L}_{m} & \mathcal{C}_{m} & \mathcal{R}_{m} & 0 & \\
& & 0 & \mathcal{L}_{m+2} & \mathcal{C}_{m+2} & \mathcal{R}_{m+2} & 0 \\
& & & \ddots & \ddots & \ddots
\end{array}\right)}_{\text {Hillmatrix } \mathcal{H}}\left(\begin{array}{c}
\vdots \\
\hat{\xi}_{-2} \\
\hat{\xi}_{0} \\
\hat{\xi}_{2} \\
\vdots
\end{array}\right)=0 .
$$

Für eine ausführliche Herleitung dieser Gleichungen wird z.B. auf Morse \& Feshbach (1953) oder Abramowitz \& Stegun (1972) verwiesen. Die Matrizen $\mathcal{L}$ und $\mathcal{R}$ (siehe Anhang A) haben ihren Ursprung in der periodischen Variation des Gleichgewichts. Im Einzelsternproblem verschwinden diese Matrizen und das Problem reduziert sich auf die Lösung der homogenen Gleichungssysteme ${ }^{2} \mathcal{C}_{m} \hat{\xi}_{0}=0, \forall m$. Im Doppelsternproblem existiert hingegen eine Kopplung zwischen einem Wellenmodus $m$ und den 'benachbarten' Wellenmodi $m \pm 2$, die durch die beiden Nebendiagonalbänder der Hillschen Matrix vermittelt wird und die Abhängigkeit der Stabilität vom Azimut $\phi$ bewirkt. Ein Eigenmodus besteht demzufolge aus einem Spektrum von Partialwellen mit den jeweiligen Amplituden $\hat{\xi}_{k}(k \rightarrow \pm \infty)$, weshalb es im Grunde nicht zulässig ist, von einem Eigenmodus der Wellenzahl $m$ zu sprechen. Da die Matrizen $\mathcal{L}$ und $\mathcal{R}$ im Vergleich zu $\mathcal{C}$ aber relativ klein sind, ist die Kopplung zwischen den benachbarten Partialwellen nur gering. Der Eigenmodus wird deshalb durch die Welle $\hat{\xi}_{0}$ dominiert und kann in diesem Sinne auch weiterhin durch die dazugehörige Wellenzahl $m$ klassifiziert werden.

Um einen ersten Anhaltspunkt bezüglich der resultierenden Struktur der Eigenfunktionen zu erhalten, werden für die folgende Betrachtung Partialwellen mit $|k|>2$ vernachlässigt; aufgrund der geringen Kopplung ist diese Näherung zulässig. Die Komponenten $\xi_{\mathrm{x}}$ mit $\mathrm{x} \in[\mathrm{t}, \mathrm{n}, \mathrm{b}]$ haben nach dem Ansatz (9.15) die Form

$$
\xi_{\mathrm{x}}(\phi, t)=\left|\hat{\xi}_{\mathrm{x}}(\phi)\right| \exp \left[i\left(\arg \hat{\xi}_{\mathrm{x}}(\phi)+m \phi+\omega t\right)\right] .
$$

\footnotetext{
${ }^{2}$ Die Determinante von $\mathcal{C}_{m}$ ist identisch mit der Dispersionsgleichung (2.43) für die Wellenzahl $m$.
} 
Dabei ist

$$
\begin{aligned}
\left|\hat{\xi}_{\mathrm{x}}(\phi)\right| & =\left|\hat{\xi}_{\mathrm{x}, 0}+\hat{\xi}_{\mathrm{x}, 2} e^{i 2 \phi}+\hat{\xi}_{\mathrm{x},-2} e^{-i 2 \phi}+\ldots\right| \\
& \simeq 1+\left|\hat{\xi}_{\mathrm{x}, 2}\right| \cos \left(2 \phi+\alpha_{2}\right)+\left|\hat{\xi}_{\mathrm{x},-2}\right| \cos \left(2 \phi-\alpha_{-2}\right) .
\end{aligned}
$$

Terme der Ordnung $\mathcal{O}\left(\left|\hat{\xi}_{\mathrm{x}, \pm 2}\right|^{2}\right)$ und höher wurden im letzten Schritt dieser Gleichung vernachlässigt, weil die Eigenfunktion durch die $\hat{\xi}_{\mathrm{x}, 0}$-Welle dominiert wird, d.h. es ist $\hat{\xi}_{\mathrm{x}, \pm 2} \ll \hat{\xi}_{\mathrm{x}, 0}:=1$. Die Argumente $\alpha_{ \pm 2}:=\arg \hat{\xi}_{\mathrm{x}, \pm 2}$ der Amplituden bewirken eine Phasenverschiebung benachbarter Partialwellen relativ zur dominierenden Welle mit der Wellenzahl $m$. Gleichung (9.19) beschreibt die resultierende Ortsabhängigkeit der Enveloppe $\left|\hat{\xi}_{\mathrm{x}}(\phi)\right|$, d.h. der einhüllenden Kurve der Auslenkung. Im vorliegenden Fall ist sie im Gegensatz zum Einzelsternproblem nicht konstant, sondern in erster Näherung eine $\pi$-periodische Funktion des Azimuts $\phi$. Die Wechselwirkung zwischen den beteiligten Partialwellen führt für $\alpha_{ \pm 2} \neq 0$ zu einer Phasenverschiebung der Extrema relativ zur Orientierung der Verbindungslinie $\overline{M_{*} M_{\mathrm{co}}}$. Die Lage $\phi_{\mathrm{ext}}$ der Extrema ergibt sich aus den Nullstellen der Ortsableitung von Gl. (9.19):

$$
\tan 2 \phi_{\mathrm{ext}}=\frac{\left|\hat{\xi}_{\mathrm{x},-2}\right| \sin \alpha_{-2}-\left|\hat{\xi}_{\mathrm{x}, 2}\right| \sin \alpha_{2}}{\left|\hat{\xi}_{\mathrm{x},-2}\right| \cos \alpha_{-2}+\left|\hat{\xi}_{\mathrm{x}, 2}\right| \cos \alpha_{2}}
$$

Diese Näherung ist nur gültig für $\hat{\xi}_{\mathrm{x}, \pm 2} \ll \hat{\xi}_{\mathrm{x}, 0}$.

Von besonderem Interesse ist die Auslenkung der Flussröhre in sphärisch-radialer Richtung $\xi_{r}$. Wir gehen von der naheliegenden Annahme aus, dass die Wahrscheinlichkeit der Bildung einer instabilen Flussröhrenschleife von dieser Komponente der Auslenkung abhängt: Je weiter ein Teil der Flussröhre in Richtung der Konvektionszone verschoben wird, desto instabiler ist die Schichtung in dessen neuen Umgebung und desto wahrscheinlicher ist sein weiterer Aufstieg zur Oberfläche aufgrund des magnetischen Auftriebs. In diesem Sinne dient die Enveloppe $\left|\hat{\xi}_{r}(\phi)\right|$ als Maß für die Wahrscheinlichkeit der Bildung einer aufsteigenden Flussröhrenschleife und gibt Hinweise auf die Existenz und Orientierung von Vorzugslängen am unteren Rand der Konvektionszone.

Der Phasenfaktor in Gl. (9.18) enthält die Zeitabhängigkeit der Störung und führt für $\Re(\omega) \neq 0$ zu einer umlaufenden Welle. Neben der Maximalauslenkung ist auch deren Ausbreitungsgeschwindigkeit ortsabhängig: Aus dem Phasenfaktor in Gl. (9.18) ergibt sich für die Phasengeschwindigkeit

$$
v_{\mathrm{p}}=\underbrace{-\frac{\Re(\omega)}{m \kappa}}_{v_{\mathrm{p}, 0}}\left(1+\frac{1}{m} \frac{d}{d \phi} \arg \hat{\xi}_{\mathrm{x}}(\phi)\right)^{-1} .
$$

Sie hängt von der Winkelabhängigkeit des Arguments $\arg \hat{\xi}_{\mathrm{x}}(\phi)$ der Amplitude ab; im Einzelsternproblem ist dieser Faktor konstant und es gilt $v_{\mathrm{p}}=v_{\mathrm{p}, 0}$. In Abb. 9.3 ist in einem Polardiagramm die Struktur eines instabilen Eigenmodus schematisch dargestellt. Die Pfeile an der äußeren und inneren Enveloppe deuten das exponentielle Anwachsen des Betrags der Auslenkung der instabilen $m=1$-Störung mit der charakteristischen 


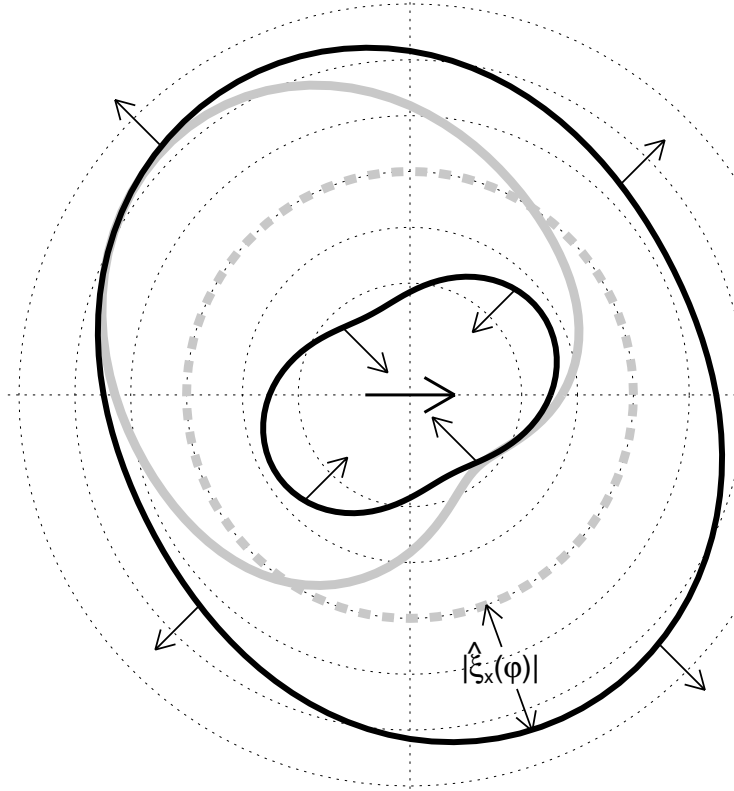

ABBildung 9.3: Polardiagramm eines instabilen Eigenmodus. Graue Linien zeigen die Flussröhre im stationären Gleichgewicht (gestrichelt) bzw. bei einer Momentaufnahme der umlaufenden Welle (durchgezogen). $\mathrm{Zu}$ jeder Zeit berührt sie die äußere und innere Enveloppe (schwarz), deren Betrag $\left|\hat{\xi}_{\mathrm{x}}(\phi)\right|$ mit der Anwachszeit $\tau$ exponentiell anwächst. Die Enveloppe zeigt einen $\pi$-periodischen Verlauf, ihre Extrema sind zur Richtung des Begleitsterns (zentraler Pfeil) phasenverschoben.

Zeitskala $\tau=1 /|\Im(\omega)|$ an. Zu beachten ist die Winkelabhängigkeit der Enveloppe und die Phasenverschiebung ihrer Maxima bezüglich der Richtung zum Begleitstern.

Das Stabilitätsproblem von Flussringen in Doppelsternen wird mit Hilfe der Theorien von Hill $^{3}$ und Floquet ${ }^{4}$ sowohl numerisch als auch - im folgenden, einfachen Sonderfall analytisch behandelt. Für eine detailliertere Diskussion der mathematischen Grundlagen wird auf die Literatur verwiesen (z.B. Collatz 1970, Yakubovich \& Starzhinskii 1975, Walter 1996).

\subsection{Flussröhren in der Äquatorebene}

Bei magnetischen Flussringen in der Äquatorebene ist die Binormalenkomponente $\xi_{\mathrm{b}}$ einer Störung von der Tangential- und Hauptnormalenkomponente entkoppelt, d.h. Auslenkungen senkrecht zur Äquatorebene sind unabhängig von Auslenkungen innerhalb dieser Ebene. Die Eigenmodi $\xi_{\mathrm{b}}(\phi, t)$ sind in diesem Sonderfall alle stabil und haben für die Entwicklung ausbrechender Flussröhren keine Bedeutung. Dennoch ist eine separate Behandlung dieses eindimensionalen Problems von Interesse, da für dessen Lösung analytische Näherungen angegeben werden können, die einen Einblick in die charakteristischen Stabilitätseigenschaften von Flussröhren auch außerhalb der Äquatorebene erlauben (Holzwarth \& Schüssler 2000).

Binormale Störungen in der Äquatorebene werden als Sonderfall der Gl. (9.12) durch die gedämpfte Matthieusche Differentialgleichung

$$
\xi_{\mathrm{b}}^{\prime \prime}-i \tilde{\omega} \xi_{\mathrm{b}}{ }^{\prime}-\tilde{\omega}^{2}(\bar{c}+\tilde{c} \cos 2 \phi) \xi_{\mathrm{b}}=0
$$

${ }^{3}$ George William Hill, amerikanischer Mathematiker, 1838 - 1914

${ }^{4}$ Gaston Floquet, französischer Mathematiker, 1847 - 1920 
beschrieben. Dazu wurden die Abkürzungen

$$
\bar{c}=\frac{1}{4 M_{\alpha 0}^{2}}\left(M_{\alpha_{0}}^{2}-1-\bar{r} \frac{r}{H_{\rho}}\right) \quad \text { und } \quad \tilde{c}=-\frac{1}{4 M_{\alpha 0}^{2}} \tilde{r} \frac{r}{H_{\rho}}
$$

verwendet; es ist $\tilde{c} \ll \bar{c}$. Die Abhängigkeit von der Dichteskalenhöhe $H_{\rho}$ taucht aufgrund des funktionalen Zusammenhangs

$$
M_{\alpha 0}=\frac{v_{0}}{c_{a, 0}}=\frac{v_{0}}{B_{0}} \sqrt{4 \pi \rho_{0}}=\frac{v_{0} \rho_{0} A_{0}}{B_{0} A_{0}} \sqrt{\frac{4 \pi}{\rho_{0}}}=\frac{\Phi_{\rho}}{\Phi} \sqrt{\frac{4 \pi}{\rho_{0}}} \propto \rho^{-1 / 2}
$$

auf, da wegen der Konstanz des magnetischen Flusses $\Phi$ und - im stationären Gleichgewicht — des Massenflusses $\Phi_{\rho}=\rho_{0} v_{0} A_{0}$ in diesem Sonderfall $\rho$ die einzige variierende Gleichgewichtsgröße ist. Die Eigenfunktion $\xi_{\mathrm{b}}(\phi)$ besteht aus einer Superposition unendlich vieler Partialwellen, deren Amplituden $\hat{\xi}_{k}$ durch das Gleichungssystem (9.16) bzw. (9.17) bestimmt werden. Für die dominierende Partialwelle $k=0$ eines Eigenmodus mit der Wellenzahl $m$ hat die Rekursionsgleichung (9.16) die explizite Form

$$
\bar{c}\left[1+f_{\epsilon}\right] \tilde{\omega}^{2}-m \tilde{\omega}+m^{2}=0 \quad \text { mit } \quad f_{\epsilon}=\frac{\tilde{c}}{2 \bar{c}}\left(\frac{\hat{\xi}_{\mathrm{b}, 2}}{\hat{\xi}_{\mathrm{b}, 0}}+\frac{\hat{\xi}_{\mathrm{b},-2}}{\hat{\xi}_{\mathrm{b}, 0}}\right) .
$$

Die Abhängigkeit vom Doppelsterncharakter $(\propto \tilde{c})$ und die Kopplung an die benachbarten Wellenmodi $\left(\propto \hat{\xi}_{\mathrm{b}, \pm 2}\right)$ sind in dem Term $f_{\epsilon}$ zusammengefasst. Die Amplitudenverhältnisse $\hat{\xi}_{\mathrm{b}, \pm 2} / \hat{\xi}_{\mathrm{b}, 0}$ hängen ihrerseits rekursiv von den Partialwellen $\hat{\xi}_{\mathrm{b}, \pm 4}, \hat{\xi}_{\mathrm{b}, \pm 6}, \ldots$ und der Eigenfrequenz $\tilde{\omega}$ selbst ab, sodass das Problem nur durch ein Iterations- oder Näherungsverfahren gelöst werden kann.

\subsubsection{Einzelsternproblem}

Der Fall $\tilde{c}=0$ entspricht der Stabilitätsanalyse einer Flussröhre in einem rotierenden Einzelstern. Die Eigenfunktionen sind harmonische Wellen $\left(\hat{\xi}_{\mathrm{b}, 0}=\right.$ const. und $\hat{\xi}_{\mathrm{b}, k}=$ $0, \forall k \neq 0$ ) mit den Eigenfrequenzen

$$
\tilde{\omega}_{(m, \bar{r}, \pm)}=m \frac{2 M_{\alpha 0}}{M_{\alpha 0} \pm \sqrt{1+\bar{r} \frac{r}{H_{\rho}}}} .
$$

Die Eigenfrequenzen $\tilde{\omega}_{(m, \bar{r},+)}$ mit dem positiven Vorzeichen im Nenner führen zu retrograden Wellenbewegungen (entgegen der Rotationsrichtung des Sterns) und die mit negativem Vorzeichen $\left(\tilde{\omega}_{(m, \bar{r},-)}\right)$ entsprechend zu prograden. Die Eigenfrequenzen höherer Wellenmodi sind Vielfache der Basisfrequenzen $\tilde{\omega}_{(1, \bar{r}, \pm)}$.

Die Abplattung des rotierenden Sternkörpers $(\propto \bar{r})$ bewirkt eine kleine Frequenzverschiebung im Vergleich zu einem kugelsymmetrischen Sternmodell, da die Schichtung am Gleichgewichtsort des Flussrings aufgrund der axialsymmetrischen Verformung formal verändert ist. Für die in Abschn. 9.1.1 beschriebene Referenz-Flussröhre ist dieser Effekt von der Größenordnung

$$
\left.\frac{\Delta \tilde{\omega}}{\tilde{\omega}}\right|_{\text {Rot }}=\frac{\tilde{\omega}_{(1, \bar{r}, \pm)}-\tilde{\omega}_{(1,0, \pm)}}{\tilde{\omega}_{(1,0, \pm)}} \sim \mathcal{O}\left(\bar{r} \frac{r}{H_{\rho}}\right) \sim 4 \cdot 10^{-3} .
$$



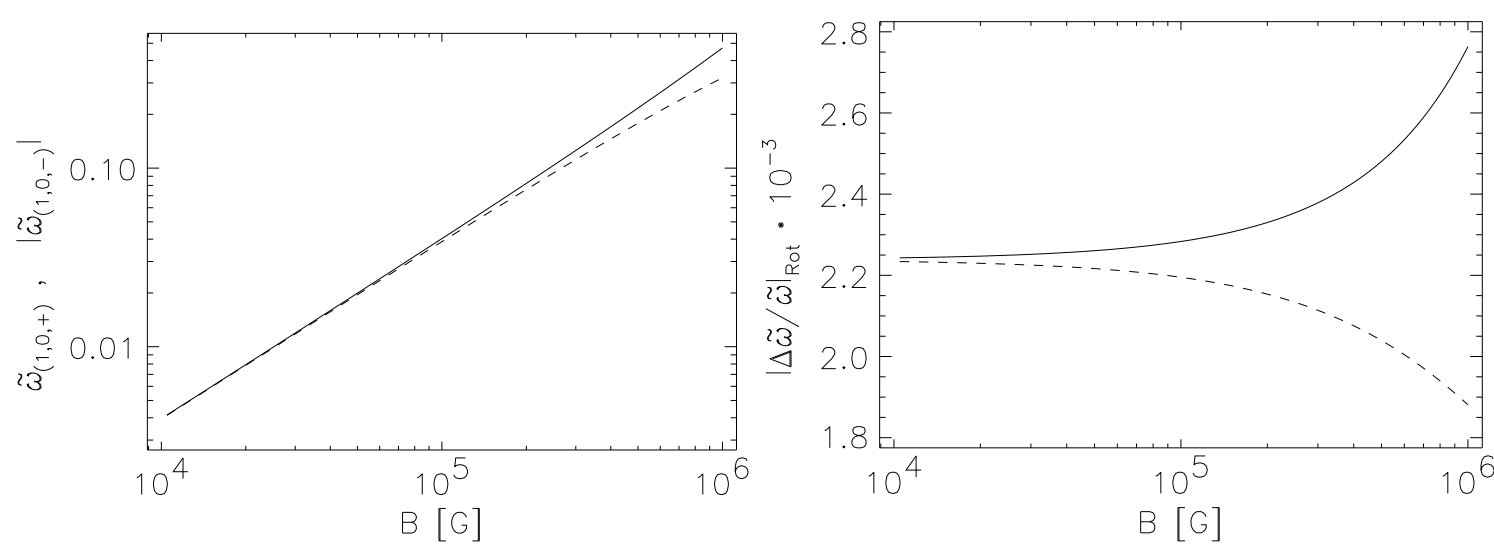

ABBILDUNG 9.4: Basisfrequenzen binormaler Eigenmodi $\xi_{\mathrm{b}}$ (links) und ihre relative Abweichung aufgrund der Rotationsabplattung (rechts) für einen Flussring in der Äquatorebene eines Einzelsterns. Prograde Wellenbewegung für $\tilde{\omega}_{(1,0,-)}$ (durchgezogene Linie) und retrograde Wellenbewegung für $\tilde{\omega}_{(1,0,+)}$ (gestrichelte Linie).

Diese Abschätzung ist nur unter der Bedingung $M_{\alpha 0} \ll 1$ zulässig, welche für die hier relevanten Gleichgewichte sehr gut erfüllt ist (Referenzfall: $B \simeq 10^{5} \mathrm{G} \Longrightarrow M_{\alpha 0} \sim 10^{-2}$ ). Die rotationsbedingte Änderung der Umgebung des Flussrings entspricht einer sehr kleinen radialen Verschiebung seiner ursprünglichen Gleichgewichtslage. Da aber die Ausgangslage einer Flussröhre im Rahmen des Modells beliebig gewählt werden kann, ist dieser Effekt hier im Grunde uninteressant. Bei der folgenden analytischen Behandlung werden diese Frequenzverschiebungen deswegen ignoriert und die Eigenfrequenzen

$$
\tilde{\omega}_{(m, 0, \pm)}=m \frac{2 M_{\alpha 0}}{M_{\alpha 0} \pm 1}
$$

verwendet. Deren Verlauf ist in Abb. 9.4 (links) als Funktion der Magnetfeldstärke dargestellt, während auf der rechten Seite zum Vergleich die durch die Abplattung bewirkte relative Frequenzverschiebung (9.27) angegeben ist.

\subsubsection{Doppelsternproblem}

Im Doppelsternproblem muss in der Gl. (9.25) wegen $\tilde{c} \neq 0$ die Kopplung an die benachbarten Wellenmodi $\hat{\xi}_{\mathrm{b}, \pm 2}$ berücksichtigt und das vollständige Gleichungssystem (9.16) gelöst werden. Dazu bietet sich die Methode der Reihenbruchentwicklung an (Morse \& Feshbach 1953, Abramowitz \& Stegun 1972), da dieser Formalismus aufgrund der geringen Kopplung bereits nach dem ersten Entwicklungsschritt abgebrochen werden kann und man dadurch eine relativ einfache analytische Näherung erhält.

Um die Eigenmodi des Doppelsternproblems $\mathrm{zu}$ bestimmen, wird von den Einzelstern-Frequenzen (9.28) ausgegangen. Für typische Werte von $\tilde{\omega}_{(1,0, \pm)}$ (Abb. 9.4, links) und kleine Wellenzahlen $m$ werden die benötigten Amplitudenverhältnisse durch die Ausdrücke

$$
\frac{\hat{\xi}_{\mathrm{b}, 2}}{\hat{\xi}_{\mathrm{b}, 0}} \simeq-\frac{\tilde{c} m^{2} \tilde{\omega}_{(1,0, \pm)}^{2}}{8+4 m\left(2-\tilde{\omega}_{(1,0, \pm)}\right)}
$$




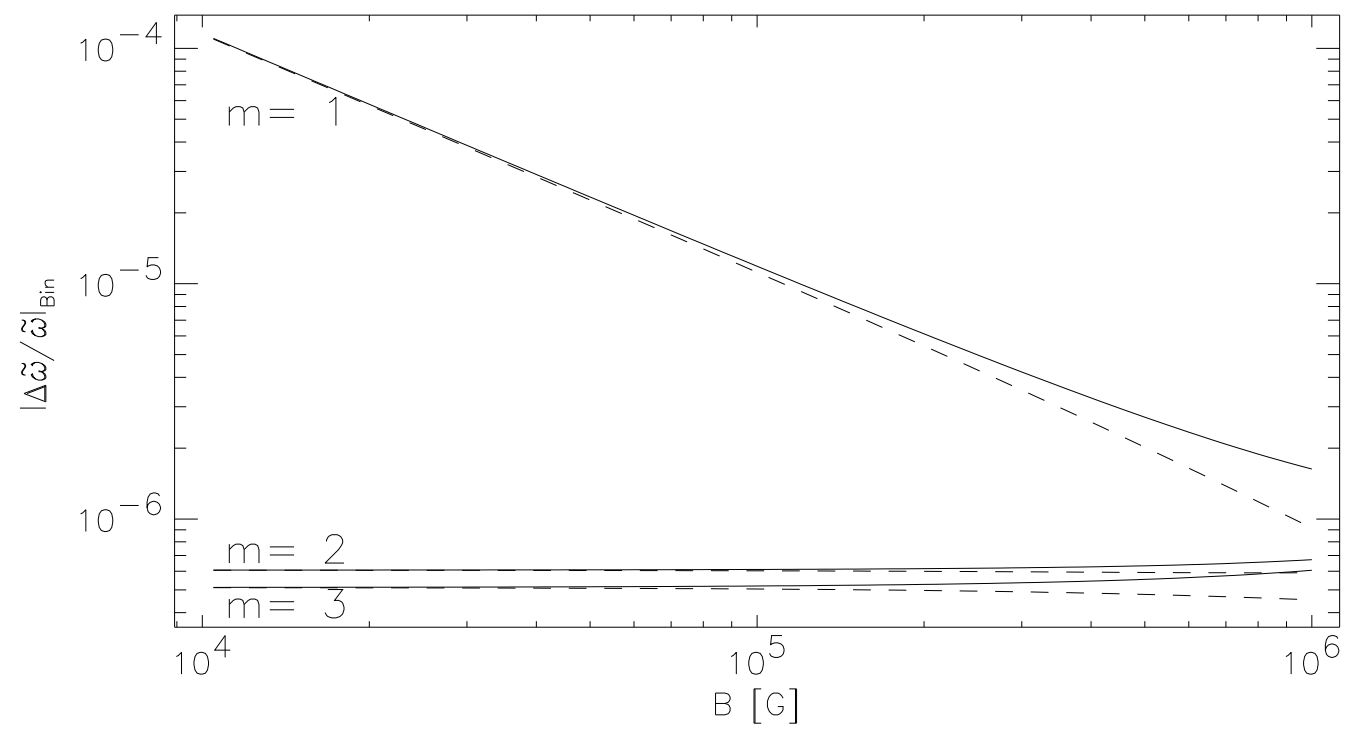

ABBILDUNG 9.5: Relative Frequenzabweichung aufgrund des Doppelsterncharakters der prograden (durchgezogene Linie) und retrograden Wellenbewegung (gestrichelte Linie) der Eigenmodi $m=1 \ldots 3$

und

$$
\frac{\hat{\xi}_{\mathrm{b},-2}}{\hat{\xi}_{\mathrm{b}, 0}} \simeq-\frac{\tilde{c} m^{2} \tilde{\omega}_{(1,0, \pm)}^{2}}{8-4 m\left(2-\tilde{\omega}_{(1,0, \pm)}\right)}
$$

approximiert. Nach der Substitution in Gl. (9.25) erhält man

$$
f_{\epsilon} \simeq \tilde{c}^{2} \tilde{\omega}_{(1,0,+)}^{2} \tilde{\omega}_{(1,0,-)}^{2} \frac{m^{2}\left(1-\tilde{\omega}_{(1,0, \pm)}\right)}{8-2 m^{2}\left(2-\tilde{\omega}_{(1,0, \pm)}\right)^{2}}
$$

und die Eigenfrequenzen $\tilde{\omega}_{(m, \tilde{r}, \pm)}$ des Doppelsternproblems ${ }^{5}$. Für die Referenz-Flussröhre gilt hier die Abschätzung

$$
\left.\frac{\Delta \tilde{\omega}}{\tilde{\omega}}\right|_{\text {Bin }}=\frac{\tilde{\omega}_{(1, \tilde{r}, \pm)}-\tilde{\omega}_{(1,0, \pm)}}{\tilde{\omega}_{(1,0, \pm)}} \sim \mathcal{O}\left[\left(\tilde{r} \frac{r}{H_{\rho}}\right)^{2}\right] \sim 2 \cdot 10^{-5} .
$$

Die relativen Abweichungen der Eigenfrequenzen von denen des Einzelsternproblems (9.28) sind für die Modi $m=1 \ldots 3$ in Abb. 9.5 dargestellt. Die Frequenzänderungen $(\propto$ $f_{\epsilon} \propto \tilde{c}^{2}$ ) sind wesentlich kleiner als die zugrundeliegende Variation des Gleichgewichts $(\propto \tilde{c})$ in Gl. (9.22) und liegen typischerweise deutlich unterhalb des Schätzwerts (9.32). Der Eigenmodus $m=1$ bildet dabei eine Ausnahme, auf deren Ursache weiter unten genauer eingegangen wird.

Durch Einsetzen der Amplitudenverhältnisse (9.29) und (9.30) in die Ausgangsgleichungen (9.18) - (9.21) erhält man Näherungen für die einhüllende Kurve $\left|\hat{\xi}_{\mathrm{b}}\right|$ der Eigenfunktion und die Phasengeschwindigkeit $v_{\mathrm{p}}$ der Welle. Beide Größen sind im Gegensatz

\footnotetext{
${ }^{5}$ Für $\tilde{\omega}_{(m, \tilde{r},+)}$ verwendet man in den Gln. (9.29) - (9.31) die Größe $\tilde{\omega}_{(1,0,+)}$ und für $\tilde{\omega}_{(m, \tilde{r},-)}$ entsprechend $\tilde{\omega}_{(1,0,-)}$.
} 
zum Einzelsternproblem Funktionen des Azimutwinkels $\phi$. Im Weiteren gelte per Definition die Normierung

$$
\hat{\xi}_{\mathrm{b}, 0}:=1 \text {. }
$$

Alle Eigenmodi sind stabil. Das bedeutet, dass die Eigenfrequenzen $\tilde{\omega}_{(m, \tilde{r}, \pm)}$ und aufgrund der Gln. (9.29) und (9.30) auch die Amplituden $\hat{\xi}_{\mathrm{b}, \pm 2}$ reelle Größen sind. Daraus folgt für die Enveloppe der Eigenfunktion

$$
\left|\hat{\xi}_{\mathrm{b}}(\phi)\right| \simeq 1+\left|c_{\mathrm{b}}\right| \cos \left(2 \phi+\arg c_{\mathrm{b}}\right)
$$

mit

$$
\left|c_{\mathrm{b}}\right|=\left(\begin{array}{c}
\tilde{c} \\
\bar{c}
\end{array}\right) \frac{m^{2}\left(1-\tilde{\omega}_{(1,0, \pm)}\right)}{4-m^{2}\left(2-\tilde{\omega}_{(1,0, \pm)}\right)^{2}}
$$

und

$$
\arg c_{\mathrm{b}}=0 \quad \text { oder } \quad \pi .
$$

In Abb. 9.6 sind die einhüllenden Kurven der Eigenfunktionen für verschiedene Wellenzahlen und Feldstärken dargestellt, aufgrund der $\pi$-Periodizität allerdings nur für den halben Flussröhrenumfang. Die höheren Wellenmodi $m=2$ und $m=3$ sind sich sowohl bei der prograden als auch bei der retrograden Welle qualitativ und quantitativ sehr ähnlich: Die Enveloppe zeigt eine Variation im Promillebereich und die Maxima liegen bei den Quadraturpunkten $Q_{1,2}$, d.h. $\phi=\pi / 2$ und $3 \pi / 2$. Die Abhängigkeit vom Magnetfeld ist gering. Eine Ausnahme bildet wieder der $m=1$-Modus, bei dem $\left|\hat{\xi}_{\mathrm{b}}\right|$ entlang der Flussröhre um einige Prozent variiert und stark von der Magnetfeldstärke $B$ abhängt. Zudem existiert zwischen den Maxima der prograden und retrograden Welle eine Phasenverschiebung: Während bei der prograden Welle die Maxima bei den Quadraturpunkten $Q_{1,2}$ liegen, sind sie bei der retrograden Wellen bei den Punkten $V_{1,2}$ entlang der Verbindungslinie $\overline{M_{*} M_{\mathrm{co}}}$. Im Gegensatz zu den Abweichungen der Eigenfrequenzen (9.32) sind die Auswirkungen des Doppelsterncharakters hier deutlich größer als die ursächliche Variation in der Ausgangsgleichung (9.22).

Mit Hilfe der berechneten Amplitude $\hat{\xi}_{\mathrm{b}}(\phi)$ erhält man mittels Gl. (9.21) den Verlauf der Phasengeschwindigkeit $v_{\mathrm{p}}$ als Funktion des Winkels $\phi$ (Abb. 9.7). Aufgrund der Abhängigkeit von $\arg \left[\hat{\xi}_{\mathrm{b}}(\phi)\right]$ spiegelt die Variation $\Delta v_{\mathrm{p}} / v_{\mathrm{p} 0}$ der Phasengeschwindigkeit die qualitativen und quantitativen Eigenschaften der Enveloppe wider: Höhere Modi zeigen ein einheitliches Verhalten, während der $m=1$-Modus deutlich davon abweicht.

\subsubsection{Diskussion}

Der Sonderfall eines toroidalen Flussrings in der Äquatorebene eines Doppelsterns zeigt einige Stabilitätseigenschaften, die auch für Flussröhren außerhalb der Äquatorebene charakteristisch sind.

Die Auswirkungen der Rotation und des Doppelsterncharakters auf die Eigenfrequenzen und -funktionen sind sehr unterschiedlich. Die axialsymmetrische Rotationsdeformation lässt die Struktur der Eigenfunktionen unverändert, wirkt sich aber relativ stark auf 


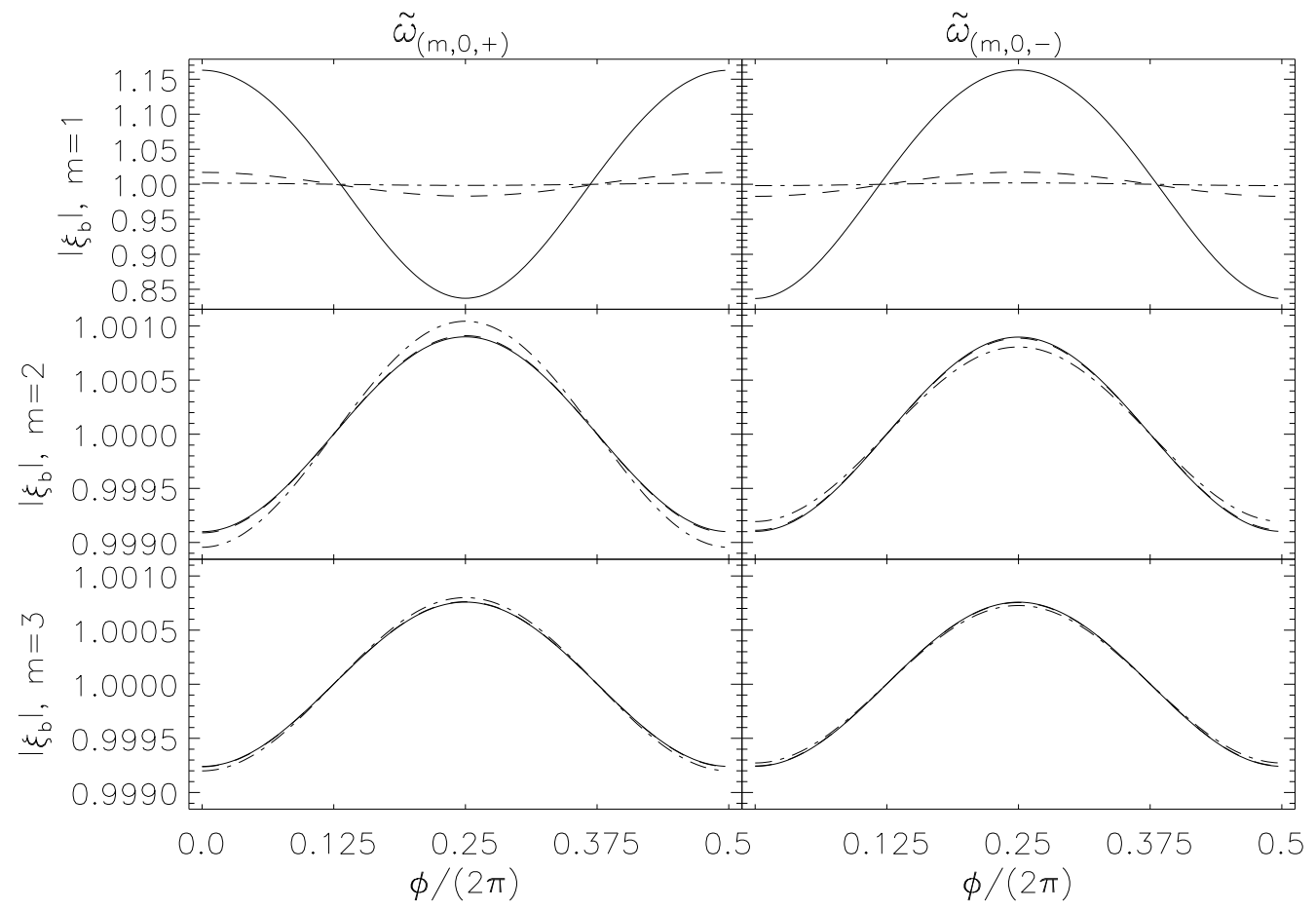

ABBILDUNG 9.6: Einhüllende Kurven $\left|\hat{\xi}_{\mathrm{b}}(\phi)\right|$ der Eigenfunktionen $m=1 \ldots 3$ für den retrograden (links) und prograden Modus (rechts). Die Kurven entsprechen unterschiedlichen Feldstärken der Referenz-Flussröhre: $B=10^{4} \mathrm{G}$ (durchgezogene Linie), $10^{5} \mathrm{G}$ (gestrichelte Linie) und $10^{6} \mathrm{G}$ (strichpunktierte Linie).

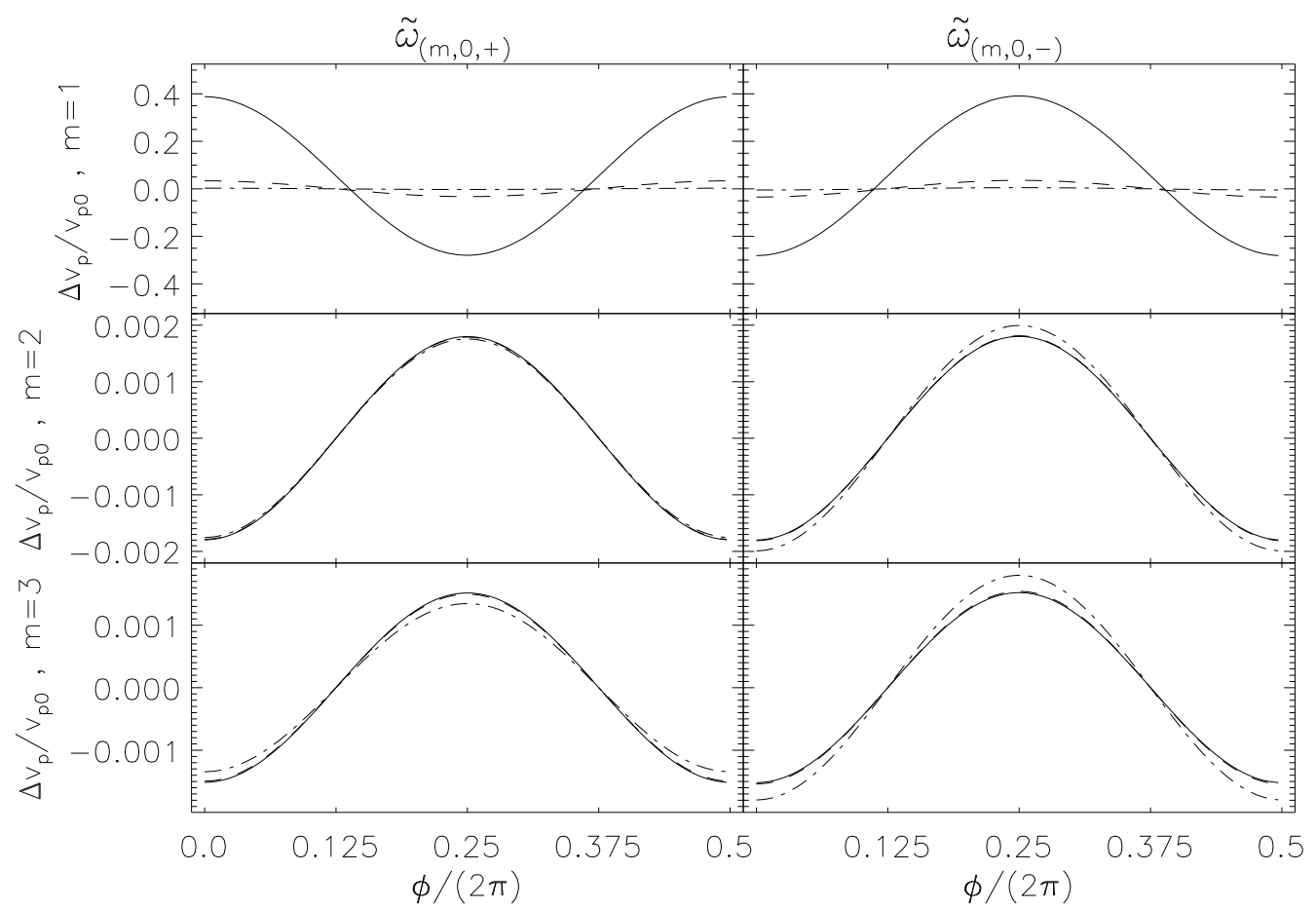

AbBILDUNG 9.7: Relative Abweichung $\Delta v_{\mathrm{p}} / v_{\mathrm{p} 0}$ der Phasengeschwindigkeit der Eigenmodi $m=1 \ldots 3$. Darstellung, Anordnung und Feldstärken wie in Abb. 9.6. 
die Eigenfrequenzen aus:

$$
\left.\frac{\Delta \tilde{\omega}}{\tilde{\omega}}\right|_{\text {Rot }} \sim 4 \cdot 10^{-3} \quad \text { und } \quad \hat{\xi}_{\mathrm{b}, k}= \begin{cases}\text { const. } & , k=0 \\ 0 & , k \neq 0\end{cases}
$$

Im Gegensatz dazu hat die Aufhebung der Rotationssymmetrie durch die Existenz des Begleitsterns einen relativ kleinen Einfluss auf die Eigenfrequenzen, aber einen sehr deutlichen auf die Struktur der Eigenfunktionen:

$$
\left.\left.\frac{\Delta \tilde{\omega}}{\tilde{\omega}}\right|_{\text {Bin }} \sim 2 \cdot 10^{-5} \ll \frac{\Delta \tilde{\omega}}{\tilde{\omega}}\right|_{\text {Rot }} \quad \text { und } \quad \hat{\xi}_{\mathrm{b}, k} \neq 0, \forall k
$$

Die Zahlenwerte gelten für den Referenzfall (Tab. 9.1 in Abschn. 9.1.1 und Tab. 8.1 in Abschn. 8.2). Die Auswirkungen des Begleitsterns auf die Struktur der Eigenfunktion sind offensichtlich: Die periodische Änderung der Enveloppe und Phasengeschwindigkeit spiegelt die Änderung der Gleichgewichtsbedingungen der jeweiligen Röhrensegmente entlang des Flussrings wider. Die sehr kleinen Frequenzverschiebungen im Doppelsternproblem erklären sich hingegen so, dass die Eigenfrequenz $\tilde{\omega}$ einen charakteristischen $\mathrm{Pa}-$ rameter der gesamten Flussröhre darstellt und in diesem Sinne einer Mittelung über alle Flussröhrensegmente entspricht. Die Wirkung der periodischen Gleichgewichtsänderung hebt sich dabei in der Summe weitgehend auf.

Gleichung (9.36) und die Kurvenverläufe in den Abbn. (9.6) und (9.7) veranschaulichen ein zusätzliches charakteristisches Stabilitätsmerkmal von Flussröhren in Doppelsternen. Die Extrema der Enveloppe $\left|\hat{\xi}_{\mathrm{b}}(\phi)\right|$ eines stabilen Eigenmodus liegen generell in der Richtung entlang der Verbindungslinie $\overline{M_{*} M_{\text {co }}}$ (bei den Punkten $V_{1,2}$, vgl. Abb. 9.1) und in der Richtung senkrecht dazu (bei den Quadraturpunkten $Q_{1,2}$ ). Die tatsächliche Lage der Maxima und Minima wird durch das Vorzeichen von $c_{\mathrm{b}}$ in Gl. (9.35) bestimmt, d.h. letztlich durch die Amplitudenverhältnisse der Partialwellen $\hat{\xi}_{\mathrm{b}, \pm 2}$. Darüber hinaus lässt diese Gleichung auf eine weitere wichtige Eigenschaft schließen. Ein instabiler Eigenmodus hat eine komplexwertige Eigenfrequenz $\tilde{\omega}$, und aufgrund der Beziehung (9.35) wird $c_{\mathrm{b}}$ ebenfalls komplexwertig sein. Wegen $\arg c_{\mathrm{b}} \neq 0$ folgt in diesem Fall eine Phasenverschiebung, wodurch sich die Lage der Extrema bezüglich der Verbindungslinie $\overline{M_{*} M_{\text {co }}}$ verschiebt und von den Punkten $V_{1,2}$ bzw. $Q_{1,2}$ wegbewegt. Die Behandlung des allgemeinen Falls im nächsten Abschnitt bestätigt diese Vermutung: Die Lage der Enveloppe-Maxima ist eine Funktion des Arguments der Eigenfrequenz $\tilde{\omega}$ und, aufgrund der Kopplung zwischen den Partialwellen, auch der Amplitudenverhältnisse $\hat{\xi}_{\mathrm{b}, k}$. Ein geschlossener, analytischer Ausdruck wie in Gl. (9.35) kann im allgemeinen Fall jedoch nicht angegeben werden.

Im letzten Abschnitt wurde bereits auf die Sonderrolle des $m=1$-Eigenmodus hingewiesen. Die im Vergleich zu den Eigenmodi mit $m \geq 2$ deutlichen Unterschiede im Verlauf der Eigenfrequenz, der Enveloppe und der Phasengeschwindigkeit ergeben sich aus der Kopplung benachbarter Partialwellen im Wellenspektrum dieses Eigenmodus. Im Einzelsternproblem haben Eigenfunktionen die Form harmonischer Wellen. Harmonische Wellen bilden aus mathematischer Sicht die Basisvektoren eines vollständigen Vektorraums, d.h. sie sind voneinander unabhängig. Zwei Wellen mit verschiedenen Wellenzahlen beeinflussen sich gegenseitig nicht, selbst wenn sie dieselbe Eigenfrequenz $\tilde{\omega}$ haben. 


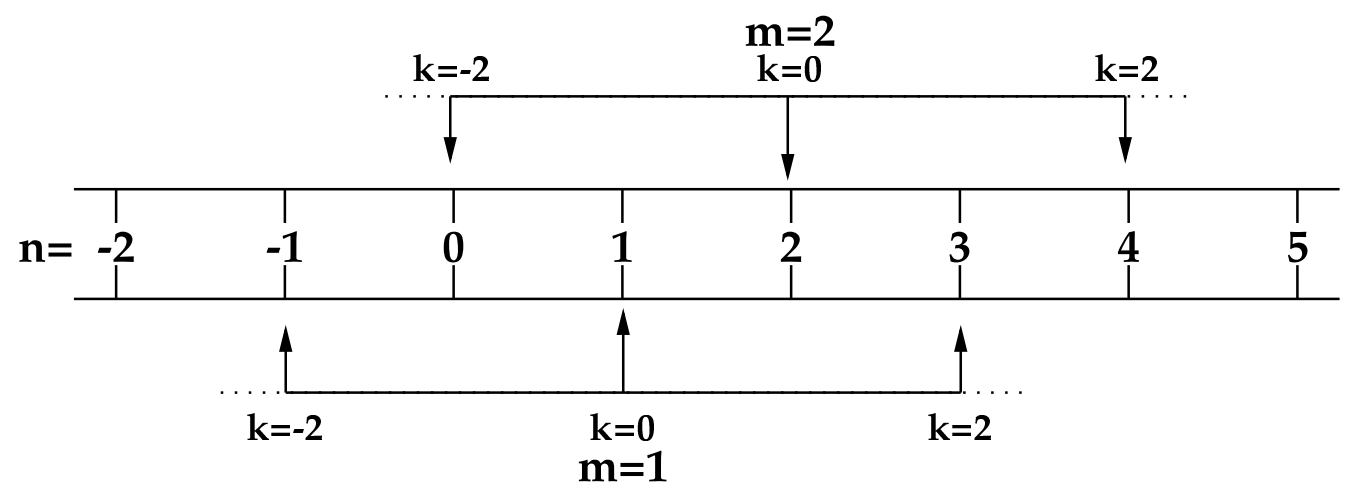

ABBILDUNG 9.8: Kopplung zwischen dem dominierenden Anteil $k=0$ eines Eigenmodus und den benachbarter Partialwellen $k= \pm 2$. Bei dem Eigenmodus $m=1$ kommt es durch die Resonanz zwischen den Partialwellen $n= \pm 1$ zu einer konstruktiven Verstärkung. Die Kopplung an weiter entfernte Partialwellen ( $|k| \geq 4$, durch Punkte angedeutet) ist in erster Näherung vernachlässigbar.

Ihre Amplituden und relativen Phasenbeziehungen werden allein durch die Anfangswerte des Problems festgelegt. Wie Gl. (9.15) zeigt, besteht im Doppelsternproblem ein Eigenmodus aus einer Überlagerung unendlich vieler Partialwellen, die zwar alle dieselbe Schwingungsfrequenz $\tilde{\omega}$, aber unterschiedliche Wellenzahlen $n=m+k, k \rightarrow \pm \infty$ haben: Der durch $m=1$ klassifizierte Eigenmodus besteht in erster Näherung aus den Partialwellen $n=m-2=-1, n=m+0=1$ und $n=m+2=3$ (vgl. Abb. 9.8). Wie man anhand Gl. (9.25) erkennen kann, ist die Eigenfrequenz $\tilde{\omega}_{(1, \tilde{r}, \pm)}$ des dominierenden Modus $n=m=1$ (betragsmäßig) gleich der Eigenfrequenz der benachbarten Partialwelle $n=-1$. Aufgrund der Kopplung wird die $n=-1$-Welle von der $n=1$-Welle angeregt und es kommt zur Resonanz: Die beiden Partialwellen überlagern sich konstruktiv und bewirken dadurch eine effektive Verstärkung, die sich in der periodischen Variation der Einhüllenden und der Phasengeschwindigkeit bemerkbar macht; deren Variation ist deutlich größer als die Variation der Gleichgewichtsgröße in der zugrundeliegenden Gl. (9.22). Wegen $\tilde{\omega}_{(m, \tilde{r}, \pm)} \propto m$ ist eine derartig effektive Verstärkung nur für $m=1$ möglich, was im Grunde einer Selbstverstärkung entspricht. Für andere Eigenmodi, z.B. $m=2$ in Abb. 9.8, kommt es zwar zu einer Anregung der benachbarten Partialwellen $n=0$ und $n=4$, aber da die Eigenfrequenzen dieser Partialwellen von der Eigenfrequenz des $n=2$-Modus sehr verschieden sind, kommt es hierbei nicht zur Resonanz. Im allgemeinen Fall sind die Eigenfrequenzen verschiedener Modi nicht mehr äquidistant und Resonanzen auch für $m \neq 1$ möglich.

Die analytische Behandlung ist nur für die entkoppelte Komponente $\xi_{\mathrm{b}}$ möglich und stellt eine Ausnahme in der Untersuchung magnetischer Flussröhren in Doppelsternen dar. Die verbleibenden Komponenten $\xi_{\mathrm{t}}$ und $\xi_{\mathrm{n}}$ bilden im Gegensatz dazu ein zweidimensionales gekoppeltes System, welches sich aus mathematischer Sicht nicht wesentlich von dem einer Flussröhre außerhalb der Äquatorebene unterscheidet. Es wird deshalb im Rahmen des allgemeinen Falls numerisch untersucht. 


\subsection{Allgemeiner Fall}

\subsubsection{Numerisches Verfahren}

Aufgrund der räumlich-periodischen Variation der Gleichgewichtsgrößen entlang eines Flussrings setzt sich eine Eigenfunktion im Doppelsternproblem aus einem Spektrum harmonischer Partialwellen zusammen, deren Amplituden $\hat{\xi}_{k}$ zusätzlich zur Eigenfrequenz $\tilde{\omega}$ bestimmt werden müssen. Die Abweichungen der Eigenfrequenzen und -funktionen von denen des Einzelsternproblems sind allerdings ausreichend klein, dass sie aus diesen sukzessive approximiert werden können. Die entdimensionierten Eigenfrequenzen $\tilde{\omega}_{m}=\omega_{m} / \Omega$ des ungestörten Einzelsternproblems ergeben sich aus der Dispersionsgleichung (2.43) unter Vernachlässigung der Rotations- und Gezeitenwirkung; der Index $m$ bezeichnet die azimutale Wellenzahl.

Im ersten Schritt wird die Veränderung der Eigenfrequenzen aufgrund der Rotation bestimmt. Die in den Koeffizienten $c_{i}$ der Gl. (2.43) auftretenden Gleichgewichtsgrößen werden durch die Näherungen (8.19) bzw. (8.15) und (8.17) ersetzt. Dabei wird die $\phi$-abhängige Gezeitenwirkung des Begleitsterns zunächst ignoriert und nur die rotationsbedingte, axialsymmetrische Änderung der Größen berücksichtigt. Die Lösungen des Polynoms 6. Grades sind die Eigenfrequenzen $\tilde{\omega}_{\text {rot }}$ einer Flussröhre in einem rotierenden Einzelstern ${ }^{6}$. Die Ergebnisse der Stabilitätsanalyse werden bei den Simulationen in Kap. 10 verwendet, die die vollständige Rotations- und Gezeitenwirkung umfassen. Deshalb wird im Gegensatz zur analytischen Behandlung in Abschn. 9.3 die rotationsbedingte Frequenzverschiebung in der weiteren Behandlung mit berücksichtigt.

Die Eigenfrequenzen des Doppelsternproblems ergeben sich aus dem Gleichungssystem (9.17). Eine vollständige Lösung der beidseitig-unendlichen Hillmatrix $\mathcal{H}$ ist nicht möglich. Da aber die durch die Nebendiagonalmatrizen $\mathcal{L}$ und $\mathcal{R}$ vermittelte Kopplung zwischen benachbarten Wellen klein ist, kann man das Wellenspektrum auf eine endliche Anzahl von Komponenten begrenzen und die Hillmatrix bei einer endlichen Zahl $K$ von Partialwellen abschneiden:

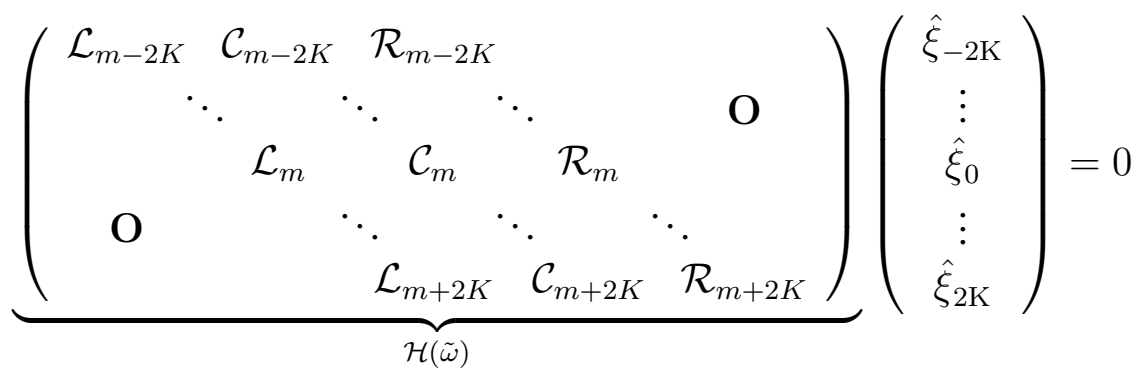

${ }^{6}$ Ein anderes Berechnungsverfahren wäre die Lösung der Gleichung

$$
\operatorname{det}\left[\mathcal{C}_{m}\left(\tilde{\omega}_{\text {rot }}\right)\right]=0 \quad, \forall m,
$$

wobei die Matrizen $\mathcal{C}_{m}$ die rotationsbedingte Änderung der Gleichgewichtsgrößen beinhalten. Diese Methode entspricht dem Lösen der Gl. (9.17), wobei nur die Blockmatrizen $\mathcal{C}_{m}$ auf der Hauptdiagonalen von Null verschieden sind, die obere und untere Nebendiagonalen, die den Gezeiteneinfluss des Begleitsterns beschreiben, jedoch verschwinden. Die Bestimmung mit Hilfe des expliziten Dispersionspolynoms hat sich jedoch als stabiler und effektiver erwiesen. 
Die explizite Form der Blockmatrizen $\mathcal{C}, \mathcal{L}$ und $\mathcal{R}$ ist in Anhang A angegeben. Die Eigenfrequenzen $\tilde{\omega}_{\text {Hill }}$ werden durch eine iterative Nullstellenbestimmung der Dispersionsgleichung

$$
\operatorname{det}\left[\mathcal{H}\left(\tilde{\omega}_{\text {Hill }}\right)\right]=0
$$

berechnet; der Startwert der Iteration ist die zuvor bestimmte Eigenfrequenz $\tilde{\omega}_{\text {rot }}$ des Einzelsternproblems mit Rotation. Testrechnungen zeigen, dass bereits bei $K=1 \ldots 2$ robuste Resultate (im Rahmen der Rechengenauigkeit) für $\tilde{\omega}_{\text {Hill }}$ erzielt werden. Für die folgenden Rechnungen wurde $K=3$ gewählt. Partialwellen, deren Wellenzahl mehr als $2 K$ von dem dominierenden Wellenmodus $m$ abweicht, haben praktisch keinen Einfluss auf das Ergebnis.

Nach dem Lösen der Gl. (9.41) können mit den bekannten Eigenfrequenzen $\tilde{\omega}_{\text {Hill }}$ die Amplituden $\hat{\xi}_{k}$ aus dem Gleichungssystem (9.40) bestimmt werden. Zur Bestimmung der genauen Eigenfunktion (und zur Kontrolle) ist es vorteilhaft, ein weiteres Verfahren zu verwenden (vgl. Collatz 1970, Kap. II, §8): Die Lösungen $x_{(i)}(\phi), i=1, \ldots, n$ eines homogenen Systems von Differentialgleichungen,

$$
x^{\prime}=\mathcal{A}(\phi) x \quad \text { mit } \quad \mathcal{A}(\phi+2 \pi)=\mathcal{A}(\phi),
$$

bilden ein Fundamentalsystem $\mathcal{X}(\phi)=\left(x_{(1)}, \ldots, x_{(\mathrm{n})}\right) \operatorname{mit} \operatorname{det}[\mathcal{X}(\phi)] \neq 0$. Mit Hilfe der Floquet-Theorie lässt sich zeigen, dass

$$
\mathcal{X}(\phi+2 \pi)=\mathcal{X}(\phi) \mathcal{M}
$$

gilt, mit der Übergangs- oder Monodromie-Matrix $\mathcal{M}=$ const. und $\operatorname{det}(\mathcal{M}) \neq 0$. Die Eigenwerte von $\mathcal{M}$ nennt man charakteristische oder Floquet-Multiplikatoren. Jede Lösung der Gl. (9.42) kann mit Hilfe eines konstanten Vektors c durch $x(\phi)=\mathcal{X}(\phi)$ c dargestellt werden. Daraus folgt

$$
x(\phi+2 \pi)=\mathcal{X}(\phi+2 \pi) \mathbf{c}=\mathcal{X}(\phi) \mathcal{M} \mathbf{c}
$$

Die geschlossene Flussröhre verlangt nach periodischen Lösungen mit $x(\phi+2 \pi) \equiv x(\phi)$. Daraus folgt

$$
\mathcal{X}(\phi) \mathcal{M c}=\mathcal{X}(\phi) \mathbf{c} \quad \text { bzw. } \quad \mathcal{M} \mathbf{c}=\mathbf{c} .
$$

Für $2 \pi$-periodische Lösungen ist es also erforderlich, dass ein Floquet-Multiplikator gleich eins ist. Erfüllt die Fundamentalmatrix die Anfangsbedingung $\mathcal{X}(0)=\mathcal{E}$, wobei $\mathcal{E}$ die Einheitsmatrix ist, so erhält man die Übergangsmatrix durch Integration entlang eines kompletten Flussröhrenumfangs:

$$
\mathcal{X}(0)=\mathcal{E} \quad \longrightarrow \quad \mathcal{X}(2 \pi)=\mathcal{M}
$$

Die Übergangsmatrix $\mathcal{M}$ und deren Eigenwerte sind Funktionen von $\tilde{\omega}$. Führt man die Berechnungen für $\tilde{\omega}=\tilde{\omega}_{\text {Hill }}$ aus Gl. (9.41) durch, so muss sich aufgrund der Randbedingungen der Eigenwert eins ergeben. Wenn dies nicht der Fall ist, wird die Lösung iteriert, bis die Periodizität gewährleistet ist. Mit dieser Methode erhält man aufgrund der notwendigen Integration und Eigenwertbestimmung automatisch den Lösungvektor $\mathbf{c}$ und die Eigenfunktion $\xi(\phi)$. 


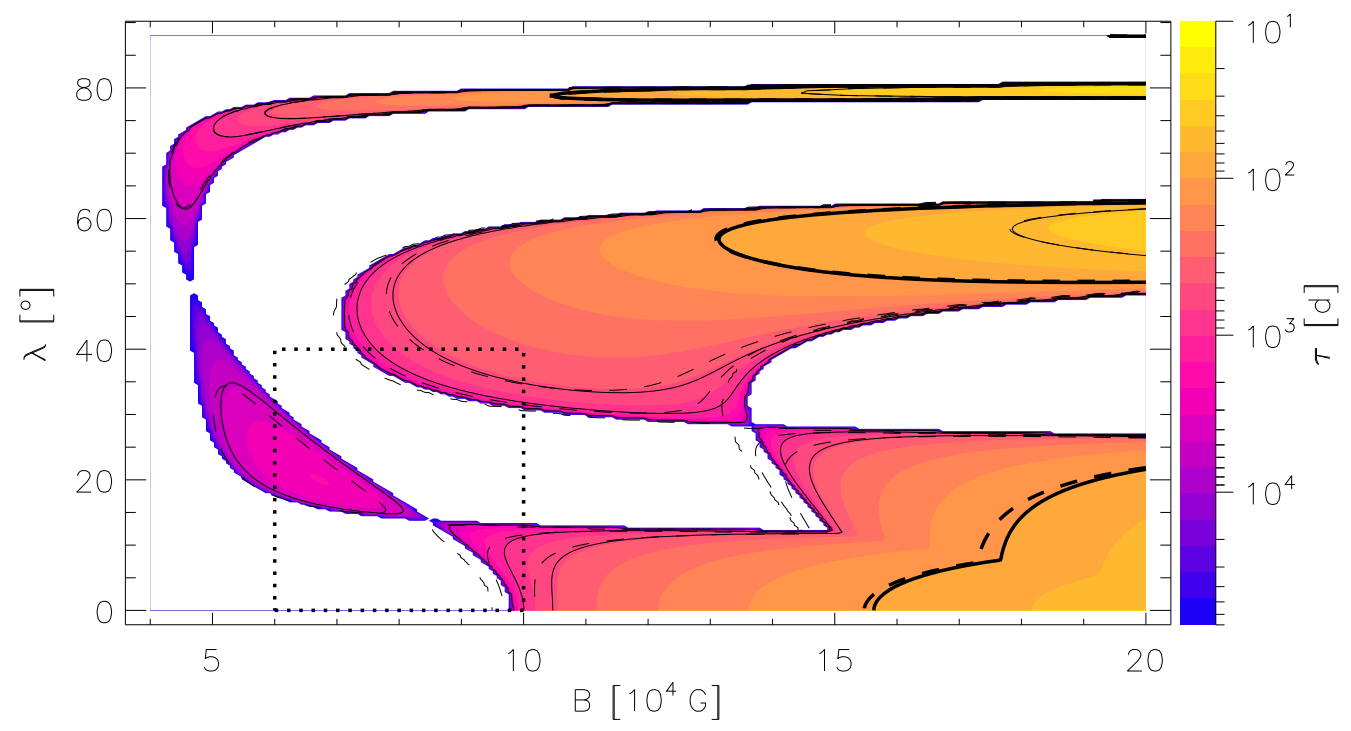

ABBILDUNG 9.9: Anwachszeiten $\tau_{\text {rot }}$ instabiler Flussröhren im Einzelsternproblem unter Berücksichtigung der Rotationsabplattung. Linien markieren Anwachszeiten $\tau_{\text {rot }}=$ $50,100,500,1000$ und 5000 d. Es gilt die Farbkodierung am rechten Rand, weiß bedeutet stabiles Gleichgewicht. Gestrichelte Linien markieren die gleichen Niveaukurven in einem Einzelstern ohne Berücksichtigung der Rotationseffekte.

\subsubsection{Ergebnisse im Referenzfall}

Im Sonderfall (9.22) in Abschn. 9.3 ist die Dichte die einzige Gleichgewichtsgröße, die entlang des Flussrings variiert, während im allgemeinen Fall alle Größen zur Winkelabhängigkeit des Gleichgewichts beitragen. Betrachtet man nur auftriebsbedingte Instabilitäten, die maßgeblich von der Superadiabatizität $\delta$ abhängen, so kann man allerdings alle anderen Gleichgewichtsvariationen ignorieren, da, wie in Abb. 9.2 auf Seite 78 gezeigt ist, die Änderung von $\delta(\phi)$ aufgrund ihrer deutlich kleineren Skalenhöhe wesentlich stärker ist als die jeder anderen $\phi$-abhängigen Größe. Bei Instabilitäten, denen ein anderer Mechanismus zugrunde liegt und die infolgedessen von weiteren Gleichgewichtsgrößen abhängen, ist dies nicht mehr zwangsläufig der Fall.

\section{Eigenfrequenzen}

EINZELSTERnPROBLEM: Das Stabilitätsdiagramm in Abb. 9.9 zeigt die Anwachszeiten $\tau_{\text {rot }}$ instabiler Flussröhren in einem Einzelstern unter Berücksichtigung der Rotationsabplattung aufgrund der Zentrifugalbeschleunigung. Zum Vergleich sind zusätzlich die selben Niveaukurven des ungestörten Einzelsternproblems, d.h. ohne Rotations- oder Gezeiteneffekte, als gestrichelte Linien eingezeichnet. Wegen der Abplattung des Sternkörpers liegt ein Flussring (bei unverändertem $r$ und $\lambda$ ) etwas tiefer in der Overshoot-Region. Da die Superadiabatizität $\delta$ dort kleiner (d.h. die Sternschichtung etwas stabiler) ist, werden höhere Feldstärken benötigt, um das ursprüngliche Niveau der Anwachsrate (ohne Rotationseffekte) zu erreichen; die maximale Änderung des Magnetfelds beträgt im vorliegenden Referenzfall lediglich $\Delta B \lesssim 5 \cdot 10^{3} \mathrm{G}$. Die von den Polen zum Äquator mit zuneh- 
mendem Achsenabstand anwachsende Abplattung spiegelt sich in der Verschiebung der $\tau$-Niveaus wider: Bei niedrigen Breiten ist sie am größten, von mittleren zu hohen Breiten dagegen immer weniger von Bedeutung. Die Lage der Flussröhre in der OvershootRegion wird durch das Modell nicht explizit vorgegeben und kann im Prinzip frei gewählt werden. Die Änderung der Eigenfrequenzen aufgrund der Rotationsabplattung ist daher nur von untergeordneter Bedeutung.

DOPPELSTERNPROBLEM: Der Doppelsterncharakter führt zur Brechung der Rotationssymmetrie und infolgedessen zu grundlegenden Modifikationen des Stabilitätsverhaltens. Die quantitativen Änderungen der Anwachszeiten $\tau_{\text {bin }}$ sind allerdings im Vergleich zu einem Einzelstern mit Rotationsabplattung nur gering; in den ursprünglichen Instabilitätszonen liegt die relative Abweichung deutlich unter 1\%. Abbildung 9.10 zeigt die Änderung der Anwachszeit relativ zu einem rotierenden Einzelstern; dieser Vergleich ist nur in den ursprünglich instabilen Regionen mit $\tau_{\text {rot }} \neq 0$ möglich. Deutliche qualitative Unterschiede machen sich dagegen in den ursprünglich stabilen Parameterbereichen bemerkbar, in denen nun zusätzliche Instabilitäten auftreten. Abbildung 9.11 zeigt einen Teil des Stabilitätsdiagramms, der Details dieser neuen Instabilitäten umfasst. Instabilitäten mit sehr hohen Anwachszeiten bilden einen 'Instabilitätshintergrund', der den gesamten, ursprünglich stabilen Parameterbereich überdeckt (vgl. punktierter Rahmen in Abb. 9.9). Er besteht aus Plateaus, die von unterschiedlich starken, aber i.Allg. eng begrenzten $R e-$ sonanzen durchzogen sind.

Die Anwachszeiten in den Plateaus liegen typischerweise bei etlichen tausend Tagen, teilweise gehen sie sogar bis zu $\tau \sim 10^{7} \mathrm{~d}$. Vom Charakter handelt es sich dabei überwiegend um tangentiale Instabilitäten mit $m=0$ und $\Re(\omega)=0$, d.h. eine zeitlich monoton anwachsende Drehung des Flussrings aus seiner Gleichgewichtslage heraus, modifiziert durch kleine periodische Auslenkungen in den normalen Richtung ${ }^{7}$. Berücksichtigt man bei der Untersuchung aufgrund ihrer Dominanz nur die Variation der Superadiabatizität $\delta$ und vernachlässigt die aller anderen Gleichgewichtsgrößen (vgl. Abschn. 9.1), so verschwinden die Plateaus und zurück bleiben nur die Resonanz- und Parkerinstabilitäten. Hier allerdings, unter Berücksichtigung der Variation aller Gleichgewichtsgrößen, variiert auch die Dichte und die Tangentialkomponente der Gezeitenkraft periodisch, wodurch sich bei einer leichten Drehung des Flussrings das Einsetzen der Tangentialinstabilität erklärt; sie erzeugt den überwiegenden Teil des Hintergrunds und ist letztlich ein Resultat der in Abschn. 9.1 beschriebenen Entropievariation des Flussrings. Die Größenordnung der berechneten $\tilde{\omega}$ ist im Hintergrund z.T. vergleichbar mit der von Termen, die bei den Näherungen (8.19) bzw. (8.15) - (8.17) vernachlässigt wurden. Die entsprechenden numerischen Werte sind deshalb sehr unsicher. Da derart hohe Anwachszeiten für die Flussröhrendynamik keine Rolle spielen, werden Instabilitäten mit $\tau>\tau_{\max }=10^{4} \mathrm{~d}$ $(\approx 27 \mathrm{a} !)$ im Folgenden nicht weiter betrachtet.

Dies gilt jedoch nicht für die scharf begrenzten Resonanzen, die die Plateaus durchziehen und dabei deutlich übertreffen; ihre Anwachszeiten von $10^{3} \ldots 10^{4} \mathrm{~d}$ sind allerdings im Vergleich zur Rotationsperiode immer noch sehr lang. Sie illustrieren sehr deutlich die Auswirkungen des Doppelsterncharakters auf die Stabilitätseigenschaften, da sie ihre Existenz der Kopplung benachbarter Partialwellen im Wellenspektrum eines Eigenmo-

\footnotetext{
${ }^{7}$ Im axialsymmetrischen Einzelsternproblem ist ein tangentialer Versatz des Flussrings indifferent, da die resultierende Konfiguration zur ursprünglichen Gleichgewichtslage äquivalent ist.
} 


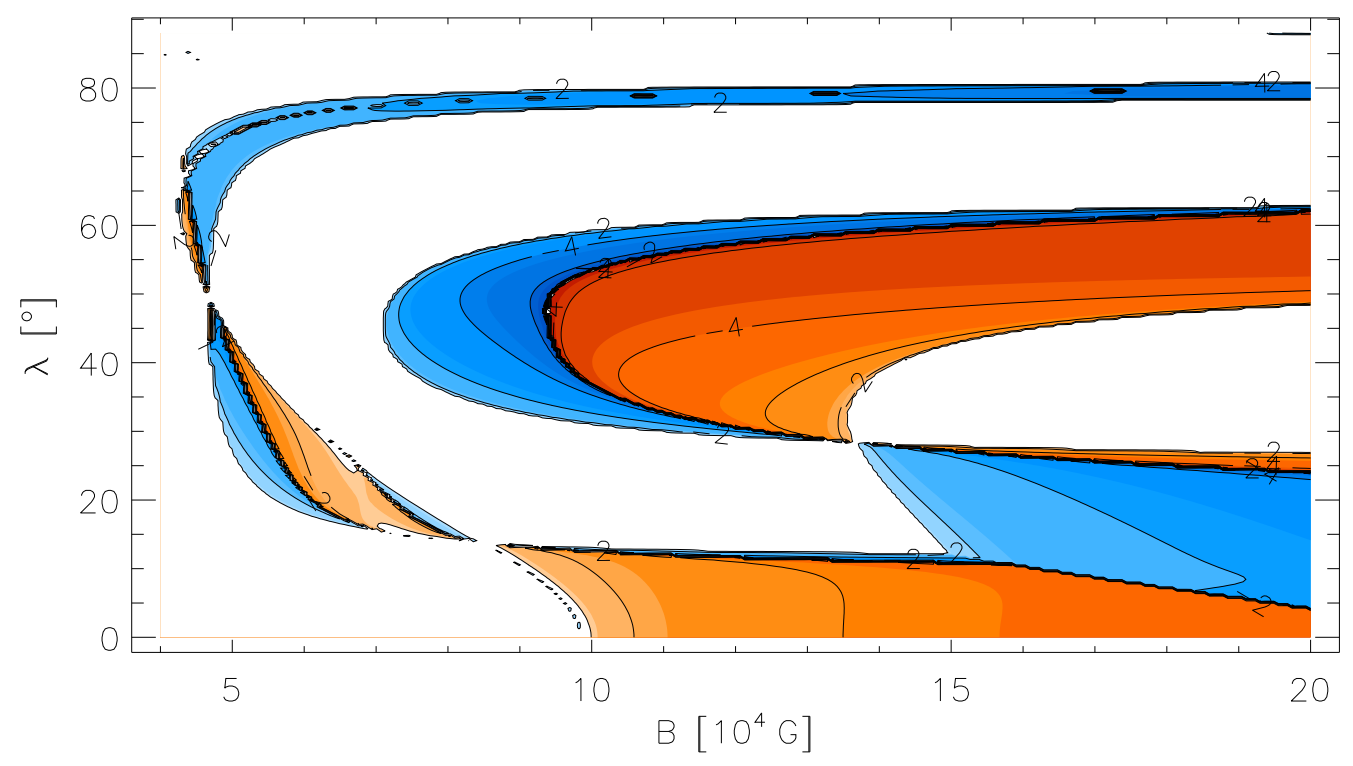

AbBILDUNG 9.10: Relative Änderung der Anwachszeiten $\left(\tau_{\text {bin }}-\tau_{\text {rot }}\right) / \tau_{\text {rot }}$ aufgrund des Doppelsterncharakters in den ursprünglich instabilen Parameterbereichen eines Einzelsterns mit Rotationsabplattung. Die Niveaulinien markieren $\log _{10}\left(|\Delta \tau| / \tau_{\text {rot }}\right)$; in den roten Gebieten ist $\tau_{\text {bin }}>\tau_{\text {rot }}$, in den blauen $\tau_{\text {bin }}<\tau_{\text {rot }}$.

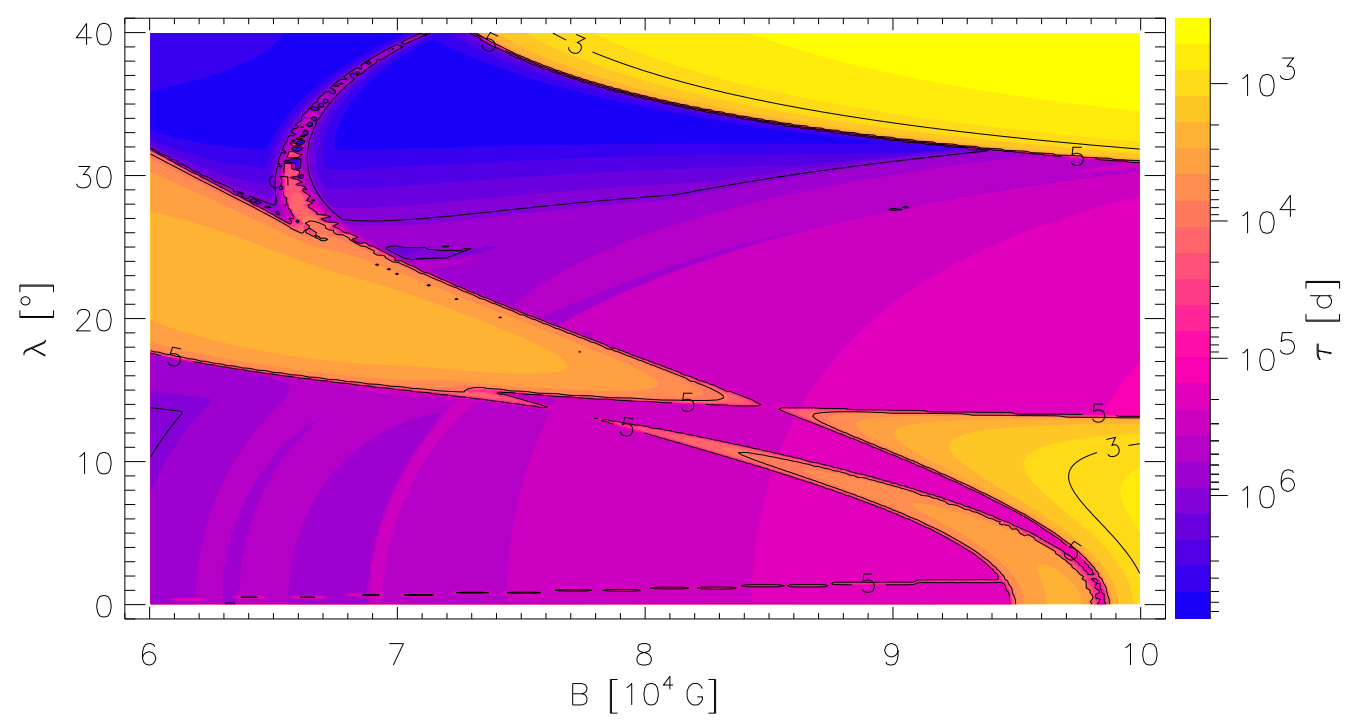

ABBILDUNG 9.11: Anwachszeiten des Instabilitätshintergrunds aufgrund des Doppelsterncharakters. Zusätzlich zur Parker-Instabilität treten nun Instabilitätsplateaus mit sehr langen Anwachszeiten auf, die von markanten Resonanzinstabilitäten durchzogen werden. Bei $\lambda \simeq 1 \ldots 2^{\circ}$ findet man eine extrem schmale, kontinuierliche Instabilität, die aus numerischen Gründen (beschränkte Auflösung) als unterbrochene Linienfolge erscheint. 


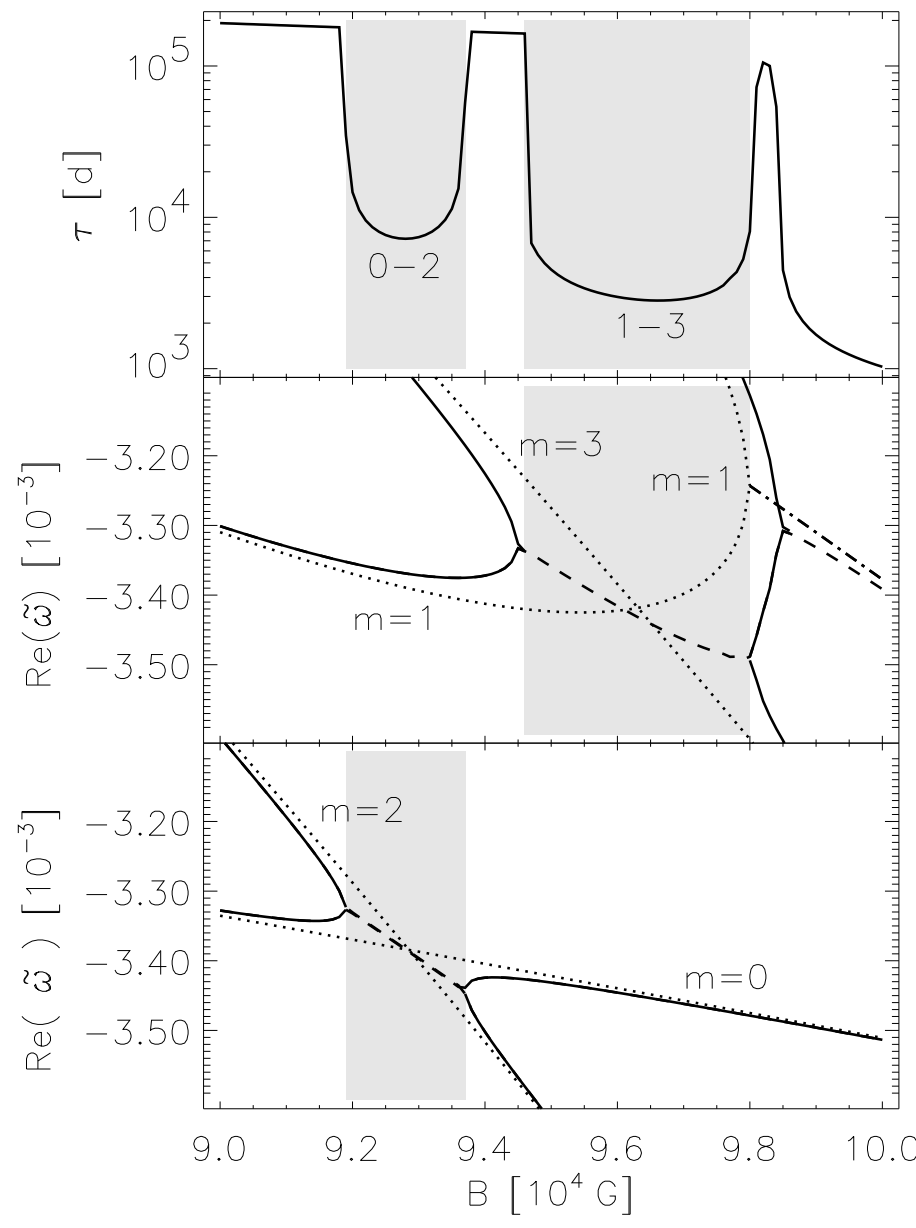

ABBILDUNG 9.12: Instabilitäten bei $\lambda=1.64^{\circ} \mathrm{im}$ Doppelsternproblem. Die Anwachszeiten der Resonanzinstabilitäten (oben, grau unterlegt) sind größer als die der ParkerInstabilität am rechten Rand. Sie entstehen durch die resonante Wechselwirkung benachbarter Partialwellen $m=1$ und 3 (Mitte) bzw. $m=0$ und 2 (unten), wenn ihre Frequenzen $\Re(\tilde{\omega})$ näherungsweise gleich sind. Die punktierten Kurven zeigen die Verläufe der nicht gekoppelten Schwingungen im Einzelsternproblem, gestrichelte bzw. strichpunktierte Linien stehen für instabile Eigenmodi mit $\Im(\tilde{\omega}) \neq 0$.

dus verdanken. Dies wird in Abb. 9.12 deutlich, die einen Schnitt durch das Diagramm 9.11 bei $\lambda=1.65^{\circ}$ zeigt. Das obere Diagramm gibt die Anwachszeiten $\tau$ wieder. Die durch Kopplung neu entstandenen Instabilitäten sind grau unterlegt, am rechten Rand setzt die äquatornahe Parker-Instabilität ein. In den Diagrammen darunter ist der Realteil $\Re(\tilde{\omega})$ der Eigenfrequenzen, d.h. die Schwingungsfrequenz verschiedener Eigenmodi aufgetragen. Die punktierten bzw. strichpunktierten Kurven gelten für das Einzelsternproblem, in dem jeder Wellenmodus $m$ selbst ein Eigenmodus ist und von den benachbarten Wellenmodi $m \pm 2$ unbeeinflusst bleibt. Die durchgezogenen bzw. gestrichelten Kurven hingegen beschreiben den Frequenzgang der Eigenmodi im Doppelsternproblem, die sich jeweils aus einem Spektrum von Partialwellen $m, m \pm 2, m \pm 4, \ldots$ zusammensetzen ${ }^{8}$. Die gestrichelten bzw. strichpunktierten Linien markieren Feldstärkebereiche, in denen $\Im(\tilde{\omega}) \neq 0$ ist und eine Instabilität vorliegt. Während sich im Einzelsternproblem die Wellen mit $m=1$ und 3 (mittleres Diagramm) bzw. $m=0$ und 2 (unteres Diagramm) bei allen Frequenzen voneinander unbeeinflusst überlagern, entsteht im Doppelsternproblem aufgrund der Kopplung eine Instabilität, wenn deren Schwingungsfrequenzen etwa übereinstimmen: Durch eine Schwingung der Partialwelle $m$ mit der Frequenz $\Re(\tilde{\omega})$ werden auch die Partialwellen $m \pm 2$ mit der selben Frequenz zu Schwingungen angeregt (vgl. Abb. 9.8). Liegt $\Re(\tilde{\omega})$ nahe bei einer der Eigenfrequenzen der benachbarten Par-

\footnotetext{
${ }^{8}$ Zum Problem der Klassifikation siehe die Erläuterungen in Abschn. 9.2.1 auf Seite 80.
} 
tialwellen, so kommt es zur Resonanz, zur konstruktiven Überlagerung und effektiven Verstärkung der Schwingung bzw. Welle und, wie in Abb. 9.12 gezeigt, gegebenenfalls zu einer Instabilität. Die Bedingungen, die zu einer instabilen Resonanz führen, hängen von dem funktionalen Zusammenhang der Gleichgewichtsgrößen ab, der im allgemeinen Fall nicht analytisch angegeben werden kann. Ein ähnliches Phänomen zeigt sich bei dem analytischen Sonderfall in Abschn. 9.3. Mit Gl. (9.31) kann dort zwar eine analytische Relation zwischen den Gleichgewichtsgrößen, der Eigenfrequenz und dem Einfluss benachbarter Partialwellen angegeben werden, der funktionale Zusammenhang führt aber lediglich zu dem Ausnahmeverhalten des $m=1$-Modus und nicht zu dem Einsetzen von Instabilitäten. Hier, im allgemeinen Fall, versteckt sich dieser wesentlich kompliziertere Zusammenhang in der Determinante der Hillschen Matrix $\mathcal{H}$ bzw. in der Übergangsmatrix $\mathcal{M}$ (vgl. Abschn. 9.4.1).

\section{Eigenfunktionen}

EINZELSTERNPROBLEM: Zur Beschreibung der Ortsabhängigkeit einer ausgelenkten Flussröhre in einem rotierenden Einzelstern wird der Ansatz (9.15) mit $\hat{\xi}_{0}=\hat{\xi}=$ const. und $\hat{\xi}_{k}=0, \forall k \neq 0$ verwendet. Eine Eigenfunktion besteht aus einer Überlagerung harmonischer Wellen gleicher Wellenzahl $m$ in den drei Richtungen $\mathbf{t}, \mathbf{n}$ und $\mathbf{b}$,

$$
\xi(\phi, t)=\left(\begin{array}{c}
\hat{\xi}_{\mathrm{t}} \\
\hat{\xi}_{\mathrm{n}} \\
\hat{\xi}_{\mathrm{b}}
\end{array}\right) e^{i(m \phi+\omega t)}=\left(\begin{array}{cc}
\left|\hat{\xi}_{\mathrm{t}}\right| & e^{i \alpha_{\mathrm{t}}} \\
\left|\hat{\xi}_{\mathrm{n}}\right| & \\
\left|\hat{\xi}_{\mathrm{b}}\right| & e^{i \alpha_{\mathrm{b}}}
\end{array}\right) e^{i(m \phi+\omega t)}
$$

bei denen typischerweise Phasenverschiebungen der Tangential- und Binormalenkomponente, $\alpha_{\mathrm{t}}$ bzw. $\alpha_{\mathrm{b}}$, bezüglich der Hauptnormalenkomponente auftreten; die Wahl der Hauptnormalen als Bezugskomponente erfolgte dabei willkürlich und o.B.d.A. Von besonderem Interesse ist die Auslenkung in der sphärisch-radialen Richtung $\mathbf{e}_{r}$, da sich die Sternschichtung in dieser Richtung am stärksten ändert. Je größer diese Auslenkung ist, desto gravierender sind die Änderungen der Umgebungsbedingungen, die ein Flussröhrenelement erfährt, da es entweder in stabilere oder instabilere Bereiche der Overshoot-Region bzw. Konvektionszone versetzt wird. Aufgrund der Linearisierung der Störung hat nur die Richtung des normierten Eigenvektors bzw. die Größe einer Komponente im Vergleich zur Gesamtauslenkung physikalischen Gehalt. Die einhüllenden Kurven in sphärisch-radialer Richtung und entlang der Breite haben die Form:

$$
\begin{aligned}
& \left|\hat{\xi}_{r}\right|^{2}=\left|\hat{\xi} \cdot \mathbf{e}_{r}\right|^{2}=\sin ^{2} \lambda\left|\hat{\xi}_{\mathrm{b}}\right|^{2}+\cos ^{2} \lambda\left|\hat{\xi}_{\mathrm{n}}\right|^{2}-\sin 2 \lambda\left|\hat{\xi}_{\mathrm{b}}\right|\left|\hat{\xi}_{\mathrm{n}}\right| \cos \alpha_{\mathrm{b}} \\
& \left|\hat{\xi}_{\lambda}\right|^{2}=\left|\hat{\xi} \cdot \mathbf{e}_{\lambda}\right|^{2}=\cos ^{2} \lambda\left|\hat{\xi}_{\mathrm{b}}\right|^{2}+\sin ^{2} \lambda\left|\hat{\xi}_{\mathrm{n}}\right|^{2}+\sin 2 \lambda\left|\hat{\xi}_{\mathrm{b}}\right|\left|\hat{\xi}_{\mathrm{n}}\right| \cos \alpha_{\mathrm{b}}
\end{aligned}
$$

Der jeweils letzte Term in diesen beiden Gleichungen entsteht beim Übergang vom begleitenden Dreibein ins Kugelkoordinatensystem durch die Phasendifferenz zwischen den Wellen in der Hauptnormalen- und Binormalenrichtung. Abbildung 9.13 zeigt einen Vergleich der Amplitudenkomponenten $\left|\hat{\xi}_{r}\right|,\left|\hat{\xi}_{\phi}\right|$ und $\left|\hat{\xi}_{\lambda}\right|$ im Kugelkoordinatensystem. Innerhalb der Instabilitätsinseln bei kleinen Feldstärken erfolgt die Auslenkung überwiegend tangential zur Sternschichtung, weshalb radiale Verschiebungen offenbar nur 


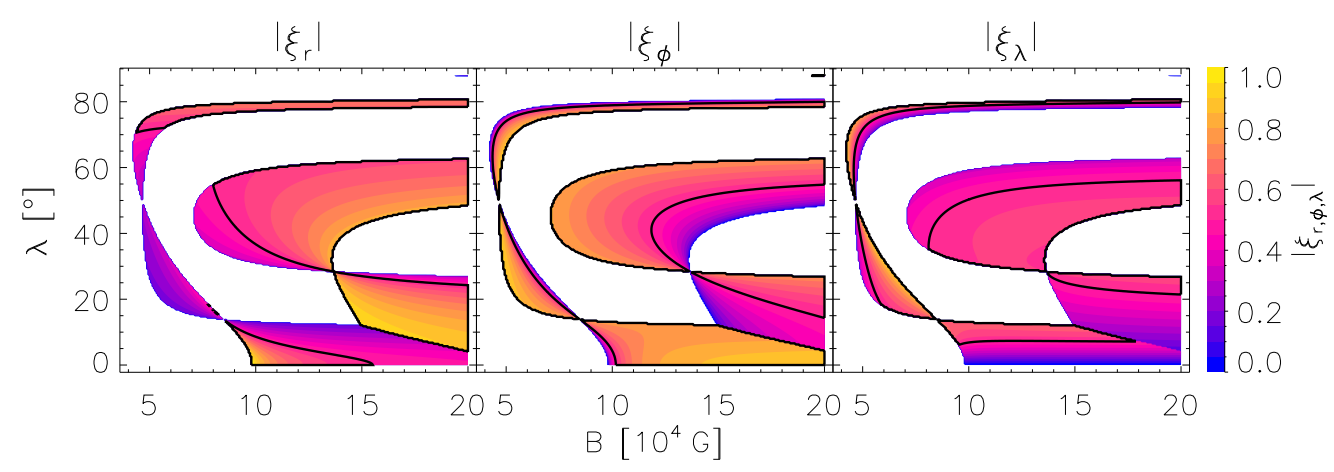

ABBILDUNG 9.13: Beträge der Eigenvektor-Komponenten im Kugelkoordinaten-System für Flussröhren in einem Einzelstern (inkl. Rotationsabplattung). Die relativen Beiträge zur Gesamtauslenkung eines Flussröhrenelements hängen in charakteristischer Weise von den beteiligten Kräften ab; es gilt die Normierung $\left|\hat{\xi}_{r}\right|^{2}+\left|\hat{\xi}_{\phi}\right|^{2}+\left|\hat{\xi}_{\lambda}\right|^{2}=|\hat{\xi}|^{2}=1$. Bereiche, in denen der jeweilige Teilbeitrag größer als $50 \%$ der Gesamtauslenkung ist, sind schwarz umrandet.

eine untergeordnete Rolle spielen. In den Bereichen der Parker-Instabilität bei höheren Feldstärken ist hingegen die radiale Komponente erwartungsgemäß groß, da dieser Typ auftriebsbedingt ist und stark von der Änderung der Umgebung (insbesondere der Superadiabatizität $\delta$ ) abhängt. Die Azimutalkomponente ist aufgrund der Drehimpulserhaltung und der daraus resultierenden Änderung der Winkelgeschwindigkeit beim Aufstieg generell von Bedeutung. Im Zusammenspiel mit der magnetischen Krümmungskraft bewirkt die Corioliskraft eine Ablenkung aufsteigender Flussröhren zu höheren Breiten, wodurch sich der Beitrag der $\left|\hat{\xi}_{\lambda}\right|$-Komponente im Bereich der Parker-Instabilität bei hohen Feldstärken erklärt. Deren hoher Anteil in den Instabilitätsinseln begründet sich dagegen eher durch den 'poleward-slip'-Mechanismus, bei dem Auftriebskräfte eine geringere Rolle spielen.

DOPPELSTERnPROBLEM: Die Auslenkung einer Flussröhre in radialer Richtung wird durch den Ausdruck

$$
\xi_{\mathrm{r}}(\phi, t)=\underbrace{\left|\hat{\xi}_{r}(\phi)\right| \exp (-\Im(\omega) t)}_{\text {Amplitude }} \underbrace{\exp \left[i\left(m \phi+\arg \hat{\xi}_{r}(\phi)+\Re(\omega) t\right)\right]}_{\text {Phase }}
$$

beschrieben. Der Amplitudenfaktor umfasst die monotone Zeitabhängigkeit und der Phasenfaktor die oszillatorische, d.h. die Wellennatur der Instabilität mit der Ausbreitungsgeschwindigkeit $v_{\mathrm{p}}$.

Mit dem Ansatz (9.15) erhält man für die Amplitude der Auslenkung den Ausdruck

$$
\hat{\xi}_{r}(\phi)=\left(\mathbf{e}_{r} \cdot \hat{\xi}(\phi)\right)=\sum_{k=-\infty}^{\infty}\left(\mathbf{e}_{r} \cdot \hat{\xi}_{k}\right) e^{i k \phi}=\sum_{k=-\infty}^{\infty}\left|\hat{\xi}_{r, k}\right| e^{i\left(k \phi+\alpha_{k}\right)} .
$$

Die Beträge der Skalarprodukte berechnen sich nach Gl. (9.48), ihre Argumente werden mit $\alpha_{k}$ bezeichnet. Die Einhüllende der Auslenkung $\left|\hat{\xi}_{r}(\phi)\right|$ ist eine periodische Funktion des Azimutwinkels $\phi$. Aufgrund der Analogie zu Gl. (9.19) können bei Dominanz 


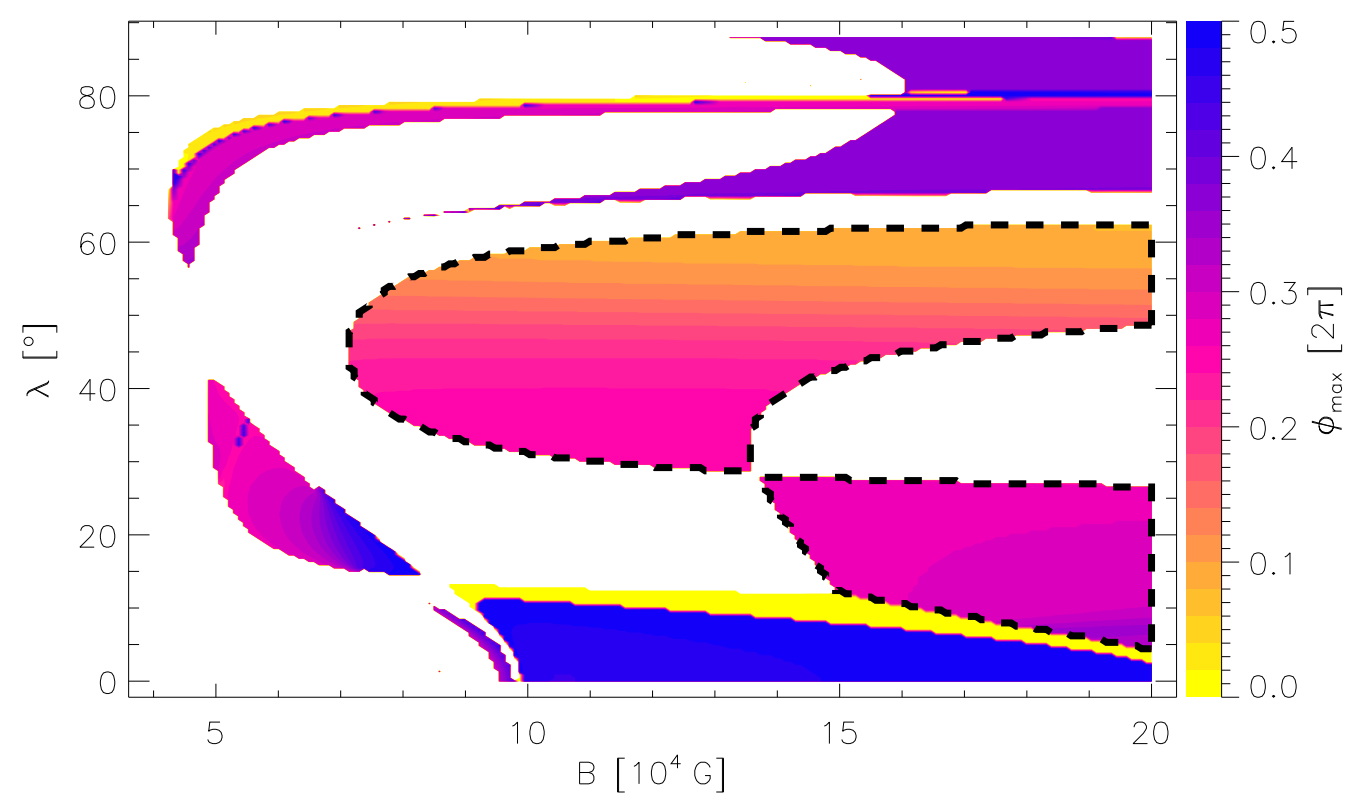

AbBILDung 9.14: Azimutwinkel $\phi_{\max }$ der Maxima der radialen Enveloppe $\left|\hat{\xi}_{r}(\phi)\right|$. Wegen der Periodizität ist nur der Wertebereich $0 \ldots \pi$ angegeben; die Werte 0 und 0.5 entsprechen den Punkten $V_{1,2}$ auf der Verbindungslinie $\overline{M_{*} M_{\mathrm{co}}}$, der Wert 0.25 dem Quadraturpunkt $Q_{1}$. Die (gestrichelt) umrahmten Gebiete sind Instabilitätsbereiche in denen die Partialwelle mit der azimutalen Wellenzahl $m=2$ dominiert.

der Partialwelle $\hat{\xi}_{r, O}$ die Winkel der Maxima mit der Beziehung (9.20) bestimmt werden. Abbildung 9.14 zeigt die Verteilung von $\phi_{\max }$ als Funktion der Breite $\lambda$ und Feldstärke $B$. Instabilitäten mit $\tau \geq \tau_{\max }=10^{4} \mathrm{~d}$ wurden dabei nicht berücksichtigt. Für $\lambda \gtrsim 60^{\circ}$ zeigt sich ein Ausläufer eines Instabilitätsplateaus mit Anwachszeiten von $\tau \gtrsim 3 \cdot 10^{3} \mathrm{~d}$. Dabei handelt es sich um eine der oben beschriebenen Tangentialinstabilitäten, die jedoch nur eine sehr kleine Komponente in radialer Richtung aufweisen. Im Gegensatz zu stabilen Eigenfunktionen, deren Maxima entweder an den Quadraturpunkten $Q_{1,2}$ oder an den Punkten $V_{1,2}$ liegen (vgl. Abschn. 9.3), zeigen die Maxima instabiler Eigenfunktionen eine Phasenverschiebung bezüglich dieser Punkte, die u.a. von der dominierenden Wellenzahl $m$ abhängt: Die Maxima der Parker-Instabilität mit $m=1$ bei niedrigen Breiten liegen meist in der Nähe der Verbindungspunkte $V_{1,2}$, während sie für $m=2$ in der weiteren Umgebung der Quadraturpunkte $Q_{1,2}$ lokalisiert sind. Bei der Auslenkung instabiler Flussröhren aus dem toroidalen Gleichgewicht werden in Abhängigkeit vom Parameterbereich und Modus demnach bestimmte Orientierungen bevorzugt.

In diesem Zusammenhang ist es wichtig, wie stark sich die Auslenkung bei verschiedenen Orientierungen unterscheidet. Als Maß dafür dient das Minimum der normierten Enveloppe mit der Bedingung $\left|\hat{\xi}_{r}\right|_{\max }=\left|\hat{\xi}_{r}\left(\phi_{\max }\right)\right|:=1$. Je kleiner das Minimum, desto größer die Unterschiede bei der Auslenkung der Flussröhre und desto größer die Wahrscheinlichkeit der Bildung einer Flussröhrenschleife in der Umgebung von $\phi_{\max }$. Abbildung 9.15 zeigt $\left|\hat{\xi}_{r}\right|_{\text {min }}$ als Funktion der Gleichgewichtsparameter $B$ und $\lambda$. Die Differenz zwischen Maxima und Minima beträgt bei höheren Feldstärken typischerweise mehrere 

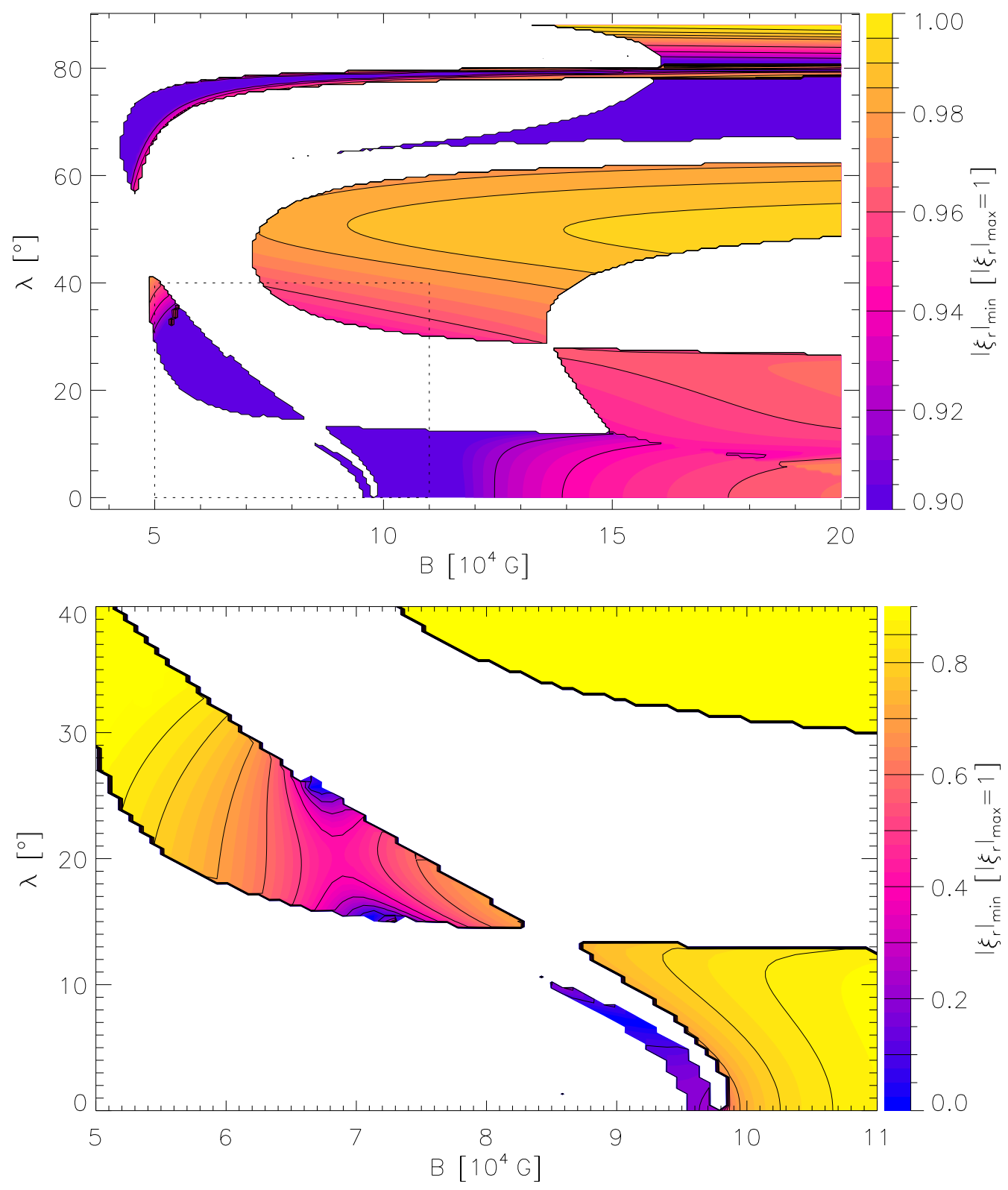

AbBILDUNG 9.15: Minima $\left|\hat{\xi}_{r}\right|_{\text {min }}$ der radialen Enveloppe im Referenzfall (Normierung: $\left|\hat{\xi}_{r}\right|_{\max }:=1$ ). In der Gesamtdarstellung (oben) wurden zusätzlich Niveaulinien eingezeichnet, die auch in der Farbkodierung am rechten Rand markiert sind. Der markierte Bereich (punktierter Rahmen) wird exemplarisch wegen seiner deutlich kleineren Werte in der unteren Abbildung separat dargestellt. Bei der Resonanzinstabilität geht $\left|\hat{\xi}_{r}\right|_{\text {min }}$ fast bis auf Null zurück. Selbst beim Durchgang durch die Instabiliätsinsel bei $\lambda \sim 20^{\circ}$ bewirkt die Resonanz eine Absenkung des Minimums $\left|\hat{\xi}_{r}\right|_{\text {min. }}$. Instabilitäten mit $\tau \geq 10^{4} \mathrm{~d}$ werden als quasi-stabil eingestuft und deswegen nicht dargestellt. 
Prozent: In mittleren Breiten $\lesssim 5 \%$ und in der Nähe des Äquators bis zu 20\%. Darüber hinaus können die Minima bei Resonanz-Instabilitäten noch erheblich kleiner werden und fast bis auf Null zurückgehen (Abb. 9.15, unten): Hier sind die Vorzugslängen sehr deutlich zu erkennen, da eine signifikante Auslenkung der Flussröhre nur noch in der Umgebung der beiden Maxima möglich, bei den Minima hingegen fast vollständig unterdrückt ist. In diesen Fällen ist die für die a priori-Näherung (9.19) notwendige Dominanz der Partialwelle $\hat{\xi}_{r, 0}$ nicht mehr gegeben. Aufgrund der Resonanz haben zwei der gekoppelten Partialwellen vergleichbare Amplituden und bilden dadurch (näherungsweise) eine stehende Welle, die zu diesem gravierenden Unterschied im Stabilitätsverhalten führt. Dieser Effekt ist vermutlich nicht von großer praktischer Bedeutung, da er nur in einem eng begrenzten Parameterbereich auftritt, in dem die Anwachszeiten mehrere tausend Tage betragen.

In Abb. 9.16 werden zum besseren Vergleich die Einhüllenden $\left|\hat{\xi}_{r}(\phi)\right|$ für instabile Eigenmodi mit $\tau \leq 1000 \mathrm{~d}$ bei verschiedenen Breiten gezeigt; zu beachten sind die wechselnden Wertebereiche der dargestellten Intervalle. Diese Kurven wurden mit der alternativen Integrationsmethode (vgl. Abschn. 9.4.1) berechnet. Für $\lambda \geq 15^{\circ}$ treten nur Eigenmodi mit $m=2$ auf, deren Enveloppe lediglich um wenige Prozent mit dem Azimutwinkel $\phi$ variiert. Die $m=1$-Modi haben ihre Maxima vergleichsweise dicht bei den Punkten $V_{1,2}$. Bei den $m=2$-Modi zeigt sich hingegen eine breitenabhängige Verschiebung der Maxima in einem weiten Bereich um die Quadraturpunkte. Typischerweise nimmt der Wert des Enveloppe-Minimums mit wachsender Feldstärke zu, d.h. die Ortsabhängigkeit der Auslenkung ist bei höheren Feldstärken geringer als bei kleinen. Wie z.B. Spruit \& van Ballegooijen (1982) oder Ferriz-Mas \& Schüssler $(1993,1995)$ gezeigt haben, geht für $\beta \gg 1$ die Abhängigkeit von der Superadiabatizität $\delta$ i.Allg. in der Form

$$
\beta \delta \propto \frac{\delta}{B^{2}}
$$

in das Stabilitätsproblem ein. Dementsprechend nimmt der Einfluss der $\delta$-Variation mit wachsender Feldstärke $B$ ab. Abbildung 9.17 gibt in einer zur Abb. 9.16 analogen Darstellung die Variation der Phasengeschwindigkeit $v_{\mathrm{p}}(\phi) / v_{\mathrm{p}, 0}$ wieder, die in Gl. (9.21) definiert wurde. Bei niederen Breiten ändert sie sich beträchtlich, bei mittleren Breiten hingegen nur um etwa 1\%. Die Phasenlage ihrer Extrema spiegelt im Wesentlichen die der Enveloppe wider.

\subsubsection{Parameterabhängigkeit}

Das Doppelsternsystem wird durch die Rotationsperiode $T$, das Massenverhältnis $q$ und die Masse des Hauptsterns $M_{*}$ charakterisiert, die durch das Keplergesetz (8.2) mit dem Sternabstand $a$ verbunden sind. Für die weitere Betrachtung wird für den Hauptstern das Modell der heutigen Sonne beibehalten. Der $\phi$-abhängige Beitrag der Gezeitenwirkung ist (für $r=$ const.) proportional zu

$$
\epsilon^{3} q \propto \frac{q}{1+q} \frac{1}{T^{2}} \quad \longrightarrow \quad \frac{\Delta\left(\epsilon^{3} q\right)}{\left(\epsilon^{3} q\right)}=\frac{1}{1+q} \frac{\Delta q}{q}-2 \frac{\Delta T}{T}
$$




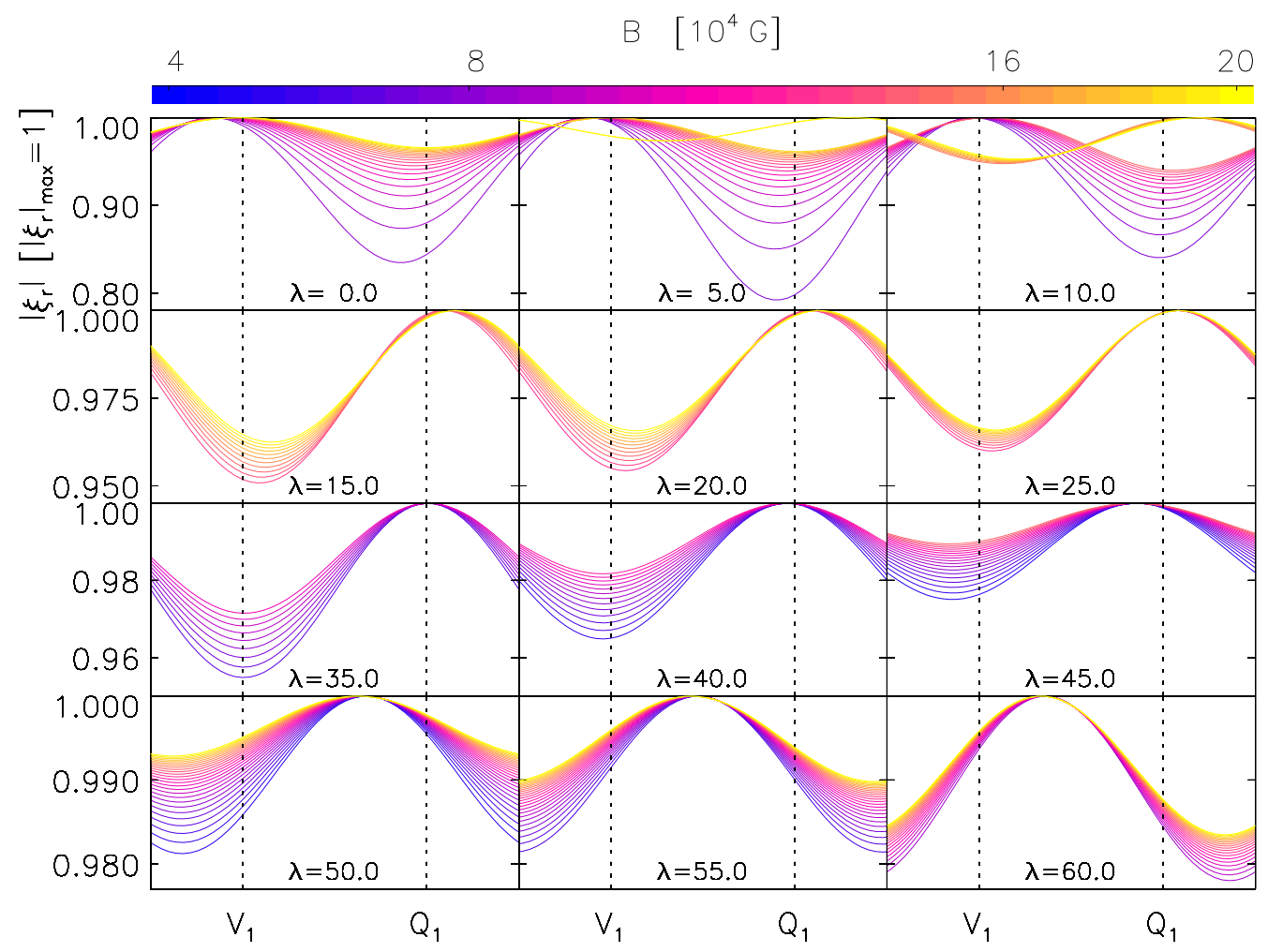

ABBILDUNG 9.16: Verlauf der radialen Enveloppe $\left|\hat{\xi}_{r}(\phi)\right|$ als Funktion der Länge $\phi$. Dargestellt sind instabile Eigenmodi mit $\tau \leq 10^{3} \mathrm{~d}$. Die Farbkodierung am oberen Rand kennzeichnet die Feldstärke $B . V_{1}$ bezeichnet den substellaren Punkt auf der Verbindungslinie, $Q_{1}$ den Quadraturpunkt. Bei $\lambda=0^{\circ}$ treten nur $m=1$-Modi auf, ab $\lambda \geq 15^{\circ}$ nur $m=2$-Modi.

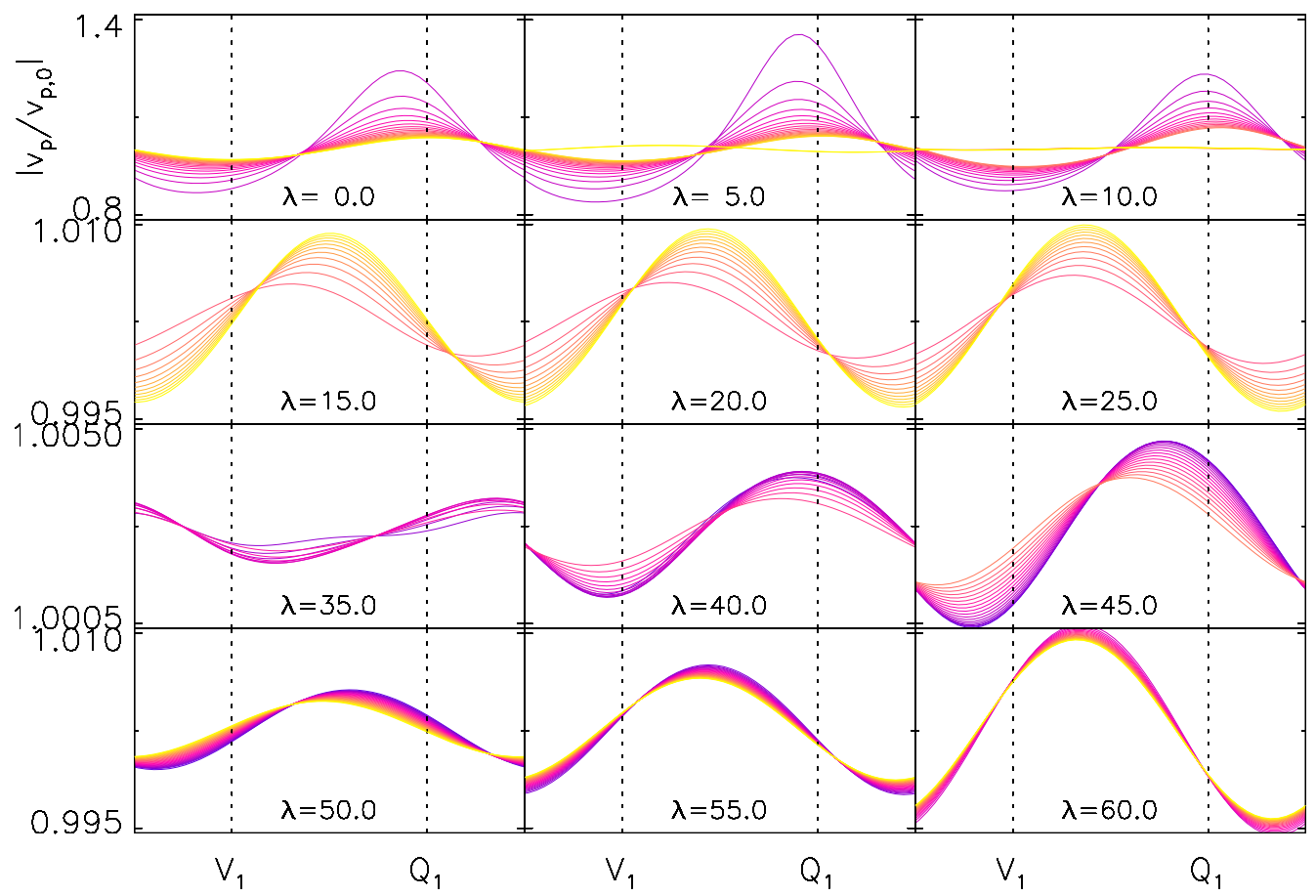

AbBILDUng 9.17: Verlauf der Phasengeschwindigkeit $v_{\mathrm{p}} / v_{\mathrm{p}, 0}$ als Funktion der Länge $\phi$. Darstellungsweise und Farbkodierung wie in Abb. 9.16. 


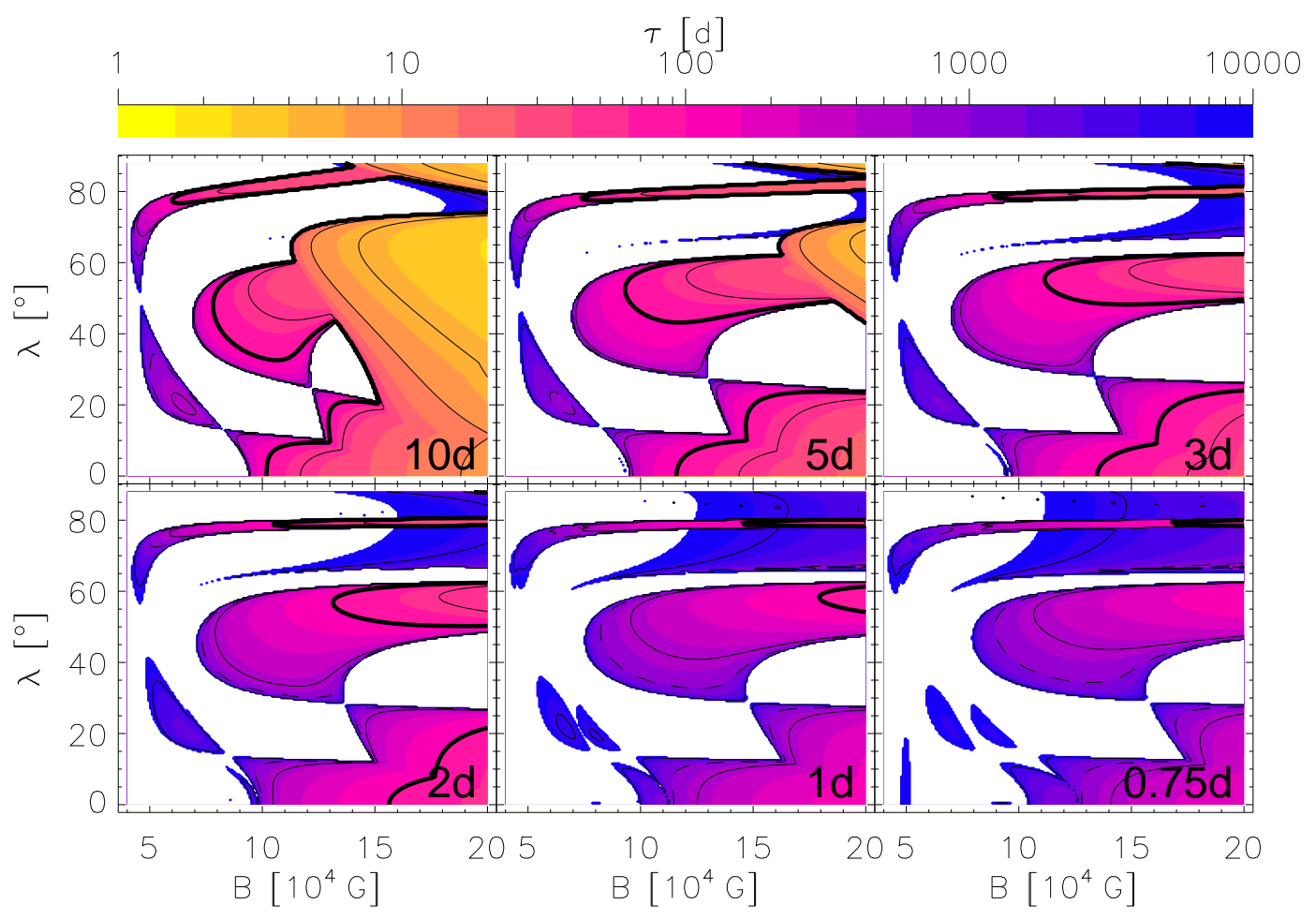

AbBILDUNG 9.18: Anwachszeiten $\tau$ instabiler Eigenmodi als Funktion der Umlaufsdauer $T$. Für alle Diagramme gilt die einheitliche Farbkodierung am oberen Rand. Die Niveaulinien entsprechen den Anwachszeiten $\tau=1,5,10,50,100,500,1000$ (gestrichelte Linie) und $5000 \mathrm{~d}$. Instabilitäten mit $\tau \geq 10^{4} \mathrm{~d}$ werden nicht dargestellt.

Ein Vergleich der Vorfaktoren zeigt, dass die relative Abhängigkeit vom Massenverhältnis vergleichsweise klein ist, sodass wir uns hier auf die Diskussion der Rotationsdauer beschränken können.

Untersucht werden enge Doppelsternsysteme mit Umlaufsperioden von $T=$ $0.75 \ldots 10 \mathrm{~d}$, bei denen der Sternabstand zwischen $a \simeq 4.4 \ldots 25 \mathrm{R}_{\odot}$ liegt (vgl. Abb. 8.2, gestrichelte Linie). Für den Entwicklungsparameter der Störungsrechnung folgt $\epsilon^{3}=$ $1.2 \cdot 10^{-2} \ldots 6.7 \cdot 10^{-5}$. Je kürzer die Rotationsdauer des Systems, desto näher ist der Begleiter am Hauptstern und desto stärker ist die Gezeitenkraft und die Änderung der Sternstruktur. Auch bei sehr schneller Rotation wird immer noch davon ausgegangen, dass die beiden Komponenten voneinander getrennt sind und der Hauptstern durch die analytische Näherungsmethode in Abschn. 8.1.2 beschrieben werden kann; deren Qualität nimmt mit fallendem $T$ jedoch kontinuierlich $a b$.

Die Stabilitätsdiagramme in Abb. 9.18 zeigen die Änderung der Anwachszeiten und die Verschiebung der Instabilitätsbereiche bei verschiedenen Rotationsperioden. Mit sinkender Rotationsperiode nimmt die Anwachszeit in den dargestellten Bereichen kontinuierlich zu; die Niveaulinie der 100 d-Instabilität verschiebt sich immer weiter zu höheren Feldstärken und ist im Extremfall $T=0.75 \mathrm{~d}$ fast verschwunden. Dieses Verhalten ist auf die stabilisierende Wirkung der Drehimpulserhaltung besonders bei kurzen Rotationsperioden zurückzuführen, die durch die Abplattung des Sterns noch verstärkt wird, 
durch welche die formale Gleichgewichtslage der Flussröhre in etwas tiefere, stabilere Schichten des Sterns verschoben wird. Im Einklang damit zeigt sich die Ausbildung einer Stabilitätsinsel bei mittleren Breiten, die sich mit wachsender Rotationsrate zu immer höheren Feldstärken erstreckt. Die tangentiale Instabilität bei hohen Breiten verstärkt sich mit kleiner werdendem $T$ und erstreckt sich letztlich bis zu kleinen Feldstärken; trotzdem bleiben die Anwachszeiten in diesem Bereich sehr hoch. Aus qualitativer Sicht ist insbesondere das Verhalten der Instabilitätsinseln bei kleinen Feldstärken zu bemerken. Durch die zunehmende Ausprägung der bei langsamerer Rotation unbedeutenden Resonanzinstabilitäten kommt es offenbar zu einer Aufteilung und einem Auseinanderlaufen der Instabilitätsinseln. Eventuell handelt es sich bei dieser Erscheinung um die Aufhebung einer Instabilitätsentartung, die im Einzelsternproblem aufgrund der Rotationssymmetrie existiert hat und im Doppelsternproblem durch die Anwesenheit des Begleiters gebrochen wird.

Die Phasenlage $\phi_{\max }$ der Maxima der Enveloppe $\left|\hat{\xi}_{r}\right|$ in radialer Richtung ist in Abb. 9.19 dargestellt. Die Werte von $\phi_{\max }$ zeigen als Funktion der Rotationsperiode keine großen Änderungen und werden im wesentlichen durch den oben beschriebenen Referenzfall $T_{\text {ref }}=2 \mathrm{~d}$ repräsentiert. Die Maxima der $m=1$-Modi liegen typischerweise nahe an der Verbindungslinie $\overline{M_{*} M_{\mathrm{co}}}$, während die der $m=2$-Modi und Instabilitätsinseln sich über ein breites Intervall von $0.1 \lesssim \phi_{\max } \lesssim 0.4$ um den Quadraturpunkt verteilen. Im Fall $T=10 \mathrm{~d}$ kann man in polnahen Breiten und hohen Feldstärken den Ansatz der $m=0$-Instabilität ('poleward slip') erkennen. Erwähnenswert ist die Verschiebung der Signatur der 0 - 2-Resonanzinstabilität bei niedrigen Breiten und höheren Feldstärken. Bei schneller Rotation liegt diese Signatur in der Nähe des Äquators und wird durch die $m=1$-Parkerinstabilität überdeckt. Mit wachsender Rotationsdauer verschiebt sie sich zu etwas höheren Breiten und bewirkt bei $T=10 \mathrm{~d}$ eine deutliche Inhomogenität der Phasenlage $\phi_{\max }$ bei $(B, \lambda) \approx\left(13 \cdot 10^{4} \mathrm{G}, 20^{\circ}\right)$. Die Anwachszeiten im Bereich dieser Erscheinung sind einige hundert Tage und weniger, sodass dieser Effekt für die Flussröhrendynamik durchaus von Bedeutung sein kann; der erwähnte Parameterbereich wird hier durch die Wahl des Referenzsystems bestimmt.

Die Minimalwerte $\left|\hat{\xi}_{r}\right|_{\text {min }}$ der radialen Enveloppe, die als Maß für die Wahrscheinlichkeit der Bildung einer Flussröhrenschleife in der Nähe von $\phi_{\max }$ dienen, sind in Abb. 9.20 dargestellt. Dabei ist der Darstellungsbereich für jedes Diagramm verschieden, um den Parameterbereich der Instabilitäten mit kleinen Anwachszeiten gut aufzulösen. Ein Vergleich der jeweiligen Darstellungsgrenzen zeigt erwartungsgemäß die eindeutige Abnahme von $\left|\hat{\xi}_{r}\right|_{\text {min }}$ mit sinkender Rotationsdauer, die den wachsenden Einfluss des Begleitsterns und der Gezeitenwirkung auf das Gleichgewicht und die Stabilitätseigenschaft widerspiegelt. Generell ist der Einfluss bei kleinen Feldstärken und insbesondere in den Instabilitätsinseln und bei den Resonanzinstabilitäten am gravierendsten: Bei kürzeren Rotationsperioden kann dort das Minimum der Enveloppe fast bis auf Null zurückgehen. Beschränkt man sich dagegen auf große Anwachsraten, so ist die Schwankung der Enveloppe wesentlich geringer: Im günstigsten Fall etwa $\sim 20 \%$, typischerweise eher im Bereich von $\sim 2-5 \%$. Dagegen sind bei einer Rotationsperioden von $T=10 \mathrm{~d}$ im Grunde keine nennenswerten Variation mehr vorhanden. 


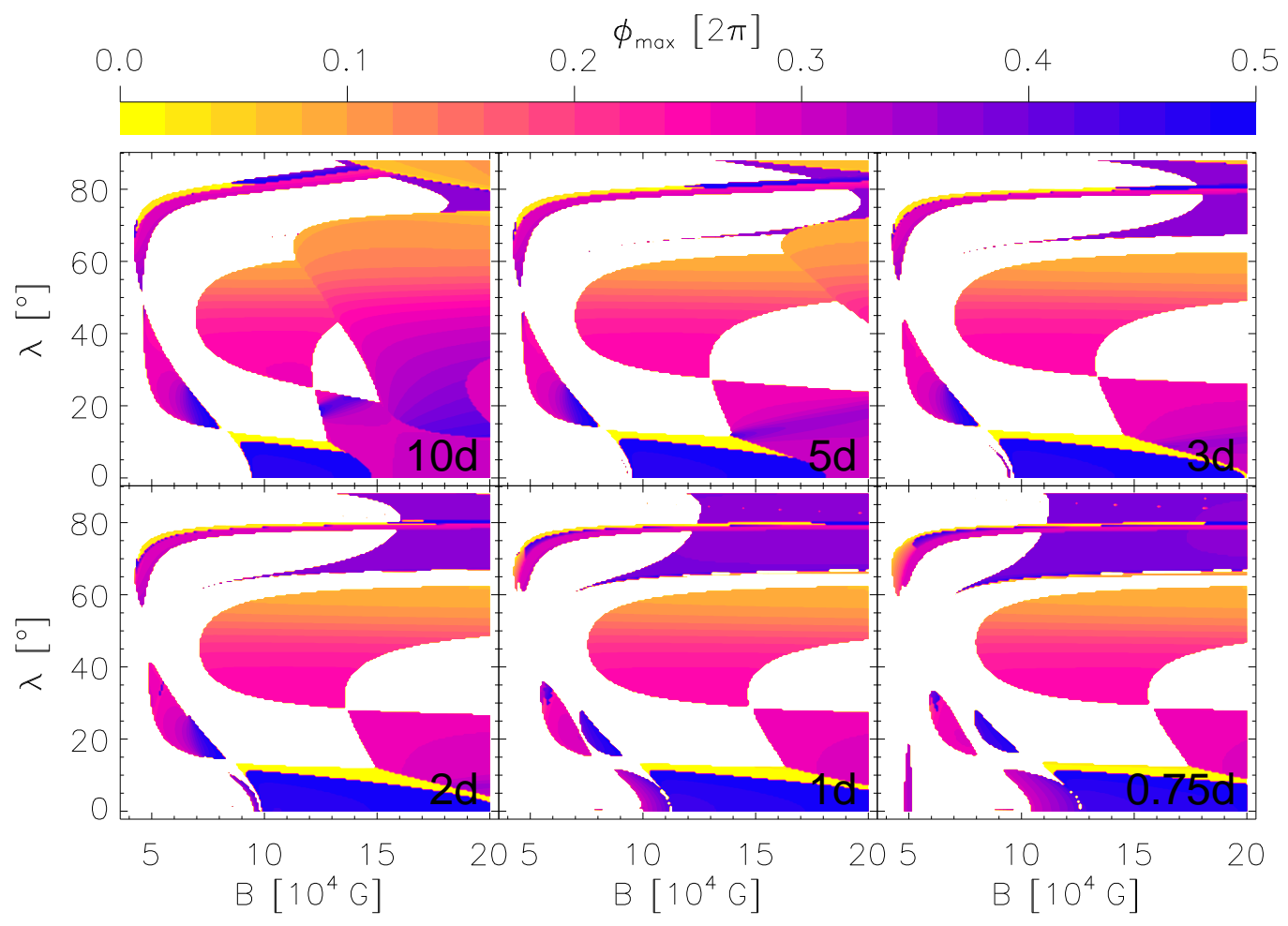

AbBILDUNG 9.19: Phasenlage $\phi_{\max }$ der Maxima der radialen Enveloppe $\left|\hat{\xi}_{r}\right|$ als Funktion der Umlaufsdauer $T$. Instabilitäten mit $\tau \geq 10^{4} \mathrm{~d}$ werden nicht dargestellt.

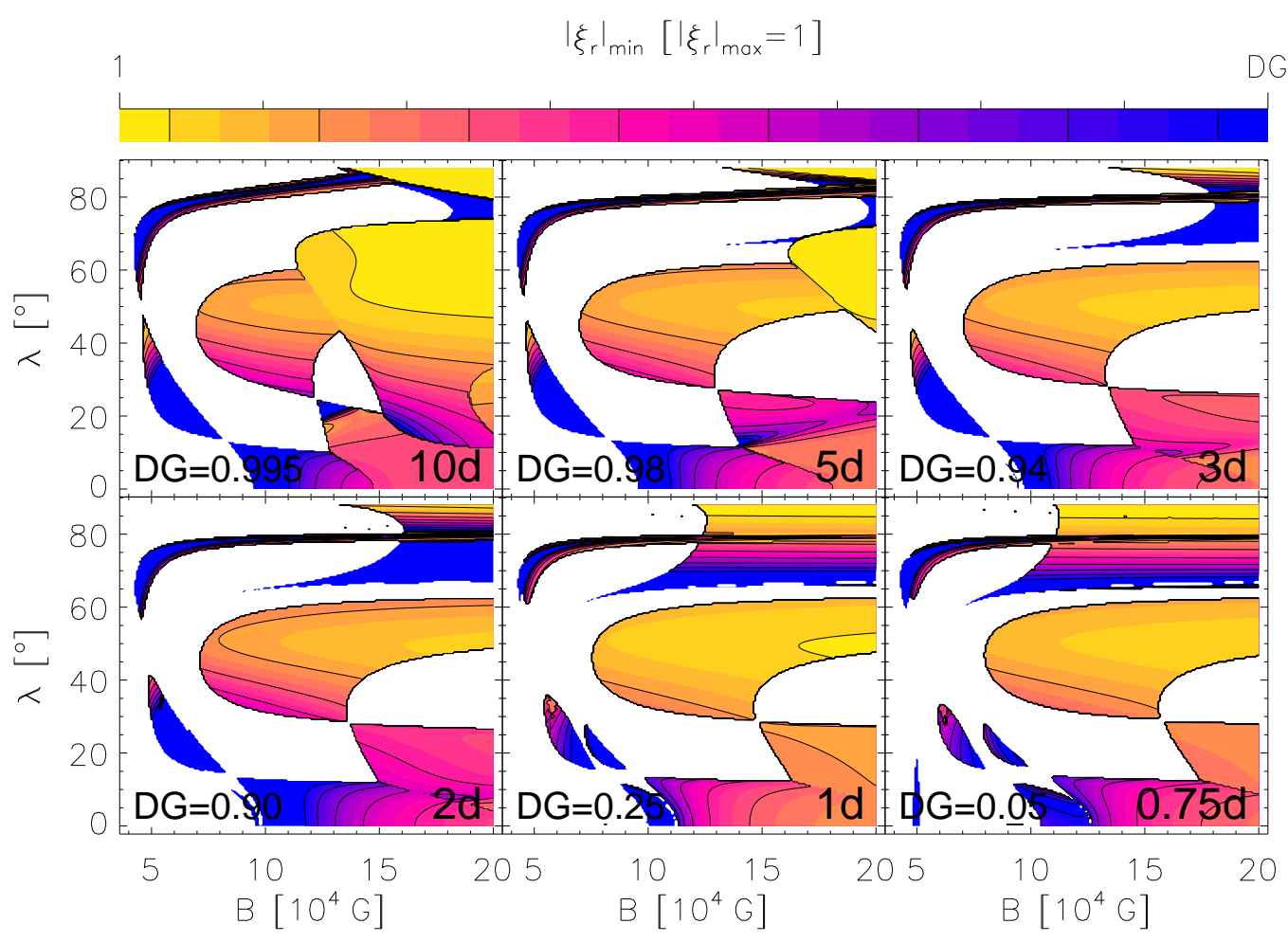

AbBILdung 9.20: Minima der radialen Enveloppe $\left|\hat{\xi}_{r}\right|_{\text {min }}$ als Funktion der Umlaufsdauer $T$. Die Darstellungsgrenze (DG) ist in jedem Diagramm verschieden um den Verlauf bei Instabilitäten mit kurzen Anwachszeiten aufzulösen; für $\left|\hat{\xi}_{r}\right|_{\min }<\mathrm{DG}$ wurde $\left|\hat{\xi}_{r}\right|_{\min }=\mathrm{DG}$ gesetzt. Instabilitäten mit $\tau \geq 10^{4} \mathrm{~d}$ werden nicht dargestellt. 


\subsection{Diskussion}

Die Brechung der Axialsymmetrie bezüglich der Rotationsachse durch den Begleiter führt in einem Doppelsternsystem zu weitreichenden Änderungen des Stabilitätsverhaltens magnetischer Flussringe. Die vom Begleitstern verursachte Gezeitenwirkung beeinflusst sowohl die Gleichgewichtskonfiguration als auch die Eigenmodi, die eine Antwort der Flussringe auf Störungen aus der umgebenden Konvektionszone darstellen.

Durch den Gezeiteneinfluss existiert entlang des ebenen Flussrings im stationären Kräftegleichgewicht eine periodische Variation der externen und internen Größen. Diese Variationen hängen im Rahmen der Näherung von der jeweiligen Skalenhöhe am Ort der Flussröhre ab. Da sich die Superadiabatizität $\delta$ in der Overshoot-Region am stärksten mit der Tiefe ändert und die Stabilitätseigenschaften empfindlich von dieser Größe abhängen, beeinflusst sie wesentlich die Reaktion einer Flussröhre auf Auslenkungen aus der toroidalen Gleichgewichtslage. Die Abhängigkeit von der Länge $\phi$ wirkt sich in komplizierter Form auf die Eigenfrequenzen $\omega$ und die Eigenfunktionen $\xi(\phi, t)$ aus. Dabei zeigt sich ein deutlicher Unterschied zwischen der Wirkung der schnellen Rotation und der periodischen Ortsabhängigkeit: Während die Rotation eine vergleichsweise deutliche Änderung der Eigenfrequenzen hervorruft und die Struktur der Eigenfunktionen nur unwesentlich beeinflusst, wirkt sich der Doppelsterncharakter in erster Linie auf die Ortsabhängigkeit der Eigenfunktionen aus, ohne die Frequenzen stark zu verändern. Die grundlegende Änderung der Eigenmodi entsteht durch die Kopplung eines ganzen Wellenspektrums mit den Wellenzahlen $m, m \pm 2, m \pm 4, \ldots$ zu einem Eigenmodus, was sich letztlich in einer periodischen Modulation der Auslenkung der Flussröhre äußert. Wegen der geringen Kopplung tragen aber nur wenige Partialwellen zu der resultierenden Gesamtform bei.

Sowohl die Ergebnisse des analytischen Sonderfalls einer Flussröhre in der Äquatorebene (Abschn. 9.3) als auch die numerische Untersuchung des allgemeinen Falls (Abschn. 9.4) zeigen, dass die Abweichungen der Eigenfrequenzen und -funktionen von denen des Einzelsternproblems i.Allg. zwar klein sind, aber die Größenordnungen der vom Begleitstern bewirkten, ursächlichen Störung deutlich übertreffen. Den Extremfall bilden die durch Kopplung zwischen benachbarten Partialwellen hervorgerufenen Resonanzinstabilitäten, die eine derart starke Änderung der Eigenfunktionen bewirken, dass es an den Maxima $\phi_{\max }$ und, wegen der Periodizität des Problems, $\phi_{\max }+\pi$ ihrer Enveloppe zu einer sehr deutlichen Bevorzugung der Entwicklung einer Instabilität kommt.

Die Stabilitätsanalyse erbrachte das interessante Ergebnis, dass diese Maxima bei stabilen Eigenmodi mit $\Im(\omega)=0$ entweder bei den Punkten $V_{1,2}$ an der Verbindungslinie oder bei den Quadraturpunkten $Q_{1,2}$ in senkrechter Richtung zur Verbindungslinie liegen. Bei instabilen Eigenmodi weisen die Maxima typischerweise eine Phasenverschiebung bezüglich dieser Punkte auf, sodass generell jede Länge $0 \leq \phi_{\max }<\pi$ möglich ist. Dabei hängt die Orientierung der Maxima in komplizierter Weise von den Gleichgewichtsparametern $\lambda$ und $B$ sowie dem dominierenden Wellenmodus $m$ ab. Die Maxima der äquatornahen $m=1$-Instabilitäten sind beispielsweise dicht bei $V_{1,2}$ um die Verbindungslinie gruppiert, die der $m=2$-Instabilitäten bei mittleren Breiten überdecken hingegen ein breiteres Intervall um die Quadraturpunkte. Das sich ergebende Bild ist in Abb. $9.21 \mathrm{zu}-$ sammenfassend dargestellt: Im Einzelsternproblem ist die Enveloppe einer Eigenfunktion grundsätzlich kreisförmig, im Doppelsternproblem hingegen deformiert. 

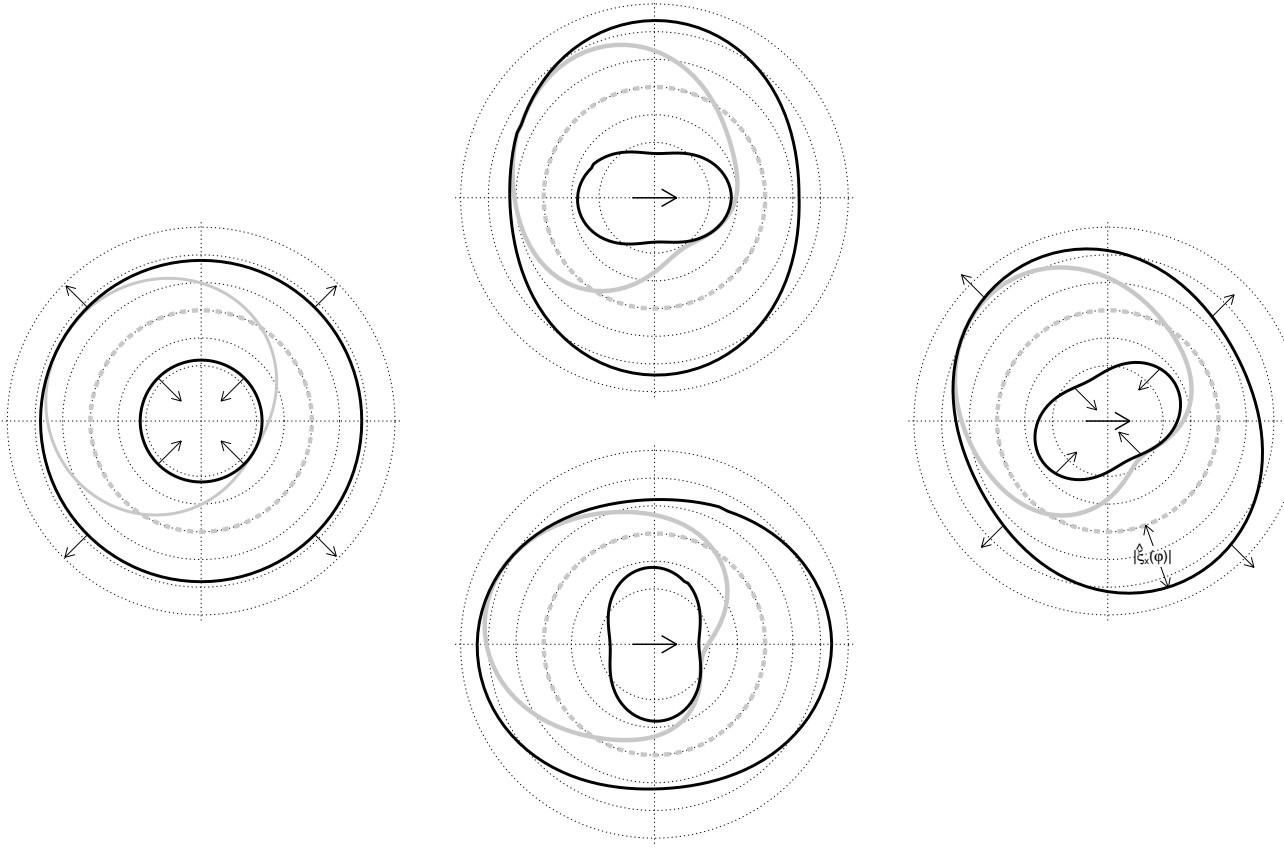

ABBILDUNG 9.21: Schematische Darstellung einer instabilen Flussröhre im Einzelstern (links) bzw. Doppelstern (rechts). In der Mitte sind zwei stabile Flussröhren in einem Doppelstern dargestellt, deren Enveloppemaxima entweder an den Quadraturpunkten $Q_{1,2}$ (oben) oder an der Verbindungslinie bei $V_{1,2}$ (unten) liegen. Bei der instabilen Flussröhre rechts haben die Maxima eine Phasenverschiebung bezüglich dieser Punkte.

Zur Beurteilung der ortsabhängigen Entstehungswahrscheinlichkeit von aufsteigenden Flussröhrenschleifen dient die Variation zwischen Enveloppe-Maximum und Minimum. Dies beruht auf der Überlegung, dass die Segmente der Flussröhre bei dem Einsetzen der Instabilität bevorzugt werden, bei denen die Auslenkung in Richtung der Konvektionszone am größten ist. Die Enveloppe bestimmt die Maximalauslenkung beim Phasendurchgang eines Wellenberges, und da diese bei den Längen $\phi_{\max }$ etwas größer ist als bei den Minima ist auch hier die Wahrscheinlichkeit eines Aufstiegs etwas größer. Da es sich dabei grundsätzlich um eine statistische Aussage handelt, ist es nicht möglich den Entstehungsort von aufsteigenden Schleifen einer individuellen Flussröhre zu bestimmen. Die Tendenz zum Ausbruch bei den Vorzugslängen macht sich erst bei der Betrachtung eines ausreichend großen Ensembles von vergleichbaren Flussröhren über einen längeren Zeitraum hinweg bemerkbar. Dies setzt voraus, dass sich die Umgebungsbedingungen, z.B. die Art und Stärke der überschießenden Gaspakete aus der Konvektionszone als potenzielle Ursache der Gleichgewichtsstörungen, nicht wesentlich ändern und sowohl im zeitlichen als auch räumlichen Mittel konstant sind. Diese Annahme schließt gegenwärtig die Beschreibung zeitabhängiger Phänomene, wie z.B. die Migration von Vorzugslängen, im Rahmen dieses Modells aus.

Im Falle von engen Doppelsternsystemen mit Rotationsdauern von wenigen Tagen kann das Enveloppe-Minimum von auftriebsbedingten Instabilitäten um bis zu $20 \%$ klei- 
ner als das Maximum sein. Aus Sicht der linearen Stabilitätsanalyse existieren somit tatsächlich Vorzugslängen bei der Bildung von instabilen, aufsteigenden Flussröhrensegmenten, wobei die Orientierung und Ausprägung der Bevorzugung vom jeweiligen Parameterbereich $(B, \lambda)$ der Gleichgewichtskonfiguration abhängt. Und wie die Parameterstudie in Abschn. 9.4.3 zeigt, ist die Rotationsperiode von $T \lesssim 5 \ldots 10 \mathrm{~d}$ für das Einsetzen von ausgeprägten Vorzugslängen konsistent mit den Beobachtungen. Instabilitäten bei kleineren Feldstärken und insbesondere Resonanzinstabilitäten weisen dabei die ausgeprägtesten Vorzugslängen auf. Allerdings sind deren Anwachszeiten ein Vielfaches von dem was frühere Arbeiten im Fall der Sonne erwarten lassen würden. Jedoch in Hinblick auf die Tatsache, dass es sich bei den beobachteten Vorzugslängen an der Oberfläche aktiver Doppelsterne um ein vergleichsweise schwach ausgeprägtes Phänomen handelt, kann der Einfluss solcher Sekundäreffekte a priori nicht ausgeschlossen werden.

Vorzugslängen bei der Entstehung aufsteigender Flussschleifen am unteren Rand der Konvektionszone entsprechen nicht notwendigerweise denen, die man an der Oberfläche eines aktiven Sterns beobachtet. Es ist a priori auch nicht zu erwarten, dass diese in einem einfachen Zusammenhang stehen, da Flussröhren während ihres Aufstiegs weiterhin dem längenabhängigen Gezeiteneinfluss unterliegen. Die lineare Analyse zeigt lediglich an, wie, wo und in welchem Ausmaß sich der Doppelsterncharakter auf das Stabilitätsverhalten magnetischer Flussröhren in der Overshoot-Region auswirkt ${ }^{9}$. Die Bestimmung der Ausbruchsregionen an der Sternoberfläche ist Gegenstand der nicht-linearen Entwicklungssimulationen in Kap. 10.

\footnotetext{
${ }^{9}$ Würde die Flussröhren-Entwicklung durch die gesamte Konvektionszone streng linear verlaufen, so würde die Orientierung der Enveloppe-Maxima die Lage von Vorzugslängen auch an der Oberfläche anzeigen.
} 


\section{Kapitel 10}

\section{Simulationen}

\subsection{Verfahren}

\subsubsection{Implementierung des Doppelsterncharakters}

Die Erweiterung des Flussröhren-Codes auf Doppelsterne umfasst die vom Begleitstern hervorgerufene Gezeitenkraft g $_{\text {tide }}$ sowie die aus ihr resultierende Deformation des Sternkörpers. Die Gezeitenbeschleunigung hat die Form

$$
\mathbf{g}_{\text {tide }}=g_{*}(r) \sigma q \epsilon^{3}\left[3\left(\mathbf{e}_{r} \cdot \mathbf{e}_{a}\right) \mathbf{e}_{a}-\mathbf{e}_{r}\right]+\mathcal{O}\left(\epsilon^{4}\right) .
$$

Die Gravitationsbeschleunigung $g_{*}(r)$ wird einem tabellarisierten Sternmodell entnommen, die Größen $\epsilon, q, \sigma, \mathbf{e}_{r}$ und $\mathbf{e}_{a}$ wurden in Abschn. 8.1.1 definiert. Zusammen mit der Zentrifugalbeschleunigung ergibt sich daraus die in Gl. (8.13) angegebene effektive Gravitation $g_{\text {eff }}$.

In dem verwendeten numerischen Verfahren wird die Sternschichtung am Ort eines Massenelements der Flussröhre anhand dessen Abstands vom Sternmittelpunkt aus einem sphärisch-symmetrischen Sternmodell bestimmt. Bei der Untersuchung des Doppelsternproblems wird nun für jedes dieser Massenelemente am jeweiligen Ort die geometrische Abweichung der Äquipotenzialfläche von der Kugelform mittels der Radiuskorrektur $\delta r(r, \phi, \lambda)$ nach Gl. (8.22) berechnet und bei der anschließenden Bestimmung der externen Schichtung aus dem tabellarisierten Modell berücksichtigt. Bei dieser Methode umgeht man, im Gegensatz zur Gl. (8.19), die Berechnung der Skalenhöhen entlang der Flussröhre und die Limitierung (8.28) der Näherung. Vor der Berechnung von $\delta r$ erfolgt die Bestimmung des Faktors $\sigma=M_{*} / M(r)$ unter der Annahme einer kugelsymmetrischen Massenverteilung. Für das im Referenzfall verwendete Sternmodell (der heutigen Sonne) gilt innerhalb der Konvektionszone in guter Näherung $\sigma \simeq 1$ (vgl. Abb. 8.4).

\subsubsection{Ausgangssituation in der Overshoot-Region}

Ausgangspunkt der nicht-linearen Rechnungen sind toroidale isentrope Flussröhren in der Mitte der Overshoot-Region am unteren Rand der Konvektionszone (vgl. Tab. 9.1). Da die in Abschn. 9.1 beschriebene Entropievariation des analytischen Gleichgewichts vernachlässigbar ist, können die Ergebnisse der linearen Stabilitätsanalyse aus Abschn. 9.4 


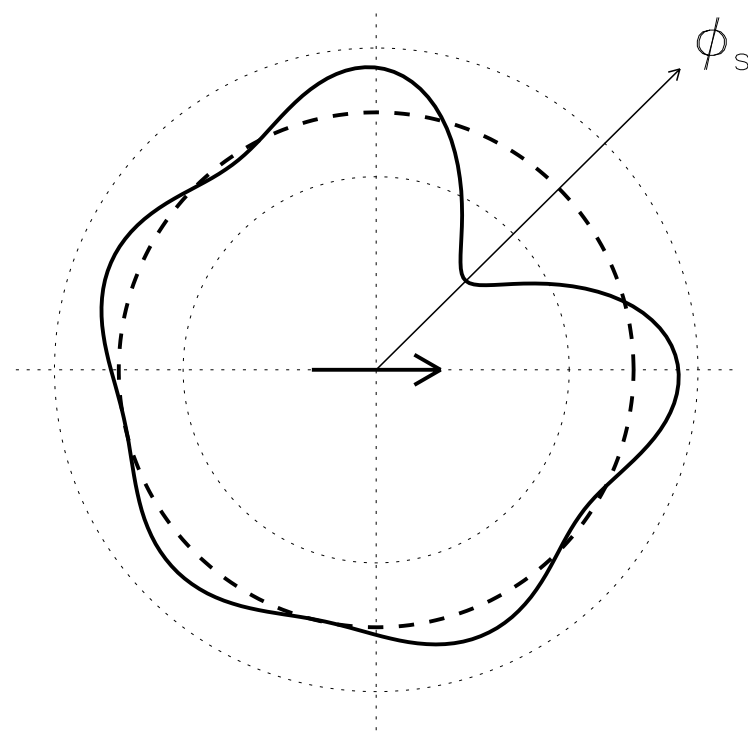

ABBILDUNG 10.1: Schematische Darstellung der Störung einer toroidalen Flussröhre (gestrichelte Linie) mittels einer Superposition harmonischer Wellen der azimutalen Wellenzahlen $m=1 \ldots 5$ (durchgezogene Linie). Durch phasengleiche Überlagerung entsteht eine Lokalisierung der Störung um den Phasenwinkel $\phi_{\mathrm{s}}$, die spezifische Störungslänge. Die Richtung zum Begleitstern bei $\phi=0$ wird durch den zentralen Pfeil verdeutlicht.

als Anhaltspunkt für die Anwachszeiten und die Wahl des zu untersuchenden Parameterbereichs $(B, \lambda)$ verwendet werden. Für die Startkonfigurationen, im Weiteren mit dem Index ' $s$ ' gekennzeichnet, werden magnetische Feldstärken im Bereich $B_{\mathrm{s}}=7 \ldots 20 \cdot 10^{4} \mathrm{G}$ gewählt. Die kurzen Anwachszeiten in diesem Intervall lassen erwarten, dass dem Dynamoprozess nicht genügend Zeit bleibt um Magnetfelder noch auf wesentlich höhere Feldstärken zu verstärken, bevor die Flussröhren ausbrechen. Die Stabilitätsanalyse ergab zwar bei mittleren Breiten (zwischen $\lambda_{\mathrm{s}}=30 \ldots 45^{\circ}$ ) eine Stabilitätsinsel, welche die Speicherung über lange Zeiträume auch bei hohen Feldstärken zu ermöglichen scheint. Wie die Simulationen aber zeigen, kommt es aufgrund einer zusätzlichen Instabilität, die im Rahmen der linearen Analyse nicht berücksichtigt werden kann, hier dennoch zu Ausbrüchen auf vergleichsweise kurzen Zeitskalen. Auf diese durch Reibungseffekte ausgelöste Instabilität wird in Kap. 14 genauer eingegangen.

Das Ziel der Simulationen ist die Untersuchung des Einflusses eines Begleitsterns auf die Oberflächenverteilung ausbrechender Flussröhren. Während diese im Einzelsternproblem aufgrund der Rotationssymmetrie nur eine Funktion der Breite $\lambda$ ist, wird in einem Doppelstern durch die ausgezeichnete Richtung zum Begleiter eine Abhängigkeit von der Länge $\phi$ in das Problem eingeführt. Diesem azimutalen Freiheitsgrad muss auch in der Ausgangssituation innerhalb der Overshoot-Region Rechnung getragen werden. In den Simulationen geschieht dies mittels der azimutalen Verschiebung einer lokalisierten Störung der toroidalen Flussröhre um den Stern. Die Anregung einer Instabilität erfolgt durch die Superposition harmonischer Wellen der azimutalen Wellenzahlen $m=1 \ldots 5$ und Amplituden von etwa $0.01 H_{p}$. Phasengleiche Überlagerung bewirkt eine Lokalisierung der Auslenkung in einer Umgebung der spezifischen Störungslänge $\phi_{\mathrm{s}}$ (Abb. 10.1); als physikalischer Hintergrund für diesen Ansatz diene das Bild der Auslenkung aufgrund eines aus der Konvektionszone überschießenden Gaselements. Um den Einfluss des Begleiters auf die Flussröhrenentwicklung adäquat aufzulösen, ist ein gutes Abtasten der Längenabhängigkeit notwendig. Dies geschieht mittels 20 Rechnungen aufsteigender Flussröhren, deren Störungszentren sukzessive um jeweils $\Delta \phi_{\mathrm{s}}=9^{\circ}$ verschoben werden $^{1}$.

\footnotetext{
${ }^{1}$ Wegen der azimutalen $\pi$-Periodizität des Doppelsternproblems wird grundsätzlich nur das Längenin-
} 
Das Abtasten der Breitenabhängigkeit erfolgt in Schritten von $5^{\circ}$ zwischen den Startbreiten $\lambda_{\mathrm{s}}=0 \ldots 80^{\circ}$. Um den ortsabhängigen Einfluss des Begleitsterns zu analysieren werden somit pro Feldstärkewert $B_{\mathrm{s}}$ insgesamt $20 \times 17=340$ Flussröhren betrachtet, von denen jede ein $9^{\circ} \times 5^{\circ}$ großes Winkelareal repräsentiert.

Das oben beschriebene Winkelraster repräsentiert eine umfassende Abdeckung möglicher Störungsorte magnetischer Flussröhren in der Overshoot-Region. Die nicht-linearen Rechnungen verfolgen deren Aufstieg zur Oberfläche (sofern sie durch die Störung instabil werden) und entsprechen der Abbildung einer gleichmäßigen Ausgangsverteilung von Flussröhren und Störungen am unteren Rand der Konvektionszone auf die Oberfläche. Diese entspricht aber nicht notwendigerweise den zu erwartenden beobachtbaren Fleckenverteilungen. Dazu wären zusätzliche Angaben über die tatsächliche Situation in der Overshoot-Region notwendig, wie z.B. die Kenntnis, bei welcher Breite wieviele Flussröhren mit welchen Feldstärken vorhanden sind. Derartige Verteilungen und Eigenschaften werden u.a. vom zugrundeliegenden Dynamomechanismus bestimmt und könnten anhand entsprechender Theorien vorhergesagt werden. Im Weiteren wird keine Annahme bezüglich der räumlichen oder zeitlichen Verteilung magnetischer Flussröhren in der Overshoot-Region gemacht, sondern grundsätzlich davon ausgegangen, dass jede der untersuchten Parameterkonfigurationen eine potenzielle Ausgangssituation darstellt. In diesem Sinne liefert die Abbildung der Ausgangsverteilung auf die Oberfläche Hinweise auf zu erwartende Ausbruchsorte, falls entsprechende Flussringe in der Overshoot-Region vorhanden sind.

\subsection{Ergebnisse im Referenzfall}

Die Rechnungen simulieren die Entwicklung magnetischer Flussröhren über einen Zeitraum von maximal $10^{9} \mathrm{~s}(\approx 32 \mathrm{yr})$ nach Störung der toroidalen Ausgangskonfiguration. Flussröhren, die innerhalb dieses Zeitraums nicht an der Oberfläche ausbrechen, werden in den Verteilungsfunktionen nicht berücksichtigt. Größen, die sich auf den Ausbruch beziehen, werden mit dem Index ' $a$ ' gekennzeichnet.

\subsubsection{Ausbruchszeiten}

Die Abhängigkeit der Ausbruchszeit $t_{\mathrm{a}}$ von der Startbreite $\lambda_{\mathrm{s}}$ und Anfangsfeldstärke $B_{\mathrm{s}}$ spiegelt in groben Zügen die Eigenschaften der Instabilitäten am unteren Rand der Konvektionszone wider (Abb. 10.2). Im Bereich der Parker-Instabilitäten liegen sie typischerweise bei wenigen Jahren, in der Nähe der Instabilitätsgrenzen sind sie erwartungsgemäß etwas größer. Zu höheren Feldstärken hin nimmt $t_{\mathrm{a}}$ aufgrund des stärkeren magnetischen Auftriebs deutlich ab. Simulationen in den Instabilitätsinseln bei $B_{\mathrm{s}} \simeq 6 \cdot 10^{4} \mathrm{G}$ und $\lambda_{\mathrm{s}} \simeq 20^{\circ}$ ergeben Ausbruchszeiten in der Größenordnung von mehreren hundert Jahren; weitere Untersuchung in diesem Gebiet sind wenig sinnvoll. Darüber hinaus ergeben sich einige Besonderheiten, die aus der linearen Stabilitätsanalyse nicht ersichtlich sind. In einzelnen Bereichen kommt es zu Ausbrüchen, obwohl die lineare Analyse in

tervall $\phi_{\mathrm{s}}=0 \ldots 180^{\circ}$ untersucht. 


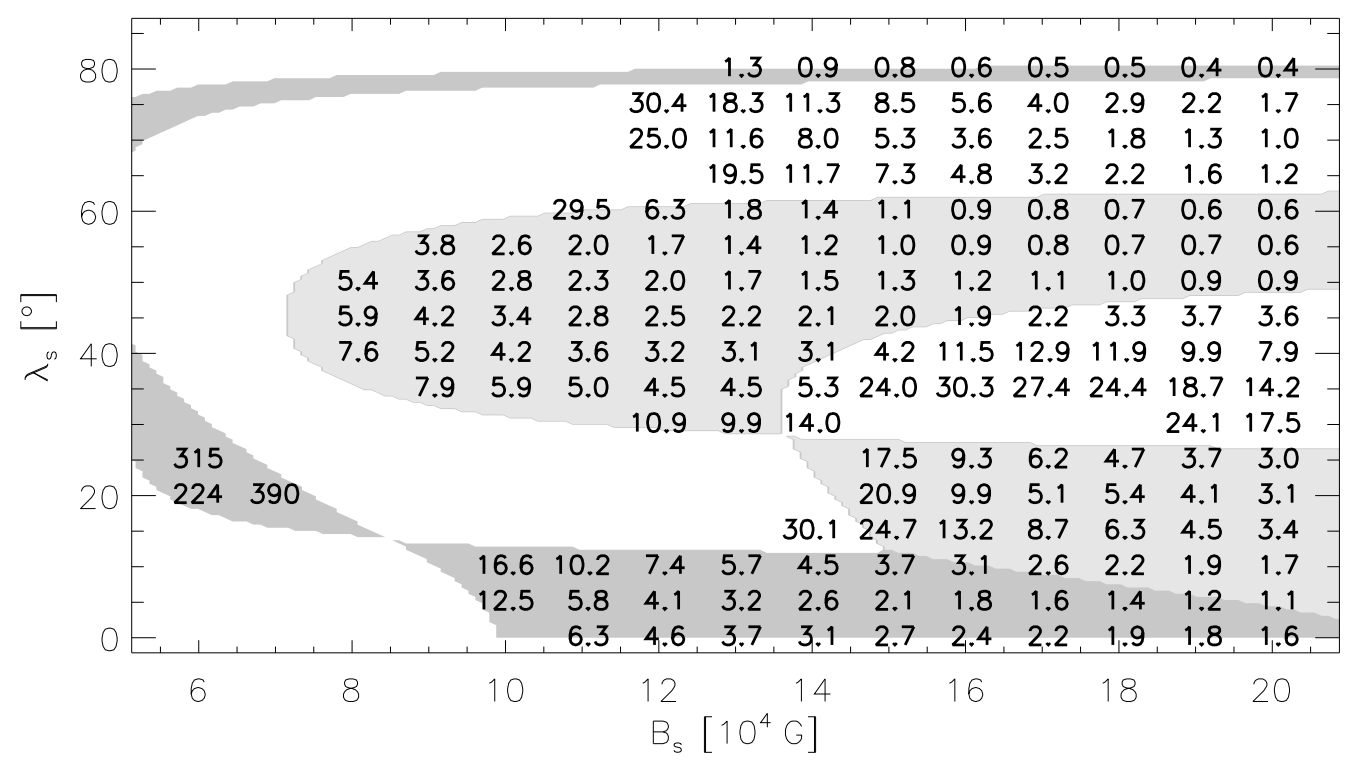

AbBildung 10.2: Ausbruchszeiten $t_{\mathrm{a}}$ (in Jahren). Die Positionen im Diagramm beziehen sich auf die Ausgangskonfiguration am unteren Rand der Konvektionszone und stellen keine Breitenverteilung an der Oberfläche dar. Bereiche der Parker-Instabilität sind grau unterlegt (dunkel: azimutale Wellenzahl $m=1$, hell: $m=2$ ).

Abschn. 9.4 dort Quasi-Stabilität (mit Anwachszeiten von etlichen $10^{4} \mathrm{~d}$ voraussagt), beispielsweise in der Stabilitätsinsel bei mittleren Breiten und hohen Feldstärken oder in dem schmalen Bereich oberhalb $60^{\circ}$ Breite. Die Ausbruchszeiten sind hier zum überwiegenden Teil so kurz, dass sie im Widerspruch zu den sehr langen Anwachszeiten $\tau$ stehen und daher nicht durch die Doppelstern-typischen Instabilitätsplateaus hervorgerufen werden (siehe Abschn. 10.3). Eine eingehendere Untersuchung dieser Erscheinung führte auf die Entdeckung einer durch Reibung ausgelösten Instabilität, deren Ursache im aerodynamischen Widerstand einer sich relativ zum umgebenden Medium bewegenden Flussröhre liegt. Der qualitative Verlauf ihrer Anwachszeiten (Abb. 13.1) ist konsistent mit den hier auftretenden Ausbruchszeiten.

Der Aufstieg magnetischer Flussröhren erfolgt nicht immer kontinuierlich. Bei Flussröhren mit Startbreiten $\lambda_{\mathrm{s}}=15 \ldots 35^{\circ}$, die oberhalb $B_{\mathrm{s}} \gtrsim 15 \cdot 10^{4} \mathrm{G}$ instabil werden, kommt die Aufwärtsbewegung im unteren Teil der Konvektionszone oft zeitweise zum Erliegen und setzt sich erst nach einigen hundert Tagen wieder fort. Die resultierende zeitliche Verzögerung ist in Abb. 10.2 zu erkennen. Obwohl die linearen Anwachszeiten in der Nähe des Äquators mit denen der bei etwas höheren Breiten liegenden Flussröhren vergleichbar sind, ergeben sich für deren Ausbrüche deutlich längere Zeiten $t_{\mathrm{a}}$. Der genaue Grund für dieses Verhalten ist unklar.

\subsubsection{Breitenverteilungen}

Während bei der Sonne dunkle Flecken nur in einem äquatorialen Band von $\pm 35^{\circ}$ Breite $\mathrm{zu}$ beobachten sind, zeigen schnell rotierende Sterne typischerweise auch Flecken bei 


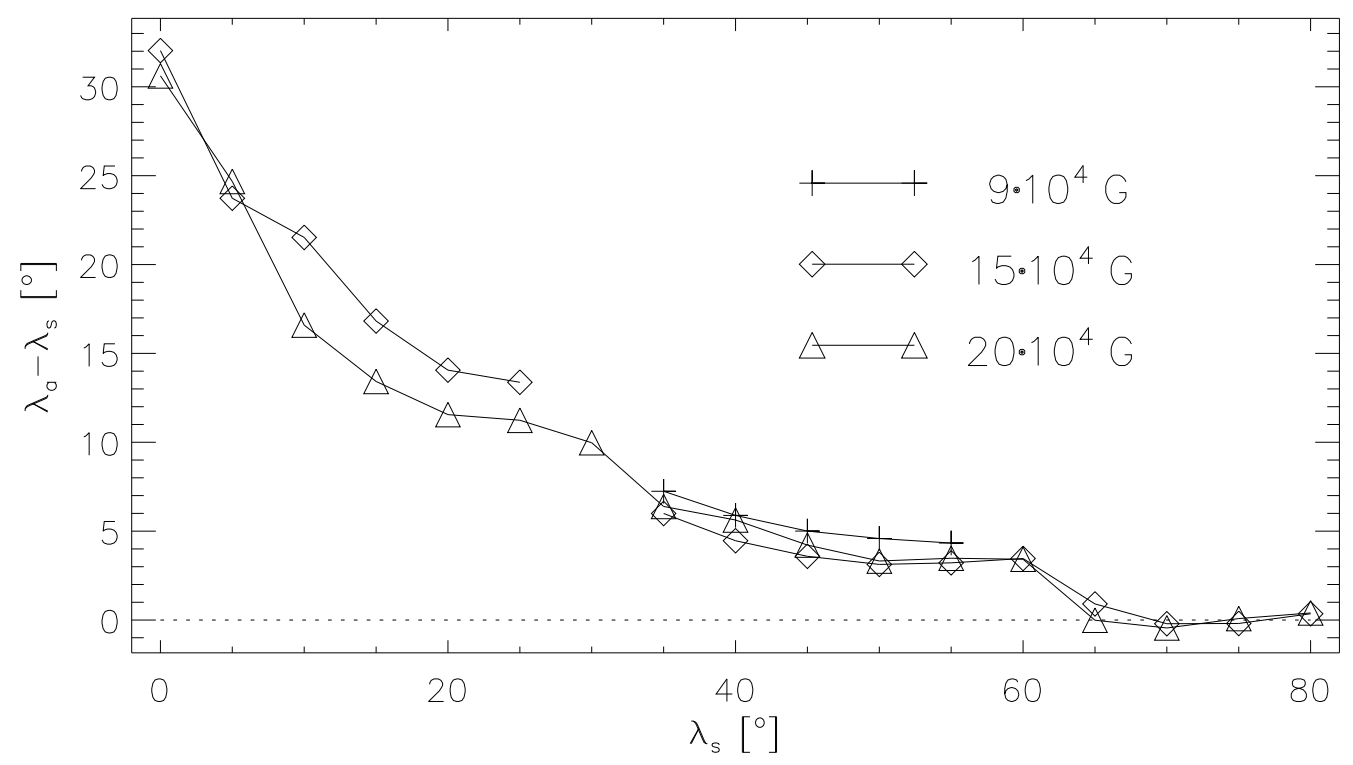

ABBILDUNG 10.3: Polwärtige Ablenkung $\left(\lambda_{\mathrm{a}}-\lambda_{\mathrm{s}}\right)$ ausbrechender Flussröhren als Funktion der Startbreite $\lambda_{\mathrm{s}}$ in der Overshoot-Region. Die Symbole markieren verschiedene Feldstärken; bei $B_{\mathrm{s}}=9 \cdot 10^{4} \mathrm{G}$ erfolgt nur für Flussröhren mit mittleren Startbreiten ein Ausbruch (vgl. Abb. 10.2). Angegeben werden über alle Störungslängen $\phi_{\mathrm{s}}$ gemittelte Werte.

mittleren und polaren Breiten. Diese Erscheinung wird im Rahmen des Flussröhrenmodells durch die polwärts gerichtete Ablenkung aufsteigender Flussröhren aufgrund der Corioliskraft erklärt (Schüssler \& Solanki 1992). Abbildung 10.3 zeigt die polwärtige Verschiebung der Ausbruchsbreite $\lambda_{\mathrm{a}}$ als Funktion der Startbreite $\lambda_{\mathrm{s}}$ für verschiedene Feldstärken $B_{\mathrm{s}}$. Bei niedrigen Startbreiten $\left(\lambda_{\mathrm{s}} \lesssim 30^{\circ}\right)$ ist die Ablenkung am gravierendsten, sodass in schnellen Rotatoren äquatornahe Breiten weitgehend fleckenfrei sein sollten. Dies steht jedoch im Widerspruch zu Beobachtungen, bei denen z.T. auch bei niederen Breiten Flecken gefunden werden. Deren Existenz ist im Rahmen des Flussröhrenmodells nur mittels Transport durch Oberflächenströmungen zu erklären. Die Ablenkung nimmt mit der Startbreite deutlich ab. In mittleren Breiten beträgt sie nur noch wenige Grad und bei höheren Breiten und am Pol tritt praktisch gar keine Ablenkung mehr auf. Oberhalb $60^{\circ}$ scheinen Flussröhren ungefähr bei den Breiten auszubrechen die ihren ursprünglichen Startbreiten innerhalb der Overshoot-Region entsprechen. Dies würde bedeuten, dass polare Flecken durch einen Dynamomechanismus bei sehr hohen Breiten erzeugt werden. Da bei den Simulationen jedoch keine meridionale Strömungen innerhalb der Konvektionszone berücksichtigt wurden, ist nicht auszuschließen, dass Flecken möglicherweise bei niedrigeren Breiten ausbrechen und erst danach Richtung Pol driften; diese Möglichkeit wird auch von Schrijver \& Title (2001) in Betracht gezogen. Insbesondere bei kleinen Breiten nimmt die Ablenkung mit wachsender Feldstärke ab, da der größere magnetische Auftrieb den Aufstieg in sphärisch-radialer Richtung stärker vorantreibt. 


\subsubsection{Längenverteilungen}

\section{Zur Analyse des Einflusses des Begleitsterns}

Abbildung 10.4 zeigt exemplarische Längenverteilungen von Ausbruchsorten $\phi_{\mathrm{a}}$ als Funktion der Störungslänge $\phi_{\mathrm{s}}$; die Fälle unterscheiden sich in den Startparametern $\left(B_{\mathrm{s}}, \lambda_{\mathrm{s}}\right)$. Generell bricht eine Flussröhre wegen des Wellencharakters der anfänglichen Instabilität und der asymmetrischen Entwicklung in den beiden Flanken der aufsteigenden Schleife nicht bei der Störungslänge $\phi_{\mathrm{s}}$, sondern bei einer anderen Länge $\phi_{\mathrm{a}}$ aus. Verschiebt man in einem Einzelstern die Störungslänge um $\Delta \phi_{\mathrm{s}}$, so wird die Entwicklung wegen der Axialsymmetrie des Sterns nur zu einer entsprechenden Verschiebung $\Delta \phi_{\mathrm{a}}=\Delta \phi_{\mathrm{s}}$ des Ausbruchsorts führen. Dieser einfache Sachverhalt wird in Abb. 10.4 durch gepunktete Linien veranschaulicht. In einem deformierten Doppelstern existieren hingegen längenabhängige Umgebungsbedingungen und Gezeitenkräfte, welche die Entwicklung der Flussröhre während des Aufstiegs beeinflussen und sich bis zur Oberfläche zu einer von $\phi_{\mathrm{s}}$ abhängigen Verschiebung des Ausbruchsortes aufsummieren. Die Ausbruchslängen $\phi_{a}$, in Abb. 10.4 durch Kreuze gekennzeichnet, weichen folglich von denen des entsprechenden Einzelsternproblems ab. Die Stetigkeit und Periodizität der nichtlinearen Funktion $\phi_{\mathrm{a}}\left(\phi_{\mathrm{s}}\right)$ erlaubt die Interpolation weiterer Ausbruchslängen und deren anschließende Zusammenfassung in einem Histogramm. Diese Verteilungsfunktion gibt Hinweise auf die Existenz und Lage von Häufungspunkten, die anzeigen, ob und bei welchen Längen Flussröhren vermehrt die Oberfläche erreichen. Im oberen Diagramm der Abb. 10.4 ist die Abweichung vom linearen Verlauf eines Einzelsterns gering, weshalb die resultierende Verteilung nur eine eher unauffällige Verringerung von Ausbrüchen in der Umgebung der Quadraturpunkte und eine entsprechend schwache Erhöhung um die Verbindungslinie zeigt. Im mittleren Diagramm ist die Abweichung deutlich stärker und bewirkt einen sehr ausgeprägten Häufungspunkt am Quadraturpunkt. Im unten dargestellten Fall ergeben sich hingegen zwei Häufungspunkte, die ein Intervall bevorzugter Ausbruchslängen von einem trennen, welches offenbar gemieden wird. Der Verlauf von $\phi_{\mathrm{a}}\left(\phi_{\mathrm{s}}\right)$ ist nicht bei allen Ausgangskonfigurationen stetig und die daraus resultierenden Verteilungsfunktionen so klar strukturiert wie in Abb. 10.4; auf diese Sonderfälle wird weiter unten in Abschn. 10.2.4 genauer eingegangen.

\section{Mittlere Startbreiten $\left(\lambda_{\mathrm{s}}=30 \ldots 60^{\circ}\right)$}

Am auffälligsten zeigt sich die Wirkung des Begleitsterns bei Flussröhren mit mittleren Startbreiten, die aufgrund der Parker-Instabilität mit azimutaler Wellenzahl $m=2$ ausbrechen (Abb. 10.5). Hier findet man für alle Ausgangskonfigurationen Vorzugslängen, die eine systematische Abhängigkeit von der Feldstärke $B_{\mathrm{s}}$ und Breite $\lambda_{\mathrm{s}}$ erkennen lassen. Ihre Ausprägung nimmt zu höheren Startbreiten hin deutlich ab. Am markantesten sind sie bei $\lambda_{\mathrm{s}}=35 \ldots 40^{\circ}$, wo ein Großteil aller Flussröhren innerhalb Längenintervallen von etwa $20 \ldots 30^{\circ}$ ausbricht. Teilweise treten so starke Konzentrationen auf, dass über ein Drittel — in Einzelfällen sogar fast die Hälfte — aller Flussröhren innerhalb eines nur $9^{\circ}$ umfassenden Längenintervalls lokalisiert sind. Zu höheren Startbreiten hin weichen diese lokalisierten Vorzugslängen zusehends auf und nehmen ein größeres Intervall ein, wobei die Häufungen aber nach wie vor signifikant sind. 


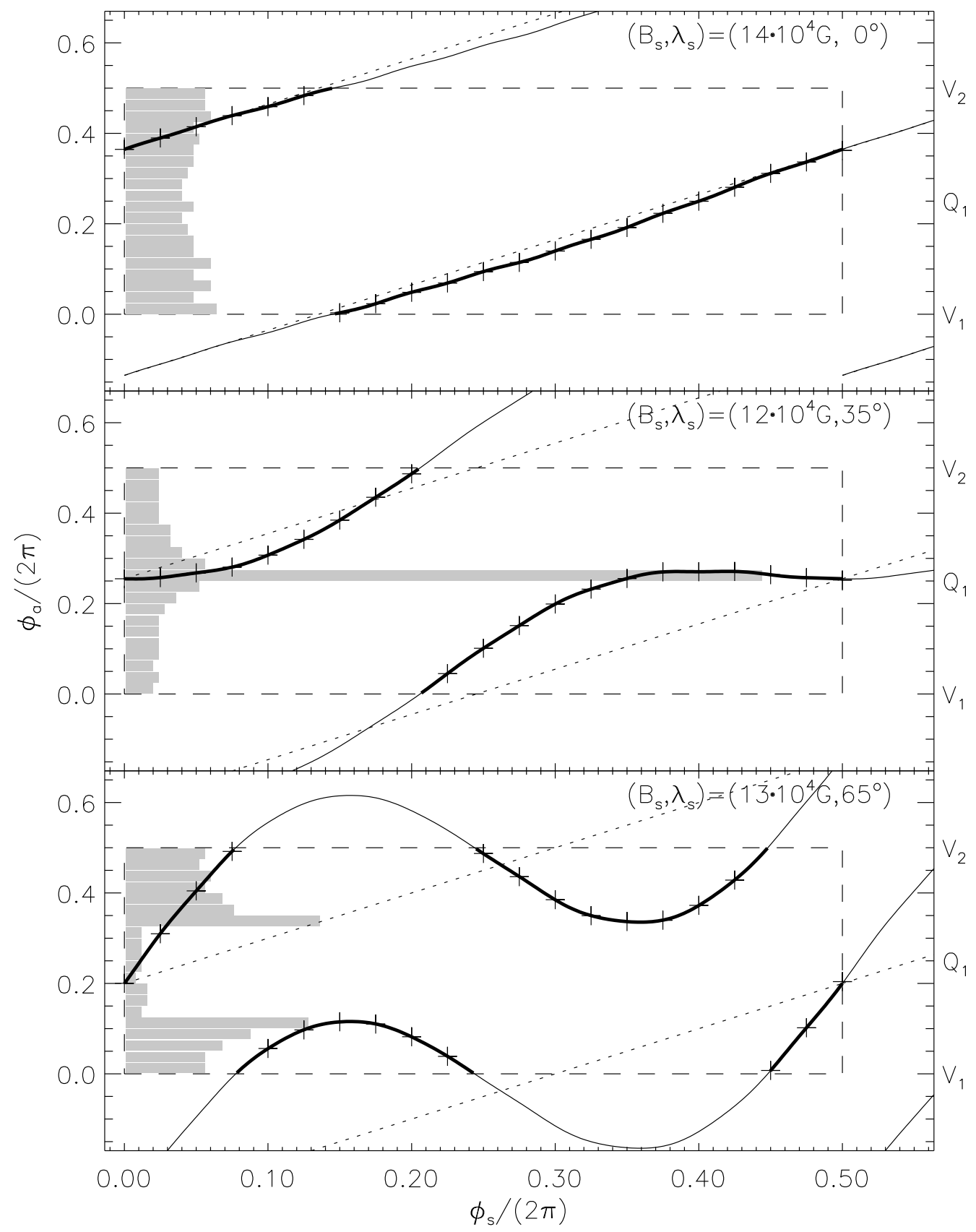

ABBILDUNG 10.4: Beispiele für Verteilungen von Ausbruchslängen $\phi_{\mathrm{a}}$ als Funktion der Störungslänge $\phi_{\mathrm{s}}$. Kreuze markieren gerechnete Flussröhrenentwicklungen, durchgezogene Linien den interpolierten, nicht-linearen Verlauf $\phi_{\mathrm{a}}\left(\phi_{\mathrm{s}}\right)$ beim Doppelsternproblem und gepunktete Linien den linearen Verlauf des trivialen Einzelsternproblems. Relevant ist nur die gestrichelt umrandete Zone, die umliegenden Bereiche sind periodische Fortsetzungen dieses Gebiets. Das grau unterlegte Histogramm gibt die normierte Verteilung der Ausbruchslängen in $9^{\circ}$ breiten $\phi_{\mathrm{a}^{-}}$ Intervallen wieder (es gelten die Zahlenwerte auf der Abszisse). 


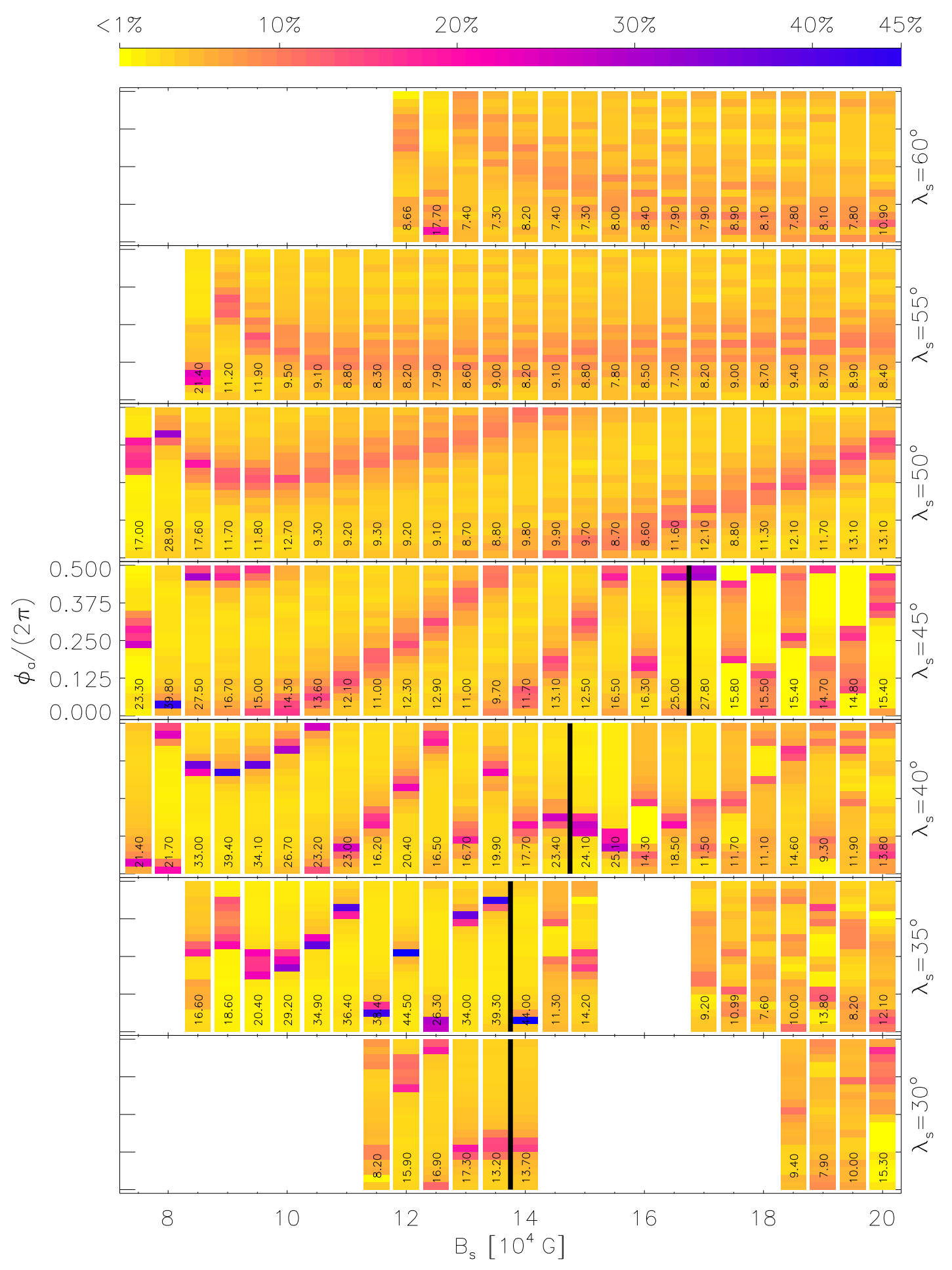

ABBILDUNG 10.5: Längenverteilungen ausbrechender Flussröhren mit mittleren Startbreiten $\lambda_{\mathrm{s}}=30 \ldots 60^{\circ}$. Die Farbkodierung am oberen Rand gibt den Anteil aller bei einer $\left(B_{\mathrm{s}}, \lambda_{\mathrm{s}}\right)$ Konfiguration startenden Flussröhren an, die im jeweiligen $9^{\circ}$ umfassenden $\phi_{\mathrm{a}}$-Intervall ausbrechen; die bei jeder Konfiguration angegebene Zahl den entsprechenden maximalen Prozentwert. Schwarze, vertikale Linien markieren die Grenze zwischen Parker- und Strömungsinstabilität. Die Verteilungen bei $\lambda_{\mathrm{s}}=30 \ldots 35^{\circ}$ und $B_{\mathrm{S}} \gtrsim 17 \cdot 10^{4} \mathrm{G}$ gehören zu den Sonderfällen. 
Eine wichtige Frage ist die nach der Phasenlage von Vorzugslängen in Bezug auf den Begleitstern bei $\phi=0$. Anhand Abb. 10.5 ist zu erkennen, dass grundsätzlich alle Orientierungen $\phi_{\mathrm{a}}$, in Abhängigkeit von der Ausgangskonfiguration $\left(B_{\mathrm{s}}, \lambda_{\mathrm{s}}\right)$ in der OvershootRegion in Frage kommen können . Für Feldstärken etwas oberhalb der Instabilitätsgrenze tritt typischerweise zuerst eine Verschiebung der Häufungspunkte zu kleineren Längen $\phi_{\mathrm{a}}$ auf, d.h. entgegen dem Rotationssinn des Sterns. Bei höheren Feldstärken kehrt sich dieser Trend i.Allg. um und die Verschiebung erfolgt mit zunehmender Feldstärke im Rotationssinn. Die Ausprägung dieses Verhaltens ist deutlich breitenabhängig. Bei niedrigen Startbreiten wandern Häufungspunkte bereits bei einer Änderung von $\Delta B_{\mathrm{s}} \sim 5 \cdot 10^{3} \mathrm{G}$ etwa um die halbe Hemisphäre, während bei höheren Breiten die Magnetfeldabhängigkeit wesentlich kleiner ist und für $\lambda_{\mathrm{s}}=60^{\circ}$ praktisch verschwindet; bei diesen Startbreiten scheinen Vorzugslängen am ehesten bezüglich der Lage des Begleitsterns fixiert zu sein. Die Verschiebung hängt offenbar mit der Ausprägung der Häufungspunkte zusammen: Die markantesten bei $\lambda_{\mathrm{s}}=35 \ldots 45^{\circ}$ zeigen eine empfindliche Abhängigkeit ihrer Orientierung von der Feldstärke, die schwächeren bei $\lambda_{\mathrm{s}} \simeq 60^{\circ}$ dagegen nur eine minimale, wenn überhaupt.

Aufgrund der Strömungsinstabilität brechen Flussröhren auch in Bereichen aus, die hinsichtlich der Parker-Instabilität stabil sind, wie z.B. für $B_{\mathrm{s}} \gtrsim 16 \cdot 10^{4} \mathrm{G}$ bei $40 \ldots 45^{\circ}$ Startbreite. Deren Verteilungsfunktionen unterscheiden sich qualitativ deutlich von denen benachbarter 'Parker-instabilen' Ausgangskonfigurationen. Sie werden überwiegend von ausgedehnten Vorzugsintervallen dominiert, bei denen weniger die Bevorzugung als vielmehr die Meidung bestimmter Längen auffällt. Eine übergeordnete Systematik ihrer Orientierungen ist aus den wenigen Fällen jedoch nicht zu erkennen.

\section{Hohe Startbreiten $\left(\lambda_{\mathrm{s}}=65 \ldots 80^{\circ}\right)$}

Im Parameterbereich der Parker-Instabilität mit $m=1$ sind bei der höchsten untersuchten Startbreite $\lambda_{\mathrm{s}}=80^{\circ}$ in den Längenverteilungen keine Häufungspunkte zu erkennen (Abb. 10.6). In darunterliegenden Breiten, im Bereich der Strömungsinstabilität, zeigen sich besonders bei kleineren Feldstärken markante Vorzugslängen und -intervalle, die sich aber mit wachsendem $B_{\mathrm{s}}$ rasch zurückbilden. Für $\lambda_{\mathrm{s}}=70^{\circ}$ und $75^{\circ}$ findet man deutliche Häufungspunkte, in denen innerhalb $9^{\circ}$ breiter Längenintervalle teilweise über $20 \%$ aller bei dieser Breite ausbrechenden Flussröhren konzentriert sind. Bei $65^{\circ}$ zeigen die Verteilungsfunktionen einen deutlichen Übergang von schwächer ausgeprägten Vorzugslängen (bei hohen Feldstärken) über eine sehr stark lokalisierte (bei mittleren) hin zu Vorzugsintervallen; dieser kontinuierliche Verlauf ist vergleichbar mit den in Abb. 10.4 von oben nach unten aufgeführten Fällen. In diesen höheren Breiten scheint die Orientierung von Vorzugslängen ohne übergeordneter Systematik zu sein. Für $75^{\circ}$ Startbreite wird zwar die Umgebung der Quadraturpunkte etwas bevorzugt, für $\lambda_{\mathrm{s}}=65^{\circ}$ und $70^{\circ}$ sind die Verteilungen aber wesentlich uneinheitlicher.

\section{Niedere Startbreiten $\left(\lambda=0 \ldots 30^{\circ}\right)$}

Die Längenverteilungen äquatorialer Startbreiten $\left(\lambda_{\mathrm{s}} \leq 10^{\circ}\right)$ zeigen kaum nennenswerte Häufungspunkte. Lediglich bei vom Äquator startenden Flussröhren geringer Feldstärke 


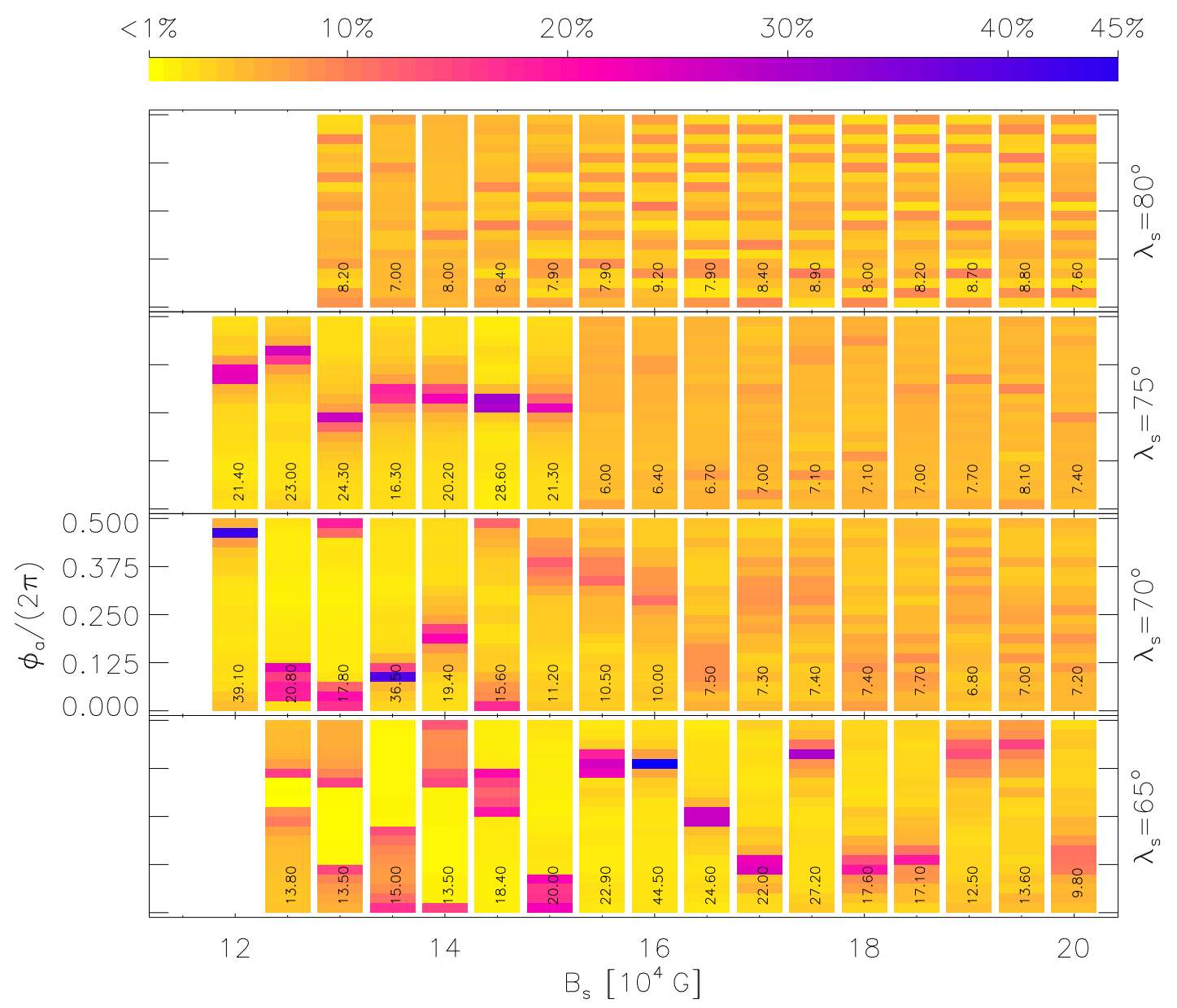

ABBILDUNG 10.6: Längenverteilungen ausbrechender Flussröhren bei hohen Startbreiten $\left(\lambda_{\mathrm{s}}=\right.$ $\left.65 \ldots 75^{\circ}\right)$. Lediglich bei $\lambda_{\mathrm{s}}=80^{\circ}$ ist die Ausbruchsursache die Parker-Instabilität mit Wellenzahl $m=1$, ansonsten die Strömungsinstabilität.

$\left(B_{\mathrm{s}} \simeq 10.5 \cdot 10^{4} \mathrm{G}\right)$ und solchen am oberen Ende des untersuchten Magnetfeldintervalls scheinen einige Längenbereiche hervorzutreten. Flussröhren mit Startbreiten zwischen $15 \ldots 25^{\circ}$ werden erst für Feldstärken $B_{\mathrm{s}} \gtrsim 15 \cdot 10^{4} \mathrm{G}$ instabil und beginnen ihren Aufstieg dann aufgrund der Parker-Instabilität mit der Wellenzahl $m=2$. In diesem speziellen Parameterbereich sind fast alle Ausbruchsverteilungen uneinheitlich.

\subsubsection{Zur Entstehung von Vorzugslängen und Sonderfällen}

Die Modifikation der Flussröhrenentwicklung durch den Begleitstern ist grundsätzlich ein kumulativer Effekt, der sich von der Overshoot-Region über die gesamte Konvektionszone bis zur Oberfläche erstreckt. Markante Vorzugslängen entstehen dabei fast ausschließlich bei Flussröhren die mit azimutaler Wellenzahl $m=2$ aufsteigen. Im Gegenzug zeigen Flussröhren der Wellenzahl $m=1$ meist keine Anzeichen einer inhomogenen Längenverteilung. Störungen der Wellenzahl $m=2$ reagieren wegen der Kongruenz zur 

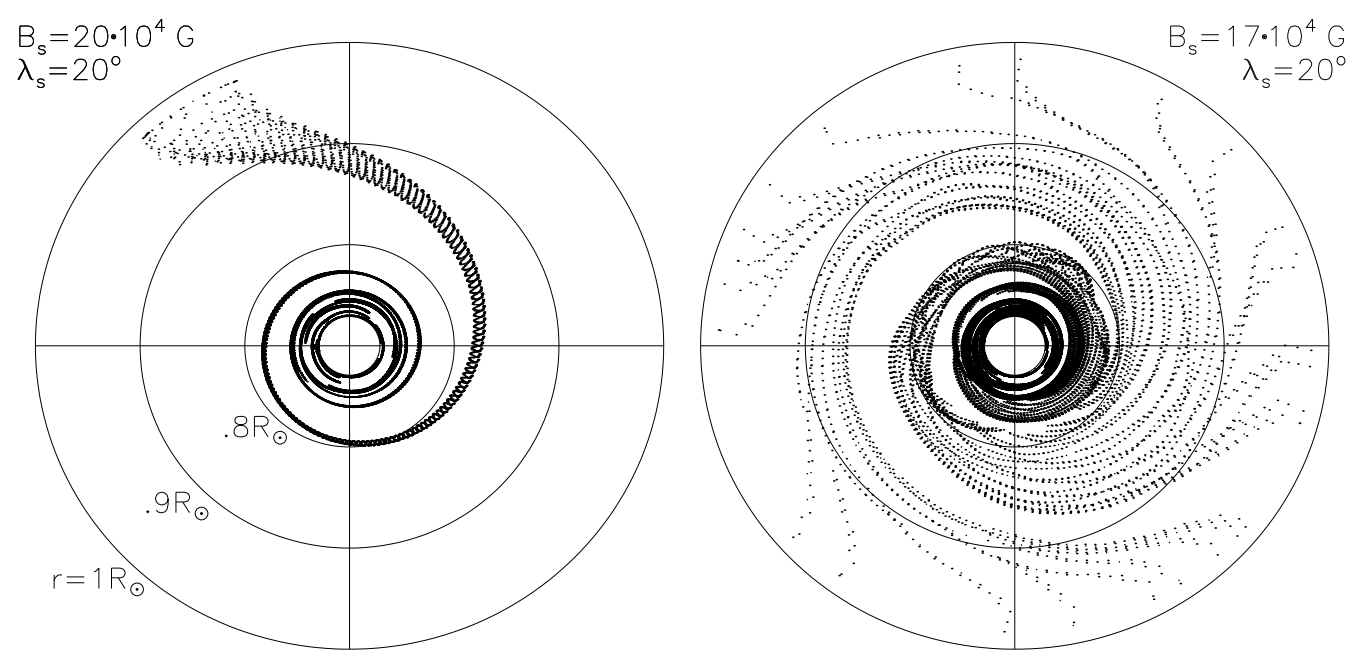

ABBILDUNG 10.7: Vergleich von Aufstiegstrajektorien in einem regulären Fall (links) und einem Sonderfall (rechts). In beiden Fällen wurde von den 25 berechneten Trajektorien jeweils die anfängliche Verschiebungen $\phi_{\mathrm{s}}$ abgezogen, sodass alle Kurven vom selben Punkt in der Overshoot-Region ausgehen. Das im regulären Fall kontinuierliche bzw. im Sonderfall irreguläre Auseinanderdriften der Trajektorien mit zunehmendem Radius $r$ geht auf den kumulativen Einfluss des Begleitsterns zurück.

azimutalen $\pi$-Periodizität der ursächlichen Variation des Doppelsternproblems besonders empfindlich und nachhaltig auf die Präsenz des Begleitsterns. Diese Resonanz ist vermutlich der Hauptgrund für das Auftreten der — im Verhältnis zum Einfluss der formalen Größenordnung $\mathcal{O}\left(\epsilon^{3}\right) \lesssim 10^{-3}-$ stark ausgeprägten Vorzugslängen.

Aus den Simulationen ergeben sich aber nicht immer kontinuierliche Funktionen $\phi_{\mathrm{a}}\left(\phi_{\mathrm{s}}\right)$ wie in Abb. 10.4. Für einige Ausgangskonfigurationen $\left(B_{\mathrm{s}}, \lambda_{\mathrm{s}}\right)$ existieren z.B. nur stückweise stetige Verläufe, die durch Sprünge voneinander getrennt sind, andere zeigen mehrmalige, willkürliche Wechsel zwischen verschiedenen Verläufen und wieder andere nur noch unsystematische Verteilungen, die keine Anzeichen der ursprünglich stetigen Verschiebungen $\Delta \phi_{\mathrm{s}}$ mehr erkennen lassen. Im Referenzfall beschränken sich diese Sonderfälle auf den Parameterbereich ${ }^{2} B_{\mathrm{s}} \gtrsim 15 \cdot 10^{4} \mathrm{G}$ und $\lambda_{\mathrm{s}} \simeq 15 \ldots 35^{\circ}$. Möglicherweise stellt deren atypisches Verhalten in diesem speziellen Bereich ein Analogon zur Resonanzkatastrophe eines oszillierenden Systems dar. In Abb. 10.7 wird die Entwicklung des jeweils höchsten Flussröhrenelements für einen repräsentativen Regelfall bzw. Sonderfall gegenübergestellt. Von den Trajektorien wurde jeweils die anfängliche Verschiebung $\phi_{\mathrm{s}}$ abgezogen, sodass sie alle am selben Punkt in der Overshoot-Region beginnen; das Auseinanderdriften der Verläufe ist ein Maß für den kumulativen Einfluss des Begleitsterns. Bei der Bildung regulärer Vorzugslängen wird die Flussröhrendynamik bei benachbarten Störungslängen $\phi_{\mathrm{s}}$ durch den Begleitstern nur soweit modifiziert, dass sich die zeitlichen Entwicklungen weitgehend ähneln und im Resultat eine stetige Modifikation der

\footnotetext{
${ }^{2}$ Der selbe Bereich zeichnet sich bei den Ausbruchszeiten $t_{\mathrm{a}}$ durch den verzögerten Aufstieg magnetischer Flussröhren aus. Es ist nicht klar ob dies eine Ursache, Folge oder Begleiterscheinung der Sonderfälle ist.
} 
Ausbruchslängen $\phi_{\mathrm{a}}$ ergeben. Überschreiten diese Modifikationen jedoch eine kritische Grenze, so kann die nicht-lineare Entwicklung schon durch sehr kleine Änderungen in völlig andere, irreguläre Bahnen gelenkt werden; kleine Änderung führen aufgrund des kumulativen Charakters schnell zu großen Abweichungen.

Aus dem $m=2$-Charakter der Instabilität folgen in der unteren Konvektionszone z.B. oft Phasensprünge des geometrisch höchsten Punkts der Flussröhre. Aus dem anfänglich toroidalen Flussring bilden sich an gegenüberliegenden Stellen zwei Schleifen, deren Auslenkung anfänglich von vergleichbarer Größenordnung ist und zwischen denen die Rolle der dominierenden Schleife solange hin und her wechselt, bis sich letztlich eine durchsetzt und an der Oberfläche ausbricht. Im Anfangsstadium dieser Entwicklung genügt bereits eine kleine Modifikation, wie sie etwa der Begleitstern bei Flussröhren mit verschiedenen Störungslängen hervorrufen kann, um durch einen zusätzlichen Wechsel der dominierenden Rolle einen Phasensprung in der Ausbruchsverteilung zu erzeugen.

\subsubsection{Oberflächenverteilung}

Im Gegensatz zu den Abschnitten 10.2.2 und 10.2.3, in denen Breiten- und Längenverteilungen jeweils separat betrachtet wurden, wird nun die resultierende Gesamtverteilung ausbrechender Flussröhren dargestellt. Die Abbn. 10.8 und 10.9 zeigen Oberflächenverteilungen in flächentreuen Polarprojektionen für die Feldstärkebereiche $B_{\mathrm{s}}=8 \ldots 13$. $10^{4} \mathrm{G}$ bzw. $14 \ldots 20 \cdot 10^{4} \mathrm{G}$, in denen Ausbruchsorte in einem dem Ausgangsraster in der Overshoot-Region entsprechenden Winkelraster von $9^{\circ} \times 5^{\circ}$ zusammengefasst sind. Aufgrund der polwärtigen Breitenablenkung und des längenabhängigen Einflusses des Begleitsterns weicht die Oberflächenverteilung von der gleichmäßigen Ausgangsverteilung am unteren Rand der Konvektionszone erheblich ab. Es existieren Gebiete, in denen sich Ausbrüche konzentrieren, und andere, die vollständig gemieden werden, wie beispielsweise der weitgehend ausbruchsfreie Gürtel unterhalb von etwa $25^{\circ}$ Breite.

Vorzugslängen und ihre Magnetfeldabhängigkeit sind in zweidimensionalen Oberflächenverteilungen weniger deutlich erkennbar als in den jeweiligen Längenverteilungen, da die Zahl der Ausbrüche nun über ein größeres Gebiet gemittelt wird. Durch die flächentreue Projektion wird dieser Effekt verstärkt, weil der systematische Eindruck in mittleren und hohen Breiten, der sich bei den eindimensionalen Verteilungen ergab, wegen der kleineren Oberflächenanteile gegenüber dem besonders bei größeren Feldstärken unsystematischen Verhalten in niederen Breiten zurücktritt. In dem aus physikalische Sicht relevanten Bereich kleiner Feldstärken ist das Gesamtbild übersichtlicher.

Die meisten der untersuchten Flussröhren geringer Feldstärke $\left(B_{\mathrm{s}} \lesssim 9 \cdot 10^{4} \mathrm{G}\right)$ sind stabil und brechen innerhalb des vorgegebenen Zeitrahmens nicht aus. Diejenigen, die instabil werden und aufsteigen, erreichen die Oberfläche bei mittleren Breiten. Ab etwa $10 \cdot 10^{4} \mathrm{G}$ kommen Ausbrüche bei niedrigeren und $\mathrm{ab} 11 \ldots 12 \cdot 10^{4} \mathrm{G}$ auch bei hohen und polaren Breiten hinzu. Es ist hauptsächlich auf die Strömungsinstabilität zurückzuführen, dass sowohl für hohe Feldstärken als auch bei hohen Breiten die Zahl ausbrechender Flussröhren relativ groß ist. Sie bewirkt bei ursprünglich als stabil eingestuften Ausgangskonfigurationen Ausbrüche auf vergleichsweise kurzen Zeitskalen. Vorzugslängen bei mittleren und hohen Breiten gehen auf Instabilitäten mit Wellenzahl $m=2$ zurück, wobei die Parker-Instabilität für die systematische Magnetfeldabhängigkeit verantwort- 

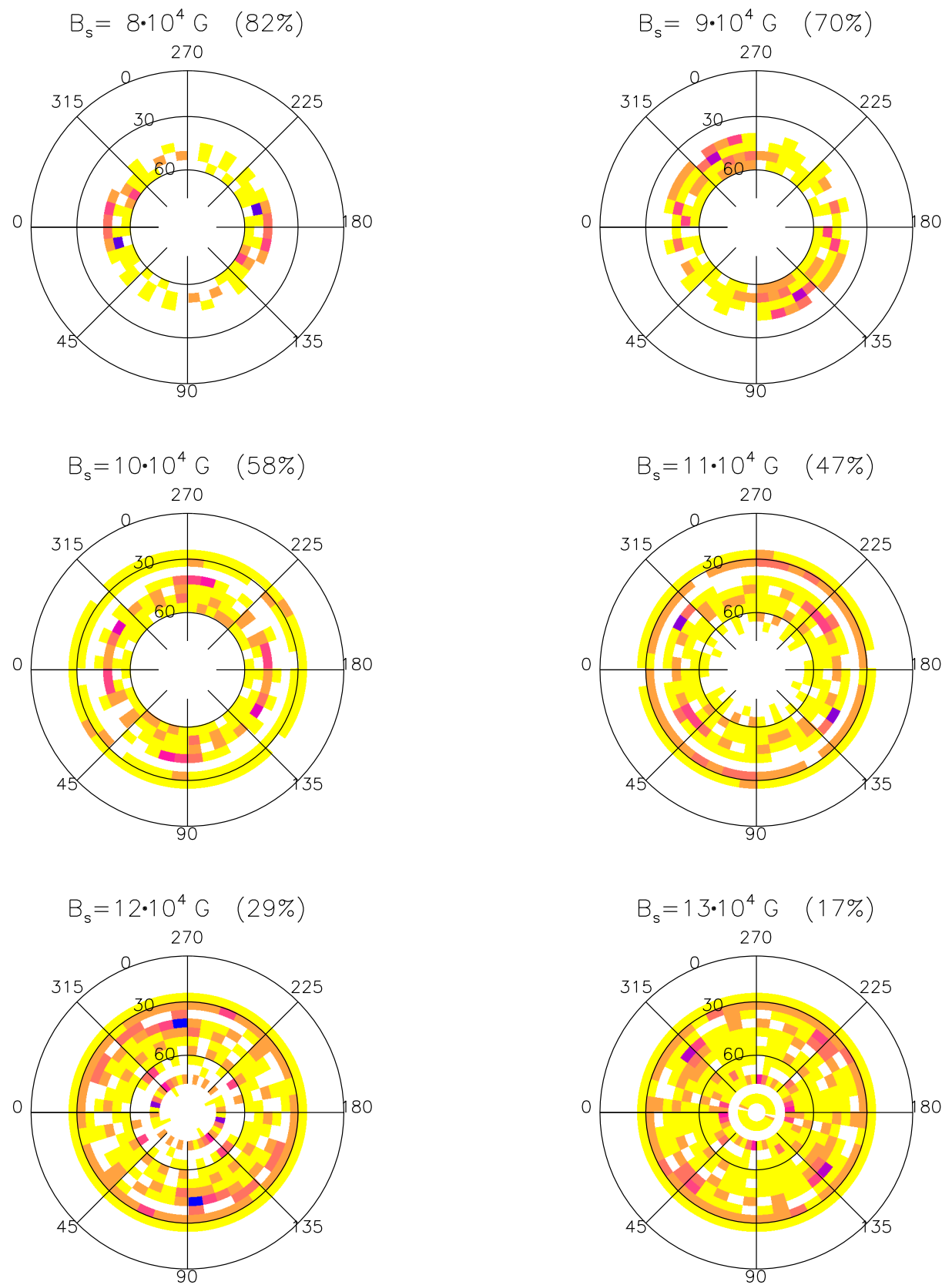

ABBILDUNG 10.8: Oberflächenverteilungen ausbrechender Flussröhren mit Ausgangsfeldstärken $B_{\mathrm{S}}=8 \ldots 13 \cdot 10^{4} \mathrm{G}$ (flächentreue Polarprojektion der nördlichen Hemisphäre). Der Begleitstern liegt in Richtung $0^{\circ}$ und die Quadraturpunkte $Q_{1,2}$ entsprechend bei $90^{\circ}$ und $270^{\circ}$. Die Rotation des Sterns erfolgt entgegen dem Uhrzeigersinn. Die Farbkodierung in Abb. 10.9 gibt die Anzahl ausbrechender Flussröhren innerhalb von Oberflächenarealen mit $9^{\circ}$ Längen- und $5^{\circ}$ Breitenausdehnung an und bezieht sich auf insgesamt 680 Flussröhren (340 individuelle Simulationen im Längenintervall $0 \ldots 180^{\circ}$ ). Der prozentuale Anteil der innerhalb eines Simulationszeitraums von $10^{9} \mathrm{~s}(\approx 32 \mathrm{yr})$ nicht ausbrechenden Flussröhren ist jeweils in Klammern angegeben. 

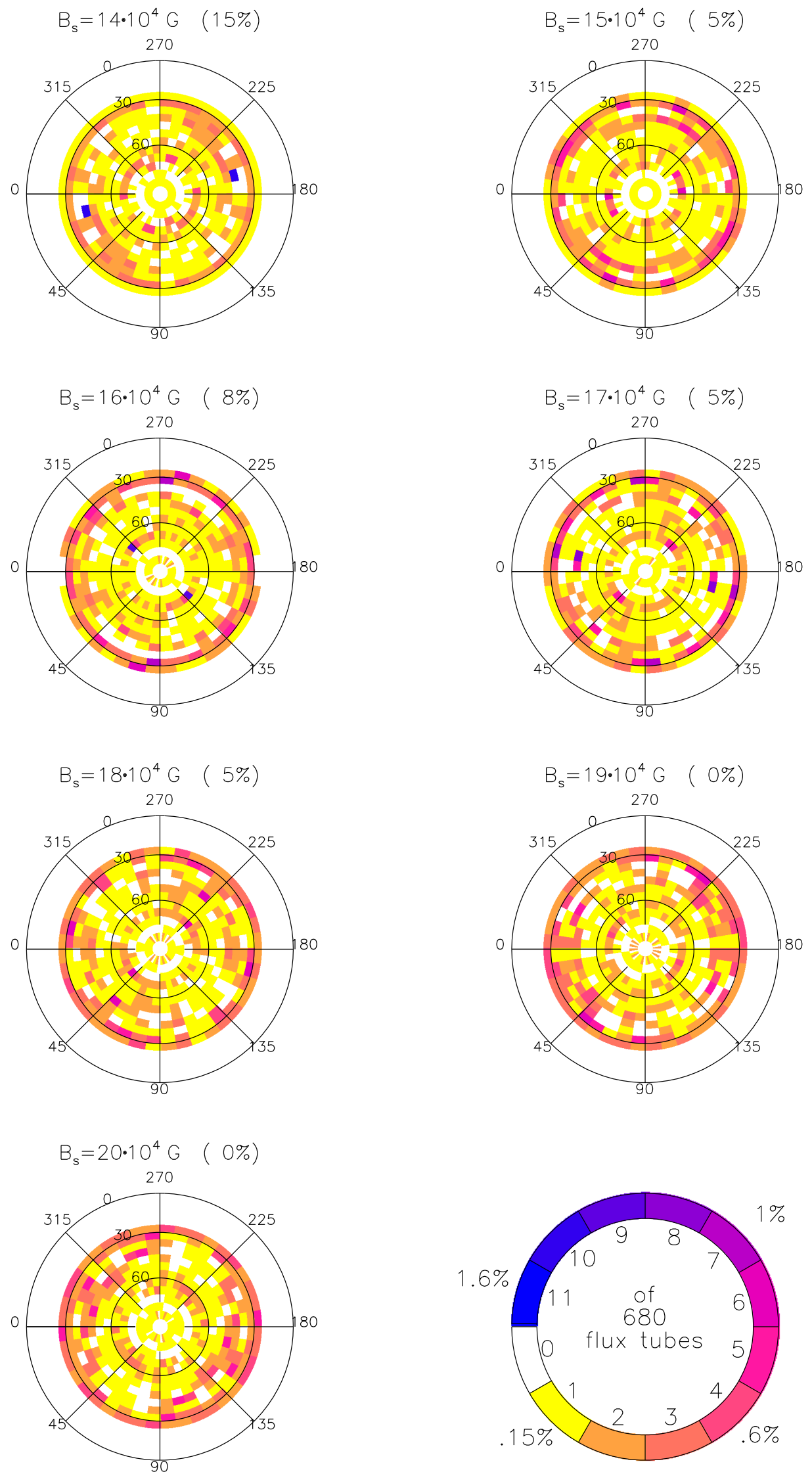

ABBILDUNG 10.9: Oberflächenverteilungen ausbrechender Flussröhren mit Ausgangsfeldstärken $B_{\mathrm{s}}=14 \ldots 20 \cdot 10^{4} \mathrm{G}$. 
lich ist. Die bei niedrigen Ausbruchsbreiten dominierende $m=1$-Instabilität erzeugt hingegen keine signifikanten Häufungen. Mit dem Einsetzen weitgehend unsystematischer Ausbrüche ab etwa $15 \cdot 10^{4} \mathrm{G}$ wird die Oberflächenverteilung unterhalb $40^{\circ}$ Breite uneinheitlich.

\subsection{Abhängigkeit von der Umlaufsperiode}

Um den Einfluss der Umlaufsdauer $T$ bzw. des Abstands $a$ des Begleitsterns auf die Ausprägung und Verteilung von Vorzugslängen zu untersuchen, wurden im Parameterbereich $\lambda_{\mathrm{s}}=0^{\circ} \ldots 80^{\circ}$ bzw. $B_{\mathrm{s}}=10 \ldots 15 \cdot 10^{4} \mathrm{G}$ vergleichbare Simulationen für $T=5 \mathrm{~d}$ und $1 \mathrm{~d}$ durchgeführt ${ }^{3}$; die entsprechenden Sternabstände sind $a \simeq 5.3 \mathrm{R}_{\odot}$ bzw. $15.5 \mathrm{R}_{\odot}$.

Im Fall $T=5 \mathrm{~d}$ zeigen die Längenverteilungen ausbrechender Flussröhren überwiegend keine signifikanten Vorzugslängen. Lediglich bei Startbreiten von $20^{\circ}, 30^{\circ}$ und $60^{\circ}$ sind an der Instabilitätsgrenze einige markante Häufungen zu finden. Vorzugslängen scheinen bei längeren Rotationsperioden zwar noch grundsätzlich möglich zu sein, im Vergleich mit dem selben Parameterbereich des Referenzfalls treten sie dann aber sehr viel sporadischer und ohne übergeordneter Systematik auf. Bei $T=2 \mathrm{~d}$ offenbaren Flussröhren mit Startbreiten unterhalb $30^{\circ}$ abermals einen unstetigen Verlauf der Ausbruchslängen $\phi_{\mathrm{a}}\left(\phi_{\mathrm{s}}\right)$, sodass ein direkter Vergleich zwischen allen drei Umlaufsperioden nur für mittlere Breiten möglich ist (Abb. 10.10). Die Ausprägung von Vorzugslängen und -intervallen nimmt mit abnehmender Umlaufsperiode erwartungsgemäß deutlich zu. Während bei langer Rotationsdauer praktisch keine Häufungen vorhanden sind, existieren im Referenzfall deutliche Vorzugslängen, die sich für kürzere Rotationsdauern noch stärker ausprägen. Dieser Übergang ist qualitativ mit den drei Fällen in Abb. 10.4 vergleichbar, der in diesem Fall dem wachsenden Einfluss des Begleitsterns mit abnehmendem Sternabstand $a$ zuzuordnen ist.

Ein Vergleich des Ausbruchsverhaltens bei hohen Startbreiten $\left(\lambda_{\mathrm{s}}=65 \ldots 75^{\circ}\right)$ gibt einen wichtigen Hinweis auf den Instabilitätstyp in diesem ursprünglich als quasi-stabil eingestuften Parameterbereich. Während bei $T=5 \mathrm{~d}$ alle betreffenden Flussröhren ausbrechen, erreicht bei $T=1 \mathrm{~d}$ keine von ihnen die Oberfläche. Im Referenzfall liegt die Instabilitätsgrenze hingegen bei $B_{\mathrm{s}} \simeq 12 \cdot 10^{4} \mathrm{G}$. Folglich verschiebt sie sich mit zunehmender Rotationsdauer zu kleineren Feldstärken. Dieses Verhalten steht aber im Gegensatz zu den Ergebnisse der Stabilitätsanalyse in Abschn. 9.4.3, nach denen sich die vom Begleitstern hervorgerufenen Instabilitätsplateau bei hohen Breiten für große Umlaufsperioden zurückbilden. Die Strömungsinstabilität hingegen liefert in diesem Fall eine Destabilisierung (vgl. Abschn. 13.2), d.h. einen qualitativen Verlauf, der konsistent mit den Simulationen ist. Ursprünglich als (quasi-)stabil eingestufte, aber dennoch ausbrechende Flussröhren verdanken ihren Aufstieg tatsächlich der Strömungsinstabilität und nicht den Doppelstern-bedingten Instabilitätsplateaus.

\footnotetext{
${ }^{3}$ Sternmasse $\left(M_{*}=1 \mathrm{M}_{\odot}\right)$ und Massenverhältnis $(q=1)$ wurden beibehalten
} 


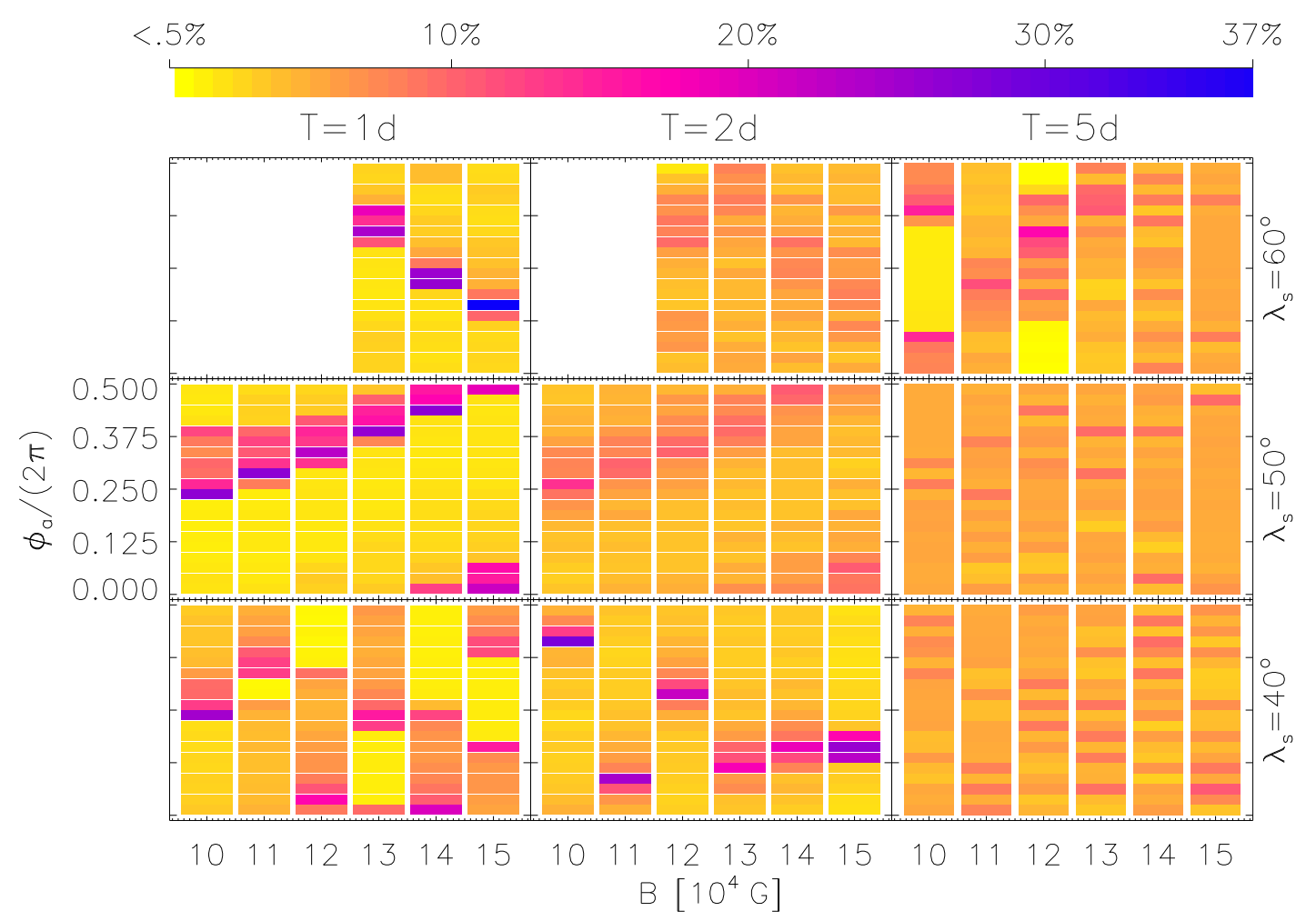

ABBILDUNG 10.10: Längenverteilungen von Flussröhren mit mittlerer Startbreiten bei Umlaufsdauern $T=1 \mathrm{~d}, 2 \mathrm{~d}$ und $5 \mathrm{~d}$ (von links nach rechts). Leere Felder markieren Konfigurationen bei denen innerhalb des Simulationzeitraums von $10^{9} \mathrm{~s}$ kein Ausbruch erfolgt.

\subsection{Diskussion}

Magnetische Flussröhren in der Konvektionszone von engen Doppelsternen werden während ihres Aufstiegs durch die Präsenz des Begleitsterns nachhaltig beeinflusst. Die Brechung der Axialsymmetrie stellt für ihre Entwicklung ein zusätzliches Charakteristikum dar, welches trotz seiner geringen Größe zu makroskopischen Effekten führt, die sich qualitativ von den bisher betrachteten deutlich unterscheiden: Durch die Gezeitenkraft und Deformation des Sternkörpers entstehen in Oberflächenverteilungen ausbrechender Flussröhren Inhomogenitäten in Form von lokalen Konzentrationen und bevorzugten bzw. gemiedenen Bereichen. Der Beobachtungsbefund, dass Fleckenherde oftmals an etwa $180^{\circ}$ auseinanderliegenden Stellen auf der Oberfläche lokalisiert sind, wird durch die dominierende $\pi$-Periodizität der Gezeitenwirkung sehr gut erklärt.

Der Gezeiteneinfluss auf ausbrechende Flussröhren hängt von der azimutalen Wellenzahl ihrer Entwicklung bzw. ursächlichen Instabilität ab. Dies zeigt sich besonders deutlich bei Flussröhren, die ihren Aufstieg aufgrund der Parker-Instabilität mit $m=2$ beginnen: Ihre dominierende Struktur mit zwei gegenüberliegenden, aufsteigenden Schleifen ermöglicht eine effektive, resonante Wechselwirkung mit dem kongruenten, $\pi$-periodischen Einfluss des Begleitsterns, der sich während des Aufsteigs von der 
Overshoot-Region bis zur Oberfläche aufsummiert. Sie zeigen markante Ausbruchskonzentrationen in z.T. sehr kleinen, bevorzugten Längenintervallen, deren Orientierung systematisch von der Anfangsbreite und -feldstärke abhängt. Offenbar gibt es keine feste Ausrichtung von Vorzugslängen bezüglich des Begleitsterns. Ihre - mit wachsender Feldstärke überwiegend im Rotationssinn des Sterns erfolgende - Verschiebung kann allerdings bei manchen Breiten so klein sein, dass diese Vorzugslängen relativ zum Begleiter nahezu fixiert sind. Im hier untersuchten Referenzfall sind die entsprechenden Häufungen (bei $\lambda_{\mathrm{s}} \sim 60^{\circ}$ ) zwar nur schwach ausgeprägt, aber da in diesem Breitenbereich Flussröhren unterschiedlicher Feldstärke offenbar die selbe Vorzugslänge haben, können sich bei dieser Stelle an der Oberfläche dennoch signifikante Fleckenherde bilden. Die Existenz von fixierten Vorzugslängen kann deshalb nicht grundsätzlich ausgeschlossen werden. Instabile Flussröhren mit der azimutalen Wellenzahl $m=1$, die überwiegend von niederen und sehr hohen Startbreiten aus aufsteigen, reagieren weitestgehend unempfindlich gegenüber der Gezeitenwirkung und tendieren kaum zur Bildung von Vorzugslängen. Im Gegensatz dazu stehen die nur schwer einzuordnenden Sonderfälle, deren irreguläres Ausbruchsverhalten sich nachhaltig auf die resultierenden Oberflächenverteilungen (vorzugsweise bei niederen Breiten) auswirkt, und deren Rolle nicht befriedigend geklärt ist. Ihre mit abnehmender Rotationsperiode wachsende Zahl legt die Annahme nahe, dass durch die zunehmende Gezeitenwirkung hier möglicherweise die Voraussetzung für eine kontinuierliche Abhängigkeit der Ausbruchsposition von der Anfangskonfiguration nicht mehr gegeben ist und die nicht-lineare Entwicklung chaotisch abläuft. Da sie keine übergeordnete Systematik erkennen lassen, ist anzunehmen, dass letztlich alle Ausbruchslängen gleichwahrscheinlich und etwaige Häufungspunkte darstellungsbedingt sind und im Grenzfall unendlich vieler Simulationen verschwinden. Eine systematische Abhängigkeit von der Ausgangskonfiguration konnte nur im Parameterbereich der Parker-Instabilität mit $m=2$ bei mittleren Breiten gefunden werden. In 'benachbarten' Parameterbereichen der Strömungsinstabilität zeigen ausbrechende Flussröhren zwar auch markante Inhomogenitäten, allerdings ohne übergeordnete Systematik. Dieses unterschiedliche Ausbruchsverhalten weist darauf hin, dass der ursächliche (auftriebs- oder strömungsbedingte) Instabilitätsmechanismus einen starken Einfluss auf die charakteristischen Eigenschaften von Vorzugslängen hat.

Die Simulationen bestätigen erwartungsgemäß den wachsenden Einfluss des Begleitsterns auf die Ausbruchsverteilung bei kürzeren Rotationsperioden, d.h. kleineren Sternabständen. Während für $T=5 \mathrm{~d}$ nur sporadische Ausbruchskonzentrationen auftreten, zeigen sie sich für $T=2 \mathrm{~d}$ bei einer überwiegenden Zahl von Ausgangskonfigurationen bereits systematisch und stark lokalisiert. Für kürzere Rotationsperioden gehen sie in Vorzugsintervalle über, was generell mit einer Aufweichung der Ausbruchskonzentration verbunden ist. Neben Fällen, in denen sich Ausbrüche über einen nur etwa $30^{\circ}$ breiten Bereich verteilen, zeigen die Simulationen für $T=1 \mathrm{~d}$ auch Vorzugsintervalle, die überwiegend durch die offensichtliche Meidung des verbleibenden Längenintervalls auffallen. Die Zahl der untersuchten Ausgangskonfigurationen ist zwar zu gering, um eine umfassende Aussage vorzunehmen, aber scheinbar gibt es einen Trend zum Rückgang stark lokalisierter Häufungen von Ausbruchsorten.

Der tatsächliche Ausbruchsort einer individuellen Flussröhre wird aufgrund sekundärer Einflüsse, wie beispielsweise der Wechselwirkung mit turbulenten Konvek- 
tionsbewegungen, der differentiellen Rotation, meridionalen Strömungen oder anderen Magnetfeldkonzentrationen von den simulierten Ausbruchsorten abweichen. Da aber die meisten - vielleicht sogar alle — dieser zusätzlichen Einflüsse in erster Näherung axialsymmetrische Modifikationen darstellen, werden sie bei einer großen Zahl ausbrechender Flussröhren nicht in der Lage sein den charakteristischen Doppelsterncharakter zu überdecken; in diesem Sinne machen die Resultate statistische Aussagen über eine hinreichend große Zahl vergleichbarer Flussröhren. Die ermittelten Oberflächenverteilungen basieren auf der Annahme einer gleichmäßigen Verteilung von Flussröhren (und ihrer Auslenkung aus der toroidalen Gleichgewichtslage) innerhalb der Overshoot-Region. Mit zusätzlichen Informationen über die zeitliche, räumliche und Feldstärken-Verteilung der Ausgangskonfigurationen könnten daraus konkrete Fleckenverteilungen konstruiert werden. Zieht man etwa, wie im Fall der Sonne, einen zyklischen Dynamomechanismus in Betracht, der eine Verstärkung magnetischer Felder am unteren Rand der Konvektionszone in periodisch variierenden Breiten hervorruft (Dynamowelle), so würde sich die resultierende Orientierung von Vorzugslängen an der Oberfläche aufgrund ihrer systematischen Abhängigkeit von der Ausgangskonfiguration $(B, \lambda)$ kontinuierlich verschieben und zu einer Migration führen, die bei Feldverstärkung überwiegend in Richtung der Sternrotation erfolgt. Üblicherweise wird dieses tatsächlich beobachtete Phänomen mit einer differentiellen Rotation des Sterns erklärt, die im vorliegenden Modell aber nicht enthalten ist. 


\section{Teil III}

\section{Strömungsinstabilität}





\section{Kapitel 11}

\section{Einleitung und Motivation}

Ein wichtiger Aspekt der idealen Magnetohydrodynamik ist das Konzept der 'eingefrorenen' Magnetfeldlinien: Entlang der Feldlinien kann sich das Plasma vom Magnetfeld unbeeinflusst bewegen, während es bei Bewegungen senkrecht dazu die Feldlinien mit sich zieht. Bei einer Bewegung der Flussröhre relativ zu ihrer Umgebung, mit der Geschwindigkeit $\mathbf{v}_{\perp}=\left(\mathbf{v}_{\mathrm{i}}-\mathbf{v}_{\mathrm{e}}\right)_{\perp}$, kann Plasma im Innern den Magnetfeldbereich nicht verlassen und externe Materie nicht darin eindringen; es setzt eine Umströmung der Röhre ein. Simulationen räumlich aufgelöster Flussröhren zeigen, wie diese durch die Wechselwirkung mit der Umgebung verformt werden und fragmentieren können (z.B. Schüssler 1979, Longcope et al. 1996), wenn nicht eine Transversalkomponente des Magnetfelds dem entgegenwirkt und die lokale Konzentration des longitudinalen Feldes näherungsweise erhält (Emonet \& Moreno-Insertis 1998, Fan et al. 1998, Abbett et al. 2000, 2001). Ein derartiges Verhalten kann im Rahmen der Näherung dünner Flussröhren jedoch nicht beschrieben werden ${ }^{1}$.

Für Röhrensegmente, deren Bogenlänge im Vergleich zu ihrem Krümmungsradius sehr klein ist, ähnelt die Situation der eines Zylinders, der in der Richtung senkrecht zu seiner Hauptachse umströmt wird. Aus der Hydrodynamik ist bekannt, dass die sich einstellende Strömung und deren Rückwirkung auf den Zylinder durch die dimensionslose Reynoldszahl

$$
R e=\frac{v_{\perp} d}{\nu}
$$

charakterisiert wird. Dabei ist $d$ der Durchmesser des Zylinders und $\nu$ die molekulare Viskosität des Mediums. Für $R e \ll 1$ ist das Strömungsprofil vor und hinter dem Zylinder symmetrisch; es liegt eine so genannte Kriechströmung vor. Bei etwas höheren Werten ( $R e \geq 1 \ldots 4$ ) bildet sich hinter dem Zylinder ein mit der Reynoldszahl anwachsender Totwasser-Bereich mit gegenläufigen Verwirbelungen. Bei turbulenten Strömungen mit Re $>10^{2} \ldots 10^{3}$ werden kontinuierlich Wirbel aus diesem Bereich abgelöst und davongetragen; es bildet sich die Kármánsche Wirbelstraße. Die Asymmetrie im Geschwindigkeitsprofil vor und hinter dem Zylinder erzeugt eine Druckdifferenz, die eine Widerstandskraft $\mathbf{f}_{D}$ auf den Zylinder ausübt. Diese Kraft hängt von den Strömungsverhält-

\footnotetext{
${ }^{1}$ Die Näherung geht davon aus, dass Flussröhren ihren zirkularen Querschnitt ständig beibehalten und der lokale Radius sich aus dem lateralen Druckgleichgewicht und der Erhaltung des magnetischen Flusses ergibt.
} 


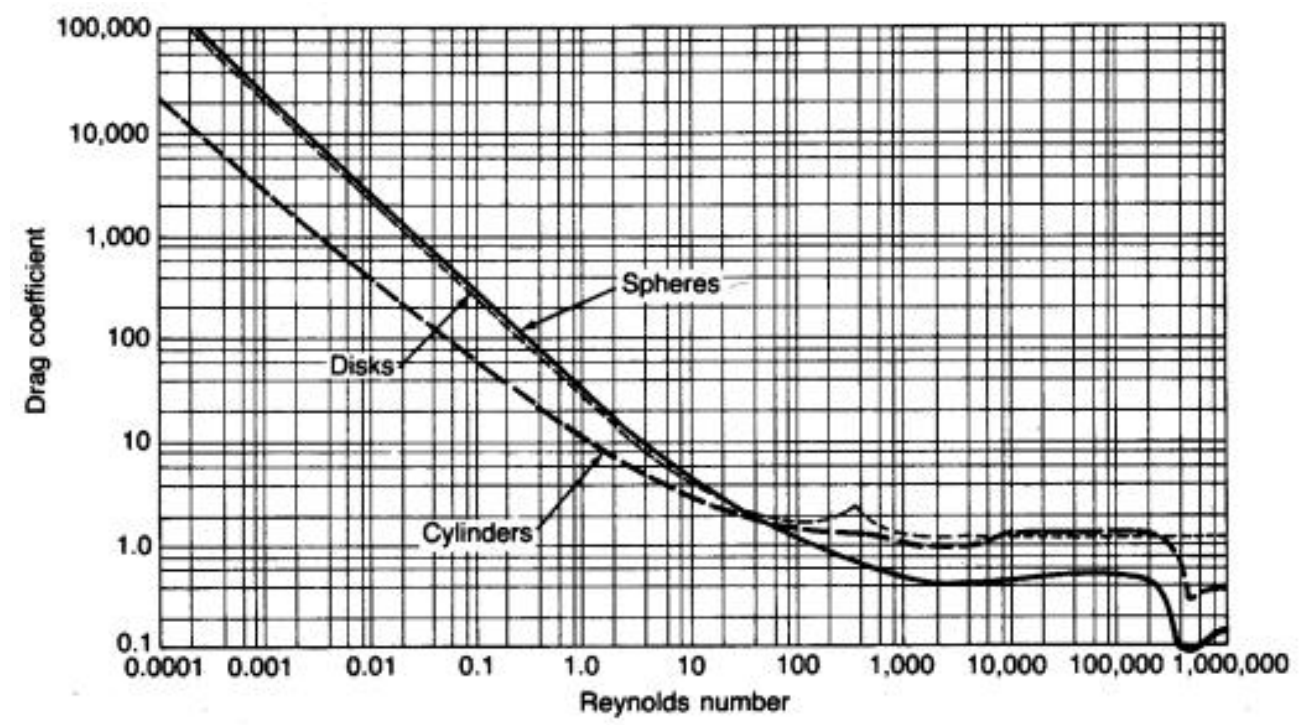

AbBILDUNG 11.1: Widerstandsbeiwert $C_{\mathrm{D}}$ als Funktion der Reynoldszahl (aus: Cooper \& Alley 1994). In dem Regime Stokesscher Reibung $R e \ll 1$ folgt für den Verlauf $C_{\mathrm{D}} \propto 1 / R e$. Oberhalb einer Übergangsregion ab $R e>10^{2} \ldots 10^{3}$ gilt für Zylinder und Kugel $C_{\mathrm{D}} \approx 1$.

nissen (laminar oder turbulent) in der dünnen Prandtlschen Grenzschicht ab und ist so orientiert das sie der Ursache ihrer Entstehung, der relativen Geschwindigkeitsdifferenz, entgegen wirkt: Es ist $\mathbf{f}_{\mathrm{D}} \propto-\hat{\mathbf{v}}_{\perp}$ mit $\left|\hat{\mathbf{v}}_{\perp}\right|=1$. Die Größe von $\mathbf{f}_{\mathrm{D}}$ wird üblicherweise durch den dimensionslosen Widerstandsbeiwert

$$
C_{\mathrm{D}}=\frac{\left|\mathbf{f}_{\mathrm{D}}\right| A}{\frac{1}{2} \rho v_{\perp}^{2} d}
$$

beschrieben, der nur von der Reynoldszahl abhängt und durch Messungen bei verschiedenen Strömungssituationen für das jeweilige Objekt empirisch bestimmt wird. Dabei ist $A$ die Querschnittsfläche und $d$ der Durchmesser des Zylinders. Abbildung 11.1 zeigt den typischen Verlauf von $C_{\mathrm{D}}(R e)$ für verschiedene geometrische Objekte. Im Bereich $10^{3}<R e<2 \ldots 3 \cdot 10^{5}$ ist $C_{\mathrm{D}} \simeq 1$ und die Widerstandskraft folgt dem Gesetz der Newtonschen (turbulenten) Reibung

$$
\mathbf{f}_{\mathrm{D}} \propto-v_{\perp}^{2} \hat{\mathbf{v}}_{\perp} .
$$

Im Bereich kleiner Reynoldszahlen $(R e \lesssim 1)$ befindet man sich hingegen im Regime Stokesscher Reibung und es gilt $C_{\mathrm{D}} \propto 1 / R e$, woraus folgt

$$
\mathbf{f}_{\mathrm{D}} \propto-v_{\perp} \hat{\mathbf{v}}_{\perp}
$$

Für die numerischen Simulationen im Rahmen dieser Arbeit wurde grundsätzlich davon ausgegangen, dass die Strömungsverhältnisse um aufsteigende magnetische Flussröhren in der Konvektionszone im Bereich großer Reynoldszahlen liegen und $\mathbf{f}_{\mathrm{D}}$ durch den aerodynamischen Strömungswiderstand (11.3) mit $C_{\mathrm{D}}=1$ adäquat beschrieben wird. Der 
Einfluss der in der Konvektionszone teilweise deutlich höheren turbulenten Viskosität auf den Strömungswiderstand wurde von Moreno-Insertis (1984) genauer untersucht. Die resultierenden Unterschiede zur obigen Annahme sind jedoch klein (Caligari 1995).

Aus der Verwendung von Gl. (11.3) ergibt sich, dass der Einfluss des Strömungswiderstands $\mathbf{f}_{\mathrm{D}}$ in der linearen Stabilitätsanalyse nicht berücksichtigt wird. Bei toroidalen Flussröhren im stationären Gleichgewicht tritt keine Relativbewegung der Flussröhre senkrecht zu ihrer Umgebung auf, d.h. im Gleichgewicht ist $\mathbf{v}_{\perp, 0}=0$. Aufgrund der quadratischen Abhängigkeit in $\mathbf{v}_{\perp}$ fällt bei der Linearisierung der Bewegungsgleichungen der Beitrag des Strömungswiderstands weg, wodurch eine Diskrepanz zwischen der linearen Analyse und den nicht-linearen Simulationen entsteht. Der Widerstandsterm ist ein Effekt zweiter Ordnung und sollte bei entsprechend kleinen Störungen der Flussröhre in den Simulationen nicht ins Gewicht fallen.

Im Laufe der Arbeit hat es sich allerdings erwiesen, dass unter bestimmten Umständen selbst eine geringe Reibung durchaus in der Lage ist eine neue Klasse von Instabilitäten zu bewirken, deren Anwachsraten mit denen der auftriebsbedingten Parker-Instabilität vergleichbar sind. Wie die lineare Stabilitätsanalyse magnetischer Flussringe in Doppelsternen zeigt (Abschn. 9.2), bilden sich mit abnehmender Rotationsperiode $T$ bei mittleren $\left(\lambda \sim 30 \ldots 45^{\circ}\right)$ und hohen Breiten $\left(\lambda \sim 65 \ldots 80^{\circ}\right)$ stabile bzw. quasi-stabile Bereiche (mit Anwachszeiten von mehreren tausend Tagen) aus, in denen Flussringe praktisch unanfällig gegenüber der Parker-Instabilität sind (vgl. Abb. 9.18 auf Seite 102). Die nichtlinearen Simulationen ergaben hingegen (vgl. Abb. 10.2), dass Flussröhren dieser Ausgangskonfigurationen nach kleinen Störungen ihrer Gleichgewichtslage dennoch instabil werden und mit Anwachszeiten aufsteigen, die mit denen der Parker-Instabilität vergleichbar sind. Bei höheren Feldstärken repräsentieren sie einen signifikanten Anteil aller an der Oberfläche ausbrechenden Flussröhren. Kontrollsimulationen ohne die Berücksichtigung der Reibung bestätigten die Ergebnisse der linearen Stabilitätsanalyse, wodurch der Strömungswiderstand $\mathbf{f}_{\mathrm{D}}$ als Ursache dieser neuartigen Instabilitäten identifiziert werden konnte.

Um diesen Zusammenhang nun genauer zu untersuchen, wird der Widerstandsterm (11.3) sowohl in der Stabilitätsanalyse als auch in den dazugehörigen Kontrollsimulationen vorübergehend durch den Stokesschen Reibungsterm

$$
\mathbf{f}_{\mathrm{St}}=-\rho C_{\mathrm{St}} \mathbf{v}_{\perp}
$$

ersetzt. Die Konstante $C_{\mathrm{St}}$ wird so gewählt, dass die Größenordnung der Reibung einer Flussröhre dem Wert des ursprünglichen Strömungswiderstands (11.3) entspricht; dabei wird von einer Flussröhre mit einem Durchmesser von $d=2000 \mathrm{~km}$ ausgegangen. Wir untersuchen zuerst den Einfluss der Reibung auf die Stabilität einer horizontalen Flussröhre, um den Mechanismus anhand eines einfachen Beispiels zu verdeutlichen und ihn dann auf toroidale Flussröhren in Einzelsternen anwenden zu können. 


\section{Kapitel 12}

\section{Horizontale Flussröhren in einer geschichteten Umgebung}

\subsection{Dispersionsgleichung und Instabilitätskriterium}

Untersucht wird eine horizontale, auftriebsneutrale magnetische Flussröhre in einer planparallel geschichteten Umgebung. Im Gleichgewichtszustand existiere zwischen der internen Strömung $\mathbf{v}_{\mathrm{i}, 0}$ und der externen Strömung $\mathbf{v}_{\mathrm{e}, 0}$ eine relative Geschwindigkeitsdifferenz $\mathbf{v}_{0}=\mathbf{v}_{\mathrm{i}, 0}-\mathbf{v}_{\mathrm{e}, 0}$ mit

$$
\left|\mathbf{v}_{0, \|}\right|=v \neq 0 \quad \text { und } \quad\left|\mathbf{v}_{0, \perp}\right|=0,
$$

wobei $\mathbf{v}_{0, \|}$ die tangentiale und $\mathbf{v}_{0, \perp}$ die (nicht vorhandene) normale Relativgeschwindigkeit der ungestörten Flussröhre bezeichnet. Durch die Auslenkung aus der Gleichgewichtslage kommt es aufgrund der (Stokesschen) Reibung zur Wechselwirkung zwischen den Röhrenelementen und der Strömung, die - in Abhängigkeit von der Phasengeschwindigkeit der resultierenden Welle - eine Abbremsung oder Beschleunigung entlang der aktuellen Normalenrichtung bewirkt:

$$
\mathbf{a}_{\mathrm{St}}=-C_{\mathrm{St}} \mathbf{v}_{\perp}
$$

Im Sinne der Näherung dünner Flussröhren (Abschn. 2.1.2) nehmen wir an, dass durch die Wechselwirkung in erster Näherung nur die Dynamik der Flussröhre, nicht aber die der externen Strömung beeinflusst wird.

Für die Stabilitätsanalyse dieses zweidimensionalen Problems wird der Formalismus des Einzelsternproblems im Grenzfall verschwindender Krümmung $(\kappa=0)$ und verschwindender Rotation $(\Omega=0)$ verwendet (vgl. Anhang A). Die Tangentialrichtung $\mathbf{t}_{0}$ zeige dabei entlang der ungestörten Flussröhre nach rechts, die Normalenrichtung $\mathbf{n}_{0}$ nach oben und die Gravitation nach unten: Es ist $\left(\mathbf{g} \cdot \mathbf{n}_{0}\right)=-g$. Die Auslenkung eines Röhrenelements wird durch den Vektor $\xi=\xi_{\mathrm{t}} \mathbf{t}_{0}+\xi_{\mathrm{n}} \mathbf{n}_{0}$ beschrieben. In dem Bezugssystem, in dem das Röhreninnere ruht $\left(\mathbf{v}_{\mathrm{i}, 0}=0\right)$ und die Umgebung sich mit der Relativgeschwindigkeit $\mathbf{v}_{\mathrm{e}, 0}=-v \mathbf{t}_{0}$ entlang der Flussröhre (nach links) bewegt, gilt für die Normalengeschwindigkeit der ausgelenkten Flussröhre

$$
\mathbf{v}_{\perp}=\left(\dot{\xi}_{\mathrm{n}}-v \xi_{\mathrm{n}}{ }^{\prime}\right) \mathbf{n}_{0}
$$


Der Punkt ' bezeichnet dabei die partielle Ableitung nach der Zeit $t$ und der Strich ' die nach der Bogenlänge $s$ der Flussröhre. Mit dem Exponentialansatz

$$
\xi(s, t) \propto \exp [i(k s+\omega t)]
$$

hat die entdimensionierte Dispersionsgleichung im Grenzfall $\beta \gg 1$ die vergleichsweise einfache Form

$$
\begin{aligned}
\tilde{v}^{4}- & i \tilde{C}_{\mathrm{St}} f \tilde{v}^{3}+\left(\frac{\Delta}{2} f^{2}-2+i \tilde{C}_{\mathrm{St}} f M_{\alpha}\right) \tilde{v}^{2} \\
& +i \tilde{C}_{\mathrm{St}} f \tilde{v}-\left[\left(\frac{\Delta}{2}+\frac{1}{\gamma^{2}}\right) f^{2}-1+i \tilde{C}_{\mathrm{St}} f M_{\alpha}\right]=0,
\end{aligned}
$$

wobei zur Entdimensionierung die Abkürzungen

$$
M_{\alpha}=\frac{v}{c_{a}} \quad, \quad f=\frac{1}{k H_{p}} \quad, \quad \tilde{v}=\left(\frac{\omega}{k}\right) \frac{1}{c_{a}} \quad \text { und } \quad \tilde{C}_{\mathrm{St}}=C_{\mathrm{St}} \frac{H_{p}}{c_{a}}
$$

verwendet wurden; dabei ist $k$ die Wellenzahl der Störung und $\omega$ die (komplexe) Eigenfrequenz. Die Alfvénsche Machzahl $M_{\alpha}$ dient als Maß für die externe Strömungsgeschwindigkeit und die Größe $f$ als Maß für die Wellenlänge der Störung. Die Phasengeschwindigkeit der Welle

$$
v_{\mathrm{ph}}=-\Re\left(\frac{\omega}{k}\right)
$$

wurde mit der Alfvéngeschwindigkeit $c_{\mathrm{a}}$ entdimensioniert; für $v_{\mathrm{ph}}>0$ breitet sich die Welle in positiver $\mathbf{t}_{0}$-Richtung aus. Der Imaginärteil $\Im(\tilde{v})$ ist proportional zur Anwachsrate eines Wellenmodus und beschreibt die Stabilitätseigenschaften der Flussröhre: Für $\Im(\tilde{v})>0$ klingen anfängliche Störungen mit der Zeit ab, während für $\Im(\tilde{v})<0$ eine anwachsende Instabilität vorliegt. Die Größen $H_{p}, \gamma$ und $\Delta$ charakterisieren die externe Schichtung. Abbildung 12.1 zeigt den Verlauf der entdimensionierten Anwachsrate $\left(\sim \Im\left(\tilde{v} / M_{\alpha}\right)\right)$ des instabilsten Wellenmodus als Funktion des Geschwindigkeitsverhältnisses $v_{\mathrm{ph}} / v$ für verschiedene Wellenlängen $f$. Bei kleinen Wellenlängen und langsamen Strömungsgeschwindigkeiten existieren gedämpfte Wellenmodi mit zeitlich abnehmender Amplitude. Bei (betragsmäßig) höheren Strömungsgeschwindigkeiten nimmt die Dämpfung ab und geht für

$$
\Re\left(\frac{\omega}{k}\right) \frac{1}{v}<1 \quad \Longleftrightarrow \quad v_{\mathrm{ph}}>v_{\mathrm{e}}=-v \quad \text { (externe Strömung) }
$$

in anwachsende Störungen der Flussröhre über. Störungen mit Wellenlängen $f \geq 2$ sind bei allen Strömungsgeschwindigkeiten instabil, wobei die Anwachsraten mit zunehmender Strömungsgeschwindigkeit $M_{\alpha}$ aber kontinuierlich abnehmen. Das Instabilitätskriterium (12.8) besagt, dass eine transversale Flussröhren-Welle, die aufgrund von Reibung mit einer schnelleren externen Strömung wechselwirkt instabil wird; mit anderen Worten: Die Amplitude einer transversalen Welle wird durch externen "Rückenwind" verstärkt ${ }^{1}$.

\footnotetext{
${ }^{1}$ Im Fall einer ungeschichteten, homogenen Umgebung entkoppeln die tangentiale und normale Komponente der Bewegungsgleichung. Störungen entlang der Richtung der Normalen $\mathbf{n}_{0}$ entsprechen rein transversalen Alfvénwellen mit der Ausbreitungsgeschwindigkeit $c_{a}$. In diesem Fall lässt sich zeigen, dass für das Einsetzen der reibungsbedingten Instabilität das Kriterium $v^{2}>v_{\mathrm{ph}}{ }^{2}=c_{a}^{2}$ erfüllt sein muss.
} 


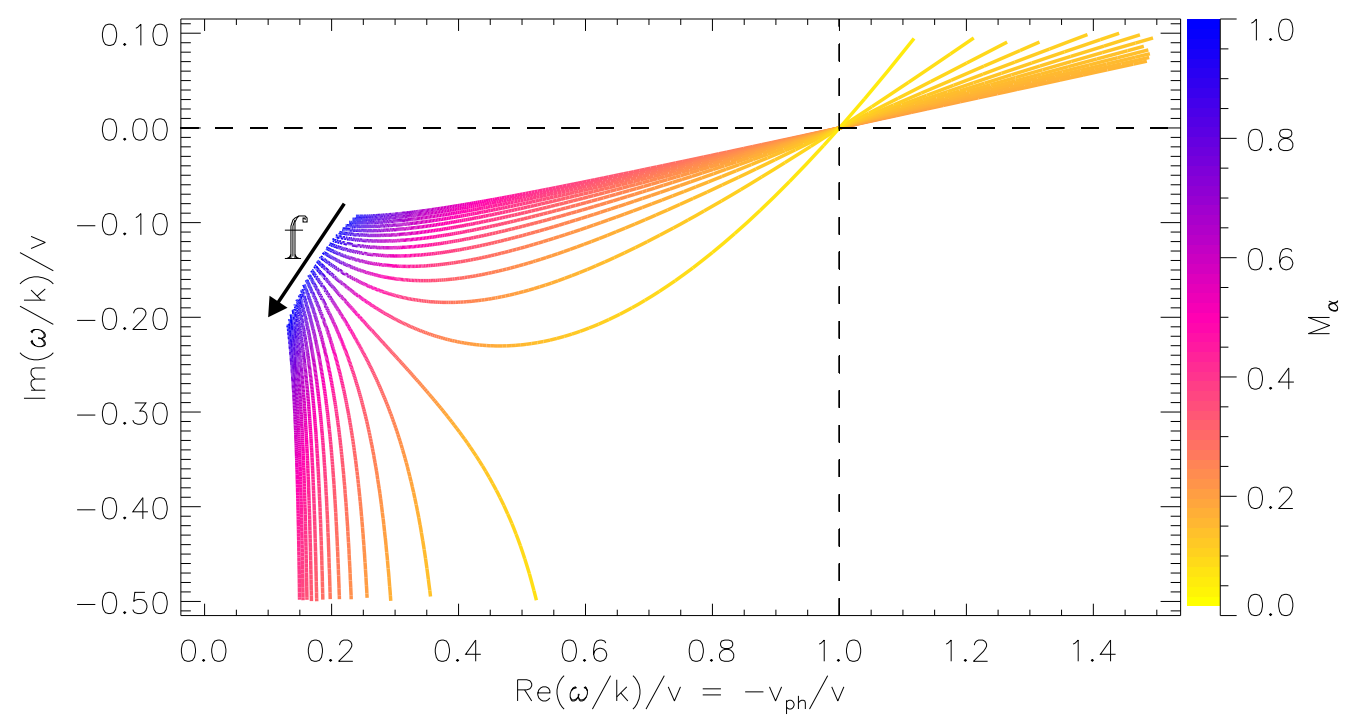

ABBILDUNG 12.1: Stabilitätsverhalten horizontaler Flussröhren bei Stokesscher Reibung für externe Strömungsgeschwindigkeiten $v=0 \ldots c_{a}$ und Wellenlängen $f=1.9 \ldots 2.1$. Alle anwachsenden Instabilitäten mit $\Im(\omega)<0$ liegen in dem Bereich $-v_{\mathrm{ph}} / v<1$. Es ist $\tilde{C}_{\mathrm{St}}=0.075, \Delta=$ -.22 und $\gamma=5 / 3$.

Da dieser Vorgang die Charakteristik einer Überstabilität hat, wächst die Auslenkung eines Röhrenelements nicht monoton, sondern oszillatorisch an. In der Bedingung (12.8) taucht die Reibungskonstante $C_{\mathrm{St}}$ nicht auf, d.h. das Instabilitätskriterium ist unabhängig von der Stärke der Reibung. Selbst eine kleine Reibung ist somit in der Lage, ein Anwachsen der Wellenamplitude zu bewirken; die Anwachsraten sind dann aber entsprechend klein.

In dem Bezugssystem, in dem die Umgebung ruht und das Innere der Flussröhre in Bewegung ist, hat das Instabilitätskriterium (12.8) die Form

$$
\Re\left(\frac{\omega}{k}\right) \frac{1}{v}<0 \quad \Longleftrightarrow \quad v_{\mathrm{ph}}>0 \quad \text { (interne Strömung). }
$$

Eine Flussröhre mit einer internen Strömung $\mathbf{v}_{\mathrm{i}}=v \mathbf{t}_{0}$ wird unter dem Einfluss Stokesscher Reibung instabil wenn sich eine Welle in die gleiche Richtung bewegt wie die Strömung selbst.

\subsection{Geometrisch-physikalische Deutung}

Um den Mechanismus der reibungsbedingten Instabilität zu verdeutlichen, beschreiben wir die Geschwindigkeits- und Kräfteverhältnisse im Ruhesystem der Flussröhre, d.h. im Gleichgewicht ist die interne Geschwindigkeit $v_{\mathrm{i}, 0}=0$ und die externe $v_{\mathrm{e}, 0}<0$. Wir betrachten einen Teil der ausgelenkten Flussröhre (Abb. 12.2), deren Phasengeschwindigkeit $v_{\text {ph }}$ negativ sei, d.h. die Welle bewege sich nach links. In diesem Fall erfährt ein Massenelement der Röhre in der vorausgehenden Flanke eines Wellenbergs eine Aufwärtsbewegung mit einer nach oben weisenden Geschwindigkeit $\mathbf{v}_{\mathrm{i}}$. Durch die Auslenkung 


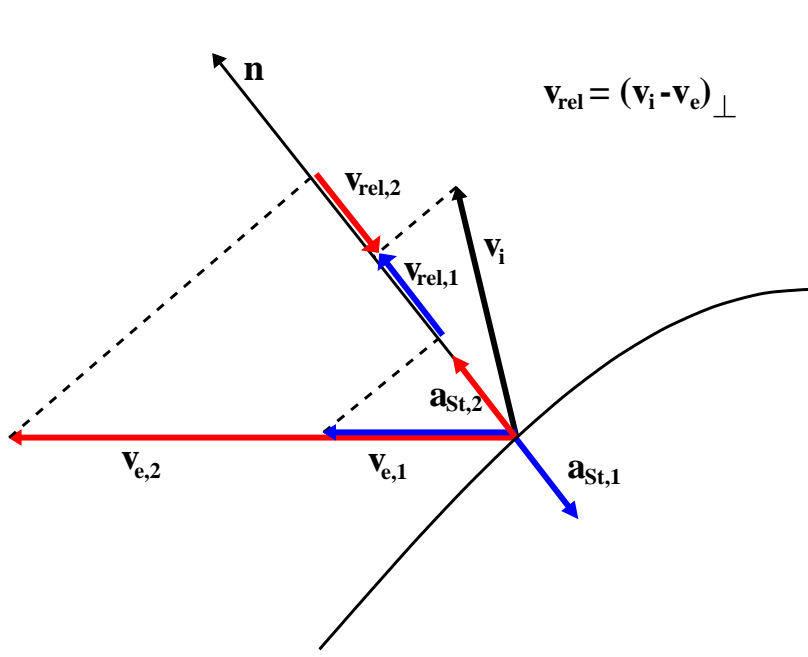

AbBILDUNG 12.2: Geschwindigkeits- und Kräfteverhältnisse bei einer Flussröhre mit Reibung in einer externen Strömung. Die Reibungskraft bewirkt, in Abhängigkeit von dem Verhältnis Strömungs- zu Phasengeschwindigkeit $\left(v_{\mathrm{e}}\right.$ bzw. $v_{\mathrm{ph}}$ ), eine Beschleunigung $\mathbf{a}_{\mathrm{St}}$, die der Geschwindigkeit des Röhrenelements $\mathbf{V}_{\mathrm{i}}$ entweder (1) entgegengerichtet $\left(\left|v_{\mathrm{e}}\right|<\left|v_{\mathrm{ph}}\right|\right.$, Dämpfung der Wellenamplitude) oder (2) gleichgerichtet $\left(\left|v_{\mathrm{e}}\right|>\left|v_{\mathrm{ph}}\right|\right)$, anwachsende Instabilität) ist.

entsteht eine Neigung der Flussröhre relativ zur externen Strömung, wodurch sich eine Angriffsfläche bildet, die vom umgebenden Medium umströmt werden muss. Die Reibungskraft ist der Relativgeschwindigkeit entlang der Normalenrichtung entgegengerichtet. Wie Abb. $12.2 \mathrm{zu}$ entnehmen ist, wird die Richtung (bzw. das Vorzeichen) der Relativgeschwindigkeit $\mathbf{v}_{\text {rel }}$ durch die Größe der externen Strömung $\mathbf{v}_{\mathrm{e}}$ bestimmt. Bei kleinen externen Strömungsgeschwindigkeiten $\left(\right.$ Fall $\left._{\mathrm{e}, 1}\right)$ bewirkt die Reibung eine der Röhrengeschwindigkeit $\mathbf{v}_{\mathrm{i}}$ entgegengerichtete Kraft, wodurch die weitere Auslenkung — zusätzlich zur magnetischen Rückstellkraft — gehemmt wird. Ist die externe Strömung größer als eine kritische Geschwindigkeit (Fall $\mathbf{v}_{\mathrm{e}, 2}$ ), so unterstützt die resultierende Reibungskraft die Auslenkung und bewirkt dadurch das Anwachsen der Amplitude. Dies zeigt sich auch bei der entsprechenden Änderung der kinetischen Energie des internen Massenelements aufgrund der Reibung:

$$
\frac{d E_{\mathrm{kin}}}{d t} \sim \mathbf{a}_{\mathrm{St}} \cdot \mathbf{v}_{\mathrm{i}}
$$

Im Fall $\mathbf{v}_{\mathrm{e}, 1}$ ist die Reibungskraft der internen Geschwindigkeit entgegengerichtet, $\mathbf{a}_{\mathrm{St}, 1}$. $\mathbf{v}_{\mathrm{i}}<0$, d.h. das Element verliert kinetische Energie und die Welle ist gedämpft. Im Fall $\mathbf{v}_{\mathrm{e}, 2}$ hingegen wird durch den Zuwachs an kinetischer Energie ein Anwachsen der Wellenamplitude bewirkt. Durch eine analoge Betrachtung ergibt sich, dass eine Welle, die in die entgegengesetzte Richtung (hier nach rechts) läuft immer gedämpft wird. Die Instabilität bezieht die benötigte Energie demzufolge aus der kinetischen Energie der Strömung. Dieses Resultat steht nicht in Widerspruch zu der Annahme, dass die Flussröhre die Strömung nicht beeinflusst. Die Situation ist vielmehr vergleichbar mit der in der Wärmelehre üblichen Annahme eines Wärmebads konstanter Temperatur, welches zwar in Kontakt mit einem thermodynamischen System steht, von diesem aber nicht beeinflusst wird. Im vorliegenden Fall ist im Rahmen der Näherung dünner Flussröhren die kinetische Energie der Umgebung erheblich größer als die im Röhreninneren und wird durch den Energietransfer daher praktisch kaum verändert.

Diese Instabilität zeigt, wie durch einen dissipativen Effekt wie Reibung Energie in Abhängigkeit von der relativen Strömungsgeschwindigkeit in beide Richtungen des Systems Flussröhre - Umgebung übertragen werden kann. 


\section{Kapitel 13}

\section{Toroidale Flussröhren in Einzelsternen}

Die in Kap. 12 gewonnenen Erkenntnisse über das Einsetzen der Strömungsinstabilität werden nun auf toroidale Flussröhren in Einzelsternen angewandt. Um diesen Fall untersuchen zu können, wird der Strömungswiderstand wieder durch den linearen Term

$$
\mathbf{a}_{\mathrm{St}}=-C_{\mathrm{St}} \mathbf{v}_{\perp}
$$

ersetzt, wobei $\mathbf{v}_{\perp}=\left(\mathbf{v}_{\mathrm{i}}-\mathbf{v}_{\mathrm{e}}\right)_{\perp}$ ist. Die Konstante $C_{\mathrm{St}}$ wird durch Vergleichsrechnungen zwischen den beiden Varianten (11.3) und (11.4) empirisch bestimmt und liegt hier bei $C_{\mathrm{St}} \simeq 10^{-7} \ldots 10^{-6}$.

\subsection{Schnelle Sternrotation}

Die Diagramme in Abb. 13.1 zeigen den Einfluss Stokesscher Reibung auf die Stabilität von Flussröhren in einem schnell rotierenden Einzelstern mit $T=2 \mathrm{~d}$. Alle Flussröhren, deren Magnetfeldstärke eine kritische Grenze überschreitet (hier $B_{\text {crit }} \simeq 4.5 \cdot 10^{4} \mathrm{G}$ ) sind instabil. Eine Ausnahme bilden lediglich extrem polnahe Breiten. Ausgehend von dieser Grenze nimmt die Anwachszeit $\tau$ zu höheren Feldstärken hin stark ab. Für Flussröhren hoher Magnetfeldstärke und mit Gleichgewichslagen in mittleren und hohen Breiten treten Strömungsinstabilitäten mit charakteristischen Anwachszeiten von einigen hundert Tagen und weniger auf, d.h. mit Zeitskalen, wie sie auch für die auftriebsbedingten ParkerInstabilitäten üblich sind. Eine Reihe numerischer Simulationen unter Verwendung des Stokesschen Reibungsansatzes bestätigen die Ergebnisse der linearen Analyse. Die Abbildungen 13.2 zeigen einen Vergleich zwischen der linearen Theorie und den numerischen Simulationen. Berechnet wurden die Anwachszeiten $\tau$ für Flussröhren bei konstantem Magnetfeld $\left(B=2 \cdot 10^{5} \mathrm{G}\right)$ bzw. konstanter Ausgangsbreite $\left(\lambda=35^{\circ}\right)$. Unter Berücksichtigung der unsicheren Bestimmung numerischer Anwachszeiten bedeutet die gute Übereinstimmung eine Bestätigung der linearen Ergebnisse. Das Instabilitätskriterium ${ }^{1}$ (12.9) wird darüber hinaus fast überall erfüllt. In dem in Abb. 13.1 dargestellten Fall wird das Kriterium aber durch die Existenz der Parker-Instabilitäten beeinflusst, sodass bei sehr hohen Breiten auch für $v_{\mathrm{ph}} \lesssim 0$ die Strömungsinstabilität einsetzt. Betrachtet man

\footnotetext{
${ }^{1}$ Die Simulationen erfolgen im Ruhesystem (mitrotierenden Bezugssystem) des Sterns, d.h. $v_{\mathrm{i}}=$ $v, v_{\mathrm{e}}=0$
} 


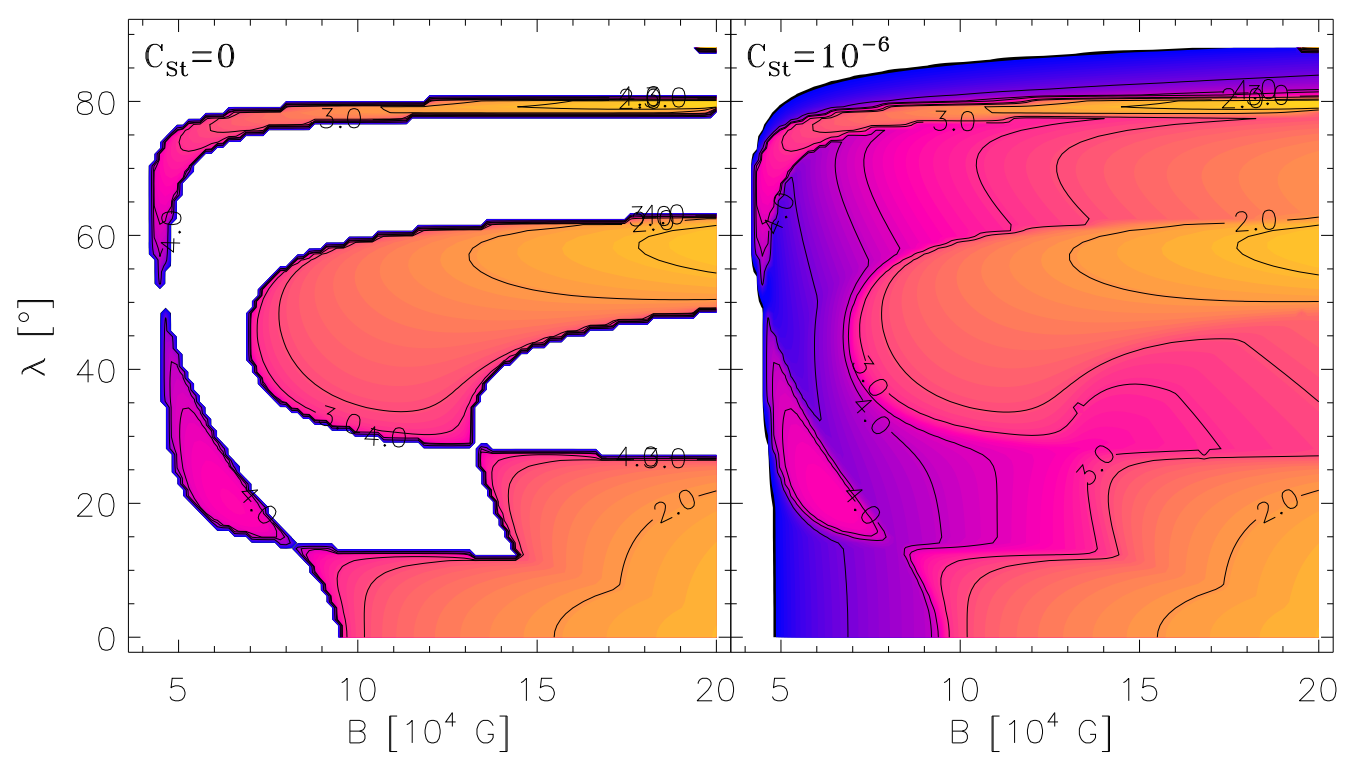

ABBILDUNG 13.1: Stabilitätsdiagramme für toroidale Flussröhren in einem Einzelstern mit $T=$ $2 \mathrm{~d}$ ohne (links) und mit Stokesscher Reibung (rechts). Ab einer Grenzfeldstärke (hier $B \gtrsim 4$. $10^{4} \mathrm{G}$ ) tritt die Strömungsinstabilität auf, deren Anwachsrate mit wachsendem $B$ größer wird. Die $\left(\log _{10} \tau\right)$-Niveaulinien markieren Anwachszeiten $\tau=10,50,100,500,1000,5000,10000$ und $50000 \mathrm{~d}$, die Darstellungsgrenze entspricht $\tau=10^{6} \mathrm{~d}$ und ist praktisch mit der Instabilitätsgrenze identisch.

allerdings Wellenmodi mit Wellenzahlen $m \geq 3$ (bei denen keine Parker-Instabilitäten auftreten), dann ist das Kriterium (12.9) sehr gut erfüllt.

Eine zusätzliche Parameterstudie zeigt, dass die Anwachsrate der Strömungsinstabilität mit wachsender Rotationsperiode zunimmt: Der Effekt ist (bei gleicher Magnetfeldstärke und Lage der Flussröhre sowie gleichem $C_{\mathrm{St}}$ ) bei schnell rotierenden Sternen kleiner als bei langsam rotierenden.

\subsection{Physikalische Interpretation}

Die Änderung der Anwachsrate lässt sich qualitativ auf der Grundlage der in Kap. 12 beschriebenen Strömungsinstabilität im Zusammenspiel mit der Gleichgewichtsbedingung toroidaler Flussröhren (Abschn. 2.2) verstehen. Die Gleichgewichtsbedingung (2.39) hat die Form

$$
M_{\alpha}=\frac{v}{c_{a}}=\frac{1}{\eta}\left(\sqrt{1+\eta^{2}}-1\right) \quad \text { mit } \quad \eta=c_{a} \frac{\kappa}{\Omega} \propto \frac{B T}{\cos \lambda} .
$$

Dabei ist $T$ die Rotationsperiode des Sterns und $\lambda$ die Gleichgewichts-Breite der Flussröhre. Die mit der Größe $\eta$ streng monoton wachsende magnetische Machzahl $M_{\alpha}$ ist in Abb. 2.2 dargestellt. Sie gibt an, wie groß die Strömung in der Flussröhre relativ zur Umgebung sein muss, damit die nach außen gerichtete Corioliskraft in der Lage ist, die nach innen gerichtete magnetische Krümmungskraft zu kompensieren. In Abschn. 12.1 

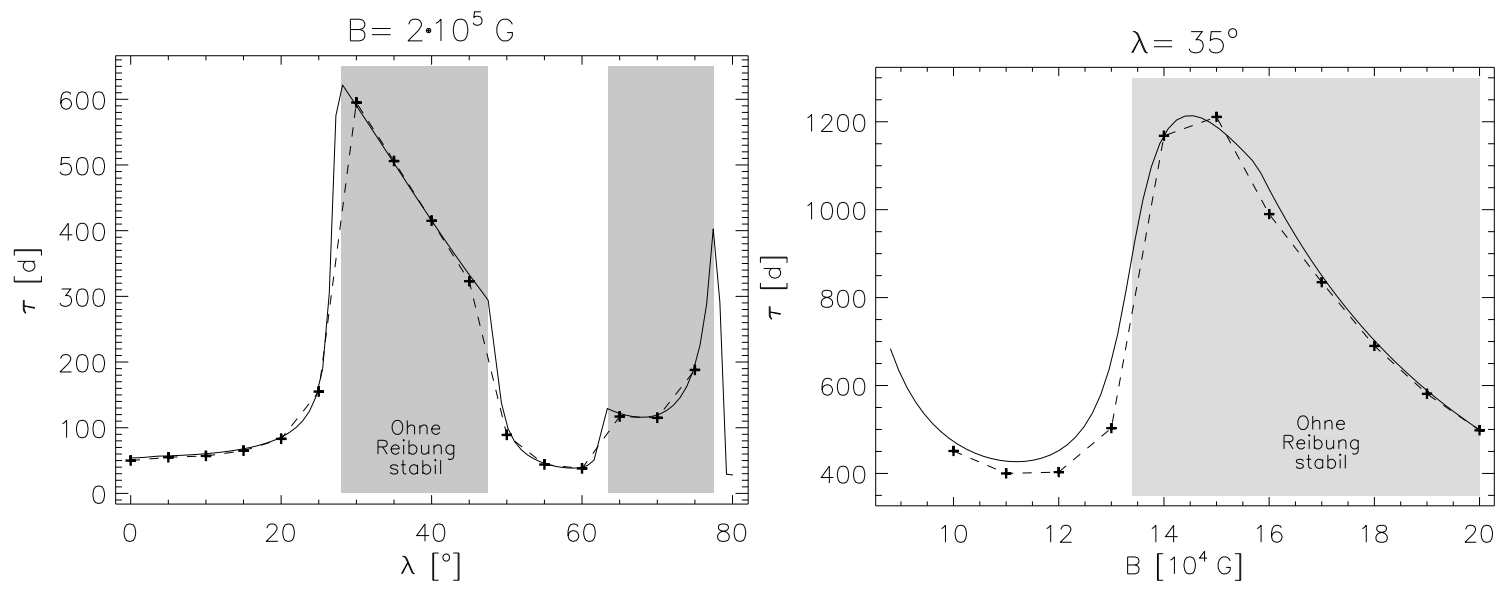

ABbILDUNG 13.2: Vergleich zwischen Stabilitätsanalyse (durchgezogene Linien) und Simulationen (gestrichelte Linien/Kreuze) bei Stokesscher Reibung mit $C_{\mathrm{St}}=10^{-6}$. Die Diagramme entsprechen Schnitte in Abb. 13.1 entlang der Breite $\lambda$ für $B=2 \cdot 10^{5} \mathrm{G}$ (links) und entlang $B$ für $\lambda=35^{\circ}$ (rechts).

wurde gezeigt, dass eine transversale Flussröhren-Welle instabil wird, wenn das Kriterium (12.8/12.9) erfüllt ist. Flussringe mit kleinen Feldstärken benötigen nur eine kleine Strömungsdifferenz $v$, um im stationären Gleichgewicht zu sein. Das Instabilitätskriterium ist für sie nicht erfüllt. Nimmt $B \mathrm{zu}(\eta \uparrow)$, so ist ein immer größeres $v$ notwendig, um ein Kräftegleichgewicht herzustellen. Bei einer kritischen Feldstärke $B_{\text {crit }}$ wird letztlich die Grenzgeschwindigkeit überschritten, ab der Instabilität eintritt. Bei höheren Breiten $\lambda$ ist der Krümmungsradius der Flussröhre geringer $(\eta \uparrow)$, wodurch wiederum die hohe Geschwindigkeit $v$ und das Einsetzen der Instabilität erklärt wird. Bei sehr hohen Breiten und entsprechend engen Flussringen macht sich dagegen die stabilisierende Wirkung der magnetischen Krümmungkraft bemerkbar, was zu einer Zunahme von $\tau$ führt. Darüber hinaus verdeutlicht Gl. (13.2), warum die Instabilität bei langsam rotierenden Sternen mit wachsendem $T(\eta \uparrow)$ stärker wird: Durch die Abnahme der Rotationsrate verliert die Corioliskraft an Effektivität. Um dies auszugleichen, ist eine größere Strömungsgeschwindigkeit $v$ notwendig, wodurch die Instabilität verstärkt wird. Bei dieser qualitativen Betrachtung ist grundsätzlich zu beachten, dass sich bei der Änderung der Gleichgewichtsbedinungen $(B, \lambda, T, \ldots)$ i.Allg. auch die Phasengeschwindigkeit $v_{\mathrm{ph}}$ des Wellenmodus ändert.

\subsection{Der Fall der Sonne}

Die Ausführungen im letzten Abschnitt zeigen, dass die Wirkung der Strömungsinstabilität mit wachsender Rotationsperiode zunimmt. Dies wirft die Frage nach dem Einfluss dieser Instabilität im Fall der Sonne mit $T \simeq 27 \mathrm{~d}$ auf. Frühere Arbeiten (van Ballegooijen 1982, Fan et al. 1993, Caligari et al. 1995) weisen darauf hin, dass zur Erklärung der beobachteten Charakteristika von Sonnenfleckengruppen magnetische Flussröhren mit SuperÄquipartitionsfeldstärken von der Größenordnung $B \sim 10^{5} \mathrm{G}$ in der Overshoot-Region 


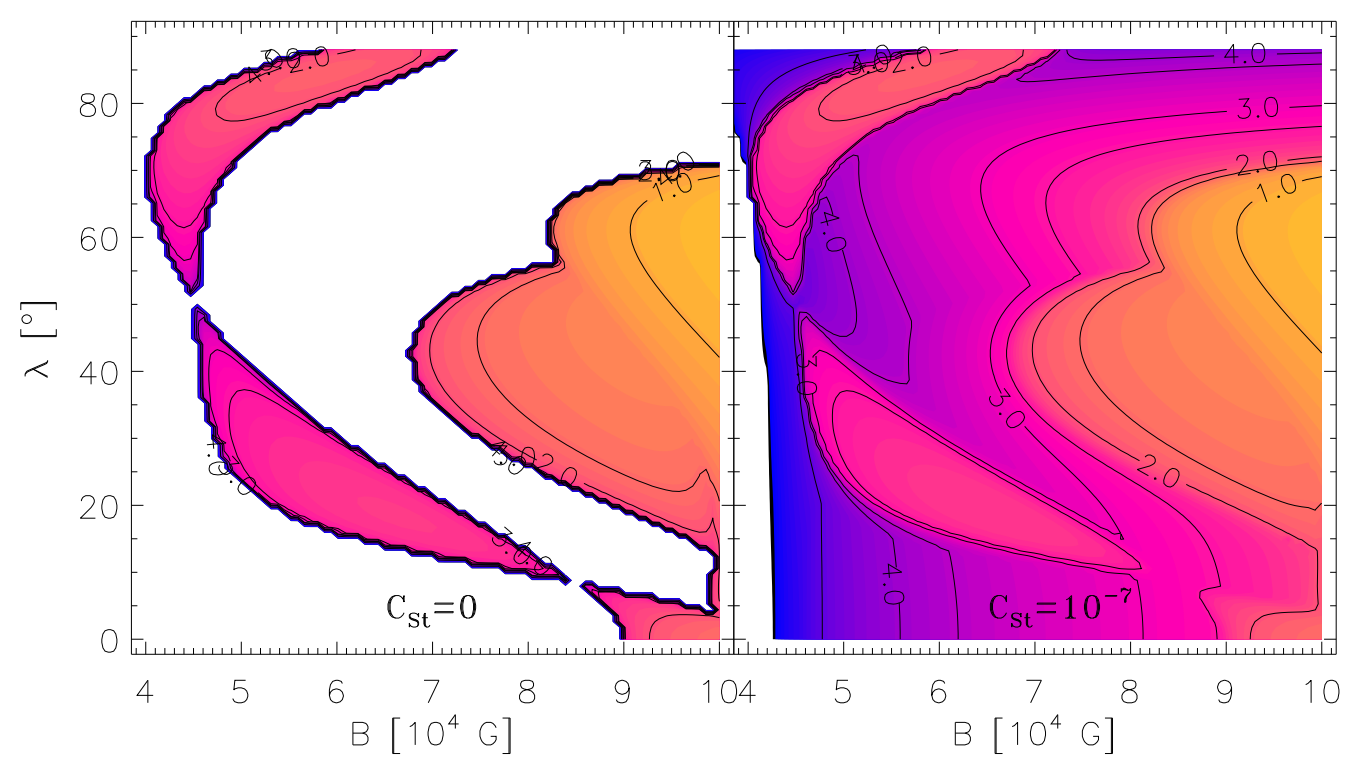

AbBILDUng 13.3: Gleiche Darstellung wie in Abb. 13.1 jedoch für einen Stern mit $T=27 \mathrm{~d}$. Durch die Reibung setzt bei kleinen Feldstärken die Strömungsinstabilität ein, die für alle höheren Feldstärken einen 'Instabilitätshintergrund' bildet. Im Vergleich zu schnell rotierenden Sternen ist dieser Effekt auch bei hohen Breiten wirksam, wenngleich mit vergleichsweise langen Anwachszeiten. Der Pol $\left(\lambda>88^{\circ}\right)$ wurde aus numerischen Gründen ausgegrenzt.

der Sonne angenommen werden müssen. Diese Forderung setzt voraus, dass Flussröhren geringerer Feldstärke lange genug am unteren Rand der Konvektionszone gespeichert werden, um dem Dynamo-Mechanismus ausreichend Zeit zur Feldverstärkung zu geben. Flussröhren geringerer Feldstärke sollten stabil sein oder zumindest Anwachszeiten unterliegen, die groß sind im Vergleich zu den Zeitskalen, auf denen der Dynamo operiert. Legt man für die obere Grenze dieser charakteristischen Zeitskalen einen halben Aktivitätszyklus zugrunde, so entspricht dies bei der Sonne etwa 11 Jahren und die Forderung nach Quasi-Stabilität demnach Anwachszeiten von $\tau \geq 4 \cdot 10^{3} \mathrm{~d}$. Abbildung 13.3 zeigt einen Vergleich von Stabilitätsdiagrammen für ein Sonnenmodell mit $\Omega=\Omega \simeq 2.7 \cdot 10^{-6} \mathrm{~s}^{-1}$ mit bzw. ohne Reibung. Die Reibungskonstante wurde durch einen Vergleich von Simulationen zu $C_{\mathrm{St}}=10^{-7}$ bestimmt. Die Grenze der Strömungsinstabilität liegt bei etwa $4.5 \cdot 10^{4} \mathrm{G}$. Anwachsraten von einigen tausend Tagen werden bei etwa $6 \ldots 7 \cdot 10^{4} \mathrm{G}$ erreicht. Berücksichtigt man, dass die angenommene Zeitskala von 4000 Tagen für den Dynamo-Mechanismus eine Obergrenze darstellt und der tatsächliche Wert vermutlich deutlich darunter liegt, so kann man davon ausgehen, dass die früheren Ergebnisse durch den zusätzlichen Aspekt der Strömungsinstabilität nicht grundlegend in Frage gestellt werden. 


\section{Kapitel 14}

\section{Diskussion}

Das Modell einer dünnen magnetischen Flussröhre in einer nicht-magnetischen Umgebung findet vielfältige Anwendung in verschiedenen Bereichen der Astrophysik. Dementsprechend umfangreich sind die Untersuchungen, die sich mit dem Gleichgewicht und der (In-)Stabilität verschiedener Magnetfeldkonfigurationen befassen. Grundlegende Darstellungen dieser Probleme finden sich z.B. bei Priest (1982) und Stix (1989). Für Flussröhren in der Konvektionszone und Atmosphäre der Sonne wird hier auf Spruit \& van Ballegooijen (1982), Ferriz-Mas \& Schüssler (1995), Schmitt (1998) und Pintér et al. (1999) verwiesen. Eine Untersuchung in Hinblick auf den Einfluss der Reibung erfolgte bisher nicht ${ }^{1}$.

Durch die Existenz einer senkrecht zur Flussröhre wirkenden Reibungskraft wird eine Strömungsinstabilität angeregt, wenn die Phasengeschwindigkeit eines Wellenmodus kleiner ist als die relative Strömungsgeschwindigkeit. Dieses Ergebnis beruht auf Untersuchungen der Stokesschen Reibungskraft. Deren Verwendung ist im Fall dünner Flussröhren in Sternen aus physikalischer Sicht zwar zweifelhaft, ermöglicht aber andererseits die analytische Behandlung. Wie sich zeigt, ist das Instabilitätskriterium (12.8/ 12.9) unabhängig von der Proportionalitätskonstante $C_{\mathrm{St}}$ der Stokesschen Reibung, sodass die Instabilität generell existieren sollte, wenn der funktionale Zusammenhang einer der Normalengeschwindigkeit entgegengesetzten Reibungskraft, d.h. $f_{D} \propto-\hat{\mathbf{v}}_{\perp}$, gegeben ist. Diese Annahme wird durch die Simulationen in Kap. 10 unter Verwendung des Strömungswiderstands (11.3) bestätigt.

Für die Stabilitätsbetrachtung wurde die Größe des Parameters $C_{\mathrm{St}}$ durch einen Vergleich mit Simulationen mit dem quadratischen Strömungswiderstand (11.3) bestimmt. Dieser Zusammenhang beinhaltet, dass der Strömungswiderstand für Flussröhren mit kleinem Querschnitts-Durchmesser $d$ effektiv größer ist als für solche mit einem großen Durchmesser; $d_{1}<d_{2}$ führt zu $C_{\mathrm{St}, \mathrm{d} 1}>C_{\mathrm{St}, \mathrm{d} 2}$. Bei gleicher Feldstärke $B$ unterliegen Flussröhren mit einem kleinen Querschnitt einer größeren reibungsbedingten Instabilität und sollten demnach mit kürzeren Anwachszeiten die Overshoot-Region verlassen. Die Konsequenz wäre, dass der magnetische Fluss bei hohen Feldstärken überwiegend in der Form von wenigen, vergleichsweise dicken Flussröhren vorliegt und nicht in vielen

\footnotetext{
${ }^{1}$ Die Instabilität wird nicht durch Druckvariationen angeregt, sondern basiert auf der dissipativen Wechselwirkung mit der Umgebung bei der Umströmung der Flussröhre; sie unterscheidet sich dadurch grundlegend von der Kelvin-Helmholtz-Instabilität (vgl. D’Silva \& Choudhuri 1991).
} 
dünnen.

Im Fall schneller Sternrotation (Kap. 10) tritt die Strömungsinstabilität mit vergleichsweise kurzen Anwachsraten nur bei hohen Feldstärken auf. Da bereits bei kleineren Feldstärken auftriebsbedingte Instabilitäten das Ausbrechen von magnetischem Fluss verursachen, ist der Beitrag der Strömungsinstabilität in diesem Fall nur von untergeordneter Bedeutung. Dennoch findet er in den Simulationen volle Berücksichtigung. 


\section{Anhang A}

\section{Koeffizientenmatrizen}

Wie in Abschn. 9.2.1 beschrieben, führt die Linearisierung der Bewegungsgleichungen (2.33) - (2.36) bei kleinen Störungen $\xi$ einer Flussröhre aus ihrer toroidalen Gleichgewichtslage zur Matrizengleichung (9.12). Dabei wurde der Exponentialansatz (9.10) und die Normierung (9.11) verwendet. Mit den konstanten Matrizen

$$
\mathcal{E}=\left(\begin{array}{lll}
1 & 0 & 0 \\
0 & 1 & 0 \\
0 & 0 & 1
\end{array}\right), \quad \mathcal{Y}=\left(\begin{array}{ccc}
0 & 1 & 0 \\
-1 & 0 & 0 \\
0 & 0 & 0
\end{array}\right) \text { und } \mathcal{Z}=\left(\begin{array}{ccc}
0 & 0 & 1 \\
0 & 0 & 0 \\
-1 & 0 & 0
\end{array}\right)
$$

haben die Koeffizientmatrizen im Grenzfall $\beta \gg 1$ folgende Form:

$$
\begin{aligned}
& \mathcal{M}_{\phi t}=-\mathcal{E} \\
& \mathcal{M}_{t t}=-\frac{1}{4} \frac{1-M_{\alpha}{ }^{2}}{M_{\alpha}{ }^{2}} \mathcal{E} \\
& \mathcal{M}_{\phi}=\left(\mathcal{F}_{1} \frac{g_{\mathrm{n}}}{g_{*}}-1\right) \mathcal{Y}+\mathcal{F}_{1} \frac{g_{\mathrm{b}}}{g_{*}} \mathcal{Z} \\
& \mathcal{M}_{t}=\frac{1}{2} \frac{1+M_{\alpha}{ }^{2}}{M_{\alpha}{ }^{2}} \mathcal{Y} \\
& \mathcal{M}_{\xi}=\mathcal{F}_{1} \frac{1}{g_{*}}\left(\begin{array}{ccc}
g_{\mathrm{t}}{ }^{\prime} & -g_{\mathrm{t}} & 0 \\
g_{\mathrm{t}} & 2 g_{\mathrm{n}} & g_{\mathrm{b}} \\
0 & g_{\mathrm{b}} & 0
\end{array}\right)+\mathcal{F}_{3} \frac{1}{g_{*}^{2}}\left(\begin{array}{ccc}
g_{\mathrm{t}} g_{\mathrm{t}} & g_{\mathrm{t}} g_{\mathrm{n}} & g_{\mathrm{t}} g_{\mathrm{b}} \\
g_{\mathrm{n}} g_{\mathrm{t}} & g_{\mathrm{n}} g_{\mathrm{n}} & g_{\mathrm{n}} g_{\mathrm{b}} \\
g_{\mathrm{b}} g_{\mathrm{t}} & g_{\mathrm{b}} g_{\mathrm{n}} & g_{\mathrm{b}} g_{\mathrm{b}}
\end{array}\right) \\
& +\mathcal{F}_{2} \frac{1}{g_{*}}\left(\begin{array}{ccc}
g_{\mathrm{t}} & g_{\mathrm{n}} & g_{\mathrm{b}} \\
0 & 0 & 0 \\
0 & 0 & 0
\end{array}\right)
\end{aligned}
$$

Dabei wurden folgende Abkürzungen verwendet:

$$
\begin{aligned}
& \mathcal{F}_{1}=\frac{1}{\left(1-M_{\alpha}{ }^{2}\right)} \frac{\rho_{\mathrm{e}}}{p_{\mathrm{e}}} \frac{1}{\gamma} \frac{g_{*}}{\kappa} \\
& \mathcal{F}_{2}=-\mathcal{F}_{1}^{2}\left[(1-\nabla) M_{\alpha}{ }^{2}+\left(1-M_{\alpha}{ }^{2}\right) \nabla\right] \gamma \frac{g_{\mathrm{t}}}{g_{*}} \\
& \mathcal{F}_{3}=\mathcal{F}_{1}^{2} \frac{\gamma^{2}}{2} \Delta\left(1-M_{\alpha}{ }^{2}\right)
\end{aligned}
$$




$$
\Delta=\beta \delta-\frac{2}{\gamma}\left(\frac{1}{\gamma}-\frac{1}{2}\right)
$$

Die Komponenten $g_{\mathrm{x}}=\left(\mathbf{g}_{\text {eff }} \cdot \mathbf{x}\right), \mathbf{x} \in\left[\mathbf{t}_{0}, \mathbf{n}_{0}, \mathbf{b}_{0}\right]$ der effektiven Gravitationsbeschleunigung $\mathbf{g}_{\text {eff }}$ im Koordinatensystem (2.17) des Frenetschen Dreibeins können im toroidalen Gleichgewicht durch die Beziehungen (9.9) in Zylinderkoordinaten dargestellt werden.

Für toroidale Flussröhren in einem Einzelstern sind die Gleichgewichtsgrößen grundsätzlich konstant. In einem Doppelstern hingegen (Abschn. 9.2) zeigen die Gleichgewichtsgrößen entsprechend der Näherung (8.19) eine $\pi$-periodische Variation mit dem Azimutwinkel $\phi$; für die effektive Gravitationsbeschleunigung gelten etwa die Beziehungen (8.15) - (8.17). Setzt man diese Näherung in die linearisierte Bewegungsgleichung ein und vernachlässigt Terme der Ordnung $\epsilon^{4}$ und höher so erhält man nach dem $\mathrm{Zu}$ sammenfassen gleicher Ausdrücke für jede der Koeffizientmatrizen (A.3) - (A.6) die in Gl. (9.13) eingeführten Matrizen $\mathcal{M}_{\mathrm{c}}$ bzw. $\mathcal{M}_{\mathrm{s}}$.

Der Lösungsansatz (9.15) führt zur 3-Term-Rekursionsgleichung (9.16) bzw. zum Hillschen Gleichungssystem (9.17), in denen folgende Abkürzungen verwendet wurden:

$$
\begin{aligned}
\mathcal{C}_{n}= & -n^{2} \mathcal{E}-n \tilde{\omega} \mathcal{M}_{\phi t, 0}-\tilde{\omega}^{2} \mathcal{M}_{t t, 0}+\mathcal{M}_{\xi, 0}+i\left(n \mathcal{M}_{\phi, 0}+\tilde{\omega} \mathcal{M}_{t, 0}\right) \\
\mathcal{L}_{n}= & \frac{1}{2}\left[(n-2)\left(i \mathcal{M}_{\phi, c}+\mathcal{M}_{\phi, s}-\tilde{\omega}\left(\mathcal{M}_{\phi t, c}-i \mathcal{M}_{\phi t, s}\right)\right)\right. \\
& \left.+\mathcal{M}_{\xi, c}-i \mathcal{M}_{\xi, s}+\tilde{\omega}\left(i \mathcal{M}_{t, c}+\mathcal{M}_{t, s}\right)-\tilde{\omega}^{2}\left(\mathcal{M}_{t t, c}-i \mathcal{M}_{t t, s}\right)\right] \\
\mathcal{R}_{n}= & \frac{1}{2}\left[(n+2)\left(i \mathcal{M}_{\phi, c}-\mathcal{M}_{\phi, s}-\tilde{\omega}\left(\mathcal{M}_{\phi t, c}+i \mathcal{M}_{\phi t, s}\right)\right)\right. \\
& \left.+\mathcal{M}_{\xi, c}+i \mathcal{M}_{\xi, s}+\tilde{\omega}\left(i \mathcal{M}_{t, c}-\mathcal{M}_{t, s}\right)-\tilde{\omega}^{2}\left(\mathcal{M}_{t t, c}+i \mathcal{M}_{t t, s}\right)\right)
\end{aligned}
$$




\section{Literaturverzeichnis}

Abbett W.P., Fisher G.H., Fan Y., 2000, The three-dimensional evolution of rising, twisted magnetic flux tubes in a gravitationally stratified model convection zone, ApJ, 540, $548-562$

- 2001 , The effects of rotation on the evolution of rising Omega loops in a stratified model convection zone, ApJ, 546, 1194-1203

Abramowitz M., Stegun I.A., Handbook of mathematical functions (Dover, 1972)

Abt H.A., Levy S.G., 1976, Multiplicity among solar-type stars, ApJS, 30, 273-306

Alexander D.R., Ferguson J.W., 1994, Low-temperature rosseland opacities, ApJ, 437, 879-891

Antiochos S.K., Haisch B.M., Stern R.A., 1986, On the dividing line for stellar coronae, ApJL, 307, L55-L59

Ayres T.R., Fleming T.A., Schmitt J.H.M.M., 1991, Digging in the coronal graveyard - A ROSAT observation of the red giant Arcturus, ApJL, 376, L45-L48

Ayres T.R., Linsky J.L., Vaiana G.S., Golub L., Rosner R., 1981, The cool half of the H-R diagram in soft X-rays, ApJ, 250, 293-299

Ayres T.R., Simon T., Stern R.A., Drake S.A., Wood B.E., Brown A., 1998, The coronae of moderate-mass giants in the Hertzsprung gap and the clump, ApJ, 496, 428

Babcock H.W., 1961, The topology of the sun's magnetic field and the 22-year cycle, ApJ, 133, 572-587

Baliunas S.L., Vaughan A.H., 1985, Stellar activity cycles, Ann. Rev. Astron. Astroph., 23, 379-412

Basu S., 1997, Seismology of the base of the solar convection zone, Mon. Not. Royal Astron. Soc., 288, 572-584

Berdyugina S.V., Berdyugin A.V., Ilyin I., Tuominen I., 1999, The active RS Canum Venaticorum binary II Pegasi. IV. The spot activity cycle, A\&A, 350, 626-634

Berdyugina S.V., Tuominen I., 1998, Permanent active longitudes and activity cycles on RS CVn stars, A\&A, 336, L25-L28 
Bopp B.W., Fekel F., 1977, Binary incidence among the BY Draconis variables, Astron. J., 82, 490-494

Budding E., Zeilik M., 1987, An analysis of the light curves of short-period RS Canum Venaticorum stars - Starspots and fundamental properties, ApJ, 319, 827-835

Caligari P., 1991, Die Knick-Instabilität magnetischer Flußröhren in der solaren Konvektionszone, Diplomarbeit, Albert-Ludwigs-Universität, Freiburg/Brsg.

—, 1995, Dynamik magnetischer Flußröhren, Dissertation, Albert-Ludwigs-Universität, Freiburg/Brsg.

Caligari P., Moreno-Insertis F., Schüssler M., 1995, Emerging flux tubes in the solar convection zone. I. Asymmetry, tilt, and emergence latitude, ApJ, 441, 886-902

Caligari P., Schüssler M., Moreno-Insertis F., 1998, Emerging flux tubes in the solar convection zone. II. The influence of initial conditions, ApJ, 502, 481-492

Caughlan G.R., Fowler W.A., 1988, Thermonuclear reaction rates V, Atomic Data and Nuclear Data Tables, 40, 283

Charbonnel C., Talon S., 1999, The hot side of the lithium dip - LiBeB abundances beyond the main sequence, A\&A, 351, 635-643

Cheng J., 1992, Equations for the motion of an isolated thin magnetic flux tube, A\&A, 264, 243-248

Choudhuri A.R., 1989, The evolution of loop structures in flux rings within the solar convection zone, Sol. Phys., 123, 217-239

—, 1990, A correction to Spruit's equation for the dynamics of thin flux tubes, A\&A, 239, 335-339

Choudhuri A.R., Gilman P.A., 1987, The influence of the Coriolis force on flux tubes rising through the solar convection zone, ApJ, 316, 788-800

Claret A., Gimenez A., Physical processes in close binary systems, in: F.C. Lázaro, M.J. Arévalo (Hg.), Binary stars: Selected topics on observations and physical processes, Lecture Notes in Physics, vol. 563, S. 1 (2001)

Clayton D.D., Principles of stellar evolution and nucleosynthesis (New York: McGrawHill, 1968, 1968)

Collatz L., Differentialgleichungen (Teubner, 1970), 4. Aufl.

Collier Cameron A., Stellar prominences (review), in: IAU Symp. 176: Stellar surface structure, Bd. 176, S. 449 (1996)

—, 1997, Eclipse mapping of late-type close binary stars, Mon. Not. Royal Astron. Soc., 287, 556-566 
Cooper C.D., Alley F.C., Air Pollution - A design approach (Waveland Press, 1994), 2. Aufl.

Dempsey R.C., Linsky J.L., Fleming T.A., Schmitt J.H.M.M., 1997, The ROSAT All-Sky Survey of active binary coronae. III. Quiescent coronal properties for the BY Draconistype binaries, ApJ, 478, 358-366

Donahue R.A., 1993, Surface differential rotation in a sample of cool dwarf stars, Dissertation, New Mexica State University

Donati J.F., Brown S.F., Semel M., Rees D.E., Dempsey R.C., Matthews J.M., Henry G.W., Hall D.S., 1992, Photospheric imaging of the RS CVn system HR 1099, A\&A, 265, 682-700

Donati J.F., Semel M., Carter B.D., Rees D.E., Collier Cameron A., 1997, Spectropolarimetric observations of active stars, Mon. Not. Royal Astron. Soc., 291, 658-682

D'Silva S.Z., Choudhuri A.R., 1991, The effect of Kelvin-Helmholtz instability on rising flux tubes in the convection zone, Sol. Phys., 136, 201-219

Duncan D.K., Vaughan A.H., Wilson O.C., Preston G.W., Frazer J., Lanning H., Misch A., Mueller J., Soyumer D., Woodard L., Baliunas S.L., Noyes R.W., Hartmann L.W., Porter A., Zwaan C., Middelkoop F., Rutten R.G.M., Mihalas D., 1991, CA II H and K measurements made at Mount Wilson Observatory, 1966-1983, ApJS, 76, 383-430

Duquennoy A., Mayor M., 1991, Multiplicity among solar-type stars in the solar neighbourhood. II - Distribution of the orbital elements in an unbiased sample, A\&A, 248, $485-524$

Eaton J.A., Hall D.S., 1979, Starspots as the cause of the intrinsic light variations in RS Canum Venaticorum type stars, ApJ, 227, 907-922

Eaton J.A., Wu C.C., Rucinski S.M., 1980, Ultraviolet colors of W Ursae Majoris - Gravity darkening, temperature differences, and the cause of W-type light curves, ApJ, 239, 919-927

Emonet T., Moreno-Insertis F., 1998, The physics of twisted magnetic tubes rising in a stratified medium: Two-dimensional results, ApJ, 492, 804-821

Fan Y., Fisher G.H., Deluca E.E., 1993, The origin of morphological asymmetries in bipolar active regions, ApJ, 405, 390-401

Fan Y., Fisher G.H., McClymont A.N., 1994, Dynamics of emerging active region flux loops, ApJ, 436, 907-928

Fan Y., Zweibel E.G., Linton M.G., Fisher G.H., 1998, The rise of kink-unstable magnetic flux tubes in the solar convection zone, ApJL, 505, L59-L63

Fekel F.C., Moffett T.J., Henry G.W., 1986, A survey of chromospherically active stars, ApJS, 60, 551-576 
Ferriz-Mas A., 1996, On the storage of magnetic flux tubes at the base of the solar convection zone, ApJ, 458, 802-816

Ferriz-Mas A., Schmitt D., Schüssler M., 1994, A dynamo effect due to instability of magnetic flux tubes, A\&A, 289, 949-956

Ferriz-Mas A., Schüssler M., 1993, Instabilities of magnetic flux tubes in a stellar convection zone. I. Equatorial flux rings in differentially rotating stars, Geophys. Astrophys. Fluid Dyn., 72, 209-247

—, 1995, Instabilities of magnetic flux tubes in a stellar convection zone. II. Flux rings outside the equatorial plane, Geophys. Astrophys. Fluid Dyn., 81, 233-265

Ferriz-Mas A., Schüssler M., Anton V., 1989, Dynamics of magnetic flux concentrations - The second-order thin flux tube approximation, A\&A, 210, 425-432

Fischer A., Beuermann K., 2001, Accretion physics of AM Herculis binaries. I. Results from one-dimensional stationary radiation hydrodynamics, A\&A, 373, 211-221

Gaensicke B.T., Beuermann K., de Martino D., 1995, The white dwarf in AM Herculis., A\&A, 303, 127-136

Galloway D.J., Weiss N.O., 1981, Convection and magnetic fields in stars, ApJ, 243, 945-953

Gautschy A., 2001, Zustandsgleichung, priv. comm.

Gershberg R.E., Katsova M.M., Lovkaya M.N., Terebizh A.V., Shakhovskaya N.I., 1999, Catalogue and bibliography of the UV Cet-type flare stars and related objects in the solar vicinity, A\&AS, 139, 555-558

Granzer T., 2000, Dünne magnetische Flußröhren in jungen, schnell rotierenden Sternen, Dissertation, Universität Wien

Granzer T., Schüssler M., Caligari P., Strassmeier K.G., 2000, Distribution of starspots on cool stars. II. Pre-main-sequence and ZAMS stars between $0.4 M_{\odot}$ and $1.7 M_{\odot}$, A\&A, 355, 1087-1097

Gray D.F., 1989, The rotational break for G giants, ApJ, 347, 1021-1029

—, Rotation of evolved stars, in: S. Catalano, J.R. Stauffer (Hg.), Angular Momentum Evolution of Young Stars, S. 183 (Kluwer, Dordrecht, 1991)

Gray D.F., Nagel T., 1989, The granulation boundary in the H-R diagram, ApJ, 341, 421426

Haisch B., Schmitt J.H.M.M., Fabian A.C., 1992a, Disappearance of coronal X-ray emission in stars with cool dense winds, Nature, 360, 239-241

Haisch B., Schmitt J.H.M.M., Rosso C., 1991, The coronal dividing line in the ROSAT X-ray All-Sky Survey, ApJL, 383, L15-L18 
—, 1992b, ROSAT X-ray All-Sky Survey observations of hybrid stars, ApJL, 388, L61L64

Haisch B.M., Bookbinder J.A., Maggio A., Vaiana G.S., Bennett J.O., 1990, IUE and Einstein survey of late-type giant and supergiant stars and the dividing line, ApJ, 361, $570-589$

Hall D.S., The RS CVn binaries and binaries with similar properties, in: ASSL Vol. 60: IAU Colloq. 29: Multiple Periodic Variable Stars, S. 287 (1976)

- Learning about stellar dynamos from longterm photometry of starspots, in: I. Tuominen, D. Moss, G. Rüdiger (Hg.), The Sun and Cool Stars. Activity, Magnetism, Dynamos, S. 353 (1991)

Heckert P.A., Maloney G.V., Stewart M.C., Ordway J.I., Hickman A., Zeilik M., 1998, A Decade of starspot activity on the eclipsing short-period RS Canum Venaticorum star WY Cancri: 1988-1997, Astron. J., 115, 1145-1152

Heckert P.A., Ordway J.I., 1995, Long term spot activity on eclipsing RS CVn stars: RS CVn, SS Boo, and MM Her, Astron. J., 109, 2169-2176

Henry G.W., Eaton J.A., Hamer J., Hall D.S., 1995, Starspot evolution, differential rotation, and magnetic cycles in the chromospherically active binaries $\lambda$ Andromedae, $\sigma$ Geminorum, II Pegasi, and V711 Tauri, ApJS, 97, 513-549

Holzwarth V., Schüssler M., 2001, Buried magnetic flux tubes in giant stars near the "Coronal Dividing Line", A\&A, 377, 251-263

Holzwarth V., Schüssler M., 2000, Stability of magnetic flux tubes in close binary stars, Astronomische Nachrichten, 321, 175-179

Hünsch M., Schmitt J.H.M.M., Schröder K., Reimers D., 1996, ROSAT X-ray observations of a complete, volume-limited sample of late-type giants, A\&A, 310, 801-812

Hünsch M., Schmitt J.H.M.M., Schröder K., Zickgraf F., 1998, On the X-ray emission from M-type giants, A\&A, 330, 225-231

Hünsch M., Schröder K., 1996, The revised X-ray dividing line: New light on late stellar activity, A\&A, 309, L51-L54

Hut P., 1980, Stability of tidal equilibrium, A\&A, 92, 167-170

—, 1981, Tidal evolution in close binary systems, A\&A, 99, 126-140

Iglesias C.A., Rogers F.J., 1996, Updated OPAL opacities, ApJ, 464, 943-953

Jardine M., Collier Cameron A., Donati J.F., Pointer G.R., 2001, Prominence support in potential field configurations around rotating stars, Mon. Not. Royal Astron. Soc., 324, 201-205 
Jetsu L., 1996, The active longitudes of $\lambda$ Andromedae, $\sigma$ Geminorum, II Pegasi and V 711 Tauri., A\&A, 314, 153-164

Kashyap V., Rosner R., Harnden F.R., Maggio A., Micela G., Sciortino S., 1994, X-ray emission on hybird stars: ROSAT observations of $\alpha$ Trianguli Australis and $\iota$ Aurigae, ApJ, 431, 402-415

Kippenhahn R., Möllenhoff C., Elementare Plasmaphysik (Bibliogr. Inst., 1975)

Kippenhahn R., Weigert A., Hofmeister E., 1967, Methods for calculating stellar evolution, Meth. Comp. Phys., 7, 129-190

Kopal Z., Close binary systems (Chapman \& Hall, 1959)

Lanza A.F., Catalano S., Cutispoto G., Pagano I., Rodonò M., 1998, Long-term starspot evolution, activity cycle and orbital period variation of AR Lacertae, A\&A, 332, 541560

Lanza A.F., Catalano S., Rodonò M., İbanoğlu C., Evren S., Taş G., Çakırlı Ö., Devlen A., 2002, Long-term starspot evolution, activity cycle and orbital period variation of RT Lacertae, A\&A, 386, 583-605

Lanza A.F., Rodonò M., Mazzola L., Messina S., 2001, Long-term starspot evolution, activity cycle and orbital period variation of SZ Piscium, A\&A, 376, 1011-1030

Leighton R.B., 1964, Transport of magnetic fields on the sun, ApJ, 140, 1547-1562

—, 1969, A magneto-kinematic model of the solar cycle, ApJ, 156, 1-26

Linsky J.L., Haisch B.M., 1979, Outer atmospheres of cool stars. I. The sharp division into solar-type and non-solar-type stars, ApJL, 229, L27-L30

Longcope D.W., Fisher G.H., Arendt S., 1996, The evolution and fragmentation of rising magnetic flux tubes, ApJ, 464, 999-1011

Maggio A., Vaiana G.S., Haisch B.M., Stern R.A., Bookbinder J., Harnden F.R., Rosner R., 1990, Einstein Observatory magnitude-limited X-ray survey of late-type giant and supergiant stars, ApJ, 348, 253-278

Moreno-Insertis F., 1984, Die dynamische Entwicklung von magnetischen Flußröhren in der Konvektionszone der Sonne, Dissertation, Ludwig-Maximilians-Universität, München

— 1986, Nonlinear time-evolution of kink-unstable magnetic flux tubes in the convective zone of the sun, A\&A, 166, 291-305

Moreno-Insertis F., Ferriz-Mas A., Schüssler M., 1994, Forces on magnetic flux tubes moving in inhomogeneous flows, ApJ, 422, 652-658

Moreno-Insertis F., Schüssler M., Ferriz-Mas A., 1992, Storage of magnetic flux tubes in a convective overshoot region, A\&A, 264, 686-700 
—, 1996, Enhanced inertia of thin magnetic flux tubes, A\&A, 312, 317-326

Morse P.M., Feshbach H., Methods of theoretical physics (McGraw-Hill, 1953)

Narain U., Ulmschneider P., 1990, Chromospheric and coronal heating mechanisms, Space Science Reviews, 54, 377-445

—, 1996, Chromospheric and Coronal Heating Mechanisms II, Space Science Reviews, 75, 453-509

Olah K., Budding E., Kim H.L., Etzel P.B., 1994, The active close binary system ER Vulpeculae, A\&A, 291, 110-120

O’Neal D., Saar S.M., Neff J.E., 1998, Spectroscopic evidence for nonuniform starspot properties on II Pegasi, ApJL, 501, L73-L76

Ossendrijver M., Hoyng P., Solar cycle, in: Encyclopedia of Astronomy and Astrophysics (Nature Publishing Group, 2001)

Osten R.A., Brown A., 1999, Extreme Ultraviolet Explorer photometry of RS Canum Venaticorum systems: Four flaring Megaseconds, ApJ, 515, 746-761

Osten R.A., Brown A., Ayres T.R., Linsky J.L., Drake S.A., Gagné M., Stern R.A., 2000, Radio, X-Ray, and Extreme-Ultraviolet coronal variability of the short-period RS Canum Venaticorum binary $\sigma^{2}$ Coronae Borealis, ApJ, 544, 953-976

Parker E.N., 1955a, Hydromagnetic dynamo models, ApJ, 122, 293-314

$\ldots, 1955 \mathrm{~b}$, The formation of sunspots from the solar toroidal field, ApJ, 121, 491-507

—, 1966, The dynamical state of the interstellar gas and field, ApJ, 145, 811-833

_, 1993, A solar dynamo surface wave at the interface between convection and nonuniform rotation, ApJ, 408, 707-719

Pidatella R.M., Stix M., 1986, Convective overshoot at the base of the sun's convection zone, A\&A, 157, 338-340

Pintér B., C̆adež V.M., Roberts B., 1999, Waves and instabilities in a stratified isothermal atmosphere with constant Alfvén speed - revisited, A\&A, 346, 190-198

Priest E.R., Solar Magneto-Hydrodynamics, Geophysics and Astrophysics Monographs (D. Reidel, 1982)

Reimers D., 1977, Observational evidence for mass-loss from K giants, G and K supergiants, A\&A, 57, 395-400

Reimers D., Hünsch M., Schmitt J.H.M.M., Toussaint F., 1996, Hybrid stars and the reality of 'dividing lines' among $G$ to $K$ bright giants and supergiants, A\&A, 310, 813-824 
Reimers D., Schmitt J.H.M.M., 1992, ROSAT X-ray observations of hybrid stars, ApJL, 392, L55-L58

Roberts B., Webb A.R., 1978, Vertical motions in an intense magnetic flux tube, Sol. Phys., 56, 5-35

Rodonò M., Messina S., Lanza A.F., Cutispoto G., Teriaca L., 2000, The magnetic activity cycle of II Pegasi: Results from twenty-five years of wide-band photometry, A\&A, 358, $624-638$

Rodonò M., Cutispoto G., Pazzani V., Catalano S., Byrne P.B., Doyle J.G., Butler C.J., Andrews A.D., Blanco C., Marilli E., Linsky J.L., Scaltriti F., Busso M., Cellino A., Hopkins J.L., Okazaki A., Hayashi S.S., Zeilik M., Helston R., Henson G., Smith P., Simon T., 1986, Rotational modulation and flares on RS CVn and BY Dra-type stars. I - Photometry and spot models for BY Dra, AU Mic, AR Lac, II Peg and V 711 Tau (= HR 1099), A\&A, 165, 135-156

Rodonò M., Lanza A.F., Catalano S., 1995, Starspot evolution, activity cycle and orbital period variation of the prototype active binary RS Canum Venaticorum., A\&A, 301, $75-88$

Rogers F.J., Swenson F.J., Iglesias C.A., 1996, OPAL equation-of-state tables for astrophysical applications, ApJ, 456, 902-908

Rosner R., An C.., Musielak Z.E., Moore R.L., Suess S.T., 1991, Magnetic confinement, Alfven wave reflection, and the origins of $\mathrm{X}$-ray and mass-loss 'dividing lines' for latetype giants and supergiants, ApJL, 372, L91-L94

Rosner R., Musielak Z.E., Cattaneo F., Moore R.L., Suess S.T., 1995, On the origin of 'dividing lines' for late-type giants and supergiants, ApJL, 442, L25-L28

Rüdiger G., von Rekowski B., Donahue R.A., Baliunas S.L., 1998, Differential rotation and meridional flow for fast-rotating solar-type stars, ApJ, 494, 691-699

Schmidt-Kaler T., Astronomy and Astrophysics, Bd. 2 von Landolt-Börnstein - New Series, Grp.VI, Kap. 4.1 Physical Parameters of the Stars, S. 456 (Springer, 1982)

Schmitt D., 1998, On the stability of stationary flows in thin magnetic flux tubes, Geophys. Astrophy. Fluid Dyn., 89, 75-143

Schmitt J.H.M.M., 1997, Coronae on solar-like stars., A\&A, 318, 215-230

Schrijver C.J., Title A.M., 2001, On the formation of polar spots in sun-like stars, ApJ, 551, 1099-1106

Schrijver C.J., Zwaan C., Solar and Stellar Magnetic Activity, Cambride Astrophysics Series (Cambridge University Press, 2000)

Schüssler M., 1979, Magnetic buoyancy revisited - Analytical and numerical results for rising flux tubes, A\&A, 71, 79-91 
Schüssler M., Caligari P., Ferriz-Mas A., Moreno-Insertis F., 1994, Instability and eruption of magnetic flux tubes in the solar convection zone, A\&A, 281, L69-L72

Schüssler M., Caligari P., Ferriz-Mas A., Solanki S.K., Stix M., 1996, Distribution of starspots on cool stars. I. Young and main sequence stars of $1 M_{\odot}$, A\&A, 314, 503-512

Schüssler M., Holzwarth V., Charbonnel C., Solanki S.K., Buried flux tubes in the coronal graveyard, in: R.J. García López, R. Rebolo, M.R. Zapatero Osorio (Hg.), $11^{\text {th }}$ Cambridge Workshop on Cool Stars, Stellar Systems and the Sun, Bd. 223 von ASP Conf. Series, S. CD-1114 (2001)

Schüssler M., Solanki S.K., 1992, Why rapid rotators have polar spots, A\&A, 264, L13L16

Semel M., 1989, Zeeman-Doppler imaging of active stars. I - Basic principles, A\&A, 225, 456-466

Shaviv G., Salpeter E.E., 1973, Convective overshooting in stellar interior models, ApJ, 184, 191-200

Skaley D., Stix M., 1991, The overshoot layer at the base of the solar convection zone, A\&A, 241, 227-232

Spitzer L., Physics of fully ionized gases (John Wiley \& Sons, 1962)

Spruit H.C., 1981, Equations for thin flux tubes in ideal MHD, A\&A, 102, 129-133

Spruit H.C., van Ballegooijen A.A., 1982, Stability of toroidal flux tubes in stars, A\&A, 106, 58-66

Stenflo J.O., 1989, Small-scale magnetic structures on the sun, A\&AR, 1, 3-48

Stix M., The Sun - An Introduction, Astronomy \& Astrophysics Library (Springer Verlag, 1989)

Strassmeier K.G., 1994, Rotational-modulation mapping of the active atmosphere of the RS Canum Venaticorum binary HD 106225, A\&A, 281, 395-420

—, 1999, Doppler imaging of stellar surface structure. XI. The super starspots on the K0 giant HD 12545: larger than the entire Sun, A\&A, 347, 225-234

Strassmeier K.G., Bartus J., 2000, Doppler imaging of stellar surface structure. XII. Rapid spot changes on the RS CVn binary V711 Tauri $=$ HR 1099, A\&A, 354, 537-550

Strassmeier K.G., Hall D.S., Fekel F.C., Scheck M., 1993, A catalog of chromospherically active binary stars (second edition), A\&AS, 100, 173-225

Tassoul J., 1995, On orbital circulation in late-type close binaries, ApJ, 444, 338-341

Tassoul J., Tassoul M., 1992, A comparative study of synchronization and circularization in close binaries, ApJ, 395, 259-267 
Unsöld A., Baschek B., Der neue Kosmos (Springer Verlag, 1991)

van Ballegooijen A.A., 1982, The overshoot layer at the base of the solar convective zone and the problem of magnetic flux storage, A\&A, 113, 99-112

Vogt S.S., Penrod G.D., 1983, Doppler Imaging of spotted stars - Application to the RS Canum Venaticorum star HR 1099, Publ. Astron. Soc. Pac., 95, 565-576

Vogt S.S., Penrod G.D., Hatzes A.P., 1987, Doppler images of rotating stars using maximum entropy image reconstruction, ApJ, 321, 496-515

Walter W., Gewöhnliche Differentialgleichungen (Springer Verlag, 1996), 6. Aufl.

Weiss N., Sunspots, in: Encyclopedia of Astronomy and Astrophysics (Nature Publishing Group, 2001)

Wilson O.C., 1968, Flux measurements at the centers of stellar $\mathrm{H}$ - and K-lines, ApJ, 153, 221-234

Yakubovich V.A., Starzhinskii V.M., Linear differential equations with periodic coefficients (John Wiley \& Sons, 1975)

Zahn J.P., 1977, Tidal friction in close binary stars, A\&A, 57, 383-394

—, 1991, Convective penetration in stellar interiors, A\&A, 252, 179-188

Zeilik M., Magnetic Activity of Short-Period RS CVN Stars, in: ASP Conf. Ser. 26: Cool Stars, Stellar Systems, and the Sun, Bd. 7, S. 346+ (1992)

Zeilik M., Cox D.A., de Blasi C., Rhodes M., Budding E., 1989, Long-term starspot activity of short-period RS Canum Venaticorum stars. II - RT Andromedae, ApJ, 345, 991-997

Zeilik M., Cox D.A., Ledlow M.J., Rhodes M., Heckert P.A., Budding E., 1990a, Longterm starspot activity of short-period RS Canum Venaticorum stars. IV - WY Cancri, ApJ, 363, 647-653

Zeilik M., Gordon S., Jaderlund E., Ledlow M., Summers D.L., Heckert P.A., Budding E., Banks T.S., 1994, Long-term starspot activity of short-period RS Canum Venaticorum stars. V - CG Cygni, ApJ, 421, 303-309

Zeilik M., Ledlow M., Rhodes M., Arevalo M.J., Budding E., 1990b, Long-term starspot activity of short-period RS Canum Venaticorum stars. III - BH Virginis, ApJ, 354, 352358

Zwaan C., 1978, On the appearance of magnetic flux in the solar photosphere, Sol. Phys., 60, 213-240 


\section{Danksagung}

Die vorliegende Arbeit wurde am Kiepenheuer-Institut für Sonnenphysik in Freiburg/Brsg. und am Max-Planck-Institut für Aeronomie in Katlenburg-Lindau angefertigt. Für die angenehme Arbeitsatmosphäre und vielfältige Unterstützung in beiden Instituten möchte ich mich bei allen Mitarbeitern und Kollegen herzlich bedanken.

Den Direktoren Herrn Prof. O. von der Lühe und Herrn Prof. M. Stix sowie Herrn Prof. S. K. Solanki danke ich für die Möglichkeit zur Durchführung dieser Arbeit, die Gewährung finanzieller Unterstützung und die Gelegenheit zur Teilnahme an verschiedenen Tagungen und Sommerschulen.

Mein Dank gilt besonders Herrn Prof. M. Schüssler für die Vergabe des Themas, die ausgezeichnete Betreuung der Arbeit und die kritischen Diskussionen — nicht nur — über die Ergebnisse. Herrn Prof. K. Beuermann danke ich für die Übernahme der Betreuung seitens der Universität Göttingen.

Bedanken möchte ich mich auch bei meinen langjährigen 'Mitstreitern' Herrn Dr. M. Rempel und Herrn Dr. P. Vollmöller für das sehr lebhafte, abwechslungsreiche, informative $^{1}$ und bisweilen auch esoterische Arbeitsklima. Bei Herrn A. Vögler sowie allen weiteren derzeitigen und ehemaligen Mitgliedern der Kaffeerunden bedanke ich mich für die interessanten Gespräche. Spezieller Dank gebührt dabei meinem Kollegen und 'Zellennachbar' Herrn O. Preuss für seine ständige Gesprächs-, Hilfs- und Fahrbereitschaft.

Herrn Dr. P. Caligari und Herrn Dr. T. Granzer danke ich für die Bereitstellung verschiedener Computerprogramme und ihrer Unterstützung bei fachlichen, numerischen und diversen EDV-Problemen. Frau Dr. C. Charbonnel danke ich für die Bereitstellung verschiedener Sternmodelle und -entwicklungssequenzen.

Herrn Dr. J. Bruls und Herrn Dr. D. Schmitt danke ich für zahlreiche informative und unterhaltsame Gesprächsrunden und ihre kompetente Hilfe bei Problemen z.B. mit IDL oder TeX. Herrn Prof. F. Moreno-Insertis danke ich für seine fachliche Unterstützung und Beratung, insbesondere während meines Besuchs am IAC auf Teneriffa.

Mein größter Dank gebührt allerdings meinen Eltern und meinem Bruder für ihre ausdauernde Unterstützung, ihren Rat und ihre Anteilnahme an meiner Arbeit.

\footnotetext{
${ }^{1}$ Die fundamentale Frage nach der bürgerlichen Klassifizierung der Culex pipiens ist m.E. noch nicht abschließend geklärt.
} 


\section{Lebenslauf}

5. Januar 1971 geboren in Ludwigshafen am Rhein

Eltern Karl Holzwarth, Betriebsleiter (i.R.)

Marlise Holzwarth, geb. Keck, Hausfrau

Staatsbürgerschaft deutsch

Schulausbildung

9/1977-6/1981 Johann-Hoffmann-Grundschule, Mutterstadt

8/1981-6/1990 Carl-Bosch-Gymnasium, Ludwigshafen/Rhein

6/1990 Allgemeine Hochschulreife

Wehrdienst

7/1990-6/1991 Grundwehrdienst, Weingarten, Bad Bergzabern

Studium

10/1991-10/1997 Physikstudium an der Ruprecht-Karls-Universität Heidelberg

2/1994 Vordiplomprüfung

9/1996-10/1997 Diplomarbeit, Institut für Theoretische Astrophysik, Heidelberg

Thema: Die Ausbreitung und Kopplung longitudinaler und torsionaler Wellen in dünnen magnetischen Flußröhren

10/1997 Diplomprüung

seit 11/99 Studium an der Georg-August-Universität, Göttingen

Anstellungen

11/1997-10/99 Kiepenheuer-Institut für Sonnenphysik, Freiburg/Brsg. wissenschaftlicher Angestellter (Doktorand)

seit 11/99 Max-Planck-Institut für Aeronomie, Katlenburg-Lindau, Max-Planck-Stipendiat 